\title{
THE BOUNDED AND PRECISE WORD PROBLEMS FOR PRESENTATIONS OF GROUPS
}

\author{
S. V. IVANOV
}

\begin{abstract}
We introduce and study the bounded word problem and the precise word problem for groups given by means of generators and defining relations. For example, for every finitely presented group, the bounded word problem is in NP, i.e., it can be solved in nondeterministic polynomial time, and the precise word problem is in PSPACE, i.e., it can be solved in polynomial space. The main technical result of the paper states that, for certain finite presentations of groups, which include the Baumslag-Solitar one-relator groups and free products of cyclic groups, the bounded word problem and the precise word problem can be solved in polylogarithmic space. As consequences of developed techniques that can be described as calculus of brackets, we obtain polylogarithmic space bounds for the computational complexity of the diagram problem for free groups, for the width problem for elements of free groups, and for computation of the area defined by polygonal singular closed curves in the plane. We also obtain polynomial time bounds for these problems.
\end{abstract}

\section{Contents}

1. Introduction

2. Preliminaries

3. Proof of Proposition 1.1

4. Calculus of Brackets for Group Presentation (1.2) 13

5. Proofs of Theorem 1.2 and Corollary $1.3 \quad 34$

6. Calculus of Brackets for Group Presentation (1.4) 38

7. Proof of Theorem 1.4

8. Minimizing Diagrams over (1.2) and Proofs of Theorem 1.5 and Corollary 1.6

9. Construction of Minimal Diagrams over (1.4) and Proof of Theorem 1.772

10. Polygonal Curves in the Plane and Proofs of Theorems 1.8, 1.9 and Corollary 1.10

References

2010 Mathematics Subject Classification. Primary 20F05, 20F06, 20F10, 68Q25, 68U05; Secondary 52B05, 20F65, 68W30.

Key words and phrases. Presentations of groups, diagrams, word problems, polylogarithmic space, polygonal curves.

The work on this article was supported in smaller part by NSF grant DMS 09-01782. 


\section{INTRODUCTION}

Suppose that a finitely generated group $\mathcal{G}$ is defined by a presentation by means of generators and defining relations

$$
\mathcal{G}=\langle\mathcal{A} \| R=1, R \in \mathcal{R}\rangle
$$

where $\mathcal{A}=\left\{a_{1}, \ldots, a_{m}\right\}$ is a finite alphabet and $\mathcal{R}$ is a set of defining relators which are nonempty cyclically reduced words over the alphabet $\mathcal{A}^{ \pm 1}:=\mathcal{A} \cup \mathcal{A}^{-1}$. Let $\mathcal{F}(\mathcal{A})$ denote the free group over $\mathcal{A}$, let $|W|$ be the length of a word $W$ over the alphabet $\mathcal{A}^{ \pm 1}$, and let $\mathcal{N}(\mathcal{R})$ denote the normal closure of $\mathcal{R}$ in $\mathcal{F}(\mathcal{A})$. The notation (1.1) implies that $\mathcal{G}$ is the quotient group $\mathcal{F}(\mathcal{A}) / \mathcal{N}(\mathcal{R})$. We will say that a word $W$ over $\mathcal{A}^{ \pm 1}$ is equal to 1 in the group $\mathcal{G}$, given by (1.1), if $W \in \mathcal{N}(\mathcal{R})$ in which case we also write $W \stackrel{\mathcal{G}}{=} 1$. Recall that the presentation (1.1) is called finite if both the sets $\mathcal{A}$ and $\mathcal{R}$ are finite, in which case $\mathcal{G}$ is called finitely presented. The presentation (1.1) is called decidable if there is an algorithm that decides whether a given word over $\mathcal{A}^{ \pm 1}$ belongs to $\mathcal{R}$.

The classical word problem for a finite group presentation (1.1), put forward by Dehn [10] in 1911, asks whether, for a given word $W$ over $\mathcal{A}^{ \pm 1}$, it is true that $W \stackrel{\underline{G}}{=} 1$. The word problem is said to be solvable (or decidable) for a decidable presentation (1.1) if there exists an algorithm which, given a word $W$ over $\mathcal{A}^{ \pm 1}$, decides whether or not $W \stackrel{\underline{G}}{=} 1$.

Analogously to Anisimov [1, one might consider the set of all words $W$ over

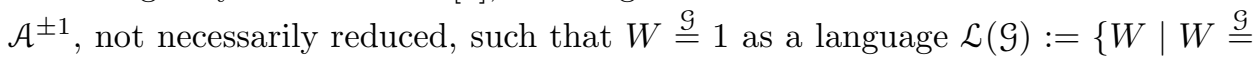
1 ) over $\mathcal{A}^{ \pm 1}$ and inquire about a computational complexity class $\mathrm{K}$ which would contain this language. If $\mathcal{L}(\mathcal{G})$ is in $\mathrm{K}$, we say that the word problem for $\mathcal{G}$ is in K. For example, it is well known that the word problem is in P, i.e., solvable in deterministic polynomial time, for surface groups, for finitely presented groups with small cancellation condition $C^{\prime}(\lambda)$, where $\lambda \leq \frac{1}{6}$, for word hyperbolic groups, for finitely presented groups given by Dehn presentations etc., see [13, [18. On the other hand, according to results of Novikov [25], 26] and Boone [6], [7, based on earlier semigroup constructions of Turing 39] and Post [34, see also Markov's papers [22, 23, there exists a finitely presented group $\mathcal{G}$ for which the word problem is unsolvable, i.e., there is no algorithm that decides whether $W \stackrel{\mathcal{G}}{=} 1$. The proof of this remarkable Novikov-Boone theorem was later significantly simplified by Borisov [8], see also [37.

In this paper, we introduce and study two related problems which we call the bounded word problem and the precise word problem for a decidable group presentation (1.1).

The bounded word problem for a decidable presentation (1.1) inquires whether, for given a word $W$ over $\mathcal{A}^{ \pm 1}$ and an integer $n \geq 0$ written in unary, denoted $1^{n}$, one can represent the word $W$ as a product in $\mathcal{F}(\mathcal{A})$ of at most $n$ conjugates of some words in $\mathcal{R}^{ \pm 1}:=\mathcal{R} \cup \mathcal{R}^{-1}$, i.e., whether there are $R_{1}, \ldots, R_{k} \in \mathcal{R}^{ \pm 1}$ and $S_{1}, \ldots, S_{k} \in \mathcal{F}(\mathcal{A})$ such that $W=S_{1} R_{1} S_{1}^{-1} \ldots S_{k} R_{k} S_{k}^{-1}$ in $\mathcal{F}(\mathcal{A})$ and $k \leq n$. Equivalently, for given an input $\left(W, 1^{n}\right)$, the bounded word problem asks whether there exists a disk diagram over (1.1), also called a van Kampen diagram, whose boundary label is $W$ and whose number of faces is at most $n$.

As above for the word problem, we say that the bounded word problem for a decidable presentation (1.1) is solvable if there exists an algorithm that, given an input 
$\left(W, 1^{n}\right)$, decides whether $W$ is a product in $\mathcal{F}(\mathcal{A})$ of at most $n$ conjugates of words in $\mathcal{R}^{ \pm 1}$. Analogously, if the language of those pairs $\left(W, 1^{n}\right)$, for which the bounded word problem has a positive answer, belongs to a computational complexity class $\mathrm{K}$, then we say that the bounded word problem for $\mathcal{G}$ is in $\mathrm{K}$.

The precise word problem for a decidable presentation (1.1) asks whether, for given a word $W$ over $\mathcal{A}^{ \pm 1}$ and a nonnegative integer $1^{n}$, one can represent the word $W$ as a product in $\mathcal{F}(\mathcal{A})$ of $n$ conjugates of words in $\mathcal{R}^{ \pm 1}$ and $n$ is minimal with this property. Equivalently, for given an input $\left(W, 1^{n}\right)$, the precise word problem asks whether there exists a disk diagram over (1.1) whose boundary label is $W$, whose number of faces is $n$, and there are no such diagrams with fewer number of faces.

The definitions for the precise word problem for (1.1) being solvable and being in a complexity class $\mathrm{K}$ are similar to the corresponding definitions for the (bounded) word problem.

In the following proposition we list basic comparative properties of the standard word problem and its bounded and precise versions.

Proposition 1.1. (a) There exists a decidable group presentation (1.1) for which the word problem is solvable while the bounded and precise word problems are not solvable.

(b) If the bounded word problem is solvable for (1.1), then the precise word problem is also solvable.

(c) For every finite group presentation (1.1), the bounded word problem is in $N P$, i.e., it can be solved in nondeterministic polynomial time, and the precise word problem is in PSPACE, i.e., it can be solved in polynomial space.

(d) There exists a finite group presentation (1.1) for which the bounded and precise word problems are solvable while the word problem is not solvable.

(e) There exists a finitely presented group (1.1) for which the bounded word problem is NP-complete and the precise word problem is NP-hard.

Note that a number of interesting results on solvability of the word problem, solvability of the bounded word problem and computability of the Dehn function for decidable group presentations can be found in a preprint of Cummins [9] based on his $\mathrm{PhD}$ thesis written under the author's supervision.

It is of interest to look at the bounded and precise word problems for finitely presented groups for which the word problem could be solved very easily. Curiously, even for very simple presentations such as $\langle a, b \| a=1\rangle$ and $\langle a, b \| a b=b a\rangle$, for which the word problem is obviously in $\mathrm{L}$, i.e., solvable in deterministic logarithmic space, it does not seem to be possible to solve the bounded and precise word problem in logarithmic space. In this article, we will show that the bounded and precise word problems for these "simple" presentations and their generalizations can be solved in polynomial time. With much more effort, we will also prove that the bounded and precise word problems for such presentations can be solved in polylogarithmic space. Similarly to [2, we adopt NC-style notation and denote $\mathrm{L}^{\alpha}:=\operatorname{DSPACE}\left((\log s)^{\alpha}\right)$, i.e., $\mathrm{L}^{\alpha}$ is the class of decision problems that can be solved in deterministic space $O\left((\log s)^{\alpha}\right)$, where $s$ is the size of input.

Theorem 1.2. Let the group $\mathcal{G}_{2}$ be defined by a presentation of the form

$$
\mathcal{G}_{2}:=\left\langle a_{1}, \ldots, a_{m} \| a_{i}^{k_{i}}=1, k_{i} \in E_{i}, i=1, \ldots, m\right\rangle,
$$

where for every $i$, one of the following holds: $E_{i}=\{0\}$ or, for some integer $n_{i}>0$, $E_{i}=\left\{n_{i}\right\}$ or $E_{i}=n_{i} \mathbb{N}=\left\{n_{i}, 2 n_{i}, 3 n_{i}, \ldots\right\}$. Then both the bounded and precise 
word problems for (1.2) are in $L^{3}$ and in $P$. Specifically, the problems can be solved in deterministic space $O\left((\log |W|)^{3}\right)$ or in deterministic time $O\left(|W|^{4} \log |W|\right)$.

It is worth mentioning that, to prove $\mathrm{L}^{3}$ part of Theorem 1.2, we will not devise a concrete algorithm that solves the bounded and precise word problems for presentation (1.2) in deterministic space $O\left((\log |W|)^{3}\right)$. Instead, we will develop a certain nondeterministic procedure that solves the bounded word problem for presentation (1.2) nondeterministically in space $O\left((\log |W|)^{2}\right)$ and time $O(|W|)$ and then use Savitch's theorem [38] on conversion of nondeterministic computations in space $S$ and time $T$ into deterministic computations in space $O(S \log T)$.

The proof of $\mathrm{P}$ part of Theorem 1.2 is much easier. Here our arguments are analogous to folklore arguments [35], 36] that solve the precise word problem for presentation $\langle a, b \| a=1, b=1\rangle$ in polynomial time and that utilize the method of dynamic programming. Interestingly, these folklore arguments are strikingly similar to the arguments that have been used in computational biology to efficiently solve the problem of planar folding of long chains such as RNA and DNA biomolecules, see [27], 28], 29], [40].

The techniques to prove $\mathrm{L}^{3}$ part of Theorem 1.2 and their generalizations, that occupy a significant part of this article and that could be described as calculus of bracket systems, have applications to other problems. For example, Grigorchuk and Kurchanov [11] defined the width of an element $W$ of the free group $\mathcal{F}(\mathcal{A})$ over $\mathcal{A}$ as the minimal number $h=h(W)$ so that

$$
W=S_{1} a_{j_{1}}^{k_{1}} S_{1}^{-1} \ldots S_{h} a_{j_{h}}^{k_{h}} S_{h}^{-1}
$$

in $\mathcal{F}(\mathcal{A})$, where $a_{j_{1}}, \ldots, a_{j_{h}} \in \mathcal{A}, S_{1}, \ldots, S_{h} \in \mathcal{F}(\mathcal{A})$ and $k_{1}, \ldots, k_{h}$ are some integers. Alternatively, the width $h(W)$ of $W$ can be defined as an integer such that the precise word problem holds for the pair $(W, h(W))$ for the presentation (1.2) in which $E_{i}=\mathbb{N}$ for every $i$. Grigorchuk and Kurchanov [11] found an algorithm that computes the width $h(W)$ for given $W \in \mathcal{F}(\mathcal{A})$ and inquired whether computation of the width $h(W)$ can be done in deterministic polynomial time. Ol'shanskii 31] gave a different geometric proof to this result of Grigorchuk and Kurchanov and suggested some generalizations.

Majumdar, Robbins, and Zyskin [20, 21] introduced and investigated the spelling length $h_{1}(W)$ of a word $W \in \mathcal{F}(\mathcal{A})$ defined by a similar to (1.3) formula in which $k_{j}= \pm 1$ for every $j$. Alternatively, the spelling length $h_{1}(W)$ is an integer such that the precise word problem holds for the pair $\left(W, h_{1}(W)\right)$ for the presentation (1.2) in which $E_{i}=\{1\}$ for every $i$.

As a corollary of Theorem 1.2, we obtain a positive solution to the problem of Grigorchuk and Kurchanov and also compute both the width and the spelling length of $W$ in cubic logarithmic space. We remark that Riley [36] gave an independent solution to the Grigorchuk-Kurchanov problem.

Corollary 1.3. Let $W$ be a word over $\mathcal{A}^{ \pm 1}$ and $n \geq 0$ be an integer. Then the decision problems that inquire whether the width $h(W)$ or the spelling length $h_{1}(W)$ of $W$ is equal to $n$ belong to $L^{3}$ and $P$. Specifically, the problems can be solved in deterministic space $O\left((\log |W|)^{3}\right)$ or in deterministic time $O\left(|W|^{4} \log |W|\right)$.

Making many technical modifications but keeping the general strategy of arguments unchanged, we will obtain similar to Theorem 1.2 results for BaumslagSolitar one-relator groups. 
Theorem 1.4. Let the group $\mathcal{G}_{3}$ be defined by a presentation of the form

$$
\mathcal{G}_{3}:=\left\langle a_{1}, \ldots, a_{m} \| a_{2} a_{1}^{n_{1}} a_{2}^{-1}=a_{1}^{n_{2}}\right\rangle
$$

where $n_{1}, n_{2}$ are some nonzero integers. Then both the bounded and precise word problems for (1.4) are in $L^{3}$ and in P. Specifically, the problems can be solved in deterministic space $O\left(\left(\max (\log |W|, \log n)(\log |W|)^{2}\right)\right.$ or in deterministic time $O\left(|W|^{4}\right)$.

As another application of our techniques, we will obtain a solution in polylogarithmic space for the (minimal) diagram problem for presentation (1.2) which includes the case of the free group $\mathcal{F}(\mathcal{A})=\langle\mathcal{A} \| \varnothing\rangle$ over $\mathcal{A}$ without relations. Recall that the diagram problem for a decidable presentation (1.1) is a search problem that, given a word $W$ over $\mathcal{A}^{ \pm 1}$ with $W \stackrel{\underline{\underline{G}}}{1}$, asks to algorithmically construct a disk diagram $\Delta$ over (1.1) whose boundary $\partial \Delta$ is labeled by $W$, denoted $\varphi(\partial \Delta) \equiv W$, for the definitions see Sect. 2. Analogously, the minimal diagram problem for a decidable presentation (1.1) is a search problem that, given a word $W$ over $\mathcal{A}^{ \pm 1}$ with $W \stackrel{\underline{G}}{=} 1$, asks to algorithmically construct a disk diagram $\Delta$ over (1.1) such that $\varphi(\partial \Delta) \equiv W$ and $\Delta$ contains a minimal number of faces.

Recall that, according to Lipton and Zalcstein [16, the word problem for the free group $\mathcal{F}(\mathcal{A})$, given by presentation (1.1) with $\mathcal{R}=\varnothing$, is in L. However, construction of an actual diagram $\Delta$ over $\mathcal{F}(\mathcal{A})$ for a word $W$ over $\mathcal{A}^{ \pm 1}$ such that $\varphi(\partial \Delta) \equiv W$, is a different matter and it is not known whether this construction could be done in polylogarithmic space (note that it is easy to construct such a diagram, which is a tree, in polynomial time). In fact, many results of this article grew out of the attempts to solve the diagram problem for free groups with no relations in subpolynomial space.

Theorem 1.5. Both the diagram problem and the minimal diagram problem for group presentation (1.2) can be solved in deterministic space $O\left((\log |W|)^{3}\right)$ or in deterministic time $O\left(|W|^{4} \log |W|\right)$.

Furthermore, let $W$ be a word such that $W \stackrel{\underline{G}_{2}}{=} 1$ and let

$$
\tau(\Delta)=\left(\tau_{1}(\Delta), \ldots, \tau_{s_{\tau}}(\Delta)\right)
$$

be a tuple of integers, where the absolute value $\left|\tau_{i}(\Delta)\right|$ of each $\tau_{i}(\Delta)$ represents the number of certain vertices or faces in a disk diagram $\Delta$ over (1.2) such that $\varphi(\partial \Delta) \equiv W$. Then, in deterministic space $O\left((\log |W|)^{3}\right)$, one can algorithmically construct such a minimal diagram $\Delta$ which is also smallest relative to the tuple $\tau(\Delta)$ (the tuples are ordered lexicographically).

We point out that the case of the free group $\mathcal{F}(\mathcal{A})=\langle\mathcal{A} \| \varnothing\rangle$ with no relations is covered by Theorem 1.5 and, since there are no relations, every diagram $\Delta$ over $\mathcal{F}(\mathcal{A})=\langle\mathcal{A} \| \varnothing\rangle$ is minimal. Hence, for the free group $\mathcal{F}(\mathcal{A})$, Theorem 1.5 implies the following.

Corollary 1.6. There is a deterministic algorithm that, for given word $W$ over the alphabet $\mathcal{A}^{ \pm 1}$ such that $W^{\mathcal{F}(\mathcal{A})}=1$, where $\mathcal{F}(\mathcal{A})=\langle\mathcal{A} \| \varnothing\rangle$ is the free group over $\mathcal{A}$, constructs a pattern of cancellations of letters in $W$ that result in the empty word and the algorithm operates in space $O\left((\log |W|)^{3}\right)$. 
Furthermore, let $\Delta$ be a disk diagram over $\mathcal{F}(\mathcal{A})$ that corresponds to a pattern of cancellations of letters in $W$, i.e., $\varphi(\partial \Delta) \equiv W$, and let

$$
\tau(\Delta)=\left(\tau_{1}(\Delta), \ldots, \tau_{s_{\tau}}(\Delta)\right)
$$

be a tuple of integers, where the absolute value $\left|\tau_{i}(\Delta)\right|$ of each $\tau_{i}(\Delta)$ represents the number of vertices in $\Delta$ of certain degree. Then, also in deterministic space $O\left((\log |W|)^{3}\right)$, one can algorithmically construct such a diagram $\Delta$ which is smallest relative to the tuple $\tau(\Delta)$.

Here is the analogue of Theorem 1.5) for presentations (1.4) of one-relator BaumslagSolitar groups.

Theorem 1.7. Suppose that $W$ is a word over the alphabet $\mathcal{A}^{ \pm 1}$ such that the bounded word problem for presentation (1.4) holds for the pair $(W, n)$. Then a minimal diagram $\Delta$ over (1.4) such that $\varphi(\partial \Delta) \equiv W$ can be algorithmically constructed in deterministic space $O\left(\max (\log |W|, \log n)(\log |W|)^{2}\right)$ or in deterministic time $O\left(|W|^{4}\right)$.

In addition, if $\left|n_{1}\right|=\left|n_{2}\right|$ in (1.4), then the minimal diagram problem for presentation (1.4) can be solved in deterministic space $O\left((\log |W|)^{3}\right)$ or in deterministic time $O\left(|W|^{3} \log |W|\right)$.

As further applications of the techniques of the proof of Theorems 1.4, 1.7, we obtain computational results on discrete homotopy of polygonal closed curves in the plane.

Let $\mathcal{T}$ denote a tessellation of the plane $\mathbb{R}^{2}$ into unit squares whose vertices are points with integer coordinates. Let $c$ be a finite closed path in $\mathcal{T}$ so that edges of $c$ are edges of $\mathcal{T}$. Consider the following two types of elementary operations over $c$. If $e$ is an oriented edge of $c, e^{-1}$ is the edge with an opposite to $e$ orientation, and $e e^{-1}$ is a subpath of $c$ so that $c=c_{1} e e^{-1} c_{2}$, where $c_{1}, c_{2}$ are subpaths of $c$, then the operation $c \rightarrow c_{1} c_{2}$ over $c$ is called an elementary homotopy of type 1. Suppose that $c=c_{1} u c_{2}$, where $c_{1}, u, c_{2}$ are subpaths of $c$, and a boundary path $\partial s$ of a unit square $s$ of $\mathcal{T}$ is $\partial s=u v$, where $u, v$ are subpaths of $\partial s$ and either of $u, v$ could be of zero length, i.e., either of $u, v$ could be a single vertex of $\partial s$. Then the operation $c \rightarrow c_{1} v^{-1} c_{2}$ over $c$ is called an elementary homotopy of type 2 .

Theorem 1.8. Let $c$ be a finite closed path in a tessellation $\mathcal{T}$ of the plane $\mathbb{R}^{2}$ into unit squares so that edges of $c$ are edges of $\mathcal{T}$. Then a minimal number $m_{2}(c)$ such that there is a finite sequence of elementary homotopies of type 1-2, which turns $c$ into a single point and which contains $m_{2}(c)$ elementary homotopies of type 2, can be computed in deterministic space $O\left((\log |c|)^{3}\right)$ or in deterministic time $O\left(|c|^{3} \log |c|\right)$, where $|c|$ is the length of $c$.

Furthermore, such a sequence of elementary homotopies of type 1-2, which turns $c$ into a single point and which contains $m_{2}(c)$ elementary homotopies of type 2, can also be computed in deterministic space $O\left((\log |c|)^{3}\right)$ or in deterministic time $O\left(|c|^{3} \log |c|\right)$.

We remark that this number $m_{2}(c)$ defined in Theorem 1.8 can be regarded as the area "bounded" by a closed path $c$ in $\mathcal{T}$. Clearly, if $c$ is simple, i.e., $c$ has no self-intersections, then $m_{2}(c)$ is the area of the compact region bounded by $c$. If $c$ is simple then the area bounded by $c$ can be computed in logarithmic space $O(\log |c|)$ as follows from the "shoelace" formula for the area of a simple polygon. 
More generally, assume that $c$ is a continuous closed curve in $\mathbb{R}^{2}$, i.e., $c$ is the image of a continuous map $\mathbb{S}^{1} \rightarrow \mathbb{R}^{2}$, where $\mathbb{S}^{1}$ is a circle. Consider a homotopy $H: \mathbb{S}^{1} \times[0,1] \rightarrow \mathbb{R}^{2}$ that turns the curve $c=H\left(\mathbb{S}^{1} \times\{0\}\right)$ into a point $H\left(\mathbb{S}^{1} \times\{1\}\right)$ so that for every $t, 0 \leq t<1, H\left(\mathbb{S}^{1} \times\{t\}\right)$ is a closed curve. Let $A(H)$ denote the area swept by the curves $H\left(\mathbb{S}^{1} \times\{t\}\right), 0 \leq t<1$, and let $A(c)$ denote the infimum $\inf _{H} A(H)$ over all such homotopies $H$. As above, we remark that this number $A(c)$ can be regarded as the area defined (or "bounded") by $c$. Note that this number $A(c)$ is different from the signed area of $c$ defined by applying the "shoelace" formula to singular polygons.

Other applications that we discuss here involve polygonal (or piecewise linear) closed curves in the plane and computation and approximation of the area defined by these curves in polylogarithmic space or in polynomial time.

We say that $c$ is a polygonal closed curve in the plane $\mathbb{R}^{2}$ with given tessellation $\mathcal{T}$ into unit squares if $c$ consists of finitely many line segments $c_{1}, \ldots, c_{k}, k>0$, whose endpoints are vertices of $\mathcal{T}, c=c_{1} \ldots c_{k}$, and $c$ is closed. If $c_{i} \subset \mathcal{T}$ then the $\mathcal{T}$-length $\left|c_{i}\right|_{\mathcal{T}}$ of $c_{i}$ is the number of edges of $\mathcal{T}$ in $c_{i}$. If $c_{i} \not \subset \mathcal{T}$ then the $\mathcal{T}$-length $\left|c_{i}\right| \mathcal{T}$ of $c_{i}$ is the number of connected components in $c_{i} \backslash \mathcal{T}$. We assume that $\left|c_{i}\right|_{\mathcal{T}}>0$ for every $i$ and set $|c|_{\mathcal{T}}:=\sum_{i=1}^{k}\left|c_{i}\right|_{\mathcal{T}}$.

Theorem 1.9. Suppose that $n \geq 1$ is a fixed integer and $c$ is a polygonal closed curve in the plane $\mathbb{R}^{2}$ with given tessellation $\mathcal{T}$ into unit squares. Then, in deterministic space $O\left(\left(\log |c|_{\mathcal{T}}\right)^{3}\right)$ or in deterministic time $O\left(|c|_{\mathcal{T}}^{n+3} \log |c|_{\mathcal{T}}\right)$, one can compute a rational number $r_{n}$ such that $\left|A(c)-r_{n}\right|<\frac{1}{|c|_{\mathcal{T}}^{n}}$.

In particular, if the area $A(c)$ defined by $c$ is known to be an integer multiple of $\frac{1}{L}$, where $L>0$ is a given integer and $L<|c|_{\mathcal{T}}^{n} / 2$, then $A(c)$ can be computed in deterministic space $O\left(\left(\log |c|_{\mathcal{T}}\right)^{3}\right)$ or in deterministic time $O\left(|c|_{\mathcal{T}}^{n+3} \log |c|_{\mathcal{T}}\right)$.

Corollary 1.10. Let $K \geq 1$ be a fixed integer and let $c$ be a polygonal closed curve in the plane $\mathbb{R}^{2}$ with given tessellation $\mathcal{T}$ into unit squares such that $c$ has one of the following two properties (a)-(b).

(a) If $c_{i}, c_{j}$ are two nonparallel line segments of $c$ then their intersection point, if it exists, has coordinates that are integer multiples of $\frac{1}{K}$.

(b) If $c_{i}$ is a line segment of $c$ and $a_{i, x}, a_{i, y}$ are coprime integers such that the line given by an equation $a_{i, x} x+a_{i, y} y=b_{i}$, where $b_{i}$ is an integer, contains $c_{i}$, then $\max \left(\left|a_{i, x}\right|,\left|a_{i, y}\right|\right) \leq K$.

Then the area $A(c)$ defined by c can be computed in deterministic space $O\left(\left(\log |c|_{\mathcal{T}}\right)^{3}\right)$ or in deterministic time $O\left(|c|_{\mathcal{T}}^{n+3} \log |c|_{\mathcal{T}}\right)$, where $n$ depends on $K$.

In particular, if $\mathcal{T}_{*}$ is a tessellation of the plane $\mathbb{R}^{2}$ into equilateral triangles of unit area, or into regular hexagons of unit area, and $q$ is a finite closed path in $\mathcal{T}_{*}$ whose edges are edges of $\mathcal{T}_{*}$, then the area $A(q)$ defined by $q$ can be computed in deterministic space $O\left((\log |q|)^{3}\right)$ or in deterministic time $O\left(|q|^{5} \log |q|\right)$.

It is tempting to try to lift the restrictions of Corollary 1.10 to be able to compute, in polylogarithmic space, the area $A(c)$ defined by an arbitrary polygonal closed curve $c$ in the plane equipped with a tessellation $\mathcal{T}$ into unit squares. However, in the general situation, this idea would not work because the rational number $A(c)$ might have an exponentially large denominator, hence, $A(c)$ could take polynomial space just to store (let alone the computations), see an example in the end of Sect. 10 . 
We remark in passing that there are decision problems in NP that are not known to be NP-complete or in P, called NP-intermediate problems, that are solvable in polylogarithmic space. For example, a restricted version of the NP-complete clique problem asks whether a graph on $n$ vertices contains a clique with at most $[\log n]$ vertices, where $[k]$ is the integer part of $k$, and this restriction is obviously a problem solvable in nondeterministic space $O\left([\log n]^{2}\right)$. More natural examples of such NP-intermediate problems would be a decision version of the problem on finding a minimum dominating set in a tournament, [24, [33, and the problem on isomorphism of two finite groups given by their multiplication tables, 4], 17.

\section{Preliminaries}

If $U, V$ are words over an alphabet $\mathcal{A}^{ \pm 1}:=\mathcal{A} \cup \mathcal{A}^{-1}$, then $U \stackrel{0}{=} V$ denotes the equality of $U, V$ as elements of the free group $\mathcal{F}(\mathcal{A})$ whose set of free generators is $\mathcal{A}$. The equality of natural images of words $U, V$ in the group $\mathcal{G}$, given by presentation (1.1), is denoted $U \stackrel{\underline{G}}{=} V$.

The letter-by-letter equality of words $U, V$ is denoted $U \equiv V$. If $U \equiv a_{i_{1}}^{\varepsilon_{1}} \ldots a_{i_{\ell}}^{\varepsilon_{\ell}}$, where $a_{i_{1}}, \ldots, a_{i_{\ell}} \in \mathcal{A}$ and $\varepsilon_{1}, \ldots, \varepsilon_{\ell} \in\{ \pm 1\}$, then the length of $U$ is $|U|=\ell$. A nonempty word $U$ over $\mathcal{A}^{ \pm 1}$ is called reduced if $U$ contains no subwords of the form $a a^{-1}, a^{-1} a$, where $a \in \mathcal{A}$.

Let $\Delta$ be a 2-complex and let $\Delta(i)$ denote the set of nonoriented $i$-cells of $\Delta$, $i=0,1,2$. We also consider the set $\vec{\Delta}(1)$ of oriented 1 -cells of $\Delta$. If $e \in \vec{\Delta}(1)$ then $e^{-1}$ denotes $e$ with the opposite orientation, note that $e \neq e^{-1}$. For every $e \in \vec{\Delta}(1)$, let $e_{-}, e_{+}$denote the initial, terminal, resp., vertices of $e$. In particular, $\left(e^{-1}\right)_{-}=e_{+}$and $\left(e^{-1}\right)_{+}=e_{-}$. The closures of $i$-cells of $\Delta$ are called vertices, edges, faces when $i=0,1,2$, resp.

A path $p=e_{1} \ldots e_{\ell}$ in $\Delta$ is a sequence of oriented edges $e_{1}, \ldots, e_{\ell}$ of $\Delta$ such that $\left(e_{i}\right)_{+}=\left(e_{i+1}\right)_{-}, i=1, \ldots, \ell-1$. The length of a path $p=e_{1} \ldots e_{\ell}$ is $|p|=\ell$. The initial vertex of $p$ is $p_{-}:=\left(e_{1}\right)_{-}$and the terminal vertex of $p$ is $p_{+}:=\left(e_{\ell}\right)_{+}$. We allow the possibility that $|p|=0$ and $p=p_{-}$.

A path $p$ is called reduced if $|p|>0$ and $p$ contains no subpath of the form $e e^{-1}$, where $e$ is an edge. A path $p$ is called closed if $p_{-}=p_{+}$.

A cyclic path is a closed path with no distinguished initial vertex. A path $p=e_{1} \ldots e_{\ell}$ is called simple if the vertices $\left(e_{1}\right)_{-}, \ldots,\left(e_{\ell}\right)_{-},\left(e_{\ell}\right)_{+}$are all distinct. A closed path $p=e_{1} \ldots e_{\ell}$ is simple if the vertices $\left(e_{1}\right)_{-}, \ldots,\left(e_{\ell}\right)_{-}$are all distinct.

A disk diagram, also called a van Kampen diagram, $\Delta$ over a presentation (1.1) is a planar connected and simply connected finite 2-complex which is equipped with a labeling function

$$
\varphi: \vec{\Delta}(1) \rightarrow \mathcal{A} \cup \mathcal{A}^{-1}=\mathcal{A}^{ \pm 1}
$$

such that, for every $e \in \vec{\Delta}(1), \varphi\left(e^{-1}\right)=\varphi(e)^{-1}$ and, for every face $\Pi$ of $\Delta$, if $\partial \Pi=e_{1} \ldots e_{\ell}$ is a boundary path of $\Pi$, where $e_{1}, \ldots, e_{\ell} \in \vec{\Delta}(1)$, then

$$
\varphi(\partial \Pi):=\varphi\left(e_{1}\right) \ldots \varphi\left(e_{\ell}\right)
$$

is a cyclic permutation of one of the words in $\mathcal{R}^{ \pm 1}=\mathcal{R} \cup \mathcal{R}^{-1}$.

A disk diagram $\Delta$ over presentation (1.1) is always considered with an embedding $\Delta \rightarrow \mathbb{R}^{2}$ into the plane $\mathbb{R}^{2}$. This embedding makes it possible to define positive (=counterclockwise) and negative (=clockwise) orientations for boundaries of faces of $\Delta$, for the boundary path $\partial \Delta$ of $\Delta$, and, more generally, for boundaries of disk subdiagrams of $\Delta$. It is convenient to assume, as in [15], [30, that the boundary 
path $\partial \Pi$ of every face $\Pi$ of a disk diagram $\Delta$ has the positive orientation while the boundary path $\partial \Delta$ of $\Delta$ has the negative orientation.

If $o \in \partial \Delta$ is a vertex, let $\left.\partial\right|_{o} \Delta$ denote a boundary path of $\Delta$ (negatively oriented) starting (and ending) at $o$. Using this notation, we now state van Kampen lemma on geometric interpretation of consequences of defining relations.

Lemma 2.1. Let $W$ be a nonempty word over $\mathcal{A}^{ \pm 1}$ and let a group $\mathcal{G}$ be defined by presentation (1.1). Then $W \stackrel{\underline{\underline{\mathcal{G}}}}{1}$ if and only if there is a disk diagram over presentation (1.1) such that $\varphi\left(\left.\partial\right|_{o} \Delta\right) \equiv W$ for some vertex $o \in \partial \Delta$.

Proof. The proof is straightforward, for details the reader is referred to [15], [30], see also [18.

A disk diagram $\Delta$ over presentation (1.1) with $\varphi(\partial \Delta) \equiv W$ is called minimal if $\Delta$ contains a minimal number of faces among all disk diagrams $\Delta^{\prime}$ such that $\varphi\left(\partial \Delta^{\prime}\right) \equiv W$.

A disk diagram $\Delta$ over (1.1) is called reduced if $\Delta$ contains no two faces $\Pi_{1}, \Pi_{2}$ such that there is a vertex $v \in \partial \Pi_{1}, v \in \partial \Pi_{2}$ and the boundary paths $\left.\partial\right|_{v} \Pi_{1},\left.\partial\right|_{v} \Pi_{2}$ of the faces, starting at $v$, satisfy $\varphi\left(\left.\partial\right|_{v} \Pi_{1}\right) \equiv \varphi\left(\left.\partial\right|_{v} \Pi_{2}\right)^{-1}$. If $\Delta$ is not reduced and $\Pi_{1}, \Pi_{2}$ is a pair of faces that violates the definition of being reduced for $\Delta$, then these faces $\Pi_{1}, \Pi_{2}$ are called a reducible pair (cf. similar definitions in [15], 18, 30]). A reducible pair of faces $\Pi_{1}, \Pi_{2}$ can be removed from $\Delta$ by a surgery that cuts through the vertex $v$ and identifies the boundary paths $\left.\partial\right|_{v} \Pi_{1}$ and $\left(\left.\partial\right|_{v} \Pi_{2}\right)^{-1}$, see Fig. 2.1, more details can be found in [18, 30]. As a result, one obtains a disk diagram $\Delta^{\prime}$ such that $\varphi\left(\partial \Delta^{\prime}\right) \equiv \varphi(\partial \Delta)$ and $\left|\Delta^{\prime}(2)\right|=|\Delta(2)|-2$, where $|\Delta(2)|$ is the number of faces in $\Delta$. In particular, a minimal disk diagram is always reduced.

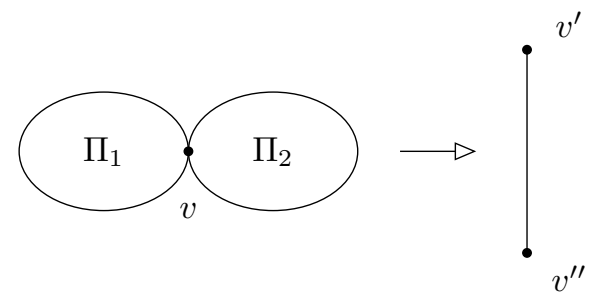

Fig. 2.1

Note that if $o \in \partial \Delta$ is a vertex and $\left.\partial\right|_{o} \Delta=q_{1} q_{2}$ is a factorization of the boundary path $\left.\partial\right|_{o} \Delta$ of $\Delta, q_{1}$ is closed, $0<\left|q_{1}\right|,\left|q_{2}\right|<|\partial \Delta|$, then the notation $\left.\partial\right|_{o} \Delta$ is in fact ambiguous, because the path $q_{2} q_{1}$ also has the form $\left.\partial\right|_{o} \Delta$. To avoid this (and other) type of ambiguity, for a given pair $(W, \Delta)$, where $W \stackrel{\underline{g}}{=} 1$ and $\Delta$ is a disk diagram for $W$ as in Lemma 2.1 we consider a "model" simple path $P_{W}$ such that $\left|P_{W}\right|=|W|$, $P_{W}$ is equipped with a labeling function $\varphi: \vec{P}_{W}(1) \rightarrow \mathcal{A}^{ \pm 1}$ on the set $\vec{P}_{W}(1)$ of its oriented edges so that $\varphi\left(e^{-1}\right)=\varphi(e)^{-1}$ and $\varphi\left(P_{W}\right) \equiv W$.

It will be convenient to identify vertices of $P_{W}$ with integers $0,1, \ldots,|W|$ so that $\left(P_{W}\right)_{-}=0$ and the numbers corresponding to vertices increase by one as one goes along $P_{W}$ from $\left(P_{W}\right)_{-}=0$ to $\left(P_{W}\right)_{+}=|W|$. This makes it possible to compare vertices $v_{1}, v_{2} \in P_{W}(0)$ by the standard order $\leq$ defined on the integers, consider vertices $v_{1} \pm 1$ etc.

For a given pair $(W, \Delta)$, where $W \stackrel{\underline{\underline{G}}}{=}$, let

$$
\alpha: P_{W} \rightarrow \partial \Delta
$$


be a continuous cellular map that preserves dimension of cells, $\varphi$-labels of edges, and has the property that $\alpha\left(\left(P_{W}\right)_{-}\right)=\alpha(0)=o$, where $o$ is a fixed vertex of $\partial \Delta$ with $\varphi\left(\left.\partial\right|_{o} \Delta\right) \equiv W$.

If $v$ is a vertex of $P_{W}$, let

$$
P_{W}(\text { fact }, v)=p_{1} p_{2}
$$

denote the factorization of $P_{W}$ defined by $v$ so that $\left(p_{1}\right)_{+}=v$. Analogously, if $v_{1}, v_{2}$ are vertices of $P_{W}$ with $v_{1} \leq v_{2}$, we let

$$
P_{W}\left(\text { fact }, v_{1}, v_{2}\right)=p_{1} p_{2} p_{3}
$$

denote the factorization of $P_{W}$ defined by $v_{1}, v_{2}$ so that $\left(p_{2}\right)_{-}=v_{1}$ and $\left(p_{2}\right)_{+}=v_{2}$. Note that if $v_{1}=v_{2}$, then $p_{2}=\left\{v_{1}\right\}$ and $\left|p_{2}\right|=0$. Clearly, $\left|p_{2}\right|=v_{2}-v_{1}$.

Making use of the introduced notation, consider a vertex $v$ of $P_{W}$ and let $P_{W}($ fact, $v)=p_{1} p_{2}$. Define $\left.\partial\right|_{v} \Delta:=\alpha\left(p_{2}\right) \alpha\left(p_{1}\right)$. This notation $\left.\partial\right|_{v} \Delta$, in place of $\left.\partial\right|_{\alpha(v)} \Delta$, will help us to avoid the potential ambiguity when writing $\left.\partial\right|_{\alpha(v)} \Delta$. In particular, if $\bar{W}$ is a cyclic permutation of $W$ so that the first $k$, where $0 \leq k \leq|W|-1$, letters of $W$ are put at the end of $W$, then $\varphi\left(\left.\partial\right|_{k} \Delta\right) \equiv \bar{W}$. It is clear that $\varphi\left(\left.\partial\right|_{0} \Delta\right) \equiv W$.

Consider the following property.

(A) Suppose that $\Delta$ is a disk diagram over (1.2). If $\Pi$ is a face of $\Delta$ and $e \in \partial \Pi$ is an edge then $e^{-1} \in \partial \Delta$.

We now state a lemma in which we record some simple properties of disk diagrams over (1.2) related to property (A).

Lemma 2.2. Let $\Delta$ be a disk diagram over presentation (1.2). Then the following hold true.

(a) If $\Delta$ has property (A), then the degree of every vertex of $\Delta$ is at most $2|\partial \Delta|$, the boundary path $\partial \Pi$ of every face $\Pi$ of $\Delta$ is simple, and

$$
|\Delta(2)| \leq|\partial \Delta|, \quad \sum_{\Pi \in \Delta(2)}|\partial \Pi| \leq|\partial \Delta| .
$$

(b) There exists a disk diagram $\Delta^{\prime}$ over (1.2) such that $\varphi\left(\partial \Delta^{\prime}\right) \equiv \varphi(\partial \Delta)$, $\left|\Delta^{\prime}(2)\right| \leq|\Delta(2)|$ and $\Delta^{\prime}$ has property (A).

Proof. (a) Let $v$ be a vertex of $\Delta$. By property (A), $v \in \partial \Delta$ and if $e$ is an edge such that $e_{-}=v$, then either $e \in \partial \Delta$ or $e \in \partial \Pi$, where $\Pi$ is a face of $\Delta$, and $e^{-1} \in \partial \Delta$. This implies that $\operatorname{deg} v \leq 2|\partial \Delta|$.

If the boundary path $\partial \Pi$ of a face $\Pi$ of $\Delta$ is not simple, then there is a factorization $\partial \Pi=u_{1} u_{2}$, where $u_{1}, u_{2}$ are closed subpaths of $\partial \Pi$ and $0<\left|u_{1}\right|,\left|u_{2}\right|<|\partial \Pi|$, see Fig. 2.2. Clearly, the edges of one of the paths $u_{1}, u_{2}$ do not belong to the boundary $\partial \Delta$ of $\Delta$, contrary to property (A) of $\Delta$.

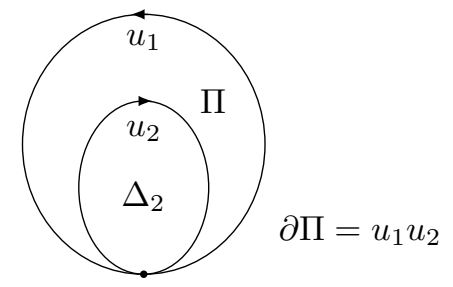

Fig. 2.2 
The inequality $|\Delta(2)| \leq|\partial \Delta|$ and its stronger version $\sum_{\Pi \in \Delta(2)}|\partial \Pi| \leq|\partial \Delta|$ are immediate from property (A).

(b) Suppose that a disk diagram $\Delta$ over (1.2) does not have property (A).

First assume that $\Delta$ contains a face $\Pi$ whose boundary path $\partial \Pi$ is not simple. Then, as in the proof of part (a), there is a factorization $\partial \Pi=u_{1} u_{2}$, where $u_{1}, u_{2}$ are closed subpaths of $\partial \Pi$ and $0<\left|u_{1}\right|,\left|u_{2}\right|<|\partial \Pi|$. Renaming $u_{1}$ and $u_{2}$ if necessary, we may assume that $u_{2}$ bounds a disk subdiagram $\Delta_{2}$ of $\Delta$ such that $\Delta_{2}$ does not contain $\Pi$, see Fig. 2.2. If $u_{2}$ is not simple, then we can replace $u_{2}$ by its closed subpath $u_{2}^{\prime}$ such that $0<\left|u_{2}^{\prime}\right|<\left|u_{2}\right|$ and $u_{2}^{\prime}$ bounds a disk subdiagram $\Delta_{2}^{\prime}$ that contains no $\Pi$. Hence, choosing a shortest path $u_{2}$ as above, we may assume that $u_{2}$ is simple. By Lemma 2.1 applied to $\Delta_{2}$ with $\partial \Delta_{2}=u_{2}$, we have

$$
\varphi\left(u_{2}\right) \equiv \varphi\left(\partial \Delta_{2}\right) \stackrel{\mathcal{G}_{2}}{=} 1
$$

Denote $\varphi(\partial \Pi) \equiv a_{i}^{\varepsilon k}$, where $\varepsilon= \pm 1, k \in E_{i}$.

Note that the group $\mathcal{G}_{2}$ is the free product of cyclic groups generated by the images of generators $a_{1}, \ldots, a_{m}$ and the image of $a_{j}$ has order $n_{j}>0$ if $E_{j} \neq\{0\}$ or the image of $a_{j}$ has infinite order if $E_{j}=\{0\}$. Hence, an equality $a_{j}^{\ell} \stackrel{\mathcal{G}_{2}}{=} 1$, where $\ell \neq 0$, implies that $E_{j} \neq\{0\}$ and $n_{j}$ divides $\ell$.

It follows from (2.1) and $0<\left|u_{2}\right|<|\partial \Pi|$ that $\varphi\left(u_{2}\right) \equiv a_{i}^{\varepsilon k_{2}}$, where $k_{2} \in E_{i}$, $k_{2}<k$. Therefore, $E_{i}=\left\{n_{i}, 2 n_{i}, \ldots\right\}$ and $\varphi\left(u_{1}\right) \equiv a_{i}^{\varepsilon\left(k-k_{2}\right)}$, where $k-k_{2} \in E_{i}$. Hence, we can consider a face $\Pi^{\prime}$ such that $\varphi\left(\partial \Pi^{\prime}\right) \equiv \varphi\left(u_{1}\right) \equiv a_{i}^{\varepsilon\left(k-k_{2}\right)}$. Now we take the subdiagram $\Delta_{2}$ out of $\Delta$ and replace the face $\Pi$ with $\varphi(\partial \Pi) \equiv a_{i}^{\varepsilon k}$ by the face $\Pi^{\prime}$ with $\varphi\left(\partial \Pi^{\prime}\right) \equiv a_{i}^{\varepsilon\left(k-k_{2}\right)}$. Doing this results in a disk diagram $\Delta^{\prime}$ such that $\varphi\left(\partial \Delta^{\prime}\right) \equiv \varphi\left(\partial \Delta^{\prime}\right)$ and $\left|\Delta^{\prime}(2)\right|<|\Delta(2)|$ as $\left|\Delta_{2}(2)\right|>0$.

Assume that, for every face $\Pi$ in $\Delta$, the boundary path $\partial \Pi$ is simple. Also, assume that the property (A) fails for $\Delta$. Then there are faces $\Pi_{1}, \Pi_{2}, \Pi_{1} \neq \Pi_{2}$, and an edge $e$ such that $e \in \partial \Pi_{1}$ and $e^{-1} \in \partial \Pi_{2}$. Consider a disk subdiagram $\Gamma$ of $\Delta$ that contains $\Pi_{1}, \Pi_{2}$ and $\Gamma$ is minimal with this property relative to $|\Gamma(2)|+|\Gamma(1)|$. Since $\partial \Pi_{1}, \partial \Pi_{2}$ are simple paths, it follows that $\partial \Gamma=r_{1} r_{2}$, where $r_{1}^{-1}$ is a subpath of $\partial \Pi_{1}$ and $r_{2}^{-1}$ is a subpath of $\partial \Pi_{2}$. Denote $\varphi\left(\partial \Pi_{1}\right) \equiv a_{i}^{\varepsilon_{1} k_{1}}$, where $\varepsilon_{1}= \pm 1$ and $k_{1} \in E_{i}$. Clearly, $\varphi\left(\partial \Pi_{2}\right) \equiv a_{i}^{-\varepsilon_{1} k_{2}}$, where $k_{2} \in E_{i}$ and

$$
\varphi(\partial \Gamma) \equiv \varphi\left(r_{1}\right) \varphi\left(r_{2}\right) \stackrel{0}{=} a_{i}^{\varepsilon k},
$$

where $\varepsilon= \pm 1$ and $k \geq 0$. As above, we observe that $k \in E_{i}$ following from $a_{i}^{\varepsilon k} \stackrel{\underline{\mathcal{G}_{2}}}{=} 1$. Hence, we may consider a disk diagram $\Gamma^{\prime}$ such that $\partial \Gamma^{\prime}=r_{1}^{\prime} r_{2}^{\prime}$, where $\varphi\left(r_{1}^{\prime}\right) \equiv$ $\varphi\left(r_{1}\right), \varphi\left(r_{2}^{\prime}\right) \equiv \varphi\left(r_{2}\right)$, and $\Gamma^{\prime}$ contains a single face $\Pi$ such that $\varphi(\partial \Pi) \equiv a_{i}^{-\varepsilon k}$ if $k \neq 0$ or $\Gamma^{\prime}$ contains no faces if $k=0$. We take the subdiagram $\Gamma$ out of $\Delta$ and replace $\Gamma$ with $\Gamma^{\prime}$, producing thereby a disk diagram $\Delta^{\prime}$ such that $\varphi\left(\partial \Delta^{\prime}\right) \equiv \varphi\left(\partial \Delta^{\prime}\right)$ and $\left|\Delta^{\prime}(2)\right|<|\Delta(2)|$.

We now observe that if property (A) fails for $\Delta$ then there is a face $\Pi$ in $\Delta$ such that $\partial \Pi$ is not simple or there are distinct faces $\Pi_{1}, \Pi_{2}$ and an edge $e$ such that $e \in \partial \Pi_{1}$ and $e^{-1} \in \partial \Pi_{2}$. In either case, as was shown above, we can find a disk diagram $\Delta^{\prime}$ such that $\varphi\left(\partial \Delta^{\prime}\right) \equiv \varphi\left(\partial \Delta^{\prime}\right)$ and $\left|\Delta^{\prime}(2)\right|<|\Delta(2)|$. Now obvious induction on $|\Delta(2)|$ completes the proof of part (b).

In view of Lemma 2.2(b), we will be assuming in Sects. $4-5,8$ that if $\Delta$ is a disk diagram over presentation (1.2), then $\Delta$ has property (A). 


\section{Proof of Proposition 1.1}

Proposition 1.1. (a) There exists a decidable group presentation (1.1) for which the word problem is solvable while the bounded and precise word problems are not solvable.

(b) If the bounded word problem is solvable for (1.1), then the precise word problem is also solvable.

(c) For every finite group presentation (1.1), the bounded word problem is in $N P$, i.e., it can be solved in nondeterministic polynomial time, and the precise word problem is in PSPACE, i.e., it can be solved in polynomial space.

(d) There exists a finite group presentation (1.1) for which the bounded and precise word problems are solvable while the word problem is not solvable.

(e) There exists a finitely presented group (1.1) for which the bounded word problem is NP-complete and the precise word problem is NP-hard.

Proof. (a) We will use the construction of [12, Example 3] suggested by C. Jockush and I. Kapovich. Consider the group presentation

$$
\left\langle a, b \| a^{i}=1, a^{i} b^{k_{i}}=1, \quad i \in \mathbb{N}\right\rangle,
$$

where $\mathbb{K}=\left\{k_{1}, k_{2}, \ldots\right\}$ is a recursively enumerable but not recursive subset of the set of natural numbers $\mathbb{N}$ with the indicated enumeration and $k_{1}=1$. It is clear that the set of relations is decidable and this presentation defines the trivial group, hence the word problem is solvable for (3.1). On the other hand, it is easy to see that the bounded word problem for a pair $\left(b^{k}, 2\right)$, where $k \in \mathbb{N}$, holds true if and only if $k \in \mathbb{K}$. Analogously, the precise word problem for a pair $\left(b^{k}, 2\right)$ holds true if and only if $k \in \mathbb{K}$. Since the set $\mathbb{K}$ is not recursive, it follows that both the bounded word problem and the precise word problem for presentation (3.1) are unsolvable.

(b) Note that the precise word problem holds true for a pair $(W, n)$ if and only if the bounded word problem is true for $(W, n)$ and the bounded word problem is false for $(W, n-1)$. This remark means that the solvability of the bounded word problem for (1.1) implies the solvability of the precise word problem for (1.1). On the other hand, the bounded word problem holds for a pair $(W, n)$ if and only if the precise word problem holds for $(W, k)$ with some $k \leq n$. This remark means that the solvability of the precise word problem for (1.1) implies the solvability of the bounded word problem for (1.1), as required.

(c) Suppose that presentation (1.1) is finite, i.e., both $\mathcal{A}$ and $\mathcal{R}$ are finite, and we are given a pair $\left(W, 1^{n}\right)$. It follows from definitions and Lemma2.1 that the bounded word problem holds for the pair $\left(W, 1^{n}\right)$ if and only if there is a disk diagram $\Delta$ such that $\varphi(\partial \Delta) \equiv W$ and $|\Delta(2)| \leq n$. Observe that $|\vec{\Delta}(1)| \leq M n+|W|$, where $M=\max \{|R|: R \in \mathcal{R}\}$ is a constant. Therefore, the size of a disk diagram $\Delta$ with $\varphi(\partial \Delta) \equiv W$ is bounded by a linear function in $n+|W|$ and such a diagram $\Delta$ can be used as a certificate to verify in polynomial time that the bounded word problem holds for the pair $\left(W, 1^{n}\right)$. Thus the bounded word problem for finite presentation (1.1) is in NP.

Recall that the precise word problem holds for a pair $\left(W, 1^{n}\right)$ if and only if the bounded word problem is true for $\left(W, 1^{n}\right)$ and the bounded word problem is false for $\left(W, 1^{n-1}\right)$. As we saw above, the bounded word problem for (1.1) is in NP, hence, the complement of the bounded word problem for (1.1) is in coNP. Since 
both coNP and NP are subsets of PSPACE, it follows that the precise word problem for finite presentation (1.1) is in PSPACE.

(d) According to Boone [6, [7] and Novikov [25, 26, see also [18, there exists a finite group presentation (1.1) such that the word problem for this presentation is not solvable. In view of part (c) both the bounded word problem and precise word problem for this presentation are solvable.

(e) According to Birget, Sapir, Ol'shanskii and Rips [5], there exists a finite group presentation (1.1) whose isoperimetric function is bounded by a polynomial $p(x)$ and for which the word problem is NP-complete. It follows from definitions that if $W \stackrel{\underline{G}}{=} 1$ and $\Delta$ is a minimal diagram over presentation (1.1) such that $\varphi(\partial \Delta) \equiv W$ then $|\Delta(2)| \leq p(|W|)$. Therefore, the bounded word problem, whose input is $\left(W, 1^{n}\right)$, where $n \geq p(|W|)$, is equivalent to the word problem, whose input is $W$. Since the latter problem is NP-complete, it follows that the bounded word problem for (1.1) is NP-hard. By part (c), the bounded word problem for (1.1) is in NP, whence the bounded word problem for (1.1) is NP-complete.

Note that the word problem for given word $W$ is equivalent to the disjunction of the claims that the precise word problem holds for the pairs $\left(W, 1^{1}\right),\left(W, 1^{2}\right), \ldots$, $\left(W, 1^{p(|W|)}\right)$. Since $p(x)$ is a polynomial, it follows that the precise word problem for this presentation (1.1) is NP-hard.

\section{Calculus of Brackets for Group Presentation (1.2)}

As in Theorem 1.2 consider a group presentation of the form

$$
\mathcal{G}_{2}=\left\langle a_{1}, \ldots, a_{m} \| a_{i}^{k_{i}}=1, k_{i} \in E_{i}, i=1, \ldots, m\right\rangle,
$$

where, for every $i$, one of the following holds: $E_{i}=\{0\}$, or, for some integer $n_{i}>0$, $E_{i}=\left\{n_{i}\right\}$, or $E_{i}=n_{i} \mathbb{N}=\left\{n_{i}, 2 n_{i}, 3 n_{i}, \ldots\right\}$.

Suppose that $W$ is a nonempty word over $\mathcal{A}^{ \pm 1}, W \stackrel{\mathcal{G}_{2}}{=} 1$ and $\Delta$ is a disk diagram over presentation (1.2) such that $\varphi\left(\left.\partial\right|_{0} \Delta\right) \equiv W$ and $\Delta$ has property (A). Recall that the existence of such a diagram $\Delta$ follows from Lemmas 2.12 .2 (b).

Lemma 4.1. Suppose that $\Delta$ is a disk diagram over presentation (1.2) and $\Delta$ contains no faces, i.e., $\Delta$ is a tree or, equivalently, $\Delta$ is a disk diagram over presentation $F(\mathcal{A})=\left\langle a_{1}, \ldots, a_{m} \| \varnothing\right\rangle$ of the free group $F(\mathcal{A})$ with no relations, and assume that $\varphi\left(\left.\partial\right|_{0} \Delta\right) \equiv W$, where $|W|>2$. Then there are vertices $v_{1}, v_{2} \in P_{W}$ such that $v_{1}<v_{2}, \alpha\left(v_{1}\right)=\alpha\left(v_{2}\right)$ and if $P_{W}\left(\right.$ fact, $\left.v_{1}, v_{2}\right)=p_{1} p_{2} p_{3}$ is the factorization of $P_{W}$ defined by $v_{1}, v_{2}$, then

$$
\min \left(\left|p_{2}\right|,\left|p_{1}\right|+\left|p_{3}\right|\right) \geq \frac{1}{3}|\partial|_{0} \Delta\left|=\frac{1}{3}\right| W \mid .
$$

Proof. It is easy to verify that if $|W| \leq 6$, then Lemma 4.1 is true. Hence, we may assume that $|W|>6$.

For every pair $v_{1}^{\prime}, v_{2}^{\prime}$ of vertices of $P_{W}$ such that $v_{1}^{\prime}<v_{2}^{\prime}$ and $\alpha\left(v_{1}^{\prime}\right)=\alpha\left(v_{2}^{\prime}\right)$, consider the factorization $P_{W}\left(\right.$ fact, $\left.v_{1}^{\prime}, v_{2}^{\prime}\right)=p_{1}^{\prime} p_{2}^{\prime} p_{3}^{\prime}$ and pick such a pair that maximizes $\min \left(\left|p_{2}^{\prime}\right|,\left|p_{1}^{\prime}\right|+\left|p_{3}^{\prime}\right|\right)$. Let $v_{1}, v_{2}$ be such a maximal pair and denote $P_{W}\left(\mathrm{fact}, v_{1}, v_{2}\right)=p_{1} p_{2} p_{3}$. Arguing on the contrary, assume that

$$
\min \left(\left|p_{2}\right|,\left|p_{1}\right|+\left|p_{3}\right|\right)<\frac{1}{3}|W| \text {. }
$$


Denote $q_{i}=\alpha\left(p_{i}\right), i=1,2,3$. Let $e_{1}, \ldots, e_{k}, f_{1}, \ldots, f_{\ell}, k, \ell \geq 1$, be all edges that start at the vertex $\alpha\left(v_{1}\right)=\alpha\left(v_{2}\right)$ so that

$$
q_{2}=e_{1} s_{1} e_{1}^{-1} \ldots e_{k} s_{k} e_{k}^{-1}, \quad q_{3} q_{1}=f_{1} t_{1} f_{1}^{-1} \ldots f_{\ell} t_{\ell} f_{\ell}^{-1},
$$

where $s_{1}, \ldots, s_{k}$ and $t_{1}, \ldots, t_{\ell}$ are subpaths of $q_{2}$ and $q_{3} q_{1}$, resp., see Fig. 4.1.

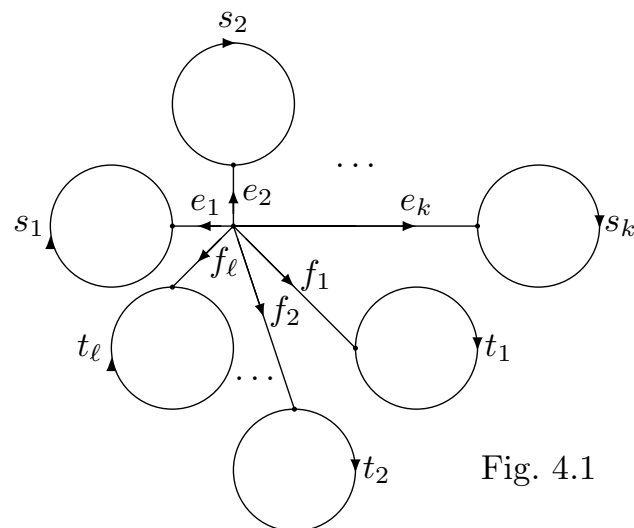

First we assume that $\left|p_{2}\right| \geq\left|p_{1}\right|+\left|p_{3}\right|$. Then, in view of inequality (4.1),

$$
\left|p_{2}\right|>\frac{2}{3}|W|, \quad\left|p_{1}\right|+\left|p_{3}\right|<\frac{1}{3}|W| .
$$

Suppose that for some $i$ we have

$$
\left|e_{i} s_{i} e_{i}^{-1}\right| \geq \frac{1}{2}|W| \text {. }
$$

Pick vertices $v_{1}^{\prime}, v_{2}^{\prime} \in P_{W}$ for which if $P_{W}\left(\right.$ fact, $\left.v_{1}^{\prime}, v_{2}^{\prime}\right)=p_{1}^{\prime} p_{2}^{\prime} p_{3}^{\prime}$ then $\alpha\left(p_{2}^{\prime}\right)=$ $e_{i} s_{i} e_{i}^{-1}$. If $k>1$, then $\left|e_{i} s_{i} e_{i}^{-1}\right|<\left|p_{2}\right|$ and we have a contradiction to the maximality of the pair $v_{1}, v_{2}$ because $\alpha\left(v_{1}^{\prime}\right)=\alpha\left(v_{2}^{\prime}\right)$. Hence, $k=1$ and $i=1$.

Now we pick vertices $v_{1}^{\prime}, v_{2}^{\prime} \in P_{W}$ for which if $P_{W}\left(\right.$ fact, $\left.v_{1}^{\prime}, v_{2}^{\prime}\right)=p_{1}^{\prime} p_{2}^{\prime} p_{3}^{\prime}$ then $\alpha\left(p_{2}^{\prime}\right)=s_{1}$. Note $\left|p_{2}^{\prime}\right|=\left|s_{1}\right|=\left|p_{2}\right|-2>\frac{2}{3}|W|-2 \geq \frac{1}{3}|W|$ for $|W|>6$ and $\left|p_{1}^{\prime}\right|+\left|p_{3}^{\prime}\right|=\left|p_{1}\right|+\left|p_{3}\right|+2<\frac{1}{3}|W|+2 \leq \frac{2}{3}|W|$ for $|W|>6$. Hence, either

$$
\min \left(\left|p_{2}^{\prime}\right|,\left|p_{1}^{\prime}\right|+\left|p_{3}^{\prime}\right|\right) \geq \frac{1}{3}|W|
$$

if $\left|p_{2}^{\prime}\right| \leq\left|p_{1}^{\prime}\right|+\left|p_{3}^{\prime}\right|$ or

$$
\min \left(\left|p_{2}^{\prime}\right|,\left|p_{1}^{\prime}\right|+\left|p_{3}^{\prime}\right|\right)>\min \left(\left|p_{2}\right|,\left|p_{1}\right|+\left|p_{3}\right|\right)
$$

if $\left|p_{2}^{\prime}\right|>\left|p_{1}^{\prime}\right|+\left|p_{3}^{\prime}\right|$. In either case, we obtain a contradiction to the maximality of the pair $v_{1}, v_{2}$ because $\alpha\left(v_{1}^{\prime}\right)=\alpha\left(v_{2}^{\prime}\right)$. Thus it is shown that the inequality (4.3) is false, hence, for every $i=1, \ldots, k$, we have $\left|e_{i} s_{i} e_{i}^{-1}\right|<\frac{1}{2}|W|$.

Assume $\left|e_{i} s_{i} e_{i}^{-1}\right| \geq \frac{1}{3}|W|$ for some $i$. Pick vertices $v_{1}^{\prime}, v_{2}^{\prime} \in P_{W}$ for which if $P_{W}\left(\right.$ fact, $\left.v_{1}^{\prime}, v_{2}^{\prime}\right)=p_{1}^{\prime} p_{2}^{\prime} p_{3}^{\prime}$ then $\alpha\left(p_{2}^{\prime}\right)=e_{i} s_{i} e_{i}^{-1}$. Since $\left|e_{i} s_{i} e_{i}^{-1}\right|<\frac{1}{2}|W|$, it follows that $\min \left(\left|p_{2}^{\prime}\right|,\left|p_{1}^{\prime}\right|+\left|p_{3}^{\prime}\right|\right)=\left|p_{2}^{\prime}\right| \geq \frac{1}{3}|W|$. A contradiction to the maximality of the pair $v_{1}, v_{2}$ proves that $\left|e_{i} s_{i} e_{i}^{-1}\right|<\frac{1}{3}|W|$ for every $i=1, \ldots, k$. According to (4.2), $\left|p_{2}\right|>\frac{2}{3}|W|$, hence, $k \geq 3$ and, for some $i \geq 2$, we obtain

$$
\frac{1}{3}|W| \leq\left|e_{1} s_{1} e_{1}^{-1} \ldots e_{i} s_{i} e_{i}^{-1}\right| \leq \frac{2}{3}|W| .
$$

This means the existence of vertices $v_{1}^{\prime}, v_{2}^{\prime} \in P_{W}$ for which, if

$$
P_{W}\left(\text { fact, } v_{1}^{\prime}, v_{2}^{\prime}\right)=p_{1}^{\prime} p_{2}^{\prime} p_{3}^{\prime},
$$


then the paths $p_{1}^{\prime}, p_{2}^{\prime}, p_{3}^{\prime}$ have the properties that $\alpha\left(p_{2}^{\prime}\right)=e_{1} s_{1} e_{1}^{-1} \ldots e_{i} s_{i} e_{i}^{-1}$ and

$$
\min \left(\left|p_{2}^{\prime}\right|,\left|p_{1}^{\prime}\right|+\left|p_{3}^{\prime}\right|\right) \geq \frac{1}{3}|W| .
$$

This contradiction to the maximality of the pair $v_{1}, v_{2}$ completes the first main case $\left|p_{2}\right| \geq\left|p_{1}\right|+\left|p_{3}\right|$.

Now assume that $\left|p_{2}\right|<\left|p_{1}\right|+\left|p_{3}\right|$. In this case, we repeat the above arguments with necessary changes. By the inequality (4.1), $\left|p_{2}\right|<\frac{1}{3}|W|$ and $\left|p_{1}\right|+\left|p_{3}\right|>\frac{2}{3}|W|$. Suppose that for some $j$ we have

$$
\left|f_{j} t_{j} f_{j}^{-1}\right| \geq \frac{1}{2}|W|
$$

Pick vertices $v_{1}^{\prime}, v_{2}^{\prime} \in P_{W}$ so that $\alpha\left(v_{1}^{\prime}\right)=\alpha\left(v_{2}^{\prime}\right), v_{1}^{\prime}<v_{2}^{\prime}$ and, if

$$
P_{W}\left(\text { fact }, v_{1}^{\prime}, v_{2}^{\prime}\right)=p_{1}^{\prime} p_{2}^{\prime} p_{3}^{\prime},
$$

then either $\alpha\left(p_{2}^{\prime}\right)=f_{j} t_{j} f_{j}^{-1}$ in case when $f_{j} t_{j} f_{j}^{-1}$ is a subpath of one of $q_{1}, q_{3}$, or $\alpha\left(p_{3}^{\prime}\right) \alpha\left(p_{1}^{\prime}\right)=f_{j} t_{j} f_{j}^{-1}$ in case when $f_{j} t_{j} f_{j}^{-1}$ has common edges with both $q_{1}$ and $q_{3}$. In either case,

$$
\min \left(\left|p_{2}^{\prime}\right|,\left|p_{1}^{\prime}\right|+\left|p_{3}^{\prime}\right|\right)>\min \left(\left|p_{2}\right|,\left|p_{1}\right|+\left|p_{3}\right|\right)
$$

whenever $\ell>1$. By the maximality of the pair $v_{1}, v_{2}$, we conclude that $\ell=1$ and $j=1$.

In the case $\ell=j=1$, we consider two subcases: $\min \left(\left|p_{1}\right|,\left|p_{3}\right|\right)>0$ and $\min \left(\left|p_{1}\right|,\left|p_{3}\right|\right)=0$.

Assume that $\min \left(\left|p_{1}\right|,\left|p_{3}\right|\right)>0$. Then we can pick vertices $v_{1}^{\prime}, v_{2}^{\prime} \in P_{W}$ for which, if $P_{W}\left(\right.$ fact, $\left.v_{1}^{\prime}, v_{2}^{\prime}\right)=p_{1}^{\prime} p_{2}^{\prime} p_{3}^{\prime}$, then the subpaths $p_{1}^{\prime}, p_{2}^{\prime}, p_{3}^{\prime}$ of $P_{W}$ have the properties that $\alpha\left(p_{2}^{\prime}\right)=f_{1}^{-1} q_{2} f_{1}$ and $\alpha\left(p_{3}^{\prime}\right) \alpha\left(p_{1}^{\prime}\right)=t_{1}$. Similarly to the above arguments that led to inequalities (4.4)-(4.5), it follows from the inequality $|W|>6$ that either

$$
\min \left(\left|p_{2}^{\prime}\right|,\left|p_{1}^{\prime}\right|+\left|p_{3}^{\prime}\right|\right) \geq \frac{1}{3}|W|
$$

$$
\begin{aligned}
& \text { if }\left|p_{1}^{\prime}\right|+\left|p_{3}^{\prime}\right|<\left|p_{2}^{\prime}\right| \text { or } \\
& \qquad \min \left(\left|p_{2}^{\prime}\right|,\left|p_{1}^{\prime}\right|+\left|p_{3}^{\prime}\right|\right)>\min \left(\left|p_{2}\right|,\left|p_{1}\right|+\left|p_{3}\right|\right)
\end{aligned}
$$

if $\left|p_{1}^{\prime}\right|+\left|p_{3}^{\prime}\right| \geq\left|p_{2}^{\prime}\right|$. In either case, we obtain a contradiction to the maximality of the pair $v_{1}, v_{2}$.

Now assume that $\min \left(\left|p_{1}\right|,\left|p_{3}\right|\right)=0$. For definiteness, let $\left|p_{i}\right|=0, i \in\{1,3\}$. Then we can pick vertices $v_{1}^{\prime}, v_{2}^{\prime} \in P_{W}$ for which $\alpha\left(v_{1}^{\prime}\right)=\alpha\left(v_{2}^{\prime}\right), v_{1}^{\prime}<v_{2}^{\prime}$ and, if $P_{W}\left(\right.$ fact, $\left.v_{1}^{\prime}, v_{2}^{\prime}\right)=p_{1}^{\prime} p_{2}^{\prime} p_{3}^{\prime}$, then the subpaths $p_{1}^{\prime}, p_{2}^{\prime}, p_{3}^{\prime}$ of $P_{W}$ have the properties that $p_{2}^{\prime}=p_{4-i}, p_{i}^{\prime}=p_{2},\left|p_{4-i}^{\prime}\right|=\left|p_{i}\right|=0$. Hence, $\left|p_{2}^{\prime}\right|>\left|p_{1}^{\prime}\right|+\left|p_{3}^{\prime}\right|$ and

$$
\min \left(\left|p_{2}^{\prime}\right|,\left|p_{1}^{\prime}\right|+\left|p_{3}^{\prime}\right|\right)=\min \left(\left|p_{2}\right|,\left|p_{1}\right|+\left|p_{3}\right|\right) .
$$

This means that the subcase $\min \left(\left|p_{1}\right|,\left|p_{3}\right|\right)=0$ is reduced to the case $\left|p_{2}^{\prime}\right| \geq\left|p_{1}^{\prime}\right|+$ $\left|p_{3}^{\prime}\right|$ which was considered above.

The case $\ell=j=1$ is complete and it is shown that the inequality (4.6) is false, hence, for every $j=1, \ldots, \ell$, we have $\left|f_{j} t_{j} f_{j}^{-1}\right|<\frac{1}{2}|W|$.

Suppose that $\left|f_{j} t_{j} f_{j}^{-1}\right| \geq \frac{1}{3}|W|$ for some $j$. Pick vertices $v_{1}^{\prime}, v_{2}^{\prime} \in P_{W}$ so that if $P_{W}\left(\right.$ fact, $\left.v_{1}^{\prime}, v_{2}^{\prime}\right)=p_{1}^{\prime} p_{2}^{\prime} p_{3}^{\prime}$, then either $\alpha\left(p_{2}^{\prime}\right)=f_{j} t_{j} f_{j}^{-1}$ in case when $f_{j} t_{j} f_{j}^{-1}$ is a subpath of one of $q_{1}, q_{3}$, or $\alpha\left(p_{3}^{\prime}\right) \alpha\left(p_{1}^{\prime}\right)=f_{j} t_{j} f_{j}^{-1}$ in case when $f_{j} t_{j} f_{j}^{-1}$ has common edges with both $q_{1}$ and $q_{3}$. Since $\left|f_{j} t_{j} f_{j}^{-1}\right|<\frac{1}{2}|W|$, it follows that

$$
\min \left(\left|p_{2}^{\prime}\right|,\left|p_{1}^{\prime}\right|+\left|p_{3}^{\prime}\right|\right) \geq \frac{1}{3}|W| \text {. }
$$


A contradiction to the maximality of the pair $v_{1}, v_{2}$ proves that $\left|f_{j} t_{j} f_{j}^{-1}\right|<\frac{1}{3}|W|$ for every $j=1, \ldots, \ell$. Since $\left|p_{1}\right|+\left|p_{3}\right|>\frac{2}{3}|W|$, we get $\ell \geq 3$ and, for some $j \geq 2$, we obtain

$$
\frac{1}{3}|W| \leq\left|f_{1} t_{1} f_{1}^{-1} \ldots f_{j} t_{j} f_{j}^{-1}\right| \leq \frac{2}{3}|W| .
$$

This means the existence of vertices $v_{1}^{\prime}, v_{2}^{\prime} \in P_{W}$ for which $\alpha\left(v_{1}^{\prime}\right)=\alpha\left(v_{2}^{\prime}\right)$ and if $P_{W}\left(\right.$ fact, $\left.v_{1}^{\prime}, v_{2}^{\prime}\right)=p_{1}^{\prime} p_{2}^{\prime} p_{3}^{\prime}$ then the subpaths $p_{1}^{\prime}, p_{2}^{\prime}, p_{3}^{\prime}$ have the following properties: Either $\alpha\left(p_{2}^{\prime}\right)=f_{1} t_{1} f_{1}^{-1} \ldots f_{j} t_{j} f_{j}^{-1}$ or $\alpha\left(p_{3}^{\prime}\right) \alpha\left(p_{1}^{\prime}\right)=f_{1} t_{1} f_{1}^{-1} \ldots f_{j} t_{j} f_{j}^{-1}$. The it is clear that

$$
\min \left(\left|p_{2}^{\prime}\right|,\left|p_{1}^{\prime}\right|+\left|p_{3}^{\prime}\right|\right) \geq \frac{1}{3}|W| .
$$

This contradiction to the choice of the pair $v_{1}, v_{2}$ completes the second main case when $\left|p_{2}\right|<\left|p_{1}\right|+\left|p_{3}\right|$.

Lemma 4.2. Suppose $\Delta$ is a disk diagram with property (A) over presentation (1.2) and $\varphi\left(\left.\partial\right|_{0} \Delta\right) \equiv W$ with $|W|>2$. Then one of the following two claims holds.

(a) There are vertices $v_{1}, v_{2} \in P_{W}$ such that $\alpha\left(v_{1}\right)=\alpha\left(v_{2}\right), v_{1}<v_{2}$, and if $P_{W}\left(\right.$ fact $\left., v_{1}, v_{2}\right)=p_{1} p_{2} p_{3}$ is the factorization of $P_{W}$ defined by $v_{1}, v_{2}$, then

$$
\min \left(\left|p_{2}\right|,\left|p_{1}\right|+\left|p_{3}\right|\right) \geq \frac{1}{6}|W| .
$$

(b) There exists a face $\Pi$ in $\Delta$ with $|\partial \Pi| \geq 2$ and there are vertices $v_{1}, v_{2} \in P_{W}$ such that $\alpha\left(v_{1}\right), \alpha\left(v_{2}\right) \in \partial \Pi, v_{1}<v_{2}$, and if $P_{W}\left(\right.$ fact, $\left.v_{1}, v_{2}\right)=p_{1} p_{2} p_{3}$ then

$$
\min \left(\left|p_{2}\right|,\left|p_{1}\right|+\left|p_{3}\right|\right) \geq \frac{1}{6}|W| .
$$

In addition, if $(\partial \Pi)^{-1}=e_{1} \ldots e_{|\partial \Pi|}$, where $e_{i} \in \vec{\Delta}(1)$, and $\partial \Delta=e_{1} h_{1} \ldots e_{|\partial \Pi|} h_{|\partial \Pi|}$, where $h_{i}$ is a closed subpath of $\partial \Delta$, then, for every $i, h_{i}$ is a subpath of either $\alpha\left(p_{2}\right)$ or $\alpha\left(p_{3}\right) \alpha\left(p_{1}\right)$ and $\left|h_{i}\right| \leq \frac{5}{6}|W|$, see Fig. 4.2.

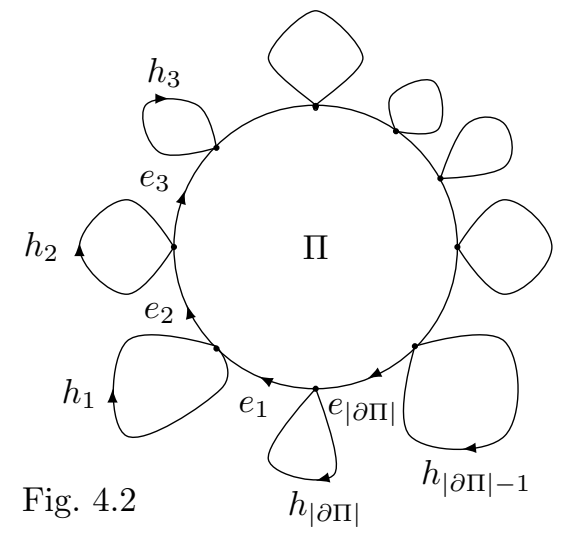

Proof. Since $\Delta$ has property (A), it follows that if $e \in \partial \Pi$, where $\Pi$ is a face of $\Delta$, then $e^{-1} \in \partial \Delta$ and if $e \in \partial \Delta$, then $e^{-1} \in \partial \Pi^{\prime} \cup \partial \Delta$, where $\Pi^{\prime}$ is a face of $\Delta$.

Consider a planar graph $\Gamma_{\Delta}$ constructed from $\Delta$ as follows. For every face $\Pi$ of $\Delta$, we pick a vertex $o_{\Pi}$ in the interior of $\Pi$. The vertex set of $\Gamma_{\Delta}$ is

$$
V\left(\Gamma_{\Delta}\right):=\Delta(0) \cup\left\{o_{\Pi} \mid \Pi \in \Delta(2)\right\},
$$

where $\Delta(i)$ is the set of $i$-cells of $\Delta, i=0,1,2$. For every face $\Pi$ of $\Delta$, we delete nonoriented edges of $\partial \Pi$ and draw $|\partial \Pi|$ nonoriented edges that connect $o_{\Pi}$ to all vertices of $\partial \Pi$, see Fig. 4.3. 


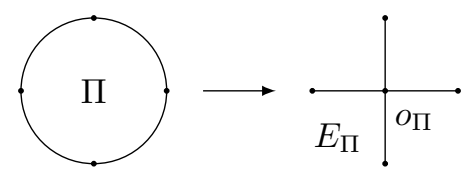

Fig. 4.3

We draw these edges so that their interiors are disjoint and are contained in the interior of $\Pi$. Let $E_{\Pi}$ denote the set of these $|\partial \Pi|$ edges. The set $E\left(\Gamma_{\Delta}\right)$ of nonoriented edges of $\Gamma_{\Delta}$ is $\Delta(1)$, without edges of faces of $\Delta$, combined with $\cup_{\Pi \in \Delta(2)} E_{\Pi}$, hence,

$$
E\left(\Gamma_{\Delta}\right):=\cup_{\Pi \in \Delta(2)} E_{\Pi} \cup(\Delta(1) \backslash\{e \mid e \in \Delta(1), e \in \partial \Pi, \Pi \in \Delta(2)\}) .
$$

It follows from definitions that $\left|V\left(\Gamma_{\Delta}\right)\right|=|\Delta(0)|+|\Delta(2)|,\left|E\left(\Gamma_{\Delta}\right)\right|=|\Delta(1)|$ and that $\Gamma_{\Delta}$ is a tree. Assigning labels to oriented edges of $\Gamma_{\Delta}$, by using letters from $\mathcal{A}^{ \pm 1}$, we can turn $\Gamma_{\Delta}$ into a disk diagram over presentation $\left\langle a_{1}, \ldots, a_{m} \| \varnothing\right\rangle$ of the free group $F(\mathcal{A})$.

Denote $\varphi\left(\left.\partial\right|_{o^{\prime}} \Gamma_{\Delta}\right) \equiv W^{\prime}$ for some vertex $o^{\prime} \in \Delta(0)$, where $\left|W^{\prime}\right|=|\vec{\Delta}(1)|$, and let $\alpha^{\prime}\left(P_{W^{\prime}}\right)=\left.\partial\right|_{o^{\prime}} \Gamma_{\Delta}=\left.\partial\right|_{0} \Gamma_{\Delta}$. Since $\left|W^{\prime}\right| \geq|W|>2$, Lemma 4.1 applies to $\Gamma_{\Delta}$ and yields the existence of vertices $u_{1}, u_{2} \in P_{W^{\prime}}$ such that $\alpha^{\prime}\left(u_{1}\right)=\alpha^{\prime}\left(u_{2}\right)$ in $\partial \Gamma_{\Delta}$, $u_{1}<u_{2}$, and if $P_{W^{\prime}}\left(\right.$ fact, $\left.u_{1}, u_{2}\right)=r_{1} r_{2} r_{3}$, then

$$
\min \left(\left|r_{2}\right|,\left|r_{1}\right|+\left|r_{3}\right|\right) \geq \frac{1}{3}\left|W^{\prime}\right| \text {. }
$$

First suppose that $\alpha^{\prime}\left(u_{1}\right)$ is a vertex of $\Delta$. It follows from the definition of the tree $\Gamma_{\Delta}$ that there is a factorization $P_{W}=p_{1} p_{2} p_{3}$ of the path $P_{W}$ such that the vertex $\alpha\left(\left(p_{2}\right)_{-}\right)=\alpha\left(\left(p_{2}\right)_{+}\right)$is $\alpha^{\prime}\left(u_{1}\right) \in \Delta(0)$ and $\left|p_{i}\right| \leq\left|r_{i}\right| \leq 2\left|p_{i}\right|, i=1,2,3$. Indeed, to get from $\partial \Delta$ to $\partial \Gamma_{\Delta}$ we replace every edge $e \in \partial \Pi$, $\Pi \in \Delta(2)$, by two edges of $E_{\Pi}$, see Fig. 4.3. Hence, if $r$ is a subpath of $\partial \Gamma_{\Delta}$ and $p$ is a corresponding to $r$ subpath of $\partial \Delta$ with $r_{-}=p_{-} \in \Delta(0), r_{+}=p_{+} \in \Delta(0)$, then $|p| \leq|r| \leq 2|p|$. Then it follows from (4.8) that

$$
\min \left(\left|p_{2}\right|,\left|p_{1}\right|+\left|p_{3}\right|\right) \geq \frac{1}{2} \min \left(\left|r_{2}\right|,\left|r_{1}\right|+\left|r_{3}\right|\right) \geq \frac{1}{6}\left|W^{\prime}\right| \geq \frac{1}{6}|W|
$$

as required.

Now assume that $\alpha^{\prime}\left(u_{1}\right)=\alpha^{\prime}\left(u_{2}\right)=o_{\Pi}$ for some face $\Pi \in \Delta(2)$. Let $e_{1}, \ldots, e_{k}$, $f_{1}, \ldots, f_{\ell}, k, \ell \geq 0$, be all oriented edges of $\Gamma_{\Delta}$ that start at the vertex $\alpha^{\prime}\left(u_{1}\right)=$ $\alpha^{\prime}\left(u_{2}\right)=o_{\Pi}$ so that

$$
\alpha^{\prime}\left(r_{2}\right)=e_{1} s_{1} e_{1}^{-1} \ldots e_{k} s_{k} e_{k}^{-1}, \quad \alpha^{\prime}\left(r_{3}\right) \alpha^{\prime}\left(r_{1}\right)=f_{1} t_{1} f_{1}^{-1} \ldots f_{\ell} t_{\ell} f_{\ell}^{-1},
$$

where $s_{1}, \ldots, s_{k}$ and $t_{1}, \ldots, t_{\ell}$ are closed subpaths of $\alpha^{\prime}\left(r_{2}\right)$ and $\alpha^{\prime}\left(r_{3}\right) \alpha^{\prime}\left(r_{1}\right)$, resp. Since $\alpha^{\prime}\left(u_{1}\right)=\alpha^{\prime}\left(u_{2}\right)=o_{\Pi}$, it follows that $k+\ell=|\partial \Pi|$. Since $\min \left(\left|r_{2}\right|,\left|r_{1}\right|+\left|r_{3}\right|\right) \geq$ $\frac{1}{3}\left|W^{\prime}\right|>0$ is an integer, we also have that $k, \ell \geq 1$ and $|\partial \Pi|>1$. If $\left|r_{3}\right|>0$, we consider vertices $u_{1}^{\prime}:=u_{1}+1, u_{2}^{\prime}:=u_{2}+1$. On the other hand, if $\left|r_{3}\right|=0$, then $\left|r_{1}\right|>0$ and we consider vertices $u_{1}^{\prime}:=u_{1}-1, u_{2}^{\prime}:=u_{2}-1$. In either case, denote $P_{W^{\prime}}\left(u_{1}^{\prime}, u_{2}^{\prime}\right)=r_{1}^{\prime} r_{2}^{\prime} r_{3}^{\prime}$. Then $\left|r_{2}^{\prime}\right|=\left|r_{2}\right|$ and $\left|r_{1}^{\prime}\right|+\left|r_{3}^{\prime}\right|=\left|r_{1}\right|+\left|r_{3}\right|$, hence, by virtue of (4.8),

$$
\min \left(\left|r_{2}^{\prime}\right|,\left|r_{1}^{\prime}\right|+\left|r_{3}^{\prime}\right|\right) \geq \frac{1}{3}\left|W^{\prime}\right| \text {. }
$$

Note that the vertices $\alpha^{\prime}\left(\left(r_{2}^{\prime}\right)_{-}\right), \alpha^{\prime}\left(\left(r_{2}^{\prime}\right)_{+}\right)$belong to the boundary $\partial \Pi$. Hence, as above, there is also a factorization $P_{W}\left(\right.$ fact, $\left.v_{1}, v_{2}\right)=p_{1} p_{2} p_{3}$ such that $\alpha\left(v_{1}\right)=$ $\alpha^{\prime}\left(u_{1}^{\prime}\right), \alpha\left(v_{2}\right)=\alpha^{\prime}\left(u_{2}^{\prime}\right)$ and $\left|p_{i}\right| \leq\left|r_{i}^{\prime}\right| \leq 2\left|p_{i}\right|, i=1,2,3$. Therefore,

$$
\min \left(\left|p_{2}\right|,\left|p_{1}\right|+\left|p_{3}\right|\right) \geq \frac{1}{2} \min \left(\left|r_{2}^{\prime}\right|,\left|r_{1}^{\prime}\right|+\left|r_{3}^{\prime}\right|\right) \geq \frac{1}{6}\left|W^{\prime}\right| \geq \frac{1}{6}|W|
$$


as required.

It remains to observe that it follows from the definition of vertices $v_{1}, v_{2}$ that every $h_{i}$ is a subpath of one of the paths $\alpha\left(p_{2}\right), \alpha\left(p_{3}\right) \alpha\left(p_{1}\right)$. In particular, $\left|h_{i}\right| \leq$ $\frac{5}{6}|W|$, as desired.

In the definitions below, we assume that $\Delta$ is a disk diagram over presentation (1.2) such that $\Delta$ has property $(\mathrm{A}), \varphi\left(\left.\partial\right|_{0} \Delta\right) \equiv W,|W|>0$, and that the pair $(W, \Delta)$ is fixed.

\section{A 6-tuple}

$$
b=(b(1), b(2), b(3), b(4), b(5), b(6))
$$

of integers $b(1), b(2), b(3), b(4), b(5), b(6)$ is called a bracket for the pair $(W, \Delta)$ if $b(1), b(2)$ satisfy the inequalities $0 \leq b(1) \leq b(2) \leq|W|$ and, in the notation $P_{W}($ fact $, b(1), b(2))=p_{1} p_{2} p_{3}$, one of the following two properties (B1)-(B2) holds true.

(B1) $b(3)=b(4)=b(5)=0, \alpha(b(1))=\alpha(b(2))$, and the disk subdiagram $\Delta_{b}$ of $\Delta$, defined by $\left.\partial\right|_{b(1)} \Delta_{b}=\alpha\left(p_{2}\right)$, contains $b(6)$ faces, see Fig. 4.4(B1).

(B2) $b(3)>0$ and $\alpha(b(1)), \alpha(b(2)) \in \partial \Pi$, where $\Pi$ is a face of $\Delta$ such that $\varphi(\partial \Pi) \equiv a_{b(3)}^{\varepsilon b(4)}, b(4)>0, \varepsilon= \pm 1$, and if $\Delta_{b}$ is the disk subdiagram of $\Delta$, defined by $\left.\partial\right|_{b(1)} \Delta_{b}=\alpha\left(p_{2}\right) u$, where $u$ is the subpath of $\partial \Pi$ with $u_{-}=\alpha(b(2))$ and $u_{+}=\alpha(b(1))$, then $\varphi(u) \equiv a_{b(3)}^{-b(5)}$ and $\left|\Delta_{b}(2)\right|=b(6)$, see Fig. 4.4(B2).

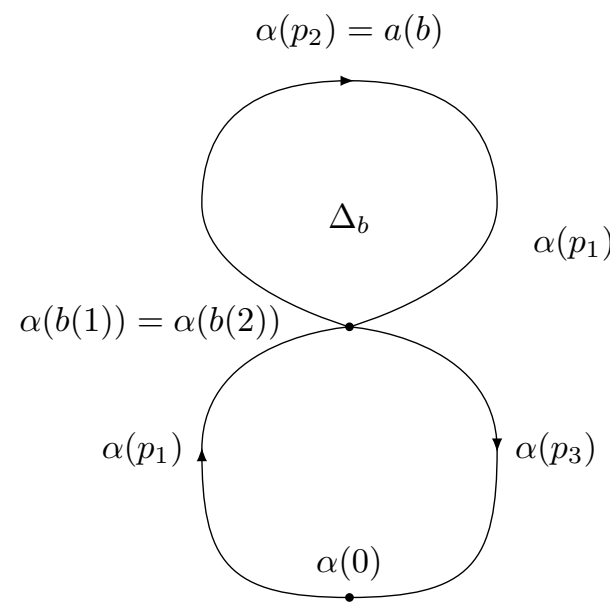

Fig. 4.4(B1)

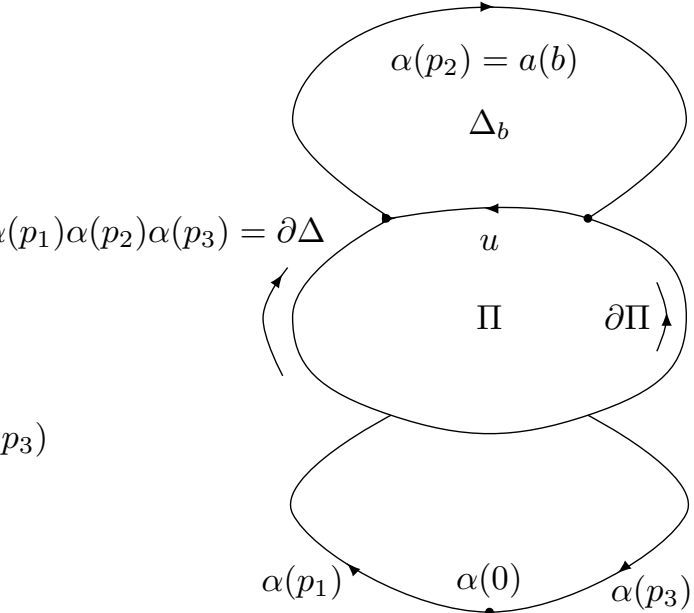

Fig. 4.4(B2)

A bracket $b$ is said to have type B1 or type B2 if the property (B1) or (B2), resp., holds for $b$. Note that the equality $b(4)=0$ in property (B1) and the inequality $b(4)>0$ in property (B2) imply that the type of a bracket is unique.

The boundary subpath $\alpha\left(p_{2}\right)$ of the disk subdiagram $\Delta_{b}$ associated with a bracket $b$ is denoted $a(b)$ and is called the arc of $b$, see Figs. 4.4(B1)-4.4(B2).

For example, $b_{v}=(v, v, 0,0,0,0)$ is a bracket of type B1 for every vertex $v$ of $P_{W}$, called a starting bracket at $v$. Note that $a\left(b_{v}\right)=\alpha(v)=\alpha\left(b_{v}(1)\right)$. 
The final bracket for $(W, \Delta)$ is $b_{F}=(0,|W|, 0,0,0,|\Delta(2)|)$, it has type B1 and $a\left(b_{F}\right)=\left.\partial\right|_{0} \Delta$.

Let $B$ be a set of brackets for the pair $(W, \Delta)$, perhaps, $B$ is empty, $B=\varnothing$. We say that $B$ is a bracket system if, for every pair $b, c \in B$ of distinct brackets, either $b(2) \leq c(1)$ or $c(2) \leq b(1)$. In particular, the arcs of distinct brackets in $B$ have no edges in common. A bracket system consisting of a single final bracket is called a final bracket system.

Now we describe four kinds of operations over brackets and over bracket systems: additions, extensions, turns, and mergers. Let $B$ be a bracket system.

Additions.

Suppose $b$ is a starting bracket, $b \notin B$, and $B \cup\{b\}$ is a bracket system. Then we may add $b$ to $B$ thus making an addition operation over $B$.

Extensions.

Suppose $b \in B, b=(b(1), b(2), b(3), b(4), b(5), b(6))$, and $e_{1} a(b) e_{2}$ is a subpath of $\left.\partial\right|_{0} \Delta$, where $a(b)$ is the arc of $b$ and $e_{1}, e_{2}$ are edges one of which could be missing.

Assume that $b$ is of type B2, in particular, $b(3), b(4)>0$. Using the notation of the condition (B2), suppose $e_{1}^{-1}$ is an edge of $\partial \Pi$, and $\varphi\left(e_{1}\right)=a_{b(3)}^{\varepsilon_{1}}$, where $\varepsilon_{1}= \pm 1$.

If $|b(5)| \leq b(4)-2$ and $\varepsilon_{1} b(5) \geq 0$, then we consider a bracket $b^{\prime}$ such that

$$
\begin{aligned}
& b^{\prime}(1)=b(1)-1, b^{\prime}(2)=b(2), b^{\prime}(3)=b(3), \\
& b^{\prime}(4)=b(4), b^{\prime}(5)=b(5)+\varepsilon_{1}, b^{\prime}(6)=b(6) .
\end{aligned}
$$

Note that $a\left(b^{\prime}\right)=e_{1} a(b)$. We say that $b^{\prime}$ is obtained from $b$ by an extension of type 1 (on the left). If $(B \backslash\{b\}) \cup\left\{b^{\prime}\right\}$ is a bracket system, then replacement of $b \in B$ with $b^{\prime}$ in $B$ is called an extension operation over $B$ of type 1 .

On the other hand, if $|b(5)|=b(4)-1$ and $\varepsilon_{1} b(5) \geq 0$, then we consider a bracket $b^{\prime}$ such that

$$
b^{\prime}(1)=b(1)-1, b^{\prime}(2)=b(2), b^{\prime}(3)=b^{\prime}(4)=b^{\prime}(5)=0, b^{\prime}(6)=b(6)+1 .
$$

In this case, we say that $b^{\prime}$ is obtained from $b$ by an extension of type 2 (on the left). Note that $a\left(b^{\prime}\right)=e_{1} a(b)$ and $b^{\prime}$ has type B1. If $(B \backslash\{b\}) \cup\left\{b^{\prime}\right\}$ is a bracket system, then replacement of $b \in B$ with $b^{\prime}$ in $B$ is called an extension operation over $B$ of type 2 .

Analogously, assume that $b$ has type $\mathrm{B} 2, e_{2}^{-1}$ is an edge of $\partial \Pi$, and $\varphi\left(e_{2}\right)=a_{b(3)}^{\varepsilon_{2}}$, where $\varepsilon_{2}= \pm 1$.

If $|b(5)| \leq b(4)-2$ and $\varepsilon_{2} b(5) \geq 0$, then we consider a bracket $b^{\prime}$ such that

$$
\begin{aligned}
& b^{\prime}(1)=b(1), b^{\prime}(2)=b(2)+1, b^{\prime}(3)=b(3), \\
& b^{\prime}(4)=b(4), b^{\prime}(5)=b(5)+\varepsilon_{2}, b^{\prime}(6)=b(6) .
\end{aligned}
$$

Note that $a\left(b^{\prime}\right)=a(b) e_{2}$ and $b^{\prime}$ has type B2. We say that $b^{\prime}$ is obtained from $b$ by an extension of type 1 (on the right). If $(B \backslash\{b\}) \cup\left\{b^{\prime}\right\}$ is a bracket system, then replacement of $b \in B$ with $b^{\prime}$ in $B$ is called an extension operation over $B$ of type 1 .

On the other hand, if $|b(5)|=b(4)-1$, then we may consider a bracket $b^{\prime}$ such that

$$
b^{\prime}(1)=b(1), b^{\prime}(2)=b(2)+1, b^{\prime}(3)=b^{\prime}(4)=b^{\prime}(5)=0, b^{\prime}(6)=b(6)+1 .
$$

Note that $a\left(b^{\prime}\right)=a(b) e_{2}$ and $b^{\prime}$ has type B1. We say that $b^{\prime}$ is obtained from $b$ by an extension of type 2 (on the right). If $(B \backslash\{b\}) \cup\left\{b^{\prime}\right\}$ is a bracket system, 
then replacement of $b \in B$ with $b^{\prime}$ in $B$ is called an extension operation over $B$ of type 2 .

Assume that $b \in B$ is a bracket of type $\mathrm{B} 1, e_{1} a(b) e_{2}$ is a subpath of $\left.\partial\right|_{0} \Delta$, both $e_{1}, e_{2}$ exist, and $e_{1}=e_{2}^{-1}$. Consider a bracket $b^{\prime}$ of type B1 such that

$$
b^{\prime}(1)=b(1)-1, b^{\prime}(2)=b(2)+1, b^{\prime}(3)=b^{\prime}(4)=b^{\prime}(5)=0, b^{\prime}(6)=b(6) .
$$

Note that $a\left(b^{\prime}\right)=e_{1} a(b) e_{2}$. We say that $b^{\prime}$ is obtained from $b$ by an extension of type 3. If $(B \backslash\{b\}) \cup\left\{b^{\prime}\right\}$ is a bracket system, then replacement of $b \in B$ with $b^{\prime}$ in $B$ is called an extension operation over $B$ of type 3 .

Turns.

Let $b \in B$ be a bracket of type B1. Then $b(3)=b(4)=b(5)=0$. Suppose $\Pi$ is a face in $\Delta$ such that $\Pi$ is not in the disk subdiagram $\Delta_{b}$, associated with $b$ and bounded by the arc $a(b)$ of $b$, and $\alpha(b(1)) \in \partial \Pi$. If $\varphi(\partial \Pi)=a_{j}^{\varepsilon n_{\Pi}}$, where $\varepsilon= \pm 1, n_{\Pi} \in E_{j}$, then we consider a bracket $b^{\prime}$ with $b^{\prime}(i)=b(i)$ for $i=1,2,5,6$, and $b^{\prime}(3)=j, b^{\prime}(4)=n_{\Pi}$. Note that $b^{\prime}$ has type B2. We say that $b^{\prime}$ is obtained from $b$ by a turn operation. Replacement of $b \in B$ with $b^{\prime}$ in $B$ is also called a turn operation over $B$. Note that $(B \backslash\{b\}) \cup\left\{b^{\prime}\right\}$ is automatically a bracket system (because so is $B$ ).

\section{Mergers.}

Now suppose that $b, c \in B$ are distinct brackets such that $b(2)=c(1)$ and one of $b(3), c(3)$ is 0 . Then one of $b, c$ is of type B1 and the other has type B1 or B2. Consider a bracket $b^{\prime}$ such that $b^{\prime}(1)=b(1), b^{\prime}(2)=c(2)$, and $b^{\prime}(i)=b(i)+c(i)$ for $i=3,4,5,6$. Note that $a\left(b^{\prime}\right)=a\left(b_{1}\right) a\left(b_{2}\right)$ and $b^{\prime}$ is of type B1 if both $b_{i}, b_{j}$ have type $\mathrm{B} 1$ or $b^{\prime}$ is of type $\mathrm{B} 2$ if one of $b, c$ has type $\mathrm{B} 2$. We say that $b^{\prime}$ is obtained from $b, c$ by a merger operation. Taking both $b, c$ out of $B$ and putting $b^{\prime}$ in $B$ is a merger operation over $B$. Note that $(B \backslash\{b, c\}) \cup\left\{b^{\prime}\right\}$ is automatically a bracket system.

We will say that additions, extensions, turns and mergers, as defined above, are elementary operations over brackets and bracket systems for the pair $(W, \Delta)$.

Assume that one bracket system $B_{\ell}$ is obtained from another bracket system $B_{0}$ by a finite sequence $\Omega$ of elementary operations and $B_{0}, B_{1}, \ldots, B_{\ell}$ is the corresponding to $\Omega$ sequence of bracket systems. Such a sequence $B_{0}, B_{1}, \ldots, B_{\ell}$ of bracket systems will be called operational.

We will say that the sequence $B_{0}, B_{1}, \ldots, B_{\ell}$ has size bounded by $\left(k_{1}, k_{2}\right)$ if $\ell \leq k_{1}$ and, for every $i$, the number of brackets in $B_{i}$ is at most $k_{2}$. Whenever it is not ambiguous, we will also say that $\Omega$ has size bounded by $\left(k_{1}, k_{2}\right)$ if so does the corresponding to $\Omega$ sequence $B_{0}, B_{1}, \ldots, B_{\ell}$ of bracket systems.

Lemma 4.3. There exists a sequence of elementary operations that converts the empty bracket system for $(W, \Delta)$ into the final bracket system and has size bounded by $(8|W|,|W|+1)$.

Proof. For every $k$ with $0 \leq k \leq|W|$, consider a starting bracket $(k, k, 0,0,0,0)$ for $(W, \Delta)$. Making $|W|+1$ additions, we get a bracket system

$$
B_{W}=\{(k, k, 0,0,0,0)|0 \leq k \leq| W \mid\}
$$

of $|W|+1$ starting brackets. Now, looking at the disk diagram $\Delta$, we can easily find a sequence of extensions, turns and mergers that converts $B_{W}$ into the final bracket system, denoted $B_{\ell}$. Note that the inequality $b(4) \leq|W|$ for every bracket 
$b$ of intermediate systems follows from definitions and Lemma 2.2. To estimate the total number of elementary operations, we note that the number of additions is $|W|+1$. The number of extensions is at most $|W|$ because every extension applied to a bracket system $B$ increases the number

$$
\eta(B):=\sum_{b \in B}(b(2)-b(1))
$$

by 1 or 2 and $\eta\left(B_{W}\right)=0$, whereas $\eta\left(B_{\ell}\right)=|W|$. The number of mergers is $|W|$ because the number of brackets $|B|$ in a bracket system $B$ decreases by 1 if $B$ is obtained by a merger and $\left|B_{W}\right|=|W|+1,\left|B_{\ell}\right|=1$. The number of turns does not exceed the total number of additions, extensions, and mergers, because a turn operation is applied to a bracket of type B1 and results in a bracket of type B2 to which a turn operation may not be applied, whence a turn operation succeeds an addition, or an extension, or a merger. Therefore, the number of turns is at most $3|W|+1$ and so $\ell \leq 6|W|+2 \leq 8|W|$.

Lemma 4.4. Suppose there is a sequence $\Omega$ of elementary operations that converts the empty bracket system $E$ for $(W, \Delta)$ into the final bracket system $F$ and has size bounded by $\left(k_{1}, k_{2}\right)$. Then there is also a sequence of elementary operations that transforms $E$ into $F$ and has size bounded by $\left(11|W|, k_{2}\right)$.

Proof. Assume that the sequence $\Omega$ has an addition operation which introduces a starting bracket $c=(k, k, 0,0,0,0)$ with $0 \leq k \leq|W|$. Since the final bracket system contains no starting brackets, $c$ must disappear and an elementary operation is applied to $c$. If a merger is applied to $c$ and another bracket $b$ and the merger yields $\widehat{c}$, then $\widehat{c}=b$. This means that the addition of $c$ and the merger could be skipped without affecting the sequence otherwise. Note that the size of the new sequence $\Omega^{\prime}$ is bounded by $\left(k_{1}-2, k_{2}\right)$. Therefore, we may assume that no merger is applied to a starting bracket in $\Omega$.

Now suppose that a turn is applied to $c$, yields $c^{\prime}$ and then a merger is applied to $c^{\prime}, b$ and the merger produces $\widehat{c}$. Note that $c^{\prime}$ has type B2 and $b$ has type B1. Then it is possible to apply a turn to $b$ and get $b^{\prime}$ with $b^{\prime}=\widehat{c}$. Hence, we can skip the addition of $c$, the turn of $c$, the merger and use, in their place, a turn of $b$. Clearly, the size of the new sequence $\Omega^{\prime}$ is bounded by $\left(k_{1}-2, k_{2}\right)$.

Thus, by induction on $k_{1}$, we may assume that, for every starting bracket $c$ which is added by $\Omega$, an extension is applied to $c$ or an extension is applied to $c^{\prime}$ and $c^{\prime}$ is obtained from $c$ by a turn.

Now we will show that, for every starting bracket $c$ which is added by $\Omega$, there are at most 2 operations of additions of $c$ in $\Omega$. Arguing on the contrary, assume that $c_{1}, c_{2}, c_{3}$ are the brackets equal to $c$ whose additions are done in $\Omega$. By the above remark, for every $i=1,2,3$, either an extension is applied to $c_{i}$, resulting in a bracket $\widehat{c}_{i}$, or a turn is applied to $c_{i}$, resulting in $c_{i}^{\prime}$, and then an extension is applied to $c_{i}^{\prime}$, resulting in a bracket $\widehat{c}_{i}$.

Let $c_{1}, c_{2}, c_{3}$ be listed in the order in which the brackets $\widehat{c}_{1}, \widehat{c}_{2}, \widehat{c}_{3}$ are created by $\Omega$. Note that if $\widehat{c}_{1}$ is obtained from $c_{1}$ by an extension (with no turn), then the extension has type 3 and

$$
\widehat{c}_{1}(1)=c_{1}(1)-1, \widehat{c}_{1}(2)=c_{1}(2)+1 .
$$

This means that brackets $\widehat{c}_{2}, \widehat{c}_{3}$ could not be created by an extension after $\widehat{c}_{1}$ appears, as $d(2) \leq d^{\prime}(1)$ or $d(1) \geq d^{\prime}(2)$ for distinct brackets $d, d^{\prime} \in B$ of any 
bracket system $B$. This contradiction proves that $\widehat{c}_{1}$ is obtained from $c_{1}^{\prime}$ by an extension. Then $c_{1}^{\prime}$ has type B2, the extension has type 1 or 2 (and is either on the left or on the right). Similarly to the forgoing argument, we can see that if $\widehat{c}_{1}$ is obtained by an extension on the left/right, then $\widehat{c}_{2}$ must be obtained by an extension on the right/left, resp., and that $\widehat{c}_{3}$ cannot be obtained by any extension. This contradiction proves that it is not possible to have in $\Omega$ more than two additions of any starting bracket $c$. Thus, the number of additions in $\Omega$ is at most $2|W|+2$.

As in the proof of Lemma 4.3, the number of extensions is at most $|W|$ and the number of mergers is $\leq 2|W|+1$. Hence, the number of turns is $\leq 5|W|+3$ and the total number of elementary operations is at most $10|W|+6 \leq 11|W|$ as desired.

Lemma 4.5. Let there be a sequence $\Omega$ of elementary operations that transforms the empty bracket system for the pair $(W, \Delta)$ into the final bracket system and has size bounded by $\left(k_{1}, k_{2}\right)$ and let $c$ be a starting bracket for $(W, \Delta)$. Then there is also a sequence of elementary operations that converts the bracket system $\{c\}$ into the final bracket system and has size bounded by $\left(k_{1}+1, k_{2}+1\right)$.

Proof. Let

$$
B_{0}, B_{1}, \ldots, B_{\ell}
$$

be the corresponding to $\Omega$ sequence of bracket systems, where $B_{0}$ is empty and $B_{\ell}$ is final.

First suppose that $c(1)=0$ or $c(1)=|W|$.

Assume that no addition of $c$ is used in $\Omega$. Setting $B_{i}^{\prime}:=B_{i} \cup\{c\}, i=0,1, \ldots, \ell$, we obtain an operational sequence of bracket systems $B_{0}^{\prime}, \ldots, B_{\ell}^{\prime}$ that starts with $\{c\}$ and ends in the bracket system $B_{\ell}^{\prime}=\left\{c, d_{F}\right\}$, where $d_{F}$ is the final bracket for $(W, \Delta)$. Note that $B_{i+1}^{\prime}$ can be obtained from $B_{i}^{\prime}$ by the same elementary operation that was used to get $B_{i+1}$ from $B_{i}$. A merger operation applied to $B_{\ell}^{\prime}$ yields the final bracket system $B_{\ell+1}^{\prime}=\left\{d_{F}\right\}$ and $B_{0}^{\prime}, \ldots, B_{\ell}^{\prime}, B_{\ell+1}^{\prime}$ is a desired sequence of bracket systems of size bounded by $\left(k_{1}+1, k_{2}+1\right)$.

Now suppose that an addition of $c$ is used in $\Omega, B_{i^{*}+1}=B_{i^{*}} \cup\{c\}$ is obtained from $B_{i^{\prime}}$ by addition of $c$, and $i^{*}$ is minimal with this property. Define $B_{j}^{\prime}:=B_{j} \cup\{c\}$ for $j=0, \ldots, i^{*}$ and $B_{i^{*}+1}^{\prime}:=B_{i^{*}+2}, \ldots, B_{\ell-1}^{\prime}:=B_{\ell}$. Then $B_{0}^{\prime}, \ldots, B_{\ell-1}^{\prime}$ is a desired operational sequence of bracket systems that starts with $\{c\}$, ends in the final bracket system, and has size bounded by $\left(k_{1}-1, k_{2}+1\right)$.

We may now assume that $0<c(1)<|W|$. Let $B_{i^{*}}$ be the first bracket system of the sequence (4.9) such that $B_{i^{*}} \cup\{c\}$ is not a bracket system. The existence of such $B_{i^{*}}$ follows from the facts that $B_{0} \cup\{c\}$ is a bracket system and $B_{\ell} \cup\{c\}$ is not. Since $B_{0} \cup\{c\}$ is a bracket system, it follows that $i^{*} \geq 1$ and $B_{i^{*}-1} \cup\{c\}$ is a bracket system. Since $B_{i^{*}-1} \cup\{c\}$ is a bracket system and $B_{i^{*}} \cup\{c\}$ is not, there is a bracket $b \in B_{i^{*}}$ such that $b(1)<c(1)<b(2)$ and $b$ is obtained from a bracket $d_{1} \in B_{i^{*}-1}$ by an extension or $b$ is obtained from brackets $d_{1}, d_{2} \in B_{i^{*}-1}$ by a merger. In either case, it follows from definitions of elementary operations that $d_{i}(j)=c(1)$ for some $i, j \in\{1,2\}$. Hence, we can use a merger applied to $d_{i}(j)$ and $c$ which would result in $d_{i}(j)$, i.e., in elimination of $c$ from $B_{i^{*}-1} \cup\{c\}$ and in getting thereby $B_{i^{*}-1}$ from $B_{i^{*}-1} \cup\{c\}$. Now we can see that the original sequence of elementary operations, together with the merger $B_{i^{*}-1} \cup\{c\} \rightarrow B_{i^{*}-1}$ can be used to produce the following operational sequence of bracket systems

$$
B_{0} \cup\{c\}, \ldots, B_{i^{*}-1} \cup\{c\}, B_{i^{*}-1}, B_{i^{*}}, \ldots, B_{\ell} .
$$

Clearly, the size of this new sequence is bounded by $\left(k_{1}+1, k_{2}+1\right)$, as required. 
Lemma 4.6. There exists a sequence of elementary operations that converts the empty bracket system for the pair $(W, \Delta)$ into the final bracket system and has size bounded by

where $C=\left(\log \frac{6}{5}\right)^{-1}$.

$$
(11|W|, C(\log |W|+1))
$$

Proof. First suppose that $|W| \leq 2$. Then $\Delta$ consists of a single edge, or of a single face $\Pi$ with $|\partial \Pi| \leq 2$, or of two faces $\Pi_{1}, \Pi_{2}$ with $\left|\partial \Pi_{1}\right|=\left|\partial \Pi_{2}\right|=1$. In each of these three cases, we can easily find a sequence of elementary operations that transforms the empty bracket system for $(W, \Delta)$ into the final bracket system by using a single addition, at most two turns, and at most two extensions. Hence, the size of the sequence is bounded by

$$
(5,1) \leq(11|W|, C(\log |W|+1)
$$

as $C>1$.

Assuming $|W|>2$, we proceed by induction on the length $|W|$. By Lemma 4.2 applied to $(W, \Delta)$, we obtain either the existence of vertices $v_{1}, v_{2} \in P_{W}$ such that $v_{1}<v_{2}, \alpha\left(v_{1}\right)=\alpha\left(v_{2}\right)$ and, if $P_{W}\left(\right.$ fact $\left., v_{1}, v_{2}\right)=p_{1} p_{2} p_{3}$, then

$$
\min \left(\left|p_{2}\right|,\left|p_{1}\right|+\left|p_{3}\right|\right) \geq \frac{1}{6}|W|
$$

or we get the existence of a face $\Pi$ in $\Delta$ and vertices $v_{1}, v_{2} \in P_{W}$ with the properties stated in part (b) of Lemma 4.2 .

First assume that Lemma 4.2 (a) holds true and $\left.\partial\right|_{0} \Delta=q_{1} q_{2} q_{3}$, where $q_{i}=\alpha\left(p_{i}\right)$, $i=1,2,3$. Consider disk subdiagrams $\Delta_{1}, \Delta_{2}$ of $\Delta$ given by $\left.\partial\right|_{v_{2}} \Delta_{2}=q_{2},\left.\partial\right|_{0} \Delta_{1}=$ $q_{1} q_{3}$. Denote $W_{2} \equiv \varphi\left(q_{2}\right), W_{1} \equiv \varphi\left(q_{1}\right) \varphi\left(q_{3}\right)$ and let $P_{W_{i}}=P_{W_{i}}\left(W_{i}, \Delta_{i}\right), i=1,2$, denote the corresponding paths such that $\alpha_{1}\left(P_{W_{1}}\right)=q_{1} q_{3}$ and $\alpha_{2}\left(P_{W_{2}}\right)=q_{2}$.

Since $\left|W_{1}\right|,\left|W_{2}\right|<|W|$, it follows from the induction hypothesis that there is a sequence $\Omega_{2}$ of elementary operations for $\left(W_{2}, \Delta_{2}\right)$ that transforms the empty bracket system into the final system and has size bounded by

$$
\left(11\left|W_{2}\right|, C\left(\log \left|W_{2}\right|+1\right)\right) \text {. }
$$

Let $B_{2,0}, \ldots, B_{2, \ell_{2}}$ denote the corresponding to $\Omega_{2}$ sequence of bracket systems, where $B_{2,0}$ is empty and $B_{2, \ell_{2}}$ is final.

We also consider a sequence $\Omega_{1}$ of elementary operations for $\left(W_{1}, \Delta_{1}\right)$ that transforms the bracket system $\left\{c_{0}\right\}$, where

$$
c_{0}:=\left(\left|p_{1}\right|,\left|p_{1}\right|, 0,0,0,0\right)
$$

is a starting bracket, into the final bracket system. It follows from the induction hypothesis and Lemma 4.5 that there is such a sequence $\Omega_{1}$ of size bounded by

$$
\left(11\left|W_{1}\right|+1, C\left(\log \left|W_{1}\right|+1\right)+1\right) .
$$

Let $B_{1,0}, \ldots, B_{1, \ell_{1}}$ denote the corresponding to $\Omega_{1}$ sequence of bracket systems, where $B_{1,0}=\left\{c_{0}\right\}$ and $B_{1, \ell_{1}}$ is final.

We will show below that these two sequences $\Omega_{2}, \Omega_{1}$ of elementary operations, the first one for $\left(W_{2}, \Delta_{2}\right)$ and the second one for $\left(W_{1}, \Delta_{1}\right)$, could be modified and combined into a single sequence of elementary operations for $(W, \Delta)$ that transforms the empty bracket system into the final system and has size with the desired upper bound. 
First we observe that every bracket $b=(b(1), \ldots, b(6))$ for $\left(W_{2}, \Delta_{2}\right)$ naturally gives rise to a bracket $\widehat{b}=(\widehat{b}(1), \ldots, \widehat{b}(6))$ for $(W, \Delta)$. Specifically, define

$$
\widehat{b}:=\left(b(1)+\left|p_{1}\right|, b(2)+\left|p_{1}\right|, b(3), b(4), b(5), b(6)\right) .
$$

Let $\widehat{B}_{2, j}$ denote the bracket system obtained from $B_{2, j}$ by replacing every bracket $b \in B_{2, j}$ with $\widehat{b}$. Then $\widehat{B}_{2,0}, \ldots, \widehat{B}_{2, \ell_{2}}$ is a sequence of bracket systems for $(W, \Delta)$ that changes the empty bracket system into

$$
\widehat{B}_{2, \ell_{2}}=\left\{\left(\left|p_{1}\right|,\left|p_{1}\right|+\left|p_{2}\right|, 0,0,\left|\Delta_{2}(2)\right|\right)\right\} .
$$

Define a relation $\succeq$ on the set of pairs $(b, i)$, where $b \in B_{1, i}$, that is the reflexive and transitive closure of the relation $(c, i+1) \succ(b, i)$, where $c \in B_{1, i+1}$ is obtained from $b, b^{\prime} \in B_{1, i}$ by an elementary operation $\sigma$, where $b^{\prime}$ could be missing. It follows from the definitions that $\succeq$ is a partial order on the set of such pairs $(b, i)$ and that if $\left(b_{2}, i_{2}\right) \succeq\left(b_{1}, i_{1}\right)$ then $i_{2} \geq i_{1}$ and

$$
b_{2}(1) \leq b_{1}(1) \leq b_{1}(2) \leq b_{2}(2) .
$$

Note that the converse need not hold, e.g., if $b_{1}$ is a starting bracket and $i_{1}=i_{2}$, $b_{1}(1)=b_{1}(2)=b_{2}(1), b_{1} \neq b_{2}$, then the above inequalities hold but $\left(b_{2}, i_{2}\right) \nsucceq$ $\left(b_{1}, i_{1}\right)$.

Now we observe that every bracket $d=(d(1), \ldots, d(6)), d \in B_{1, i}$, for $\left(W_{1}, \Delta_{1}\right)$ naturally gives rise to a bracket

$$
\widehat{d}=(\widehat{d}(1), \ldots, \widehat{d}(6))
$$

for $(W, \Delta)$ in the following fashion.

If $(d, i)$ is not comparable with $\left(c_{0}, 0\right)$, where $c_{0}$ is defined in (4.12), by the relation $\succeq$ and $d(1) \leq d(2) \leq\left|p_{1}\right|$, then

$$
\widehat{d}:=d \text {. }
$$

If $(d, i)$ is not comparable with $\left(c_{0}, 0\right)$ by the relation $\succeq$ and $\left|p_{1}\right| \leq d(1) \leq d(2)$, then

$$
\begin{aligned}
& \widehat{d}:=\left(d(1)+\left|p_{2}\right|, d(2)+\left|p_{2}\right|, d(3), d(4), d(5), d(6)\right) . \\
& \text { If }(d, i) \succeq\left(c_{0}, 0\right) \text {, then } \\
& \widehat{d}:=\left(d(1), d(2)+\left|p_{2}\right|, d(3), d(4), d(5), d(6)+\left|\Delta_{2}(2)\right|\right) .
\end{aligned}
$$

Note that the above three cases cover all possible situations because if $(d, i)$ is not comparable with $\left(c_{0}, 0\right)$ by the relation $\succeq$, then $d(2) \leq\left|p_{1}\right|=c_{0}(1)$ or $d(1) \geq\left|p_{1}\right|=c_{0}(2)$.

As above, let $\widehat{B}_{1, i}:=\left\{\widehat{d} \mid d \in B_{1, i}\right\}$. Then $\widehat{B}_{1,0}, \ldots, \widehat{B}_{1, \ell_{1}}$ is a sequence of bracket systems for $(W, \Delta)$ that changes the bracket system

$$
\widehat{B}_{1,0}=\widehat{B}_{2, \ell_{2}}=\left\{\left(\left|p_{1}\right|,\left|p_{1} p_{2}\right|, 0,0,0,\left|\Delta_{2}(2)\right|\right)\right\}
$$

into the final bracket system

$$
\widehat{B}_{1, \ell_{1}}=\left(0,\left|p_{1}\right|+\left|p_{2}\right|+\left|p_{3}\right|, 0,0,0,\left|\Delta_{1}(2)\right|+\left|\Delta_{2}(2)\right|\right) .
$$

More specifically, it is straightforward to verify that $\widehat{B}_{1,0}, \ldots, \widehat{B}_{1, \ell_{1}}$ is an operational sequence of bracket systems for $(W, \Delta)$ which corresponds to an analogue $\widehat{\Omega}_{1}$ of the sequence of elementary operations $\Omega_{1}$ for $\left(W_{1}, \Delta_{1}\right)$ so that if a bracket $b \in B_{1, i}$, $i \geq 1$, is obtained from brackets $d_{1}, d_{2} \in B_{1, i-1}$, where one of $d_{1}, d_{2}$ could be missing, 
by an elementary operation $\sigma$ of $\Omega_{1}$, then $\widehat{b} \in \widehat{B}_{1, i}$ is obtained from $\widehat{d}_{1}, \widehat{d}_{2} \in \widehat{B}_{1, i-1}$ by an elementary operation $\widehat{\sigma}$ of $\widehat{\Omega}_{1}$ and $\widehat{\sigma}$ has the same type as $\sigma$.

Thus, with the indicated changes, we can now combine the foregoing sequences of bracket systems for $\left(W_{2}, \Delta_{2}\right)$ and for $\left(W_{1}, \Delta_{1}\right)$ into a single sequence of bracket systems for $(W, \Delta)$ that transforms the empty bracket system into the bracket system $\left\{\left(\left|p_{1}\right|,\left|p_{1} p_{2}\right|, 0,0,0,\left|\Delta_{2}(2)\right|\right)\right\}$ and then continues to turn the latter into the final bracket system. It follows from definitions and bounds (4.11)-(4.13) that the size of thus constructed sequence is bounded by

$$
\left(11\left|W_{1}\right|+11\left|W_{2}\right|+1, \max \left(C\left(\log \left|W_{1}\right|+1\right)+1, C\left(\log \left|W_{2}\right|+1\right)\right)\right)
$$

Therefore, in view of Lemma 4.4 it remains to show that

$$
\max \left(C\left(\log \left|W_{1}\right|+1\right)+1, C\left(\log \left|W_{2}\right|+1\right)\right) \leq C(\log |W|+1) .
$$

In view of inequality (4.10),

$$
\max \left(C\left(\log \left|W_{1}\right|+1\right)+1, C\left(\log \left|W_{2}\right|+1\right)\right) \leq C\left(\log \left(\frac{5}{6}|W|\right)+1\right)+1,
$$

and $C\left(\log \left(\frac{5}{6}|W|\right)+1\right)+1 \leq C(\log |W|+1)$ if $C \geq\left(\log \frac{6}{5}\right)^{-1}$. Thus the first main case, when Lemma 4.2 (a) holds for the pair $(W, \Delta)$, is complete.

Now assume that Lemma 4.2(b) holds true for the pair $(W, \Delta)$ and $\Pi$ is the face in $\Delta$ with $|\partial \Pi| \geq 2, v_{1}, v_{2} \in P_{W}$ are the vertices of $P_{W}$ such that $v_{1}<v_{2}$, $\alpha\left(v_{1}\right), \alpha\left(v_{2}\right) \in \partial \Pi$, and if $P_{W}\left(\right.$ fact, $\left.v_{1}, v_{2}\right)=p_{1} p_{2} p_{3}$ then

$$
\min \left(\left|p_{2}\right|,\left|p_{1}\right|+\left|p_{3}\right|\right) \geq \frac{1}{6}|W| \text {. }
$$

Furthermore, if $(\partial \Pi)^{-1}=e_{1} \ldots e_{|\partial \Pi|}$, where $e_{i} \in \vec{\Delta}(1)$, and the cyclic boundary $\partial \Delta$ of $\Delta$ is $\partial \Delta=e_{1} h_{1} \ldots e_{|\partial \Pi|} h_{|\partial \Pi|}$, where $h_{i}$ is a closed subpath of $\partial \Delta$, then, for every $i, h_{i}$ is a subpath of either $\alpha\left(p_{2}\right)$ or $\alpha\left(p_{3}\right) \alpha\left(p_{1}\right)$ and $\left|h_{i}\right| \leq \frac{5}{6}|W|$.

For $i=2, \ldots,|\partial \Pi|$, denote $W_{i}:=\varphi\left(h_{i}\right)$ and let $\Delta_{i}$ be the disk subdiagram of $\Delta$ with $\left.\partial\right|_{\left(h_{i}\right)_{-}} \Delta_{i}=h_{i}$. We also consider a path $P_{W_{i}}$ with $\varphi\left(P_{W_{i}}\right) \equiv W_{i}$ and let $\alpha_{i}: P_{W_{i}} \rightarrow \Delta_{i}$ denote the map whose definition is analogous to that of $\alpha: P_{W} \rightarrow \Delta$ (note that $\left|W_{i}\right|=0$ is now possible).

By the induction hypothesis on $|W|$ applied to $\left(W_{i}, \Delta_{i}\right)$ (the case $\left|W_{i}\right|=0$ is vacuous), there is a sequence $\Omega_{i}$ of elementary operations for $\left(W_{i}, \Delta_{i}\right)$ that transforms the empty bracket system into the final one and has size bounded by $\left(10\left|W_{i}\right|, C\left(\log \left|W_{i}\right|+1\right)\right)$.

Making a cyclic permutation of indices of $e_{i}, h_{i}$ in $\partial \Delta=e_{1} h_{1} \ldots e_{|\partial \Pi|} h_{|\partial \Pi|}$ if necessary, we may assume that

$$
P_{W}=s_{2} f_{2} q_{2} f_{3} q_{3} \ldots f_{|\partial \Pi|} q_{|\partial \Pi|} f_{1} s_{1},
$$

where $\alpha\left(f_{i}\right)=e_{i}, i=1, \ldots,|\partial \Pi|, \alpha\left(q_{j}\right)=h_{j}, j=2, \ldots,|\partial \Pi|$, and $\alpha\left(s_{1}\right) \alpha\left(s_{2}\right)=h_{1}$, see Fig. 4.2. Note that $\left|s_{1}\right|=0$ or $\left|s_{2}\right|=0$ is possible.

Let $B_{i, 0}, \ldots, B_{i, \ell_{i}}$ denote the corresponding to $\Omega_{i}$ sequence of bracket systems, where $B_{i, 0}$ is empty and $B_{i, \ell_{i}}$ is final. As in the above arguments, we can easily convert every bracket $b \in \cup_{j} B_{i, j}$, where $i>1$, into a bracket $\widehat{b}$ for $(W, \Delta)$ by using the rule

$$
\widehat{b}:=\left(b(1)+\left|s_{2} f_{2} \ldots f_{i}\right|, b(2)+\left|s_{2} f_{2} \ldots f_{i}\right|, b(3), b(4), b(5), b(6)\right) .
$$

Then the sequence $\widehat{B}_{i, 0}, \ldots, \widehat{B}_{i, \ell_{i}}$, where $\widehat{B}_{i, j}:=\left\{\widehat{b} \mid b \in B_{i, j}\right\}$, becomes an operational sequence of bracket systems for $(W, \Delta)$ that transforms the empty bracket 
system into $\widehat{B}_{i, \ell_{i}}=\left\{\widehat{d}_{i}\right\}$, where

$$
\left.\widehat{d}_{i}=\left(\left|s_{2} f_{2} \ldots f_{i}\right|,\left|s_{2} f_{2} \ldots f_{i} q_{i}\right|, 0,0,0,\left|\Delta_{i}(2)\right|\right)\right\}, i>1 .
$$

We also remark that the sequence of bracket systems $\widehat{B}_{i, 0}, \ldots, \widehat{B}_{i, \ell_{i}}$ corresponds to an analogue $\widehat{\Omega}_{i}$ of the sequence of elementary operations $\Omega_{i}$ for $\left(W_{i}, \Delta_{i}\right)$ so that if a bracket $b \in B_{i, j}, j \geq 1$, is obtained from brackets $g_{1}, g_{2} \in B_{i, j-1}$, where one of $g_{1}, g_{2}$ could be missing, by an elementary operation $\sigma$ of $\Omega_{i}$, then $\widehat{b} \in \widehat{B}_{i, j}$ is obtained from $\widehat{g}_{1}, \widehat{g}_{2} \in \widehat{B}_{i, j-1}$ by an elementary operation $\widehat{\sigma}$ of $\widehat{\Omega}_{i}$ and $\widehat{\sigma}$ has the same type as $\sigma$.

Denote $\varphi\left((\partial \Pi)^{-1}\right)=a_{j_{\Pi}}^{\varepsilon n_{\Pi}}$. Using the operational sequence $\widehat{B}_{2,0}, \ldots, \widehat{B}_{2, \ell_{2}}$, we convert the empty bracket system into $\left\{\widehat{d}_{2}\right\}$. Applying a turn operation to $\widehat{d}_{2}$, we change $\widehat{d}_{2}(3)$ from 0 to $j_{\Pi}$ and $d_{2}(4)$ from 0 to $n_{\Pi}$. Note that $n_{\Pi} \leq|W|$ by Lemma 2.2, hence this is correct to do so. Then we apply two extensions of type 1 on the left and on the right to increase $\widehat{d}_{2}(2)$ by 1 and to decrease $\widehat{d}_{2}(1)$ by 1 , see Fig. 4.2. Let

$$
\widetilde{d}_{2}=\left(\left|s_{2}\right|,\left|s_{2} f_{2} q_{2} f_{3}\right|, j_{\Pi}, n_{\Pi}, 2 \varepsilon,\left|\Delta_{2}(2)\right|\right)
$$

denote the bracket of type B2 obtained this way. Now, starting with the bracket system $\left\{\widetilde{d}_{2}\right\}$, we apply those elementary operations that are used to create the sequence $\widehat{B}_{3,0}, \ldots, \widehat{B}_{3, \ell_{3}}$, and obtain the bracket system $\left\{\widetilde{d}_{2}, \widehat{d}_{3}\right\}$. Applying a merger to $\widetilde{d}_{2}, \widehat{d}_{3}$, we get

$$
\widehat{d_{3}^{\prime}}=\left(\left|s_{2}\right|,\left|s_{2} f_{2} q_{2} f_{3} q_{3}\right|, j_{\Pi}, n_{\Pi}, 2 \varepsilon,\left|\Delta_{2}(2)\right|+\left|\Delta_{3}(2)\right|\right) .
$$

Let $\widetilde{d}_{3}$ be obtained from $\widehat{d}_{3}^{\prime}$ by extension of type 1 on the right, so

$$
\widetilde{d}_{3}=\left(\left|s_{2}\right|,\left|s_{2} f_{2} q_{2} f_{3} q_{3} f_{4}\right|, j_{\Pi}, n_{\Pi}, 3 \varepsilon,\left|\Delta_{2}(2)\right|+\left|\Delta_{3}(2)\right|\right) .
$$

Iterating in this manner, we will arrive at a bracket system consisting of the single bracket

$$
\widehat{d}_{|\partial \Pi|}^{\prime}=\left(\left|s_{2}\right|,\left|s_{2} f_{2} \ldots q_{|\partial \Pi|}\right|, j_{\Pi}, n_{\Pi}, \varepsilon(|\partial \Pi|-1), \sum_{i \geq 2}\left|\Delta_{i}(2)\right|\right) .
$$

Applying to $\widehat{d}_{|\partial \Pi|}^{\prime}$ an extension of type 2 on the right along the edge $f_{1}=\alpha\left(e_{1}\right)$, see Fig. 4.2, we obtain the bracket

$$
\widetilde{d}_{|\partial \Pi|}=\left(\left|s_{2}\right|,|W|-\left|s_{1}\right|, 0,0,0,1+\sum_{i \geq 2}\left|\Delta_{i}(2)\right|\right)
$$

of type B1.

For $i=1$, we let $W_{1}:=\varphi\left(s_{2}\right) \varphi\left(s_{1}\right)$ and let $\Delta_{1}$ be the disk subdiagram of $\Delta$ with

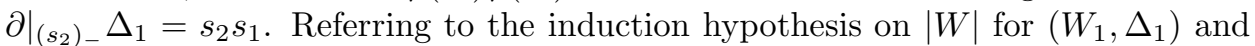
to Lemma 4.5. we conclude that there is a sequence $\Omega_{1}^{\prime}$ of elementary operations that changes the starting bracket

$$
c_{2}:=\left(\left|s_{2}\right|,\left|s_{2}\right|, 0,0,0,0\right)
$$

into the final bracket system and has size bounded by

$$
\left(11\left|W_{1}\right|+1, C\left(\log \left|W_{1}\right|+1\right)+1\right) .
$$

Let $B_{1,0}^{\prime}, \ldots, B_{1, \ell_{1}^{\prime}}^{\prime}$ denote the corresponding to $\Omega_{1}^{\prime}$ sequence of bracket systems, where $B_{1,0}^{\prime}=\left\{c_{2}\right\}$ and $B_{1, \ell_{1}^{\prime}}^{\prime}$ is final. Similarly to the above construction of $\widehat{B}_{1, i}$ from $B_{1, i}$, we will make changes over brackets $b \in \cup_{j} B_{1, j}^{\prime}$ so that every $b$ becomes a 
bracket $\widehat{b}$ for $(W, \Delta)$ and if $\widehat{B}_{1, i}^{\prime}:=\left\{\widehat{b} \mid b \in B_{1, i}^{\prime}\right\}$, then the sequence $\widehat{B}_{1,0}^{\prime}, \ldots, \widehat{B}_{1, \ell_{1}^{\prime}}^{\prime}$ transforms the bracket system $\widehat{B}_{1,0}^{\prime}=\left\{\widehat{c}_{2}\right\}=\left\{\widetilde{d}_{|\partial \Pi|}\right\}$ into the final one for $(W, \Delta)$.

Specifically, define a relation $\succeq^{\prime}$ on the set of all pairs $(b, i)$, where $b \in B_{1, i}^{\prime}$, that is reflexive and transitive closure of the relation $(c, i+1) \succ^{\prime}(b, i)$, where a bracket $c \in B_{1, i+1}$ is obtained from brackets $b, b^{\prime} \in B_{1, i}$ by an elementary operation $\sigma$, where $b^{\prime}$ could be missing. As before, it follows from the definitions that $\succeq^{\prime}$ is a partial order on the set of all such pairs $(b, i)$ and that if $\left(b_{2}, i_{2}\right) \succeq^{\prime}\left(b_{1}, i_{1}\right)$ then $i_{2} \geq i_{1}$ and

$$
b_{2}(1) \leq b_{1}(1) \leq b_{1}(2) \leq b_{2}(2) .
$$

Furthermore, every bracket $b=(b(1), \ldots, b(6))$, where $b \in B_{1, i}^{\prime}$, for $\left(W_{1}, \Delta_{1}\right)$, naturally gives rise to a bracket

$$
\widehat{b}=(\widehat{b}(1), \ldots, \widehat{b}(6))
$$

for $(W, \Delta)$ in the following fashion.

If the pair $(b, i)$ is not comparable with $\left(c_{2}, 0\right)$, where $c_{2}$ is defined in (4.14), by the relation $\succeq^{\prime}$ and $b(2) \leq\left|s_{2}\right|$, then

$$
\widehat{b}:=b \text {. }
$$

If the $(b, i)$ is not comparable with $\left(c_{2}, 0\right)$ by the relation $\succeq^{\prime}$ and $\left|s_{1}\right| \leq b(1)$, then

$$
\widehat{b}:=\left(b(1)+|W|-\left|h_{1}\right|, b(2)+|W|-\left|h_{1}\right|, b(3), b(4), b(5), b(6)\right) .
$$

If $(d, i) \succeq^{\prime}\left(c_{2}, 0\right)$, then

$$
\widehat{b}:=\left(b(1), b(2)+|W|-\left|h_{1}\right|, b(3), b(4), b(5), b(6)+1+\sum_{i \geq 2}\left|\Delta_{i}(2)\right|\right) .
$$

As before, we note that these three cases cover all possible situations because if $(b, i)$ is not comparable with $\left(c_{2}, 0\right)$ by the relation $\succeq^{\prime}$, then either

$$
b(2) \leq|W|-\left|h_{1}\right|=c_{2}(1)
$$

or $b(1) \geq|W|-\left|h_{1}\right|=c_{2}(2)$.

Similarly to the foregoing arguments, we check that $\widehat{B}_{1,0}^{\prime}, \ldots, \widehat{B}_{1, \ell_{1}}^{\prime}$ is an operational sequence of bracket systems for $(W, \Delta)$ which corresponds to an analogue $\widehat{\Omega}_{1}^{\prime}$ of the sequence of elementary operations $\Omega_{1}^{\prime}$ for $\left(W_{1}, \Delta_{1}\right)$ so that if a bracket $b \in B_{1, i}^{\prime}, i \geq 1$, is obtained from brackets $g_{1}, g_{2} \in B_{1, i-1}^{\prime}$, where one of $g_{1}, g_{2}$ could be missing, by an elementary operation $\sigma$ of $\Omega_{1}^{\prime}$, then $\widehat{b} \in \widehat{B}_{1, i}^{\prime}$ is obtained from $\widehat{g}_{1}, \widehat{g}_{2} \in \widehat{B}_{1, i-1}^{\prime}$ by an elementary operation $\widehat{\sigma}$ of $\widehat{\Omega}_{1}^{\prime}$ and $\widehat{\sigma}$ has the same type as $\sigma$.

Summarizing, we conclude that there exists a sequence of elementary operations $\Omega$, containing subsequences $\widehat{\Omega}_{2}, \ldots, \widehat{\Omega}_{|\partial \Pi|}$, that transforms the empty bracket system for $(W, \Delta)$ into the bracket system $B=\left\{\widetilde{d}_{|\partial \Pi|}\right\}$ and then, via subsequence $\widehat{\Omega}_{1}^{\prime}$, continues to transform

$$
B=\left\{\widetilde{d}_{|\partial \Pi|}\right\}=\left\{\widehat{c}_{2}\right\}
$$

into the final bracket system for $(W, \Delta)$. It follows from the induction hypothesis for the pairs $\left(W_{i}, \Delta_{i}\right)$ and definitions that the size of thus constructed sequence $\Omega$ is bounded by

$$
\left(\left(11 \cdot \sum_{1 \leq i \leq|\partial \Pi|}\left|W_{i}\right|\right)+2|\partial \Pi|+2, \max _{1 \leq i \leq|\partial \Pi|}\left\{C\left(\log \left|W_{i}\right|+1\right)+1\right\}\right) .
$$


In view of Lemma 4.4, it suffices to show that

$$
\max _{1 \leq i \leq|\partial \Pi|}\left\{C\left(\log \left|W_{i}\right|+1\right)+1\right\} \leq C(\log |W|+1) .
$$

Since $\left|W_{i}\right| \leq \frac{5}{6}|W|$, the latter inequality holds true, as in the above case, for $C=$ $\left(\log \frac{5}{6}\right)^{-1}$.

Let $W$ be an arbitrary nonempty word over the alphabet $\mathcal{A}^{ \pm 1}$, not necessarily representing the trivial element of the group given by presentation (1.2) (and $W$ is not necessarily reduced). As before, let $P_{W}$ be a labeled simple path with $\varphi\left(P_{W}\right) \equiv$ $W$ and let vertices of $P_{W}$ be identified along $P_{W}$ with integers $0, \ldots,|W|$ so that $\left(P_{W}\right)_{-}=0, \ldots,\left(P_{W}\right)_{+}=|W|$.

A 6-tuple

$$
b=(b(1), b(2), b(3), b(4), b(5), b(6))
$$

of integers $b(1), b(2), b(3), b(4), b(5), b(6)$ is called a pseudobracket for the word $W$ if $b(1), b(2)$ satisfy the inequalities $0 \leq b(1) \leq b(2) \leq|W|$ and one of the following two properties (PB1), (PB2) holds for $b$.

$(\mathrm{PB} 1) \quad b(3)=b(4)=b(5)=0$ and $0 \leq b(6) \leq|W|$.

(PB2) $b(3) \in\{1, \ldots, m\}, b(4) \in E_{b(3)}$, where the set $E_{b(3)}$ is defined in (1.2), $0<b(4) \leq|W|,-b(4)<b(5)<b(4)$, and $0 \leq b(6) \leq|W|$.

We say that a pseudobracket $b$ has type $P B 1$ if the property (PB1) holds for $b$. We also say that a pseudobracket $b$ has type PB2 if the property (PB2) holds for $b$. Clearly, these two types are mutually exclusive.

Let $p$ denote the subpath of $P_{W}$ such that $p_{-}=b(1)$ and $p_{+}=b(2)$, perhaps, $p_{-}=p_{+}$and $|p|=0$. The subpath $p$ of $P_{W}$ is denoted $a(b)$ and is called the arc of the pseudobracket $b$.

For example, $b_{v}=(v, v, 0,0,0,0)$ is a pseudobracket of type $\mathrm{PB} 1$ for every vertex $v \in P_{W}$ and such $b_{v}$ is called a starting pseudobracket. Note that $a(b)=\{v\}$. A final pseudobracket for $W$ is $c=(0,|W|, 0,0,0, k)$, where $k \geq 0$ is an integer. Note that $a(c)=P_{W}$.

Observe that if $b$ is a bracket for the pair $(W, \Delta)$ of type $\mathrm{B} 1$ or $\mathrm{B} 2$, then $b$ is also a pseudobracket of type PB1 or PB2, resp., for the word $W$.

Let $B$ be a finite set of pseudobrackets for $W$, perhaps, $B$ is empty. We say that $B$ is a pseudobracket system for $W$ if, for every pair $b, c \in B$ of distinct pseudobrackets, either $b(2) \leq c(1)$ or $c(2) \leq b(1)$. It follows from the definitions that every bracket system for $(W, \Delta)$ is also a pseudobracket system for the word $W$. $B$ is called a final pseudobracket system for $W$ if $B$ consists of a single final pseudobracket for $W$.

Now we describe four kinds of elementary operations over pseudobrackets and over pseudobracket systems: additions, extensions, turns, and mergers, which are analogous to those definitions for brackets and bracket systems, except there are no any diagrams and no faces involved.

Let $B$ be a pseudobracket system for a nonempty word $W$ over $\mathcal{A}^{ \pm 1}$.

Additions.

Suppose $b$ is a starting pseudobracket, $b \notin B$, and $B \cup\{b\}$ is a pseudobracket system. Then we may add $b$ to $B$ thus making an addition operation over $B$.

Extensions. 
Suppose $b \in B, b=(b(1), b(2), b(3), b(4), b(5), b(6))$, and $e_{1} a(b) e_{2}$ is a subpath of $P_{W}$, where $a(b)$ is the arc of $b$ and $e_{1}, e_{2}$ are edges one of which could be missing.

Assume that $b$ is of type PB2. Suppose $\varphi\left(e_{1}\right)=a_{b(3)}^{\varepsilon_{1}}$, where $\varepsilon_{1}= \pm 1$.

If $|b(5)| \leq b(4)-2$ and $\varepsilon_{1} b(5) \geq 0$, then we consider a pseudobracket $b^{\prime}$ of type PB2 such that

$$
\begin{aligned}
& b^{\prime}(1)=b(1)-1, b^{\prime}(2)=b(2), b^{\prime}(3)=b(3), \\
& b^{\prime}(4)=b(4), b^{\prime}(5)=b(5)+\varepsilon_{1}, b^{\prime}(6)=b(6) .
\end{aligned}
$$

Note that $a\left(b^{\prime}\right)=e_{1} a(b)$. We say that $b^{\prime}$ is obtained from $b$ by an extension of type 1 (on the left). If $(B \backslash\{b\}) \cup\left\{b^{\prime}\right\}$ is a pseudobracket system, then replacement of $b \in B$ with $b^{\prime}$ in $B$ is called an extension operation over $B$ of type 1 .

On the other hand, if $|b(5)|=b(4)-1$ and $\varepsilon_{1} b(5) \geq 0$, then we consider a pseudobracket $b^{\prime}$ of type PB1 such that

$$
b^{\prime}(1)=b(1)-1, b^{\prime}(2)=b(2), b^{\prime}(3)=b^{\prime}(4)=b^{\prime}(5)=0, b^{\prime}(6)=b(6)+1 .
$$

In this case, we say that $b^{\prime}$ is obtained from $b$ by an extension of type 2 (on the left). Note that $a\left(b^{\prime}\right)=e_{1} a(b)$ and $b^{\prime}$ has type PB1. If $(B \backslash\{b\}) \cup\left\{b^{\prime}\right\}$ is a pseudobracket system, then replacement of $b \in B$ with $b^{\prime}$ in $B$ is called an extension operation over $B$ of type 2 .

Analogously, assume that $b$ has type PB2 and $\varphi\left(e_{2}\right)=a_{b(3)}^{\varepsilon_{2}}$, where $\varepsilon_{2}= \pm 1$.

If $|b(5)| \leq b(4)-2$ and $\varepsilon_{2} b(5) \geq 0$, then we consider a pseudobracket $b^{\prime}$ such that

$$
\begin{aligned}
& b^{\prime}(1)=b(1), b^{\prime}(2)=b(2)+1, b^{\prime}(3)=b(3), \\
& b^{\prime}(4)=b(4), b^{\prime}(5)=b(5)+\varepsilon_{2}, b^{\prime}(6)=b(6) .
\end{aligned}
$$

Note that $a\left(b^{\prime}\right)=a(b) e_{2}$ and $b^{\prime}$ has type PB2. We say that $b^{\prime}$ is obtained from $b$ by an extension of type 1 (on the right). If $(B \backslash\{b\}) \cup\left\{b^{\prime}\right\}$ is a pseudobracket system, then replacement of $b \in B$ with $b^{\prime}$ in $B$ is called an extension operation over $B$ of type 1 .

On the other hand, if $|b(5)|=b(4)-1$ and $\varepsilon_{2} b(5) \geq 0$, then we consider a pseudobracket $b^{\prime}$ such that

$$
b^{\prime}(1)=b(1), b^{\prime}(2)=b(2)+1, b^{\prime}(3)=b^{\prime}(4)=b^{\prime}(5)=0, b^{\prime}(6)=b(6)+1 .
$$

Note that $a\left(b^{\prime}\right)=a(b) e_{2}$ and $b^{\prime}$ has type PB1. We say that $b^{\prime}$ is obtained from $b$ by an extension of type 2 (on the right). If $(B \backslash\{b\}) \cup\left\{b^{\prime}\right\}$ is a pseudobracket system, then replacement of $b \in B$ with $b^{\prime}$ in $B$ is called an extension operation over $B$ of type 2 .

Assume that $b \in B$ is a pseudobracket of type PB1, $e_{1} a(b) e_{2}$ is a subpath of $P_{W}$, where $a(b)$ is the arc of $b$ and $e_{1}, e_{2}$ are edges with $\varphi\left(e_{1}\right)=\varphi\left(e_{2}\right)^{-1}$. Consider a pseudobracket $b^{\prime}$ of type PB1 such that

$$
b^{\prime}(1)=b(1)-1, b^{\prime}(2)=b(2)+1, b^{\prime}(3)=b^{\prime}(4)=b^{\prime}(5)=0, b^{\prime}(6)=b(6) .
$$

Note that $a\left(b^{\prime}\right)=e_{1} a(b) e_{2}$. We say that $b^{\prime}$ is obtained from $b$ by an extension of type 3. If $(B \backslash\{b\}) \cup\left\{b^{\prime}\right\}$ is a pseudobracket system, then replacement of $b \in B$ with $b^{\prime}$ in $B$ is called an extension operation over $B$ of type 3 .

Turns.

Let $b \in B$ be a pseudobracket of type PB1. Then, by the definition, $b(3)=b(4)=$ $b(5)=0$. Pick $j \in\{1, \ldots, m\}$ such that $E_{j} \neq\{0\}$. Consider a pseudobracket $b^{\prime}$ with $b^{\prime}(i)=b(i)$ for $i=1,2,4,5,6$, and $b^{\prime}(3)=j$. Note that $b^{\prime}$ is of type PB2 and 
$a\left(b^{\prime}\right)=a(b)$. We say that $b^{\prime}$ is obtained from $b$ by a turn operation. Replacement of $b \in B$ with $b^{\prime}$ in $B$ is also called a turn operation over $B$. Note that $(B \backslash\{b\}) \cup\left\{b^{\prime}\right\}$ is a pseudobracket system because so is $B$.

Mergers.

Now suppose that $b, c \in B$ are distinct pseudobrackets such that $b(2)=c(1)$ and one of $b(3), c(3)$ is 0 . Then one of $b, c$ is of type PB1 and the other has type $\mathrm{PB} 1$ or PB2. Consider a pseudobracket $b^{\prime}$ such that $b^{\prime}(1)=b(1), b^{\prime}(2)=c(2)$, and $b^{\prime}(i)=b(i)+c(i)$ for $i=3,4,5,6$. Note that $a\left(b^{\prime}\right)=a(b) a(c)$ and $b^{\prime}$ is of type PB1 if both $b, c$ have type PB1 or $b^{\prime}$ is of type PB2 if one of $b, c$ has type PB2. We say that $b^{\prime}$ is obtained from $b, c$ by a merger operation. Taking both $b, c$ out of $B$ and putting $b^{\prime}$ in $B$ is a merger operation over $B$.

As before, we will say that additions, extensions, turns and mergers, as defined above, are elementary operations over pseudobrackets and pseudobracket systems for $W$.

Assume that one pseudobracket system $B_{\ell}$ is obtained from another pseudobracket system $B_{0}$ by a finite sequence $\Omega$ of elementary operations and $B_{0}, B_{1}, \ldots, B_{\ell}$ is the corresponding to $\Omega$ sequence of pseudobracket systems. Such a sequence $B_{0}, B_{1}, \ldots, B_{\ell}$ of pseudobracket systems is called operational. We say that a sequence $B_{0}, B_{1}, \ldots, B_{\ell}$ of pseudobracket systems has size bounded by $\left(k_{1}, k_{2}\right)$ if $\ell \leq k_{1}$ and, for every $i$, the number of pseudobrackets in $B_{i}$ is at most $k_{2}$. Whenever it is not ambiguous, we also say that $\Omega$ has size bounded by $\left(k_{1}, k_{2}\right)$ if so does the corresponding to $\Omega$ sequence $B_{0}, B_{1}, \ldots, B_{\ell}$ of pseudobracket systems.

The significance of pseudobracket systems and elementary operations over pseudobracket systems introduced above is revealed in the following lemma.

Lemma 4.7. Suppose that the empty pseudobracket system $B_{0}$ for $W$ can be transformed by a finite sequence $\Omega$ of elementary operations into a final pseudobracket system $\left\{b_{F}\right\}$. Then $W \stackrel{\mathcal{G}_{2}}{=} 1$, i.e., $W$ represents the trivial element of the group defined by presentation (1.2), and there is a disk diagram $\Delta$ over (1.2) such that $\Delta$ has property $(A), \varphi\left(\left.\partial\right|_{0} \Delta\right) \equiv W$ and $|\Delta(2)|=b_{F}(6)$.

Proof. Let $B_{0}, B_{1}, \ldots, B_{\ell}$ be the corresponding to $\Omega$ sequence of pseudobracket systems, where $B_{0}$ is empty and $B_{\ell}$ is final. Consider the following Claims (C1)(C2) for a pseudobracket $b \in B_{i}, i=1, \ldots, \ell$, in which $a(b)$ denotes the arc of $b$.

(C1) If a pseudobracket $b \in B_{i}$ has type PB1 then $\varphi(a(b)) \stackrel{\mathcal{G}_{2}}{=} 1$ in the group $\mathcal{G}_{2}$ given by presentation (1.2) and there is a disk diagram $\Delta_{b}$ over (1.2) such that $\Delta_{b}$ has property $(\mathrm{A}), \varphi\left(\partial \Delta_{b}\right) \equiv \varphi(a(b))$ and $\left|\Delta_{b}(2)\right|=b(6)$.

(C2) If a pseudobracket $b \in B_{i}$ has type PB2, then $\varphi(a(b)) \stackrel{\mathcal{G}_{2}}{=} a_{b(3)}^{b(5)}$ and there is a disk diagram $\Delta_{b}$ over (1.2) such that $\Delta_{b}$ has property (A), $\partial \Delta_{b}=p q^{-1}$, where $p, q^{-1}$ are subpaths of $\partial \Delta_{b}, \varphi(p) \equiv \varphi(a(b)), \varphi(q) \equiv a_{b(3)}^{b(5)},\left|\Delta_{b}(2)\right|=$ $b(6)$, and if $e$ is an edge of $q^{-1}$ then $e^{-1} \in p$.

By induction on $i \geq 1$, we will prove that Claims $(\mathrm{C} 1)-(\mathrm{C} 2)$ hold true for every pseudobracket $b^{\prime} \in B_{i}$. Note that if $b^{\prime}$ is a starting pseudobracket, then $b^{\prime}$ is of type $\mathrm{PB} 1$ and Claim $(\mathrm{C} 1)$ is obviously true for $b^{\prime}$ (Claim $(\mathrm{C} 2)$ is vacuously true for $b^{\prime}$ ). Since $B_{1}$ consists of a starting pseudobracket, the base of induction is established. 
To make the induction step from $i-1$ to $i, i \geq 2$, we consider the cases corresponding to the type of the elementary operation that is used to get $B_{i}$ from $B_{i-1}$.

Suppose that $B_{i}$ is obtained from $B_{i-1}$ by an elementary operation $\sigma$ and $b^{\prime} \in B_{i}$ is the pseudobracket obtained from $b, c \in B_{i-1}$ by application of $\sigma$, here one of $b, c$ or both, depending on type of $\sigma$, could be missing. By the induction hypothesis, Claims (C1)-(C2) hold for every pseudobracket of $B_{i}$ different from $b^{\prime}$ and it suffices to show that the suitable Claim (C1)-(C2) holds for $b^{\prime}$.

If $B_{i}$ is obtained from $B_{i-1}$ by an addition, then it suffices to refer to the above remark that Claims $(\mathrm{C} 1)-(\mathrm{C} 2)$ hold for a starting pseudobracket.

Suppose that $B_{i}$ is obtained from $B_{i-1}$ by an extension of type 1 and let $b^{\prime} \in B_{i}$ be the pseudobracket created from $b \in B_{i-1}$ by an extension of type 1 on the left (the "right" subcase is symmetric).

Note that both $b$ and $b^{\prime}$ have type PB2. By the induction hypothesis, which is Claim (C2) for $b$, there exists a disk diagram $\Delta_{b}$ such that $\Delta_{b}$ has property (A),

$$
\partial \Delta_{b}=p q^{-1}, \quad \varphi(p) \equiv \varphi(a(b)), \quad \varphi(q) \equiv a_{b(3)}^{b(5)}, \quad\left|\Delta_{b}(2)\right|=b(6),
$$

and if $e$ is an edge of $q^{-1}$ then $e^{-1} \in p$. Here and below we use the notation of the definition of an extension of type 1 .

Let $e_{1}$ denote the edge of $P_{W}$ such that $a\left(b^{\prime}\right)=e_{1} a(b)$ and $\varphi\left(e_{1}\right)=a_{b(3)}^{\varepsilon_{1}}, \varepsilon_{1}= \pm 1$. Consider a "loose" edge $f$ with $\varphi(f)=a_{b(3)}^{\varepsilon_{1}}$. We attach the vertex $f_{+}$to the vertex $p_{-}=q_{-}$of $\Delta_{b}$ to get a new disk diagram $\Delta_{b}^{\prime}$ such that $\partial \Delta_{b}^{\prime}=f p q^{-1} f^{-1}$, see Fig. 4.5. Note that property (A) holds for $\Delta_{b}^{\prime}$,

$$
\varphi(f p) \equiv a_{b(3)}^{\varepsilon_{1}} \varphi(a(b)) \equiv \varphi\left(e_{1} a(b)\right) \equiv \varphi\left(a\left(b^{\prime}\right)\right), \quad \varphi\left(q^{-1} f^{-1}\right) \equiv a_{b(3)}^{b(5)+\varepsilon_{1}} \equiv a_{b(3)}^{b^{\prime}(5)},
$$

$\left|\Delta_{b}^{\prime}(2)\right|=\left|\Delta_{b}(2)\right|=b(6)=b^{\prime}(6)$, and if $e$ is an edge of $q^{-1}$ then $e^{-1} \in p$. Thus, Claim (C2) holds for the pseudobracket $b^{\prime}$ with $\Delta_{b^{\prime}}:=\Delta_{b}^{\prime}$.

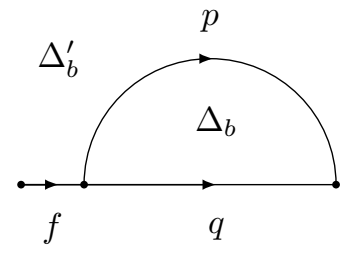

Fig. 4.5

Assume that $B_{i}$ is obtained from $B_{i-1}$ by an extension of type 2 and let $b^{\prime} \in B_{i}$ be the pseudobracket obtained from $b \in B_{i-1}$ by an extension of type 2 on the left (the "right" subcase is symmetric).

Note that $b$ has type PB2, while $b^{\prime}$ has type PB1. By the induction hypothesis, which is Claim (C2) for $b$, there exists a disk diagram $\Delta_{b}$ such that $\Delta_{b}$ has property (A),

$$
\partial \Delta_{b}=p q^{-1}, \quad \varphi(p) \equiv \varphi(a(b)), \quad \varphi(q) \equiv a_{b(3)}^{b(5)}, \quad\left|\Delta_{b}(2)\right|=b(6),
$$

and if $e$ is an edge of $q^{-1}$ then $e^{-1} \in p$. Here and below we use the notation of the definition of an extension of type 2 .

Let $e_{1}$ denote the edge of $P_{W}$ such that $a\left(b^{\prime}\right)=e_{1} a(b)$ and $\varphi\left(e_{1}\right)=a_{b(3)}^{\varepsilon_{1}}, \varepsilon_{1}= \pm 1$. Consider a "loose" edge $f$ with $\varphi(f)=a_{b(3)}^{\varepsilon_{1}}$. We attach the vertex $f_{+}$to $p_{-}=q_{-}$ 
and the vertex $f_{-}$to $p_{+}=q_{+}$of $\Delta_{b}$. Since $\varepsilon_{1} b(5) \geq 0$ and $|b(5)|=b(4)-1$, it follows that $\varepsilon_{1}+b(5)=\varepsilon_{1}|b(4)|$. Therefore, $\varphi(q f) \equiv a_{b(3)}^{\varepsilon_{1} b(4)}$ and we can attach a new face $\Pi$ with $\varphi(\partial \Pi) \equiv a_{b(3)}^{-\varepsilon_{1} b(4)}$ so that the boundary path $\partial \Pi$ is identified with the path $q^{-1} f^{-1}$, see Fig. 4.6. This way we obtain a new disk diagram $\Delta_{b}^{\prime}$ such that $\varphi\left(\left.\partial\right|_{f_{-}} \Delta_{b}^{\prime}\right) \equiv \varphi(f) \varphi(p)$ and $\left|\Delta_{b}^{\prime}(2)\right|=\left|\Delta_{b}(2)\right|+1$, see Fig. 4.6. It follows from construction of $\Delta_{b}^{\prime}$ that property (A) holds for $\Delta_{b}^{\prime}$

$$
\varphi(f p) \equiv a_{b(3)}^{\varepsilon_{1}} \varphi(a(b)) \equiv \varphi\left(e_{1} a(b)\right) \equiv \varphi\left(a\left(b^{\prime}\right)\right) \stackrel{\mathcal{G}_{2}}{=} 1, \quad \varphi\left(q^{-1} f^{-1}\right) \equiv a_{b(3)}^{b(5)+\varepsilon_{1}} \equiv a_{b^{\prime}(3)}^{b^{\prime}(5)},
$$

and $\left|\Delta_{b}^{\prime}(2)\right|=\left|\Delta_{b}(2)\right|+1=b(6)+1=b^{\prime}(6)$. Therefore, Claim (C1) holds for the pseudobracket $b^{\prime}$ if we set $\Delta_{b^{\prime}}:=\Delta_{b}^{\prime}$.

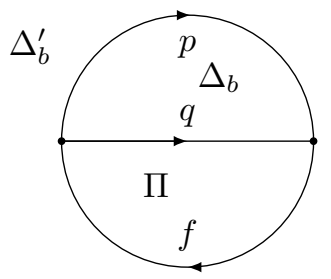

Fig. 4.6

Suppose that an extension of type 3 was applied to get $B_{i}$ from $B_{i-1}$ and $b^{\prime} \in B_{i}$ is the pseudobracket created from $b \in B_{i-1}$ by the operation.

Note that both $b$ and $b^{\prime}$ have type PB1. By the induction hypothesis, which is Claim (C1) for $b$, there exists a disk diagram $\Delta_{b}$ such that $\Delta_{b}$ has property (A) and

$$
\varphi\left(\partial \Delta_{b}\right) \equiv \varphi(a(b)) a_{b(3)}^{-b(5)}, \quad\left|\Delta_{b}(2)\right|=b(6)
$$

Here and below we use the notation of the definition of an extension of type 3 .

Denote $\partial \Delta_{b}=p$, where $\varphi(p) \equiv \varphi(a(b))$ and let $e_{1}, e_{2}$ be the edges of $P_{W}$ such that $a\left(b^{\prime}\right)=e_{1} a(b) e_{2}$ and $\varphi\left(e_{1}\right)=\varphi\left(e_{2}\right)^{-1}$. Consider a "loose" edge $f$ with $\varphi(f)=$ $\varphi\left(e_{1}\right)$. We attach the vertex $f_{+}$to the vertex $p_{-}$of $\Delta_{b}$ to get a new disk diagram $\Delta_{b}^{\prime}$ such that $\partial \Delta_{b}^{\prime}=f p f^{-1}$, see Fig. 4.7. Since $\Delta_{b}$ has property (A),

$$
\varphi\left(\partial \Delta_{b}^{\prime}\right) \equiv \varphi\left(f p f^{-1}\right) \equiv \varphi\left(e_{1}\right) \varphi(a(b)) \varphi\left(e_{1}\right)^{-1} \equiv \varphi\left(a\left(b^{\prime}\right)\right)
$$

and $\left|\Delta_{b}^{\prime}(2)\right|=\left|\Delta_{b}(2)\right|=b(6)=b^{\prime}(6)$, it follows that Claim (C1) holds for the pseudobracket $b^{\prime}$ with $\Delta_{b^{\prime}}:=\Delta_{b}^{\prime}$.

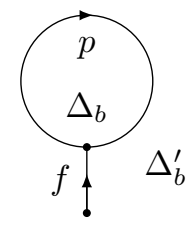

Fig. 4.7

Suppose that $B_{i}$ is obtained from $B_{i-1}$ by a turn operation and let $b^{\prime} \in B_{i}$ be the pseudobracket created from $b \in B_{i-1}$ by the turn operation. By the definition of a turn, $b$ has type PB1 and $b^{\prime}$ has type PB2. By the induction hypothesis, which is Claim (C1) for $b$, there exists a disk diagram $\Delta_{b}$ such that $\Delta_{b}$ has property (A)

$$
\varphi\left(\partial \Delta_{b}\right) \equiv \varphi(a(b)), \quad\left|\Delta_{b}(2)\right|=b(6) .
$$


Here and below we use the notation of the definition of a turn operation.

Denote $\Delta_{b}^{\prime}:=\Delta_{b}$ and let $\partial \Delta_{b}^{\prime}:=p q^{-1}$, where $p, q^{-1}$ are subpaths of $\partial \Delta_{b}^{\prime}$ such that $\varphi(p) \equiv \varphi(a(b))$ and $|q|=0$. Clearly, $\Delta_{b}^{\prime}$ has property (A). Since $a(b)=a\left(b^{\prime}\right)$, $b^{\prime}(5)=b(5)=0$, and

$$
\left|\Delta_{b}^{\prime}(2)\right|=\left|\Delta_{b}(2)\right|=b(6)=b^{\prime}(6),
$$

it follows that $\varphi(p) \equiv \varphi\left(a\left(b^{\prime}\right)\right), \varphi(q) \equiv a_{b^{\prime}(3)}^{b^{\prime}(5)}$. Hence, Claim $(\mathrm{C} 2)$ holds for the pseudobracket $b^{\prime}$ with $\Delta_{b^{\prime}}:=\Delta_{b}^{\prime}$.

Finally, assume that $B_{i}$ results from $B_{i-1}$ by a merger operation and let $b^{\prime} \in B_{i}$ be the pseudobracket created from pseudobrackets $b, c \in B_{i-1}$, where $b(2)=c(1)$, by the operation. By the definition of a merger operation, one of the pseudobrackets $b, c$ must have type PB1. By the induction hypothesis, there are disk diagrams $\Delta_{b}, \Delta_{c}$ for $b, c$, resp., as stated in Claims (C1)-(C2). Denote $\partial \Delta_{b}=p_{b} q_{b}^{-1}, \partial \Delta_{c}=$ $p_{c} q_{c}^{-1}$, where $\varphi\left(p_{b}\right) \equiv \varphi(a(b)), \varphi\left(p_{c}\right) \equiv \varphi(a(c))$, and, for $x \in\{b, c\},\left|q_{x}\right|=0$ if $x$ has type PB1 or $\varphi\left(q_{x}\right) \equiv a_{x(3)}^{x(5)}$ if $x$ has type PB2. Note that $\left|\Delta_{x}(2)\right|=x(6)$.

Consider a disk diagram $\Delta^{\prime}$ obtained from $\Delta_{b}, \Delta_{c}$ by identification of the vertices $\left(p_{b}\right)_{+}$and $\left(p_{c}\right)_{-}$, see Fig. 4.8. Then

$$
\left|\Delta^{\prime}(2)\right|=\left|\Delta_{b}(2)\right|+\left|\Delta_{c}(2)\right|, \quad \partial \Delta^{\prime}=p_{b} p_{c} q_{c}^{-1} q_{b}^{-1} .
$$

Note that

$$
\varphi\left(p_{b} p_{c}\right) \equiv \varphi(a(b)) \varphi(a(c)) \equiv \varphi\left(a\left(b^{\prime}\right)\right), \quad\left|\Delta^{\prime}(2)\right|=b(6)+c(6)
$$

and $\left|q_{c}^{-1} q_{b}^{-1}\right|=0$ if both $b, c$ have type PB1, or $\varphi\left(q_{b} q_{c}\right) \equiv a_{b(3)}^{b(5)}$ if $b$ has type PB2 and $c$ has type PB1, or $\varphi\left(q_{b} q_{c}\right) \equiv a_{c(3)}^{c(5)}$ if $b$ has type PB1 and $c$ has type PB2. Therefore, it follows that Claim (C1) holds true for $b^{\prime}$ with $\Delta_{b^{\prime}}=\Delta^{\prime}$ if both $b, c$ have type PB1 or Claim (C2) holds for $b^{\prime}$ with $\Delta_{b^{\prime}}=\Delta^{\prime}$ if one of $b, c$ has type PB2.

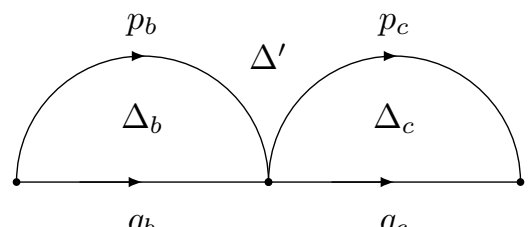

Fig. 4.8

All possible cases are discussed and the induction step is complete. Claims (C1) $-(\mathrm{C} 2)$ are proven.

We can now finish the proof of Lemma 4.7. By Claim (C1) applied to the pseudobracket $b_{F}=\left(0,|W|, 0,0,0, b_{F}(6)\right)$ of the final pseudobracket system $B_{\ell}=$ $\left\{b_{F}\right\}$, there is a disk diagram $\Delta_{b_{F}}$ over (1.2) such that $\Delta_{b_{F}}$ has property (A), $\varphi\left(\left.\partial\right|_{0} \Delta_{b_{F}}\right) \equiv W$ and $\left|\Delta_{b_{F}}(2)\right|=b_{F}(6)$. Thus, $\Delta_{b_{F}}$ is a desired disk diagram and Lemma 4.7 is proven.

Lemma 4.8. Suppose $W$ is a nonempty word over $\mathcal{A}^{ \pm 1}$ and $n \geq 0$ is an integer. Then $W$ is a product of at most $n$ conjugates of words $R^{ \pm 1}$, where $R=1$ is a relation of presentation (1.2), if and only if there is a sequence $\Omega$ of elementary operations such that $\Omega$ transforms the empty pseudobracket system for $W$ into a 
final pseudobracket system $\left\{b_{F}\right\}$, where $b_{F}(6) \leq n$, and $\Omega$ has size bounded by $(11|W|, C(\log |W|+1))$, where $C=\left(\log \frac{6}{5}\right)^{-1}$.

Proof. Assume that $W$ is a product of at most $n$ conjugates of words $R^{ \pm 1}$, where $R=1$ is a relation of presentation (1.2). By Lemmas 2.1 2.2(b), there is a disk diagram $\Delta$ over (1.2) such that $\Delta$ has property (A), $\varphi\left(\left.\partial\right|_{0} \Delta\right) \equiv W$ and $|\Delta(2)| \leq \min (n,|W|)$. Then Lemma 4.6 applies and yields a sequence $\Omega$ of elementary operations over bracket systems for $(W, \Delta)$ that converts the empty bracket system for $(W, \Delta)$ into the final one and has size bounded by $(11|W|, C(\log |W|+1))$. It follows from arguments of Lemma 4.6 and Lemma 2.2(a) that if $b$ is a bracket of one of the intermediate bracket systems, associated with $\Omega$, then $b(4) \leq|W|$. Observe that every bracket $b \in B$ and every intermediate bracket system $B$ for $(W, \Delta)$, associated with $\Omega$, could be considered as a pseudobracket and a pseudobracket system for $W$, resp., we automatically have a desired sequence of pseudobracket systems.

Conversely, the existence of a sequence $\Omega$ of elementary operations over pseudobracket systems, as specified in Lemma 4.8, implies, by Lemma 4.7 that there is a disk diagram $\Delta$ over (1.2) such that $\Delta$ has property (A), $\varphi\left(\left.\partial\right|_{0} \Delta\right) \equiv W$ and $|\Delta(2)|=b_{F}(6) \leq n$. The existence of such a disk diagram $\Delta$ means that $W$ is a product of $|\Delta(2)|=b_{F}(6) \leq n$ conjugates of words $R^{ \pm 1}$, where $R=1$ is a relation of presentation (1.2).

\section{Proofs of Theorem 1.2 and Corollary 1.3}

Theorem 1.2. Let the group $\mathcal{G}_{2}$ be defined by a presentation of the form

$$
\mathcal{G}_{2}:=\left\langle a_{1}, \ldots, a_{m} \| a_{i}^{k_{i}}=1, k_{i} \in E_{i}, i=1, \ldots, m\right\rangle,
$$

where for every $i$, one of the following holds: $E_{i}=\{0\}$ or, for some integer $n_{i}>0$, $E_{i}=\left\{n_{i}\right\}$ or $E_{i}=n_{i} \mathbb{N}=\left\{n_{i}, 2 n_{i}, 3 n_{i}, \ldots\right\}$. Then both the bounded and precise word problems for (1.2) are in $L^{3}$ and in $P$. Specifically, the problems can be solved in deterministic space $O\left((\log |W|)^{3}\right)$ or in deterministic time $O\left(|W|^{4} \log |W|\right)$.

Proof. We start with $\mathrm{L}^{3}$ part of Theorem 1.2, First we discuss a nondeterministic algorithm which solves the bounded word problem for presentation (1.2) and which is based on Lemma 4.8 .

Given an input $\left(W, 1^{n}\right)$, where $W$ is a nonempty word (not necessarily reduced) over the alphabet $\mathcal{A}^{ \pm 1}$ and $n \geq 0$ is an integer, written in unary notation as $1^{n}$, we begin with the empty pseudobracket system and nondeterministically apply a sequence of elementary operations of size bounded by $(11|W|, C(\log |W|+1))$, where $C=\left(\log \frac{6}{5}\right)^{-1}$. If such a sequence of elementary operations results in a final pseudobracket system $\left\{\left(0,|W|, 0,0,0, n^{\prime}\right)\right\}$, where $n^{\prime} \leq n$, then our algorithm accepts and, in view of Lemma 4.8, we may conclude that $W$ is a product of $n^{\prime} \leq n$ conjugates of words $R^{ \pm 1}$, where $R=1$ is a relation of (1.2). It follows from the definitions and Lemma 4.8 that the number of elementary operations needed for this algorithm to accept is at most $11|W|$. Hence, it follows from the definition of elementary operations over pseudobracket systems for $W$ that the time needed to run this nondeterministic algorithm is $O(|W|)$. To estimate the space requirements of this algorithm, we note that if $b$ is a pseudobracket for $W$, then $b(1), b(2)$ are integers in the range from 0 to $|W|$, hence, when written in binary notation, will take at most $C^{\prime}(\log |W|+1)$ space, where $C^{\prime}$ is a constant. Since $b(3), b(4), b(5), b(6)$ 
are also integers that satisfy inequalities

$$
0 \leq b(3) \leq m, 0 \leq b(4) \leq|W|,|b(5)| \leq b(4), 0 \leq b(6) \leq|W|,
$$

and $m$ is a constant, it follows that the total space required to run this algorithm is at most

$$
5 C^{\prime}(\log |W|+1+\log m) C(\log |W|+1)=O\left((\log |W|)^{2}\right) .
$$

Note that this bound is independent of $n$ because it follows from Lemma 2.2(b) that if $W$ is a product of $n^{\prime}$ conjugates of words $R^{ \pm 1}$, where $R=1$ is a relation of (1.2), then it is possible to assume that $n^{\prime} \leq|W|$.

Furthermore, according to Savitch's theorem 38, see also 3], 32, the existence of a nondeterministic algorithm that recognizes a language in space $S$ and time $T$ implies the existence of a deterministic algorithm that recognizes the language in space $O(S \log T)$. Therefore, by Savitch's theorem [38, there is a deterministic algorithm that solves the bounded word problem for presentation (1.2) in space $O\left((\log |W|)^{3}\right)$.

To solve the precise word problem for presentation (1.2), suppose that we are given a pair $\left(W, 1^{n}\right)$ and wish to find out if $W$ is a product of $n$ conjugates of words $R^{ \pm 1}$, where $R=1$ is a relation of (1.2), and $n$ is minimal with this property. By Lemma 2.2. we may assume that $n \leq|W|$. Using the foregoing deterministic algorithm, we consecutively check whether the bounded word problem is solvable for the two pairs $\left(W, 1^{n-1}\right)$ and $\left(W, 1^{n}\right)$. It is clear that the precise word problem for the pair $\left(W, 1^{n}\right)$ has a positive solution if and only if the bounded word problem has a negative solution for the pair $\left(W, 1^{n-1}\right)$ and has a positive solution for the pair $\left(W, 1^{n}\right)$ and that these two facts can be verified in deterministic space $O\left((\log |W|)^{3}\right)$.

Now we describe an algorithm that solves the precise word problem for presentation (1.2) in polynomial time. Our arguments are analogous to folklore arguments [35], 36] that solve the precise word problem for presentation $\langle a, b \| a=1, b=1\rangle$ in polynomial time and that are based on the method of dynamic programming.

For a word $U$ over $\mathcal{A}^{ \pm 1}$ consider the following property.

(E) $U$ is nonempty and if $b_{1}, b_{2} \in \mathcal{A}^{ \pm 1}$ are the first, last, resp., letters of $U$ then $b_{1} \neq b_{2}^{-1}$ and $b_{1} \neq b_{2}$.

Lemma 5.1. Let $\Delta$ be a disk diagram over presentation (1.2) such that $\Delta$ has property $(A)$ and the word $W \equiv \varphi\left(\left.\partial\right|_{0} \Delta\right)$ has property (E). Then there exists a factorization $\left.\partial\right|_{0} \Delta=q_{1} q_{2}$ such that $\left|q_{1}\right|,\left|q_{2}\right|>0$ and $\left(q_{1}\right)_{+}=\left(q_{2}\right)_{-}=\alpha(0)$. In particular, $\varphi\left(q_{1}\right) \stackrel{\mathcal{G}_{2}}{=} 1, \varphi\left(q_{2}\right) \stackrel{\mathcal{G}_{2}}{=} 1$.

Proof. Let $\left.\partial\right|_{0} \Delta=e_{1} q e_{2}$, where $e_{1}, e_{2}$ are edges, $q$ is a subpath of $\left.\partial\right|_{0} \Delta$.

Suppose $e_{1}^{-1}$ is an edge of $\left.\partial\right|_{0} \Delta$. Since $e_{1}^{-1} \neq e_{2}$ by property (E) of $W$, it follows that $e_{1}^{-1}$ is an edge of $q$ and $q=r_{1} e_{1}^{-1} r_{2}$, where $r_{1}, r_{2}$ are subpaths of $q$. Hence, $q_{1}=e_{1} r_{1} e_{1}^{-1}$ and $q_{2}=r_{2} e_{2}$ are desired paths.

Hence, we may assume that $e_{1}^{-1}$ is an edge of the boundary path $\partial \Pi_{1}$ of a face $\Pi_{1}$. Arguing in a similar fashion, we may assume that $e_{2}^{-1}$ is an edge of the boundary path $\partial \Pi_{2}$ of a face $\Pi_{2}$. If $\Pi_{1}=\Pi_{2}$ then, in view of relations of the presentation (1.2), we have $\varphi\left(e_{1}\right)=\varphi\left(e_{2}\right)$ contrary to property $(\mathrm{E})$ of $W$. Hence, $\Pi_{1} \neq \Pi_{2}$.

Since for every edge $f$ of $\partial \Pi_{1}$ the edge $f^{-1}$ belongs to $\partial \Delta$ by property (A), we obtain the existence of a desired path $q_{1}$ of the form $q_{1}=e_{1}$ if $\left|\partial \Pi_{1}\right|=1$ or 
$q_{1}=e_{1} r f^{-1}$ if $\left|\partial \Pi_{1}\right|>1$, where $r$ is a subpath of $\left.\partial\right|_{0} \Delta, f$ is the edge of $\partial \Pi_{1}$ such that $e_{1}^{-1} f$ is a subpath of $\partial \Pi_{1}, f_{-}=\alpha(0)$, and $\left|q_{1}\right|<|\partial \Delta|$.

If $U \stackrel{\underline{G}_{2}}{=} 1$, let $\mu_{2}(U)$ denote the integer such that the precise word problem for presentation (1.2) holds for the pair $\left(U, \mu_{2}(U)\right)$. If $U \stackrel{\mathcal{G}_{2}}{\neq} 1$, we set $\mu_{2}(U):=\infty$.

Lemma 5.2. Let $U$ be a word such that $U \stackrel{\mathcal{G}_{2}}{=} 1$ and $U$ has property (E). Then there exists a factorization $U \equiv U_{1} U_{2}$ such that $\left|U_{1}\right|,\left|U_{2}\right|>0$ and

$$
\mu_{2}(U)=\mu_{2}\left(U_{1}\right)+\mu_{2}\left(U_{2}\right) .
$$

Proof. Consider a disk diagram $\Delta$ over (1.2) such that $\varphi\left(\left.\partial\right|_{0} \Delta\right) \equiv U$ and $|\Delta(2)|=$ $\mu_{2}(U)$. By Lemma 2.2(b), we may assume that $\Delta$ has property (A). Hence, it follows from Lemma 5.1 applied to $\Delta$ that there is a factorization $\left.\partial\right|_{0} \Delta=q_{1} q_{2}$ such that $\left|q_{1}\right|,\left|q_{2}\right|>0$ and $\left(q_{1}\right)_{+}=\left(q_{2}\right)_{-}=\alpha(0)$. Denote $U_{i}:=\varphi\left(q_{i}\right)$ and let $\Delta_{i}$ be the subdiagram of $\Delta$ bounded by $q_{i}, i=1,2$. Since $\Delta$ is a minimal disk diagram for $U$, it follows that $\Delta_{i}$ is a minimal disk diagram for $U_{i}, i=1,2$. Hence, $\left|\Delta_{i}(2)\right|=\mu_{2}\left(U_{i}\right)$ and

$$
\mu_{2}(U)=|\Delta(2)|=\left|\Delta_{1}(2)\right|+\left|\Delta_{2}(2)\right|=\mu_{2}\left(U_{1}\right)+\mu_{2}\left(U_{2}\right),
$$

as required.

Let $U$ be a nonempty word over $\mathcal{A}^{ \pm 1}$ and let $U(i, j)$, where $1 \leq i \leq|U|$ and $0 \leq j \leq|U|$, denote the subword of $U$ that starts with the $i$ th letter of $U$ and has length $j$. For example, $U=U(1,|U|)$ and $U(i, 0)$ is the empty subword.

If $a_{j} \in \mathcal{A}$, let $|U|_{a_{j}}$ denote the total number of occurrences of letters $a_{j}, a_{j}^{-1}$ in $U$. Note that we can decide whether $U \stackrel{\mathcal{G}_{2}}{=} 1$ in time $O\left(|U|^{2}\right)$ by cancelling subwords $a_{j}^{ \pm n_{j}}, a_{j}^{-1} a_{j}, a_{j} a_{j}^{-1}$, and checking whether the word obtained by a process of such cancellations is empty.

Let $W$ be a nonempty word over $\mathcal{A}^{ \pm 1}$. Define a parameterized word $W[i, j, k, \ell]$, where $1 \leq i \leq|W|, 0 \leq j \leq|W|, 1 \leq k \leq m$, and $\ell$ is an integer that satisfies $|\ell| \leq|W|_{a_{k}}-|W(i, j)|_{a_{k}}$ so that

$$
W[i, j, k, \ell]:=W(i, j) a_{k}^{\ell}
$$

and the word $W(i, j) a_{k}^{\ell}$ is not empty, i.e., $j+|\ell| \geq 1$.

Note that the total number of such parameterized words $W[i, j, k, \ell]$ is bounded by $O\left(|W|^{3}\right)$, here $m=|\mathcal{A}|$ is a constant as the presentation (1.2) is fixed. Let $\mathcal{S}_{2}(W)$ denote the set of all parameterized words $W[i, j, k, \ell]$. Elements $W[i, j, k, \ell]$ and $W\left[i^{\prime}, j^{\prime}, k^{\prime}, \ell^{\prime}\right]$ of $\mathcal{S}_{2}(W)$ are defined to be equal if and only if the quadruples $(i, j, k, \ell),\left(i^{\prime}, j^{\prime}, k^{\prime}, \ell^{\prime}\right)$ are equal. Hence, we wish to distinguish between elements of $\mathcal{S}_{2}(W)$ and actual words represented by elements of $\mathcal{S}_{2}(W)$. It is clear that we can have $W(i, j) a_{k}^{\ell} \equiv W\left(i^{\prime}, j^{\prime}\right) a_{k^{\prime}}^{\ell^{\prime}}$ when $W[i, j, k, \ell] \neq W\left[i^{\prime}, j^{\prime}, k^{\prime}, \ell^{\prime}\right]$.

If $U$ is the word represented by $W[i, j, k, \ell]$, i.e., $U \equiv W(i, j) a_{k}^{\ell}$, then we denote this by writing

$$
U \stackrel{\star}{=} W[i, j, k, \ell] .
$$

We introduce a partial order on the set $\mathcal{S}_{2}(W)$ by setting

$$
W\left[i^{\prime}, j^{\prime}, k^{\prime}, \ell^{\prime}\right] \prec W[i, j, k, \ell]
$$


if $j^{\prime}<j$ or $j^{\prime}=j$ and $\left|\ell^{\prime}\right|<|\ell|$. In other words, we partially order the set $\mathcal{S}_{2}(W)$ by using the lexicographical order on pairs $(j,|\ell|)$ associated with elements $W[i, j, k, \ell]$ of $\mathcal{S}_{2}(W)$.

Define

$$
\mu_{2}(W[i, j, k, \ell]):=\mu_{2}\left(W(i, j) a_{k}^{\ell}\right) .
$$

To compute the number $\mu_{2}(W)=\mu_{2}(W[1,|W|, 1,0])$ in polynomial time, we use the method of dynamic programming in which the parameter is $(j,|\ell|)$. In other words, we compute the number $\mu_{2}(W[i, j, k, \ell])$ by induction on parameter $(j,|\ell|)$. The base of induction (or initialization) for $j+|\ell|=1$ is obvious as $\mu_{2}(W[i, j, k, \ell])$ is 0 or 1 depending on the presentation (1.2).

To make the induction step, we assume that the numbers $\mu_{2}\left(W\left[i^{\prime}, j^{\prime}, k^{\prime}, \ell^{\prime}\right]\right)$ are already computed for all $W\left[i^{\prime}, j^{\prime}, k^{\prime}, \ell^{\prime}\right]$ whenever $\left(j^{\prime},\left|\ell^{\prime}\right|\right) \prec(j,|\ell|)$ and we compute the number $\mu_{2}(W[i, j, k, \ell])$.

If $W(i, j) a_{k}^{\ell} \stackrel{\mathcal{G}_{2}}{\neq} 1$, we set $\mu_{2}\left(W\left[i^{\prime}, j^{\prime}, k^{\prime}, \ell^{\prime}\right]\right):=\infty$.

Assume that $W(i, j) a_{k}^{\ell} \stackrel{\mathcal{G}_{2}}{=} 1$.

Suppose that the word $W(i, j) a_{k}^{\ell}$ has property (E). Consider all possible factorizations for $W(i, j) a_{k}^{\ell}$ of the form

$$
W(i, j) a_{k}^{\ell} \equiv U_{1} U_{2}
$$

where $\left|U_{1}\right|,\left|U_{2}\right| \geq 1$. Let us show that either of the words $U_{1}, U_{2}$ can be represented in a form $W\left[i^{\prime}, j^{\prime}, k^{\prime}, \ell^{\prime}\right]$ so that $W\left[i^{\prime}, j^{\prime}, k^{\prime}, \ell^{\prime}\right] \prec W[i, j, k, \ell]$.

First suppose $\left|U_{1}\right| \leq|W(i, j)|=j$. If $i+\left|U_{1}\right| \leq|W|$, we have

$$
U_{1} \stackrel{\star}{=} W\left[i,\left|U_{1}\right|, 1,0\right], \quad U_{2} \stackrel{\star}{=} W\left[i+\left|U_{1}\right|, j-\left|U_{1}\right|, k, \ell\right] .
$$

On the other hand, if $i+\left|U_{1}\right|=|W|+1$, we have

$$
U_{1} \stackrel{\star}{=} W\left[i,\left|U_{1}\right|, 1,0\right], \quad U_{2} \stackrel{\star}{=} W[|W|, 0, k, \ell] .
$$

Now suppose $\left|U_{1}\right|>|W(i, j)|=j$. Let $\ell_{1}, \ell_{2}$ be integers such that $\ell_{1}, \ell_{2}, \ell$ have the same sign, $\ell=\ell_{1}+\ell_{2}$, and $j+|\ell|=\left|U_{1}\right|+\ell_{2}$. Then we have

$$
U_{1} \stackrel{\star}{=} W\left[i,\left|U_{1}\right|, k, \ell_{1}\right], \quad U_{2} \stackrel{\star}{=} W\left[1,0, k, \ell_{2}\right] .
$$

Note that the induction parameter $\left(j^{\prime},\left|\ell^{\prime}\right|\right)$ for indicated representations of $U_{1}, U_{2}$ is smaller than the parameter $(j,|\ell|)$ for the original parameterized word $W[i, j, k, \ell]$. It follows from Lemma 5.2 applied to the word $W(i, j) a_{k}^{\ell}$ that there is a factorization

$$
W(i, j) a_{k}^{\ell} \equiv U_{1}^{\prime} U_{2}^{\prime}
$$

such that $\left|U_{1}^{\prime}\right|,\left|U_{2}^{\prime}\right| \geq 1$ and $\mu_{2}\left(W(i, j) a_{k}^{\ell}\right)=\mu_{2}\left(U_{1}^{\prime}\right)+\mu_{2}\left(U_{2}^{\prime}\right)$. Hence, taking the minimum

$$
\min \left(\mu_{2}\left(U_{1}\right)+\mu_{2}\left(U_{2}\right)\right)
$$

over all factorizations (5.2), we obtain the number $\mu_{2}\left(W(i, j) a_{k}^{\ell}\right)$.

Assume that the word $W(i, j) a_{k}^{\ell}$ has no property (E), i.e., $W(i, j) a_{k}^{\ell} \equiv b U b^{-1}$ or $W(i, j) a_{k}^{\ell} \equiv b$ or $W(i, j) a_{k}^{\ell} \equiv b U b$, where $b \in \mathcal{A}^{ \pm 1}$ and $U$ is a word.

First suppose that $W(i, j) a_{k}^{\ell} \equiv b U b^{-1}$. Note that the word $U$ can be represented in a form $W\left[i^{\prime}, j^{\prime}, k^{\prime}, \ell^{\prime}\right]$ so that $W\left[i^{\prime}, j^{\prime}, k^{\prime}, \ell^{\prime}\right] \prec W[i, j, k, \ell]$. Indeed, if $|\ell|=0$ then

$$
U \stackrel{\star}{=} W[i+1, j-2, k, 0] \text {. }
$$


On the other hand, if $|\ell|>0$ then

$$
U \stackrel{\star}{=} W\left[i+1, j-1, k, \ell_{1}\right],
$$

where $\left|\ell_{1}\right|=|\ell|-1$ and $\ell_{1} \ell \geq 0$. Hence, the number $\mu_{2}(U)$ is available by induction hypothesis. Since $\mu_{2}(W[i, j, k, \ell])=\mu_{2}(U)$, we obtain the required number $\mu_{2}(W[i, j, k, \ell])$.

In case $W(i, j) a_{k}^{\ell} \equiv b$, the number $\mu_{2}(W[i, j, k, \ell])$ is either one or $\infty$ depending on the presentation (1.2).

Finally, consider the case when $W(i, j) a_{k}^{\ell} \equiv b U b$. Denote $b=a_{k_{1}}^{\delta}$, where $a_{k_{1}} \in \mathcal{A}$ and $\delta= \pm 1$.

If $j=0$, i.e., $W(i, j) a_{k}^{\ell} \equiv a_{k}^{\ell}$, then $k=k_{1}$ and the number $\mu_{2}(W[i, j, k, \ell])$ can be easily computed in time $O(\log |W|)$ as $|\ell| \leq|W|$ and we can use binary representation for $\ell$.

Suppose $j>0$. If $\ell=0$ then $U b^{2} \stackrel{\star}{=} W\left[i+1, j-1, k_{1}, \delta\right]$. If $|\ell|>0$ then $k=k_{1}$, $\ell$ and $\delta$ have the same sign, and $U b^{2} \stackrel{\star}{=} W[i+1, j-1, k, \ell+\delta]$.

In either subcase $j=0$ or $j>0$, we obtain

$$
\begin{aligned}
& U b^{2} \stackrel{\star}{=} W\left[i^{\prime}, j^{\prime}, k^{\prime}, \ell^{\prime}\right], \quad W\left[i^{\prime}, j^{\prime}, k^{\prime}, \ell^{\prime}\right] \prec W[i, j, k, \ell], \\
& \mu_{2}(W[i, j, k, \ell])=\mu_{2}\left(W\left[i^{\prime}, j^{\prime}, k^{\prime}, \ell^{\prime}\right]\right) .
\end{aligned}
$$

This completes our inductive procedure of computation of numbers $\mu_{2}(W[i, j, k, \ell])$ for all $W[i, j, k, \ell] \in \mathcal{S}_{2}(W)$.

Since the length of every word $W(i, j) a_{k}^{\ell}$ is at most $|W|$, it follows that our computation of the number $\mu_{2}(W[i, j, k, \ell])$ can be done in time $O(|W| \log |W|)$ including additions of binary representations of numbers $\mu_{2}\left(U_{1}\right), \mu_{2}\left(U_{2}\right)$ to compute the minimum (5.3). Since the cardinality $\left|\mathcal{S}_{2}(W)\right|$ of the set $\mathcal{S}_{2}(W)$ is bounded by $O\left(|W|^{3}\right)$, we conclude that computation of numbers $\mu_{2}(W[i, j, k, \ell])$ for all words $W[i, j, k, \ell] \in \mathcal{S}_{2}(W)$ can be done in deterministic time $O\left(|W|^{4} \log |W|\right)$. This means that both the bounded word problem and the precise word problem for presentation (1.2) can be solved in time $O\left(|W|^{4} \log |W|\right)$.

The proof of Theorem 1.2 is complete.

Corollary 1.3. Let $W$ be a word over $\mathcal{A}^{ \pm 1}$ and $n \geq 0$ be an integer. Then the decision problems that inquire whether the width $h(W)$ or the spelling length $h_{1}(W)$ of $W$ is equal to $n$ belong to $L^{3}$ and $P$. Specifically, the problems can be solved in deterministic space $O\left((\log |W|)^{3}\right)$ or in deterministic time $O\left(|W|^{4} \log |W|\right)$.

Proof. Observe that the decision problem asking whether the width (resp. the spelling length) of a word $W$ is $n$ is equivalent to the precise word problem whose input is $(W, n)$ for presentation (1.2) in which $E_{i}=\mathbb{N}$ (resp. $E_{i}=\{1\}$ ) for every $i=1, \ldots, m$. Therefore, a reference to proven Theorem 1.2 shows that the problem asking whether the width (resp. the spelling length) of $W$ is $n$ belongs to both $\mathrm{L}^{3}$ and $\mathrm{P}$ and yields the required space and time bounds for this problem.

\section{Calculus of Brackets for Group Presentation (1.4)}

As in Theorem 1.4 consider a group presentation

$$
\mathcal{G}_{3}=\left\langle a_{1}, a_{2}, \ldots, a_{m} \| a_{2} a_{1}^{n_{1}} a_{2}^{-1}=a_{1}^{n_{2}}\right\rangle,
$$

where $n_{1}, n_{2}$ are nonzero integers. Suppose $W$ is a nonempty word over $\mathcal{A}^{ \pm 1}$ such

that $W \stackrel{\underline{G}_{3}}{=} 1, n \geq 0$ is an integer and $W$ is a product of at most $n$ conjugates 
of the words $\left(a_{2} a_{1}^{n_{1}} a_{2}^{-1} a_{1}^{-n_{2}}\right)^{ \pm 1}$. Then, as follows from arguments of the proof of Lemma 2.1, there exists a disk diagram $\Delta$ over presentation (1.4) such that $\varphi\left(\left.\partial\right|_{0} \Delta\right) \equiv W$ and $|\Delta(2)| \leq n$. Without loss of generality, we may assume that $\Delta$ is reduced. Consider the word $\rho_{a_{1}}(W)$ over $\mathcal{A}^{ \pm 1} \backslash\left\{a_{1}, a_{1}^{-1}\right\}$ which is obtained from $W$ by erasing all occurrences of $a_{1}^{ \pm 1}$ letters.

An oriented edge $e$ of $\Delta$ is called an $a_{i}$-edge if $\varphi(e)=a_{i}^{ \pm 1}$. We also consider a tree $\rho_{a_{1}}(\Delta)$ obtained from $\Delta$ by contraction of all $a_{1}$-edges of $\Delta$ into points and subsequent identification of all pairs of edges $e, f$ such that $e_{-}=f_{-}$and $e_{+}=f_{+}$. If an edge $e^{\prime}$ of $\rho_{a_{1}}(\Delta)$ is obtained from an edge $e$ of $\Delta$ this way, we set $\varphi\left(e^{\prime}\right):=\varphi(e)$. It is easy to check that this definition is correct. This labeling function turns the tree $\rho_{a_{1}}(\Delta)$ into a disk diagram over presentation $\left\langle a_{2}, \ldots, a_{m} \| \varnothing\right\rangle$.

Two faces $\Pi_{1}, \Pi_{2}$ in a disk diagram $\Delta$ over (1.4) are termed related, denoted $\Pi_{1} \leftrightarrow \Pi_{2}$, if there is an $a_{2}$-edge $e$ such that $e \in \partial \Pi_{1}$ and $e^{-1} \in \partial \Pi_{2}$. Consider the minimal equivalence relation $\sim_{2}$ on the set of faces of $\Delta$ generated by the relation $\leftrightarrow$. An $a_{i}$-band, where $i>1$, is a minimal subcomplex $\Gamma$ of $\Delta$ ( $\Gamma$ is not necessarily simply connected) that contains an $a_{i}$-edge $f$, and, if there is a face $\Pi$ in $\Delta$ with $f \in(\partial \Pi)^{ \pm 1}$, then $\Gamma$ must contain all faces of the equivalence class $[\Pi]_{\sim_{2}}$ of $\Pi$. Hence, an $a_{i}$-band $\Gamma$ is either a subcomplex consisting of a single nonoriented edge, denoted $\left\{f, f^{-1}\right\}$, where $f$ is an $a_{i}$-edge, $i>1$, and $f, f^{-1} \in \partial \Delta$, or $\Gamma$ consists of all faces of an equivalence class $[\Pi]_{\sim_{2}}$ when $i=2$. Clearly, the latter case is possible only if $i=2$. In this latter case, if $\Gamma$ contains faces but $\Gamma$ has no face in $[\Pi]_{\sim_{2}}$ whose boundary contains an $a_{2}$-edge $f$ with $f^{-1} \in \partial \Delta, \Gamma$ is called a closed $a_{2}$-band.

Lemma 6.1. Suppose that $\Delta$ is a reduced disk diagram over presentation (1.4). Then there are no closed $a_{2}$-bands in $\Delta$ and every $a_{i}$-band $\Gamma$ of $\Delta, i>1$, is a disk subdiagram of $\Delta$ such that

$$
\left.\partial\right|_{\left(f_{1}\right)_{-}} \Gamma=f_{1} s_{1} f_{2} s_{2}
$$

where $f_{1}, f_{2}$ are edges of $\partial \Delta$ with $\varphi\left(f_{1}\right)=\varphi\left(f_{2}\right)^{-1}=a_{i}, s_{1}, s_{2}$ are simple paths with $\varphi\left(s_{1}\right) \equiv a_{1}^{k n_{1}}, \varphi\left(s_{2}\right) \equiv a_{1}^{-k n_{2}}$ for some integer $k \geq 0$, $\Gamma$ contains $k$ faces, and $\partial \Gamma$ is a simple closed path when $k>0$.

Proof. Since $\Delta$ is reduced, it follows that if two faces $\Pi_{1}, \Pi_{2}$ are related by the relation $\sim$, then $\Pi_{1}$ is not a mirror copy of $\Pi_{2}$, i.e., $\varphi\left(\Pi_{1}\right) \not \equiv \varphi\left(\Pi_{2}\right)^{-1}$.

Assume that there is a closed $a_{2}$-band $\Gamma_{0}$ in $\Delta$. Then it follows from the above remark that there is a disk subdiagram $\Delta_{0}$ of $\Delta$, surrounded by $\Gamma_{0}$, such that $\varphi\left(\Delta_{0}\right) \equiv a_{1}^{k_{0} n_{i}}$, where $i=1$ or $i=2$ and $k_{0}=\left|\Gamma_{0}(2)\right|>0$. This, however, is a contradiction to the Magnus's Freiheitssatz, see [18, [19. Recall that the Magnus's Freiheitssatz for a one-relator group presentation

$$
\mathcal{G}_{0}=\langle\mathcal{A} \| R=1\rangle
$$

claims that every letter $a \in \mathcal{A}^{ \pm 1}$, that appears in a cyclically reduced word $R$ over $\mathcal{A}^{ \pm 1}$, will also appear in $W^{ \pm 1}$ whenever $W$ is reduced and $W \stackrel{\mathcal{G}_{0}}{=} 1$.

Hence, no $a_{2}$-band $\Gamma$ in $\Delta$ is closed and, therefore, $\partial \Gamma=f_{1} s_{1} f_{2} s_{2}$ as described in Lemma's statement. If $k>0$ and $\partial \Gamma$ is not a simple closed path, then a proper subpath of $\partial \Gamma$ bounds a disk subdiagram $\Delta_{1}$ such that $\varphi\left(\Delta_{1}\right)$ is a proper subword of $\varphi(\partial \Gamma) \equiv a_{2} a_{1}^{k n_{1}} a_{2}^{-1} a_{1}^{-k n_{2}}$, where $k>0$. Then it follows from Lemma 2.1 that either $a_{1}^{\ell} \stackrel{\mathcal{G}_{3}}{=} 1$, where $\ell \neq 0$, or $a_{1}^{\ell^{\prime}} a_{2}^{\varepsilon} \stackrel{\mathcal{G}_{3}}{=} 1$, where $\varepsilon= \pm 1$. The first equality contradicts Magnus's Freiheitssatz and the second one is impossible in the abelianization of $\mathcal{G}_{3}$. 
The factorization $\left.\partial\right|_{\left(f_{1}\right)_{-}} \Gamma=f_{1} s_{1} f_{2} s_{2}$ of Lemma 6.1] will be called the standard boundary of an $a_{i}$-band $\Gamma$, where $i>1$. Note that $\left|s_{1}\right|=\left|s_{2}\right|=0$ is the case when $k=0$, i.e., $\Gamma$ contains no faces and $\partial \Gamma=f_{1} f_{2}$ with $f_{2}^{-1}=f_{1}$.

An alternative way of construction of the tree $\rho_{a_{1}}(\Delta)$ from $\Delta$ can now be described as contraction of the paths $s_{1}, s_{2}$ of the standard boundary of every $a_{2}$-band $\Gamma$ of $\Delta$ into points, identification of the edges $f_{1}, f_{2}^{-1}$ of $(\partial \Gamma)^{ \pm 1}$, and contraction into points of all $a_{1}$-edges $e$ with $e \in \partial \Delta$.

Let $s$ be a path in a disk diagram $\Delta_{0}$ over presentation (1.4). We say that $s$ is a special arc of $\Delta_{0}$ if either $|s|=0$ or $|s|>0, s$ is a reduced simple path, and $s$ consists entirely of $a_{1}$-edges. In particular, if $s$ is a special arc then every subpath of $s, s^{-1}$ is also a special arc, and $s_{-} \neq s_{+}$whenever $|s|>0$.

Lemma 6.2. Suppose that $\Delta_{0}$ is a reduced disk diagram over presentation (1.4) and $s, t$ are special arcs in $\Delta_{0}$ such that $s_{+}=t_{-}$. Then there are factorizations $s=s_{1} s_{2}$ and $t=t_{1} t_{2}$ such that $s_{2}=t_{1}^{-1}$ and $s_{1} t_{2}$ is a special arc, see Fig. 6.1.

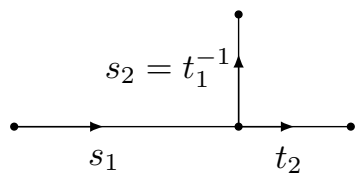

Fig. 6.1

Proof. First we observe that if $\Delta_{1}$ is a disk subdiagram of $\Delta_{0}$ such that every edge of $\partial \Delta_{1}$ is an $a_{1}$-edge, then it follows from Lemma 6.1 that $\Delta_{1}$ contains no faces, i.e., $\left|\Delta_{1}(2)\right|=0$, and so $\Delta_{1}$ is a tree.

We now prove this Lemma by induction on the length $|t| \geq 0$. If $|t|=0$ then our claim is true with $s_{1}=s$ and $t_{2}=t$. Assume that $|t|>0$ and $t=r e$, where $e$ is an edge. Since $r$ is also a special arc with $|r|<|t|$, the induction hypothesis applies to the pair $s, r$ and yields factorizations $s=s_{1}^{\prime} s_{2}^{\prime}$ and $r=r_{1} r_{2}$ such that $s_{2}^{\prime}=\left(r_{1}\right)^{-1}$ and $s_{1}^{\prime} r_{2}$ is a special arc. If $s_{1}^{\prime} r_{2} e$ is also a special arc, then the factorizations $s=s_{1}^{\prime} s_{2}^{\prime}$ and $t=t_{1} t_{2}$, where $t_{1}=r_{1}$ and $t_{2}=r_{2} e$, have the desired property and the induction step is complete.

Hence, we may assume that $s_{1}^{\prime} r_{2} e$ is not a special arc. Note that if $\left|r_{2}\right|>0$, then the path $s_{1}^{\prime} r_{2} e$ is reduced because $s_{1}^{\prime} r_{2}$ and $r_{2} e$ are special arcs. Assume that $s_{1}^{\prime} r_{2} e$ is not reduced. Then $\left|r_{2}\right|=0$ and the last edge of $s_{1}^{\prime}$ is $e^{-1}$. Denote $s_{1}^{\prime}=s_{11}^{\prime} e^{-1}$. Then, letting $s_{1}:=s_{11}^{\prime}, s_{2}:=t^{-1}$ and $t=t_{1} t_{2}$, where $t_{1}:=t$ and $\left|t_{2}\right|=0$, we have $s_{2}=t^{-1}$ and $s_{1} t_{2}$ is a desired special arc. Thus, we may assume that the path $s_{1}^{\prime} r_{2} e$ is reduced but not simple. Note that the path $s_{1}^{\prime} r_{2} e$ consists entirely of $a_{1}$-edges. Since $s_{1}^{\prime} r_{2}$ and $r_{2} e$ are simple, it follows that $e_{+}$belongs to $s_{1}^{\prime}$ and defines a factorization $s_{1}^{\prime}=s_{11} s_{12}$, where $\left|s_{12}\right|>0$. Hence, the path $s_{12} r_{2} e$ is a simple closed path which bounds a disk subdiagram $\Delta_{1}$ whose boundary $\partial \Delta_{1}$ consists of $a_{1}$-edges. By the observation made above, $\Delta_{1}$ is a tree, which, in view of the fact that $\partial \Delta_{1}$ is a simple closed path, implies that $\partial \Delta_{1}=e^{-1} e$. Therefore, $\left|r_{2}\right|=0$ and $s_{12}=e^{-1}$. This means that the path $s_{1}^{\prime} r_{2} e$ is not reduced, contrary to our assumption. This contradiction completes the proof.

Let $U$ be a word over $\mathcal{A}^{ \pm 1}$. If $a \in \mathcal{A}$ is a letter, then the a-length $|U|_{a}$ of $U$ is the number of occurrences of $a$ and $a^{-1}$ in $U$. We also define the complementary a-length by $|U|_{\bar{a}}:=|U|-|U|_{a}$. 
From now on we assume, unless stated otherwise, that $W$ is a nonempty word over $\mathcal{A}^{ \pm 1}$ such that $W \stackrel{\mathcal{G}_{3}}{=} 1$ and $\Delta$ is a reduced disk diagram over presentation (1.4) such that $\varphi\left(\left.\partial\right|_{0} \Delta\right) \equiv W$.

The definitions of the path $P_{W}$, of the map $\alpha: P_{W} \rightarrow \Delta$ and those of related notions, given in Sect. 2, are retained and are analogous to those used in Sects. 4-5.

Lemma 6.3. Suppose that $|W|_{\bar{a}_{1}}>2$. Then there exist vertices $v_{1}, v_{2} \in P_{W}$ such that $v_{1}<v_{2}$ and if $P_{W}\left(\right.$ fact, $\left.v_{1}, v_{2}\right)=p_{1} p_{2} p_{3}$, then the following hold true. There is a special arc $r$ in $\Delta$ such that $r_{-}=\alpha\left(v_{1}\right), r_{+}=\alpha\left(v_{2}\right), \varphi(r) \equiv a_{1}^{\ell}$ for some $\ell$, and

$$
\min \left(\left|\varphi\left(p_{2}\right)\right|_{\bar{a}_{1}},\left|\varphi\left(p_{1}\right)\right|_{\bar{a}_{1}}+\left|\varphi\left(p_{3}\right)\right|_{\bar{a}_{1}}\right) \geq \frac{1}{3}\left|\varphi\left(\left.\partial\right|_{0} \Delta\right)\right|_{\bar{a}_{1}}=\frac{1}{3}|W|_{\bar{a}_{1}} .
$$

In addition, if $|\vec{\Delta}(1)|_{a_{1}}:=|W|_{a_{1}}+\left(\left|n_{1}\right|+\left|n_{2}\right|\right)|\Delta(2)|$ denotes the number of $a_{1}$-edges of $\Delta$, then $|r| \leq \frac{1}{2}|\vec{\Delta}(1)|_{a_{1}}$.

Proof. Consider the tree $\rho_{a_{1}}(\Delta)$ constructed from $\Delta$ as above by collapsing $a_{1}$-edges $e$ into points and subsequent identification of edges $f, g$ with $f_{-}=g_{-}, f_{+}=g_{+}$. Then $\rho_{a_{1}}(\Delta)$ is a disk diagram over (1.4) with no faces and there is a surjective continuous cellular map

$$
\beta_{1}: \Delta \rightarrow \rho_{a_{1}}(\Delta)
$$

which preserves labels of edges $e$ with $\varphi(e) \neq a_{1}^{ \pm 1}$ and sends $a_{1}$-edges into vertices. It is also clear that if

$$
\alpha_{1}: P_{\rho_{a_{1}}(W)} \rightarrow \rho_{a_{1}}(\Delta)
$$

is the corresponding to the pair $\left(\rho_{a_{1}}(W), \rho_{a_{1}}(\Delta)\right)$ map, then there is a cellular continuous surjective map

$$
\beta: P_{W} \rightarrow P_{\rho_{a_{1}}(W)}
$$

which preserves labels of edges $e$ with $\varphi(e) \neq a_{1}^{ \pm 1}$, sends $a_{1}$-edges into vertices, and makes the following diagram commutative.

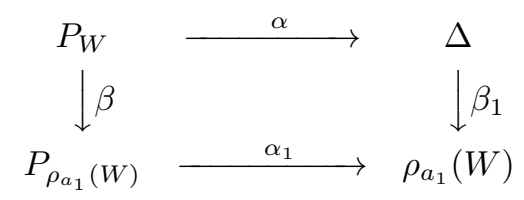

By Lemma 4.1 applied to $\rho_{a_{1}}(\Delta)$, there are vertices $u_{1}, u_{2} \in P_{\rho_{a_{1}}(W)}$ such that $\alpha_{1}\left(u_{1}\right)=\alpha_{1}\left(u_{2}\right)$ and if $P_{\rho_{a_{1}}(W)}\left(\right.$ fact, $\left.u_{1}, u_{2}\right)=s_{1} s_{2} s_{3}$, then

$$
\min \left(\left|s_{2}\right|,\left|s_{1}\right|+\left|s_{3}\right|\right) \geq \frac{1}{3}\left|\partial \rho_{a_{1}}(\Delta)\right|=\frac{1}{3}|W|_{\bar{a}_{1}} .
$$

It follows from definitions and Lemma 6.1 that there are some vertices $v_{1}, v_{2} \in P_{W}$ such that $\beta\left(v_{j}\right)=u_{j}, j=1,2$, the vertices $\alpha\left(v_{1}\right), \alpha\left(v_{2}\right)$ belong to $\partial \Gamma$, where $\Gamma$ is an $a_{i}$-band in $\Delta, i>1$, and the vertices $\alpha\left(v_{1}\right), \alpha\left(v_{2}\right)$ can be connected along $\partial \Gamma$ by a simple reduced path $r$ such that $\varphi(r) \equiv a_{1}^{\ell}$ for some integer $\ell$, where $\ell=0$ if $i>2$. Clearly, $r$ is a special arc of $\Delta$. Denote $P_{W}\left(\right.$ fact, $\left.v_{1}, v_{2}\right)=p_{1} p_{2} p_{3}$. Then it follows from definitions that $\left|\varphi\left(p_{i}\right)\right|_{\bar{a}_{1}}=\left|s_{i}\right|, i=1,2,3$, hence the inequality (6.1) yields that

$$
\min \left(\left|\varphi\left(p_{2}\right)\right|_{\bar{a}_{1}},\left|\varphi\left(p_{1}\right)\right|_{\bar{a}_{1}}+\left|\varphi\left(p_{3}\right)\right|_{\bar{a}_{1}}\right) \geq \frac{1}{3}\left|\partial \rho_{a_{1}}(\Delta)\right|=\frac{1}{3}|W|_{\bar{a}_{1}}
$$

as required. 
Furthermore, since $r$ is simple, reduced and $r_{-} \neq r_{+}$unless $|r|=0$, the path $r$ contains every $a_{1}$-edge $e$ of $\Delta$ at most once and if $r$ contains $e$ then $r$ contains no $e^{-1}$. Since the total number $|\vec{\Delta}(1)|_{a_{1}}$ of $a_{1}$-edges of $\Delta$ is equal to

$$
|\vec{\Delta}(1)|_{a_{1}}=|\varphi(\partial \Delta)|_{a_{1}}+\left(\left|n_{1}\right|+\left|n_{2}\right|\right)|\Delta(2)|,
$$

we obtain the desired inequality

$$
|r|=|\varphi(r)|_{a_{1}} \leq \frac{1}{2}|\vec{\Delta}(1)|_{a_{1}}=\frac{1}{2}|W|_{a_{1}}+\frac{1}{2}\left(\left|n_{1}\right|+\left|n_{2}\right|\right)|\Delta(2)| .
$$

A quadruple

$$
b=(b(1), b(2), b(3), b(4)),
$$

of integers $b(1), b(2), b(3), b(4)$ is called a bracket for the pair $(W, \Delta)$ if $b(1), b(2)$ satisfy the inequalities $0 \leq b(1) \leq b(2) \leq|W|$, and, in the notation

$$
P_{W}(\text { fact }, b(1), b(2))=p_{1} p_{2} p_{3},
$$

the following holds true. There exists a special arc $r(b)$ in $\Delta$ such that $r(b)_{-}=$ $\alpha(b(1)), r(b)_{+}=\alpha(b(2))$, and $\varphi(r(b)) \stackrel{0}{=} a_{1}^{b(3)}$. Furthermore, if $\Delta_{b}$ is the disk subdiagram of $\Delta$ defined by $\left.\partial\right|_{b(1)} \Delta_{b}=\alpha\left(p_{2}\right) r(b)^{-1}$ (such $\Delta_{b}$ is well defined as $r(b)$ is a special arc in $\Delta)$, then $\left|\Delta_{b}(2)\right|=b(4)$, see Fig. 6.2.

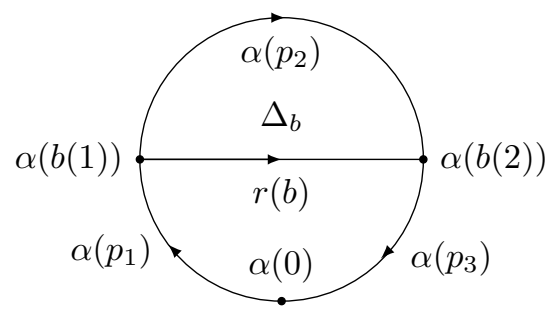

Fig. 6.2

This disk subdiagram $\Delta_{b}$ of $\Delta$, defined by $\left.\partial\right|_{b(1)} \Delta_{b}=\alpha\left(p_{2}\right) r(b)^{-1}$, is associated with the bracket $b$. The boundary subpath $\alpha\left(p_{2}\right)$ of $\Delta_{b}$ is denoted $a(b)$ and called the arc of the bracket $b$. The path $r(b)$ is termed the complementary arc of $b$.

For example, $b_{v}=(v, v, 0,0)$ is a bracket for every vertex $v$ of $P_{W}$, called a starting bracket at $v=b(1)$. Note that $a(b)=\alpha(v), r(b)=\alpha(v)$, and $\Delta_{b}=\{\alpha(v)\}$.

The final bracket for $(W, \Delta)$ is $c=(0,|W|, 0,|\Delta(2)|)$. Observe that $a(c)=\left.\partial\right|_{0} \Delta$, $r(c)=\{\alpha(0)\}$ and $\Delta_{c}=\Delta$.

Let $B$ be a finite set of brackets for the pair $(W, \Delta)$, perhaps, $B$ is empty. We say that $B$ is a bracket system for $(W, \Delta)$ if, for every pair $b, c \in B$ of distinct brackets, either $b(2) \leq c(1)$ or $c(2) \leq b(1)$.

Now we describe three kinds of elementary operations over a bracket system $B$ for the pair $(W, \Delta)$ : additions, extensions, and mergers.

Additions.

Suppose $b$ is a starting bracket, $b \notin B$, and $B \cup\{b\}$ is a bracket system. Then we may add $b$ to $B$ thus making an addition operation over $B$.

Extensions. 
Suppose $B$ is a bracket system, $b \in B$ is a bracket and $e_{1} a(b) e_{2}$ is a subpath of the boundary path $\left.\partial\right|_{0} \Delta$, where $a(b)$ is the arc of $b$ and $e_{1}, e_{2}$ are edges one of which could be missing.

Assume that $\varphi\left(e_{1}\right)=a_{1}^{\varepsilon}$, where $\varepsilon= \pm 1$. Since $e_{1}$ and $r(b)$ are special arcs of $\Delta$ and $e_{1} r(b)$ is a path, Lemma 6.2 applies and yields that either $e_{1} r(b)$ is a special arc or $r(b)=e_{1}^{-1} r_{1}$, where $r_{1}$ is a subpath of $r(b)$ and $r_{1}$ is a special arc. In the first case, define $r\left(b^{\prime}\right):=e_{1} r(b)$. In the second case, we set $r\left(b^{\prime}\right):=r_{1}$. Note that, in either case, $r\left(b^{\prime}\right)$ is a special arc and $\varphi\left(r\left(b^{\prime}\right)\right) \stackrel{0}{=} a_{1}^{b(3)+\varepsilon}$. In either case, we consider a bracket $b^{\prime}$ such that

$$
b^{\prime}(1)=b(1)-1, b^{\prime}(2)=b(2), b^{\prime}(3)=b(3)+\varepsilon, b^{\prime}(4)=b(4) .
$$

In either case, we have that $a\left(b^{\prime}\right)=e_{1} a(b), r\left(b^{\prime}\right)$ is defined as above, and $\Delta_{b^{\prime}}$ is the disk subdiagram whose boundary is $\partial \Delta_{b^{\prime}}=a\left(b^{\prime}\right) r\left(b^{\prime}\right)^{-1}$. We say that $b^{\prime}$ is obtained from $b$ by an extension of type 1 (on the left). If $(B \backslash\{b\}) \cup\left\{b^{\prime}\right\}$ is a bracket system, then replacement of $b \in B$ with $b^{\prime}$ in $B$ is called an extension operation over $B$ of type 1 .

Similarly, assume that $\varphi\left(e_{2}\right)=a_{1}^{\varepsilon}$, where $\varepsilon= \pm 1$. Since $r(b)$ is a simple path, it follows as above from Lemma 6.2 that either $r(b) e_{2}$ is a special arc or $r(b)=r_{2} e_{2}^{-1}$, where $r_{2}$ is a subpath of $r(b)$ and $r_{2}$ is a special arc. In the first case, define $r\left(b^{\prime}\right):=r(b) e_{2}$. In the second case, we set $r\left(b^{\prime}\right):=r_{2}$. Note that, in either case, $r\left(b^{\prime}\right)$ is a special arc and $\varphi\left(r\left(b^{\prime}\right)\right) \stackrel{0}{=} a_{1}^{b(3)+\varepsilon}$. In either case, we consider a bracket $b^{\prime}$ such that

$$
b^{\prime}(1)=b(1), b^{\prime}(2)=b(2)+1, b^{\prime}(3)=b(3)+\varepsilon, b^{\prime}(4)=b(4) .
$$

In either case, we have that $a\left(b^{\prime}\right)=a(b) e_{2}, r\left(b^{\prime}\right)$ is defined as above, and $\Delta_{b^{\prime}}$ is the disk subdiagram whose boundary is $\partial \Delta_{b^{\prime}}=a\left(b^{\prime}\right) r\left(b^{\prime}\right)^{-1}$. We say that $b^{\prime}$ is obtained from $b$ by an extension of type 1 (on the right). If $(B \backslash\{b\}) \cup\left\{b^{\prime}\right\}$ is a bracket system, then replacement of $b \in B$ with $b^{\prime}$ in $B$ is called an extension operation over $B$ of type 1 .

Now suppose that $\varphi\left(e_{1}\right)=\varphi\left(e_{2}\right)^{-1}=a_{2}^{\varepsilon}, \varepsilon= \pm 1$, and there is an $a_{2}$-bond $\Gamma$ whose standard boundary is $\left.\partial\right|_{\left(e_{i}\right)_{-}} \Gamma=e_{i} s_{i} e_{3-i} s_{3-i}$, where $i=1$ if $\varepsilon=1$ and $i=2$ if $\varepsilon=-1$. Recall that the standard boundary of an $a_{2}$-bond starts with an edge $f$ with $\varphi(f)=a_{2}$.

First we assume that $|b(3)| \neq 0$, i.e., $\varphi\left(s_{i}\right) \stackrel{0}{\neq} 1$. By Lemma 6.1, the paths $s_{1}, s_{2}$ are special arcs. Moreover, $\Gamma$ consists of $|b(3)| /\left|n_{i}\right|$ faces. Recall that $\Delta$ is reduced. It follows from Lemma 6.2 applied to special $\operatorname{arcs} r(b)$ and $s_{i}^{-1}$ that $r(b)=s_{i}$. Consider a bracket $b^{\prime}$ such that $b^{\prime}(1)=b(1)-1, b^{\prime}(2)=b(2)+1$, $b^{\prime}(3)=\left(\left|n_{1}^{-1} n_{2}\right|\right)^{\varepsilon} b(3)$, and $b^{\prime}(4)=b(4)+|b(3)| /\left|n_{i}\right|$. Note that $a\left(b^{\prime}\right)=e_{1} a(b) e_{2}$ and $r(b)=s_{i+1}^{-1}$. We say that $b^{\prime}$ is obtained from $b$ by an extension of type 2 . If $(B \backslash\{b\}) \cup\left\{b^{\prime}\right\}$ is a bracket system, then replacement of $b \in B$ with $b^{\prime}$ in $B$ is called an extension operation over $B$ of type 2 .

Assume that $e_{1}=e_{2}^{-1}$ and $\varphi\left(e_{1}\right) \neq a_{1}^{ \pm 1}$. Then we may consider a bracket $b^{\prime}$ such that $b^{\prime}(1)=b(1)-1, b^{\prime}(2)=b(2)+1, b^{\prime}(3)=b(3)$, and $b^{\prime}(4)=b(4)$. Since the path $a(b)$ is closed, it follows from Lemma 2.1 that $\varphi(a(b)) \stackrel{\mathcal{G}_{3}}{=} 1$ and so $b^{\prime}(3)=b(3)=0$ by Magnus's Freiheitssatz. Note that $a\left(b^{\prime}\right)=e_{1} a(b) e_{2}$ and $\left|r\left(b^{\prime}\right)\right|=0$. We say that $b^{\prime}$ is obtained from $b$ by an extension of type 3. If $(B \backslash\{b\}) \cup\left\{b^{\prime}\right\}$ is a bracket system, then replacement of $b \in B$ with $b^{\prime}$ in $B$ is called an extension operation over $B$ of type 3 . 
Mergers.

Suppose that $b_{1}, b_{2}$ are distinct brackets in $B$ such that $b_{1}(2)=b_{2}(1)$. Consider the disk diagram $\Delta_{b_{i}}$, associated with the bracket $b_{i}, i=1,2$, and let $\left.\partial\right|_{b_{i}(1)} \Delta_{b_{i}}=$ $a\left(b_{i}\right) r\left(b_{i}\right)^{-1}$ be the boundary of $\Delta_{b_{i}}$, where $a\left(b_{i}\right), r\left(b_{i}\right)$ are the arcs of $b_{i}, i=1,2$.

Since $r\left(b_{1}\right), r\left(b_{2}\right)$ are special arcs with $r\left(b_{1}\right)_{-}=r\left(b_{2}\right)_{+}$, it follows from Lemma 6.2 that there are factorizations $r\left(b_{1}\right)=r_{1} r_{0}, r\left(b_{2}\right)=r_{0}^{-1} r_{2}$ such that the path $r\left(b^{\prime}\right):=$ $r_{1} r_{2}$ is a special arc of $\Delta$. Note that $r\left(b^{\prime}\right)_{-}=\alpha\left(b_{1}(1)\right), r\left(b^{\prime}\right)_{+}=\alpha\left(b_{2}(2)\right)$, and the disk subdiagram $\Delta_{0}$ of $\Delta$ defined by $\partial \Delta_{0}=a\left(b_{1}\right) a\left(b_{2}\right) r\left(b^{\prime}\right)^{-1}$ contains $b_{1}(4)+b_{2}(4)$ faces. Therefore, we may consider a bracket $b^{\prime}$ such that $b^{\prime}(1)=b_{1}(1), b^{\prime}(2)=b_{2}(2)$, $b^{\prime}(3)=b_{1}(3)+b_{2}(3)$, and $b^{\prime}(4)=b_{1}(4)+b_{2}(4)$. Note that $a\left(b^{\prime}\right)=a\left(b_{1}\right) a\left(b_{2}\right)$, $r\left(b^{\prime}\right)=r_{1} r_{2}$, and $\Delta_{b^{\prime}}=\Delta_{0}$. We say that $b^{\prime}$ is obtained from $b_{1}, b_{2}$ by a merger operation. If $\left(B \backslash\left\{b_{1}, b_{2}\right\}\right) \cup\left\{b^{\prime}\right\}$ is a bracket system, then taking both $b_{1}, b_{2}$ out of $B$ and putting $b^{\prime}$ in $B$ is a merger operation over $B$.

We will say that additions, extensions, and mergers, as defined above, are elementary operations over brackets and bracket systems for $(W, \Delta)$.

Assume that one bracket system $B_{\ell}$ is obtained from another bracket system $B_{0}$ by a finite sequence $\Omega$ of elementary operations and $B_{0}, B_{1}, \ldots, B_{\ell}$ is the corresponding to $\Omega$ sequence of bracket systems. As before, such a sequence $B_{0}, B_{1}, \ldots, B_{\ell}$ of bracket systems is called operational. We say that the sequence $B_{0}, B_{1}, \ldots, B_{\ell}$ has size bounded by $\left(k_{1}, k_{2}\right)$ if $\ell \leq k_{1}$ and, for every $i$, the number of brackets in $B_{i}$ is at most $k_{2}$. Whenever it is not ambiguous, we also say that $\Omega$ has size bounded by $\left(k_{1}, k_{2}\right)$ if so does the corresponding to $\Omega$ sequence $B_{0}, B_{1}, \ldots, B_{\ell}$ of bracket systems.

We now study properties of brackets and bracket systems.

Lemma 6.4. If $b$ is a bracket for the pair $(W, \Delta)$, then $|b(3)| \leq \frac{1}{2}|\vec{\Delta}(1)|_{a_{1}}$.

Proof. By the definition of a bracket, the complementary arc $r(b)$ of $b$ is a special arc in $\Delta$, hence, either $|r(b)|=0$ or, otherwise, $|r(b)|>0$ and $r(b)$ is a simple, reduced path consisting entirely of $a_{1}$-edges. Since the total number of $a_{1}$-edges in $\Delta$ is $|\vec{\Delta}(1)|_{a_{1}}$, it follows that $|b(3)| \leq|r(b)| \leq \frac{1}{2}|\vec{\Delta}(1)|_{a_{1}}$.

Lemma 6.5. Suppose that $b, c$ are two brackets for the pair $(W, \Delta)$ and $b(1)=c(1)$, $b(2)=c(2)$. Then $b=c$.

Proof. Consider the complementary arcs $r(b), r(c)$ of brackets $b, c$, resp. Since $r(b)_{-}=r(c)_{-}, r(b)_{+}=r(c)_{+}$, and $r(b), r(c)^{-1}$ are special arcs of $\Delta$, it follows from Lemma 6.2 applied to special arcs $r(b), r(c)^{-1}$ that $r(b)=r(c)$. This means that $b(3)=c(3), \Delta_{b}=\Delta_{c}$ and $b(4)=c(4)$. Therefore, $b=c$, as desired.

Lemma 6.6. There exists a sequence of elementary operations that converts the empty bracket system for $(W, \Delta)$ into the final bracket system and has size bounded by $(4|W|,|W|)$.

Proof. For every $k$ with $0 \leq k \leq|W|$, consider a starting bracket $(k, k, 0,0)$ for $(W, \Delta)$. Making $|W|+1$ additions, we get a bracket system

$$
B_{W}=\{(k, k, 0,0)|0 \leq k \leq| W \mid\}
$$

of $|W|+1$ starting brackets. Now, looking at $\Delta$, we can easily find a sequence of extensions and mergers that converts $B_{W}$ into the final bracket system $B_{\ell}$. To estimate the total number of elementary operations, we note that the number of 
additions is $|W|+1$. The number of extensions is at most $|W|$ because every extension applied to a bracket system $B$ increases the number

$$
\eta(B):=\sum_{b \in B}(b(2)-b(1))
$$

by 1 or 2 and $\eta\left(B_{W}\right)=0$, while $\eta\left(B_{\ell}\right)=|W|$. The number of mergers is $|W|$ because the number of brackets $|B|$ in a bracket system $B$ decreases by 1 if $B$ is obtained by a merger and $\left|B_{W}\right|=|W|+1,\left|B_{\ell}\right|=1$. Hence, $\ell \leq 3|W|+1 \leq 4|W|$, as required.

Lemma 6.7. Suppose there is a sequence $\Omega$ of elementary operations that converts the empty bracket system $E$ for $(W, \Delta)$ into the final bracket system $F$ and has size bounded by $\left(k_{1}, k_{2}\right)$. Then there is also a sequence of elementary operations that changes $E$ into $F$ and has size bounded by $\left(7|W|, k_{2}\right)$.

Proof. Assume that the sequence $\Omega$ has an addition operation which introduces a starting bracket $c=(k, k, 0,0)$ with $0 \leq k \leq|W|$. Since the final bracket system contains no starting bracket, $c$ must disappear and an elementary operation is applied to $c$. If a merger is applied to $c$ and another bracket $b$ and the merger yields $\widehat{c}$, then $\widehat{c}=b$. This means that the addition of $c$ and the merger could be skipped without affecting the sequence otherwise. Note that the size of the new sequence $\Omega^{\prime}$ is bounded by $\left(k_{1}-2, k_{2}\right)$. Therefore, we may assume that no merger is applied to a starting bracket in $\Omega$. We also observe that, by the definition, an extension of type 2 is not applicable to a starting bracket (because of the condition $b(3) \neq 0)$.

Thus, by an obvious induction on $k_{1}$, we may assume that, for every starting bracket $c$ which is added by $\Omega$, an extension of type 1 or 3 is applied to $c$.

Now we will show that, for every starting bracket $c$ which is added by $\Omega$, there are at most 2 operations of addition of $c$ in $\Omega$. Arguing on the contrary, assume that $c_{1}, c_{2}, c_{3}$ are brackets equal to $c$ whose additions are used in $\Omega$. By the above remark, for every $i=1,2,3$, an extension of type 1 or 3 is applied to $c_{i}$, resulting in a bracket $\widehat{c}_{i}$.

Let $c_{1}, c_{2}, c_{3}$ be listed in the order in which the brackets $\widehat{c}_{1}, \widehat{c}_{2}, \widehat{c}_{3}$ are created by $\Omega$. Note that if $\widehat{c}_{1}$ is obtained from $c_{1}$ by an extension of type 3 , then

$$
\widehat{c}_{1}(1)=c_{1}(1)-1, \quad \widehat{c}_{1}(2)=c_{1}(2)+1 .
$$

This means that brackets $\widehat{c}_{2}, \widehat{c}_{3}$ could not be created by extensions after $\widehat{c}_{1}$ appears, as $b(2) \leq c(1)$ or $b(1) \geq c(2)$ for distinct brackets $b, c \in B$ of any bracket system $B$. This observation proves that $\widehat{c}_{1}$ is obtained from $c_{1}$ by an extension of type 1. Similarly to the forgoing argument, we can see that if $\widehat{c}_{1}$ is obtained by an extension of type 1 on the left/right, then $\widehat{c_{2}}$ must be obtained by an extension on the right/left, resp., and that $\widehat{c}_{3}$ cannot be obtained by any extension. This contradiction proves that it is not possible to have in $\Omega$ more than two additions of any starting bracket $c$. Thus, the number of additions in $\Omega$ is at most $2|W|+2$. As in the proof of Lemma 6.6. the number of extensions is $\leq|W|$ and the number of mergers is at most $2|W|+1$. Hence, the total number of elementary operations is at most $5|W|+3 \leq 7|W|$ as desired.

Lemma 6.8. Let there be a sequence $\Omega$ of elementary operations that transforms the empty bracket system for $(W, \Delta)$ into the final bracket system and has size bounded by $\left(k_{1}, k_{2}\right)$ and let $c$ be a starting bracket for $(W, \Delta)$. Then there is also 
a sequence of elementary operations that converts the bracket system $\{c\}$ into the final bracket system and has size bounded by $\left(k_{1}+1, k_{2}+1\right)$.

Proof. The proof is analogous to the proof of Lemma 4.5. The arguments in the cases when $c(1)=0$ or $c(1)=|W|$ are retained verbatim.

Assume that $0<c(1)<|W|$. As before, let $B_{0}, B_{1}, \ldots, B_{\ell}$ be the corresponding to $\Omega$ operational sequence of bracket systems, where $B_{0}$ is empty and $B_{\ell}$ is final. Let $B_{i^{*}}$ be the first bracket system of the sequence such that $B_{i^{*}} \cup\{c\}$ is not a bracket system. The existence of $B_{i^{*}}$ follows from the facts that $B_{0} \cup\{c\}$ is a bracket system and $B_{\ell} \cup\{c\}$ is not. Since $B_{0} \cup\{c\}$ is a bracket system, it follows that $i^{*} \geq 1$ and $B_{i^{*}-1} \cup\{c\}$ is a bracket system. Since $B_{i^{*}-1} \cup\{c\}$ is a bracket system and $B_{i^{*}} \cup\{c\}$ is not, there is a bracket $b \in B_{i^{*}}$ such that $b(1)<c(1)<b(2)$ and $b$ is obtained from a bracket $d_{1} \in B_{i^{*}-1}$ by an extension or $b$ is obtained from brackets $d_{1}, d_{2} \in B_{i^{*}-1}$ by a merger. In either case, it follows from definitions of elementary operations that $d_{j}(k)=c(1)$ for some $j, k \in\{1,2\}$. Hence, we can use a merger applied to $d_{j}$ and $c$ which would result in $d_{j}$, i.e., in elimination of $c$ from $B_{i^{*}-1} \cup\{c\}$ and in getting thereby $B_{i^{*}-1}$ from $B_{i^{*}-1} \cup\{c\}$. Now we can see that the original sequence of elementary operations, together with the merger $B_{i^{*}-1} \cup\{c\} \rightarrow B_{i^{*}-1}$ can be used to produce the following operational sequence of bracket systems

$$
B_{0} \cup\{c\}, \ldots, B_{i^{*}-1} \cup\{c\}, B_{i^{*}-1}, \ldots, B_{\ell} .
$$

Clearly, the size of this new sequence is bounded by $\left(k_{1}+1, k_{2}+1\right)$, as desired.

Lemma 6.9. Let $P_{W}=p_{1} p_{2}$, let the path $\alpha\left(p_{2}\right)$ be a special arc of $\Delta$ and let there exist a sequence $\Omega$ of elementary operations that transforms the empty bracket system for $(W, \Delta)$ into the final bracket system and has size bounded by $\left(\ell_{1}, \ell_{2}\right)$. Then there is also a sequence $\Omega_{p_{1}}$ of elementary operations such that $\Omega_{p_{1}}$ transforms the empty bracket system for $(W, \Delta)$ into the bracket system $\left\{\left(0,\left|p_{1}\right|,-k,|\Delta(2)|\right)\right\}$, where $\varphi\left(p_{2}\right) \stackrel{0}{=} a_{1}^{k}$, and $\Omega_{p_{1}}$ has size bounded by $\left(\ell_{1}, \ell_{2}\right)$. In addition, for every bracket $b \in B$, where $B$ is an intermediate bracket system of the corresponding to $\Omega_{p_{1}}$ operational sequence of bracket systems, it is true that $b(2) \leq\left|p_{1}\right|$.

Proof. Let $B_{0}, B_{1}, \ldots, B_{\ell}$ be the corresponding to $\Omega$ operational sequence of bracket systems, where $B_{0}$ is empty and $B_{\ell}=\{(0,|W|, 0,|\Delta(2)|)\}$ is the final bracket system for $(W, \Delta)$.

For every bracket system $B_{i}$, we will construct an associated bracket system $B_{i}^{\prime}$ as follows. Let $b \in B_{i}$ be a bracket. If $b(1)>\left|p_{1}\right|$, then we disregard $b$.

Suppose that $b(1) \leq\left|p_{1}\right|$ and $b(2)>\left|p_{1}\right|$. Let $p_{b}$ be a subpath of $p_{2}$ such that $\left(p_{b}\right)_{-}=\left(p_{2}\right)_{-},\left(p_{b}\right)_{+}=b(2)$ and let $\varphi\left(p_{b}\right) \stackrel{0}{=} a_{1}^{k_{p}}$, see Fig. 6.3. Since $r(b)$ and $\alpha\left(p_{b}\right)$ are special arcs in $\Delta$, it follows from Lemma 6.2 that the reduced path $r_{p}$, obtained from $r(b) \alpha\left(p_{b}\right)^{-1}$ by canceling pairs of edges of the form $e e^{-1}$, is a special arc such that

$$
\left(r_{p}\right)_{-}=\alpha(b(1)), \quad\left(r_{p}\right)_{+}=\alpha\left(\left(p_{b}\right)_{+}\right)=\alpha\left(\left|p_{1}\right|\right) .
$$

Hence, we can define a bracket

$$
b^{\prime}:=\left(b(1),\left|p_{1}\right|, b(3)-k_{p}, b(4)\right)
$$

whose complementary $\operatorname{arc} r\left(b^{\prime}\right)$ is $r_{p}, r\left(b^{\prime}\right):=r_{p}$. For every such $b \in B_{i}$, we include $b^{\prime}$ in $B_{i}^{\prime}$. 


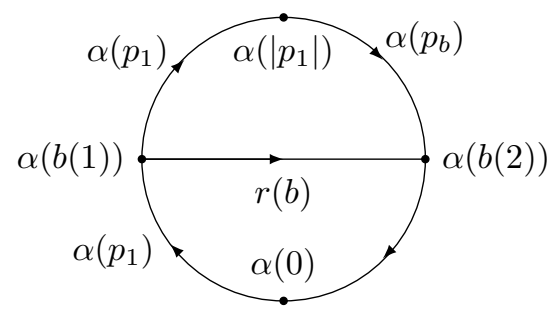

Fig. 6.3

If $b \in B_{i}$ satisfies $b(2) \leq\left|p_{1}\right|$, then we add $b$ to $B_{i}^{\prime}$.

Going over all brackets $b \in B_{i}$ as described above, we obtain a new bracket system $B_{i}^{\prime}$ associated with $B_{i}$. Observe that $B_{i+1}^{\prime}$ is either identical to $B_{i}^{\prime}$ or $B_{i+1}^{\prime}$ is obtained from $B_{i}^{\prime}$ by a single elementary operation which is identical to the elementary operation that is used to get $B_{i+1}$ from $B_{i}$.

Moreover, if $B_{i+1}^{\prime} \neq B_{i}^{\prime}$ and $B_{i+1}$ is obtained from $B_{i}$ by application of an elementary operation $\sigma$ to a bracket $b_{1} \in B_{i}$ or to brackets $b_{1}, b_{2} \in B_{i}$ (in the case when $\sigma$ is a merger) and this application results in $c$, written $c=\sigma\left(b_{1}\right)$ or $c=\sigma\left(b_{1}, b_{2}\right)$, then $B_{i+1}^{\prime}$ is obtained from $B_{i}^{\prime}$ by application of $\sigma^{\prime}$, where $\sigma^{\prime}$ has the same type as $\sigma$, to the bracket $b_{1}^{\prime} \in B_{i}^{\prime}$ or to the brackets $b_{1}^{\prime}, b_{2}^{\prime} \in B_{i}^{\prime}$ (in the case when $\sigma$ is a merger) and $c^{\prime}=\sigma^{\prime}\left(b_{1}^{\prime}\right)$ or $c^{\prime}=\sigma^{\prime}\left(b_{1}^{\prime}, b_{2}^{\prime}\right)$.

Indeed, this claim is immediate for additions, extensions of type 1 and mergers. On the other hand, it follows from the definitions of extensions of type 2 and 3 that an extension of type 2 or 3 can be applied only to a bracket $b_{1}$ with $b_{1}(2) \leq\left|p_{1}\right|$ and results in a bracket $c=\sigma\left(b_{1}\right)$ with $c(2) \leq\left|p_{1}\right|$. Hence, in this case, $b_{1}^{\prime}=b_{1}$, $c^{\prime}=c$, and our claim holds true again.

It follows from foregoing observations that a new sequence $B_{0}^{\prime}, B_{1}^{\prime}, \ldots, B_{\ell}^{\prime}$, when repetitions are disregarded, is operational, has size bounded by $\left(\ell_{1}, \ell_{2}\right), B_{0}^{\prime}$ is empty and $B_{\ell}^{\prime}$ consists of the single bracket $\left(0,\left|p_{1}\right|,-k,|\Delta(2)|\right)$, as desired. The last inequality $b(2) \leq\left|p_{1}\right|$ in lemma's statement is immediate from the definitions.

Lemma 6.10. Let $P_{W}=p_{1} p_{2} p_{3}$ be a factorization of the path $P_{W}$ such that $\alpha\left(p_{2}\right)$ is a special arc of $\Delta$ and let there exist a sequence of elementary operations of size bounded by $\left(\ell_{1}, \ell_{2}\right)$ that transforms the empty bracket system for $(W, \Delta)$ into the final bracket system. Then there is also a sequence of elementary operations that transforms a bracket system consisting of the single bracket $c_{0}:=\left(\left|p_{1}\right|,\left|p_{1} p_{2}\right|, k_{2}, 0\right)$, where $\varphi\left(p_{2}\right) \stackrel{0}{=} a_{1}^{k_{2}}$, into the final bracket system for $(W, \Delta)$ and has size bounded by $\left(\ell_{1}+1, \ell_{2}+1\right)$.

Proof. Consider a sequence $\Omega$ of elementary operations that transforms the empty bracket system for $(W, \Delta)$ into the final bracket system and has size bounded by $\left(\ell_{1}, \ell_{2}\right)$. Let $B_{0}, B_{1}, \ldots, B_{\ell}$ be the corresponding to $\Omega$ sequence of bracket systems, where $B_{0}$ is empty and $B_{\ell}=\{(0,|W|, 0,|\Delta(2)|)\}$ is the final bracket system for $(W, \Delta)$.

Define $i^{*}$ to be the minimal index so that $B_{i^{*}}$ contains a bracket $b$ such that $b(1) \leq\left|p_{1}\right|$ and $b(2) \geq\left|p_{1} p_{2}\right|$, i.e., the arc $a(b)$ of $b$ contains the path $\alpha\left(p_{2}\right)$. Since $B_{\ell}$ has this property and $B_{0}$ does not, such an $i^{*}$ exists and $0<i^{*} \leq \ell$.

First suppose that $\left|p_{2}\right|=0$. Then $c_{0}=\left(\left|p_{1}\right|,\left|p_{1}\right|, 0,0\right)$ and we can define the following operational sequence of bracket systems $B_{i}^{\prime}:=B_{i} \cup\left\{c_{0}\right\}$ for $i=0, \ldots, i^{*}-$ 
1. By the minimality of $i^{*}$, there is a unique bracket $b^{*} \in B_{i^{*}}$ such that $b^{*}(1)=\left|p_{1}\right|$ or $b^{*}(2)=\left|p_{1}\right|$.

Suppose $b^{*}(1)=b^{*}(2)=\left|p_{1}\right|$. Then $B_{i^{*}}$ is obtained from $B_{i^{*}-1}$ by addition of $c_{0}, B_{i^{*}}=B_{i^{*}-1} \cup\left\{c_{0}\right\}$, and we can consider the following operational sequence of bracket systems

$$
B_{0}^{\prime}=\left\{c_{0}\right\}, B_{1}^{\prime}, \ldots, B_{i^{*}-2}^{\prime}, B_{i^{*}-1}^{\prime}=B_{i^{*}}, B_{i^{*}+1}, \ldots, B_{\ell}
$$

that transforms $\left\{c_{0}\right\}$ into the final bracket system and has size bounded by $\left(\ell_{1}-\right.$ $\left.1, \ell_{2}+1\right)$.

Suppose $b^{*}(1)<b^{*}(2)$. Then a merger of $b^{*}$ and $c_{0}$ yields $b^{*}$, hence this merger turns $B_{i^{*}}^{\prime}=B_{i^{*}} \cup\left\{c_{0}\right\}$ into $B_{i^{*}}$. Now we can continue the original sequence $\Omega$ of elementary operations to get $B_{\ell}$. Thus the following

$$
B_{0}^{\prime}=\left\{c_{0}\right\}, B_{1}^{\prime}, \ldots, B_{i^{*}}^{\prime}, B_{i^{*}}, B_{i^{*}+1}, \ldots, B_{\ell}
$$

is an operational sequence of bracket systems that transforms $\left\{c_{0}\right\}$ into the final bracket system and has size bounded by $\left(\ell_{1}+1, \ell_{2}+1\right)$.

Now assume that $\left|p_{2}\right|>0$. For every bracket system $B_{i}$, where $i<i^{*}$, we construct an associated bracket system $B_{i}^{\prime}$ in the following manner.

Let $b \in B_{i}$ be a bracket. If $b(1) \geq\left|p_{1}\right|$ and $b(2) \leq\left|p_{1} p_{2}\right|$ and $b(1)-\left|p_{1}\right|+\left|p_{1} p_{2}\right|-$ $b(2)>0$, i.e., the arc $a(b)$ of $b$ is a proper subpath of the path $\alpha\left(p_{2}\right)$, then we disregard $b$.

Suppose that $b(1) \leq\left|p_{1}\right|$ and $\left|p_{1}\right|<b(2) \leq\left|p_{1} p_{2}\right|$, i.e., the arc $a(b)$ overlaps with a prefix subpath of the path $\alpha\left(p_{2}\right)$ of positive length. Let $p_{21}$ denote a subpath of $p_{2}$ given by $\left(p_{21}\right)_{-}=\left(p_{2}\right)_{-}$and $\left(p_{21}\right)_{+}=b(2)$, see Fig. 6.4. Then it follows from Lemma 6.2 applied to special arcs $r(b), \alpha\left(p_{21}\right)^{-1}$ of $\Delta$ that the reduced path, obtained from $r(b) \alpha\left(p_{21}\right)^{-1}$ by canceling pairs of edges of the form $e e^{-1}$, is a special $\operatorname{arcs}$ of $\Delta$. Therefore, we may consider a bracket

$$
b^{\prime}=\left(b(1),\left|p_{1}\right|, b(3)-k_{21}, b(4)\right),
$$

where $\varphi\left(p_{21}\right) \stackrel{0}{=} a_{1}^{k_{21}}$. For every such a bracket $b \in B_{i}$, we include $b^{\prime}$ into $B_{i}^{\prime}$.

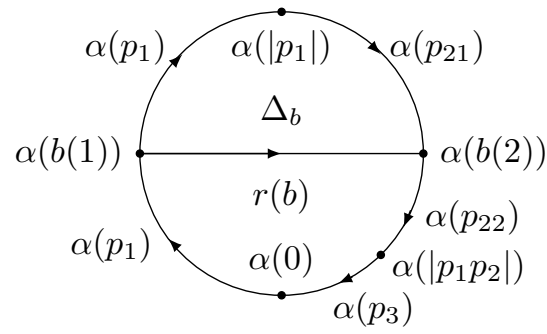

Fig. 6.4

Suppose $\left|p_{1}\right| \leq b(1)<\left|p_{1} p_{2}\right|$ and $\left|p_{1} p_{2}\right| \leq b(2)$, i.e., the arc $a(b)$ overlaps with a suffix of the path $\alpha\left(p_{2}\right)$ of positive length. Let $p_{22}$ denote a subpath of $p_{2}$ given by $\left(p_{22}\right)_{-}=b(1)$ and $\left(p_{22}\right)_{+}=\left(p_{2}\right)_{+}$, see Fig. 6.5. Then it follows from Lemma 6.2 applied to special arcs $\alpha\left(p_{22}\right)^{-1}, r(b)$ of $\Delta$ that the reduced path, obtained from $\alpha\left(p_{22}\right)^{-1} r(b)$ by canceling pairs of edges of the form $e e^{-1}$, is a special arcs of $\Delta$. Hence, we may consider a bracket

$$
b^{\prime}=\left(\left|p_{1} p_{2}\right|, b(2), b(3)-k_{22}, b(4)\right),
$$

where $\varphi\left(p_{22}\right) \stackrel{0}{=} a_{1}^{k_{22}}$. For every such a bracket $b \in B_{i}$, we include $b^{\prime}$ into $B_{i}^{\prime}$. 


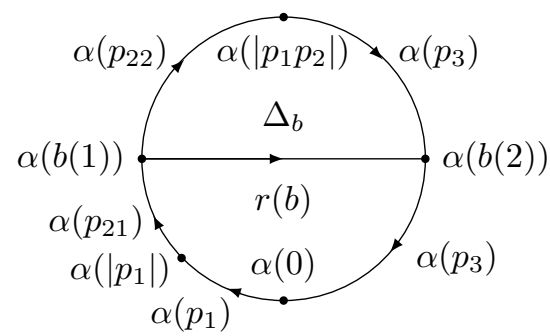

Fig. 6.5

If $b \in B_{i}$ satisfies either $b(2) \leq\left|p_{1}\right|$ or $b(1) \geq\left|p_{1} p_{2}\right|$, then we just add $b$ to $B_{i}^{\prime}$ without alterations.

We also put the bracket $c_{0}=\left(\left|p_{1}\right|,\left|p_{1} p_{2}\right|, k_{2}, 0\right)$ in every $B_{i}^{\prime}, i=0, \ldots, i^{*}-1$.

Going over all brackets $b \in B_{i}$, as described above, and adding $c_{0}$, we obtain a new bracket system $B_{i}^{\prime}$ associated with $B_{i}$.

Observe that for every $i<i^{*}-1$ the following holds true. Either $B_{i+1}^{\prime}$ is identical to $B_{i}^{\prime}$ or $B_{i+1}^{\prime}$ is obtained from $B_{i}^{\prime}$ by a single elementary operation which is identical to the elementary operation that is used to get $B_{i+1}$ from $B_{i}$. Moreover, if $B_{i+1}^{\prime} \neq B_{i}^{\prime}$ and $B_{i+1}$ is obtained from $B_{i}$ by application of an elementary operation $\sigma$ to a bracket $b_{1} \in B_{i}$ or to brackets $b_{1}, b_{2} \in B_{i}$ (in the case when $\sigma$ is a merger) and this application of $\sigma$ results in $c$, written $c=\sigma\left(b_{1}\right)$ or $c=\sigma\left(b_{1}, b_{2}\right)$, then $B_{i+1}^{\prime}$ is obtained from $B_{i}^{\prime}$ by application of $\sigma^{\prime}$, where $\sigma^{\prime}$ has the same type as $\sigma$, to the bracket $b_{1}^{\prime} \in B_{i}^{\prime}$ or to the brackets $b_{1}^{\prime}, b_{2}^{\prime} \in B_{i}^{\prime}$ (in the case when $\sigma$ is a merger) and $c^{\prime}=\sigma^{\prime}\left(b_{1}^{\prime}\right)$ or $c^{\prime}=\sigma^{\prime}\left(b_{1}^{\prime}, b_{2}^{\prime}\right)$, resp. Indeed, this claim is immediate for additions, extensions of type 1 and mergers. On the other hand, it follows from the definitions of extensions of types 2-3 and from the definition of $i^{*}$ that if $\sigma$ is an extension of type 2 or 3 then $\sigma$ can only be applied to a bracket $b_{1}$ such that $b_{1}(2) \leq\left|p_{1}\right|$ or $\left|p_{1} p_{2}\right| \leq b_{1}(1)$ and this application results in a bracket $c=\sigma\left(b_{1}\right)$ with $c(2) \leq\left|p_{1}\right|$ or $c(1) \leq\left|p_{1} p_{2}\right|$. Hence, in this case, $b_{1}^{\prime}=b_{1}, c^{\prime}=c$ and our claim holds true.

Since the bracket system $B_{i^{*}}$ contains a bracket $d$ such that $\alpha\left(p_{2}\right)$ is a subpath of $a(d)$ and $i^{*}$ is the minimal index with this property, it follows from the definition of elementary operations and from the facts that $\alpha\left(p_{2}\right)$ consists of $a_{1}$-edges and $\left|p_{2}\right|>0$ that either $d$ is obtained from a bracket $b_{1} \in B_{i^{*}-1}$ by an extension of type 1 or $d$ is obtained from brackets $b_{2}, b_{3} \in B_{i^{*}-1}$ by a merger.

First suppose that $d$ is obtained from a bracket $b_{1} \in B_{i^{*}-1}$ by an extension of type 1 . In this case, we pick the image $b_{1}^{\prime} \in B_{i^{*}-1}^{\prime}$ of $b_{1}$ and use a merger operation over $b_{1}^{\prime}, c_{0} \in B_{i^{*}-1}^{\prime}$ to get a bracket $c_{3}$. Let $B_{i^{*}}^{\prime}$ denote the new bracket system obtained from $B_{i^{*}-1}^{\prime}$ by the merger of $b_{1}^{\prime}, c_{0} \in B_{i^{*}-1}^{\prime}$, hence,

$$
B_{i^{*}}^{\prime}:=\left(B_{i^{*}-1}^{\prime} \backslash\left\{c_{0}, b_{1}^{\prime}\right\}\right) \cup\left\{c_{3}\right\} .
$$

Since $d$ is obtained from $b_{1}$ by an extension of type 1 , it follows that, for every $b \in B_{i^{*}-1}, b \neq b_{1}$, we have either $b(1) \geq d(2) \geq\left|p_{1} p_{2}\right|$ or $b(2) \leq d(1) \leq\left|p_{1}\right|$. Therefore, every bracket $b \in B_{i^{*}-1}, b \neq b_{1}$, has an image $b^{\prime} \in B_{i^{*}-1}^{\prime}$ and $b^{\prime}=b$. This, together with equalities $c_{3}(1)=d(1), c_{3}(2)=d(2)$ and Lemma 6.5, implies that $c_{3}=d$ and $B_{i^{*}}^{\prime}=B_{i^{*}}$. Thus we can consider the following sequence of bracket systems

$$
B_{0}^{\prime}, \ldots, B_{i^{*}-1}^{\prime}, B_{i^{*}}^{\prime}=B_{i^{*}}, B_{i^{*}+1}, \ldots, B_{\ell}
$$


that starts at $B_{0}^{\prime}=\left\{c_{0}\right\}$ and ends in the final bracket system $B_{\ell}$ for $(W, \Delta)$. It is clear that the size of this sequence is bounded by $\left(\ell_{1}, \ell_{2}+1\right)$. Hence, after deletion of possible repetitions in (6.3), we obtain a desired operational sequence.

Now assume that $d$ is obtained from brackets $b_{2}, b_{3} \in B_{i^{*}-1}$ by a merger and let $b_{2}(2)=b_{3}(1)$. To define $B_{i^{*}-\frac{1}{2}}^{\prime}$, we apply a merger operation to $b_{2}^{\prime}$ and $c_{0}$, which results in a bracket $c_{2}$. To define $B_{i^{*}}^{\prime}$, we apply a merger operation to $c_{2}$ and $b_{3}^{\prime}$ which results in a bracket $c_{3}$.

As above, we observe that since $d$ is obtained from $b_{2}, b_{3}$ by a merger, it follows that, for every bracket $b \in B_{i^{*}-1}, b \notin\left\{b_{1}, b_{2}\right\}$, we have either $b(1) \geq d(2) \geq\left|p_{1} p_{2}\right|$ or $b(2) \leq d(1) \leq\left|p_{1}\right|$. Therefore, every bracket $b \in B_{i^{*}-1}, b \notin\left\{b_{1}, b_{2}\right\}$, has an image $b^{\prime} \in B_{i^{*}-1}^{\prime}$ and $b^{\prime}=b$. This, together with equalities $c_{3}(1)=d(1), c_{3}(2)=d(2)$ and Lemma 6.5. implies that $c_{3}=d$ and $B_{i^{*}}^{\prime}=B_{i^{*}}$. Thus we can consider the following sequence of bracket systems

$$
B_{0}^{\prime}, \ldots, B_{i^{*}-1}^{\prime}, B_{i^{*}-\frac{1}{2}}^{\prime}, B_{i^{*}}^{\prime}=B_{i^{*}}, B_{i^{*}+1}, \ldots, B_{\ell}
$$

that starts at $B_{0}^{\prime}=\left\{c_{0}\right\}$ and ends in the final bracket system $B_{\ell}$. Clearly, the size of the sequence (6.3) is bounded by $\left(\ell_{1}+1, \ell_{2}+1\right)$. Hence, after deletion of possible repetitions in (6.3), we obtain a desired operational sequence.

Lemma 6.11. There exists a sequence of elementary operations that converts the empty bracket system for $(W, \Delta)$ into the final bracket system and has size bounded by

$$
\left(7|W|, C\left(\log |W|_{\bar{a}_{1}}+1\right)\right),
$$

where $C=\left(\log \frac{6}{5}\right)^{-1}$ and $\log |W|_{\bar{a}_{1}}:=0$ if $|W|_{\bar{a}_{1}}=0$.

Proof. The arguments of this proof are similar to those of the proof of Lemma 4.6 . Now we proceed by induction on $|W|_{\bar{a}_{1}} \geq 0$. To establish the basis for induction, we first check our claim in the case when $|W|_{\bar{a}_{1}} \leq 2$.

Let $|W|_{\bar{a}_{1}} \leq 2$. Then, looking at the tree $\rho_{a_{1}}(\Delta)$, we see that $|W|_{\bar{a}_{1}}=0$ or $|W|_{\bar{a}_{1}}=2$. If $|W|_{\bar{a}_{1}}=0$, then it follows from Lemma 6.1 applied to $\Delta$ that $\Delta$ is a tree consisting of $a_{1}$-edges and we can easily convert the empty bracket system for $(W, \Delta)$ into the final bracket system by using a single addition and $|W|$ extensions of type 1 . The size of the corresponding sequence is bounded by $(|W|+1,1)$ and the bound (6.4) holds as $C \geq 1$.

If now $|W|_{\bar{a}_{1}}=2$, then it follows from Lemma 6.1 applied to $\Delta$ that either $\Delta$ is a tree consisting of $a_{1}$-edges and two (oriented) $a_{i}$-edges, $i>1$, that form an $a_{i}$-band $\Gamma_{0}$ with no faces or $\Delta$ contains a single $a_{2}$-band $\Gamma$ that has faces and all edges of $\Delta$, that are not in $\Gamma$, are $a_{1}$-edges that form a forest whose trees are attached to $\Gamma$. In either case, we can convert the empty bracket system for $(W, \Delta)$ into the final bracket system by using a single addition and $\leq|W|$ extensions of type 1 and 3 if $\Delta$ is a tree or of type 1 and 2 if $\Delta$ is not a tree. The size of the corresponding sequence of elementary operations is bounded by $(|W|+1,1)$ and the bound (6.4) holds as $C \geq 1$. The base step is complete.

Making the induction step, assume $|W|_{\bar{a}_{1}}>2$. By Lemma 6.3 applied to $(W, \Delta)$, we obtain vertices $v_{1}, v_{2} \in P_{W}$ such that if $P_{W}\left(\right.$ fact, $\left.v_{1}, v_{2}\right)=p_{1} p_{2} p_{3}$ then there is a special arc $r$ in $\Delta$ such that $r_{-}=\alpha\left(v_{1}\right), r_{+}=\alpha\left(v_{2}\right)$ and

$$
\min \left(\left|\varphi\left(p_{2}\right)\right|_{\bar{a}_{1}},\left|\varphi\left(p_{1}\right)\right|_{\bar{a}_{1}}+\left|\varphi\left(p_{3}\right)\right|_{\bar{a}_{1}}\right) \geq \frac{1}{3}|W|_{\bar{a}_{1}},
$$


Let $\left.\partial\right|_{0} \Delta=q_{1} q_{2} q_{3}$, where $q_{i}=\alpha\left(p_{i}\right), i=1,2,3$. Consider disk subdiagrams $\Delta_{1}, \Delta_{2}$ of $\Delta$ given by $\left.\partial\right|_{v_{1}} \Delta_{2}=q_{2} r^{-1}$ and by $\left.\partial\right|_{0} \Delta_{1}=q_{1} r q_{3}$, see Fig. 6.6. Denote

$$
W_{2} \equiv \varphi\left(q_{2} r^{-1}\right), \quad W_{1} \equiv \varphi\left(q_{1} r q_{3}\right)
$$

and let $P_{W_{i}}=P_{W_{i}}\left(W_{i}, \Delta_{i}\right), i=1,2$, denote the corresponding paths such that $\alpha_{1}\left(P_{W_{1}}\right)=q_{1} r q_{3}$ and $\alpha_{2}\left(P_{W_{2}}\right)=q_{2} r^{-1}$. We also denote $\varphi(r) \stackrel{0}{=} a_{1}^{k_{r}}$.

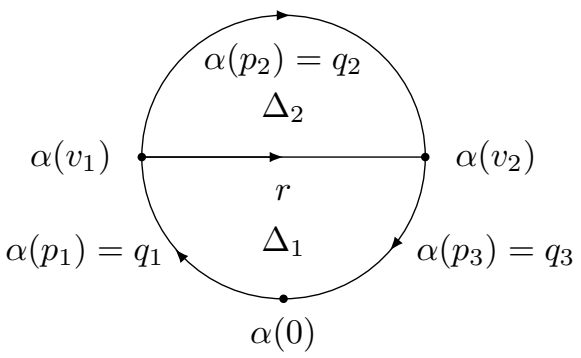

Fig. 6.6

Since $\left|W_{1}\right|_{\bar{a}_{1}},\left|W_{2}\right|_{\bar{a}_{1}}<|W|_{\bar{a}_{1}}$ by (6.5)), it follows from the induction hypothesis that there is a sequence $\widehat{\Omega}_{i}$ of elementary operations for $\left(W_{i}, \Delta_{i}\right)$ that transforms the empty bracket system into the final system and has size bounded by

$$
\left(7\left|W_{i}\right|, C\left(\log \left|W_{i}\right|_{\bar{a}_{1}}+1\right)\right),
$$

where $i=1,2$.

Furthermore, it follows from the bound (6.6) with $i=2$ and from Lemma 6.9 applied to $\Delta_{2}$ whose boundary label has factorization $\varphi\left(\left.\partial\right|_{0} \Delta_{2}\right) \equiv W_{2} \equiv W_{21} W_{22}$, where $W_{21} \equiv \varphi\left(p_{2}\right)$ and $W_{21} \equiv \varphi(r)^{-1}$, that there exists a sequence $\widehat{\Omega}_{2}$ of elementary operations for the pair $\left(W_{2}, \Delta_{2}\right)$ that transforms the empty bracket system $B_{2,0}$ into the bracket system $B_{2, \ell_{2}}=\left\{\left(0,\left|p_{2}\right|, k_{r},\left|\Delta_{2}(2)\right|\right)\right\}$ and has size bounded by

$$
\left(7\left|W_{2}\right|, C\left(\log \left|W_{2}\right|_{\bar{a}_{1}}+1\right)\right) .
$$

Let $B_{2,0}, B_{2,1}, \ldots, B_{2, \ell_{2}}$ denote the associated with $\widehat{\Omega}_{2}$ sequence of bracket systems.

It follows from the bound (6.6) with $i=1$ and from Lemma 6.10 applied to $\Delta_{1}$ whose boundary label has factorization $\varphi\left(\left.\partial\right|_{0} \Delta_{1}\right) \equiv W_{1} \equiv W_{11} W_{12} W_{13}$, where $W_{11} \equiv \varphi\left(p_{1}\right), W_{12} \equiv \varphi(r), W_{13} \equiv \varphi\left(p_{3}\right)$, that there exists a sequence $\Omega_{1}$ of elementary operations for $\left(W_{1}, \Delta_{1}\right)$ that transforms the bracket system $B_{1,0}=\left\{c_{r}\right\}$, where

$$
c_{r}:=\left(\left|p_{1}\right|,\left|p_{1}\right|+|r|, k_{r}, 0\right),
$$

into the final bracket system $B_{1, \ell_{1}}$ and has size bounded by

$$
\left(7\left|W_{1}\right|+1, C\left(\log \left|W_{1}\right|_{\bar{a}_{1}}+1\right)\right) .
$$

Let $B_{1,0}, B_{1,1}, \ldots, B_{1, \ell_{1}}$ be the associated with $\Omega_{1}$ sequence of bracket systems.

Now we will show that these two sequences $\Omega_{2}, \Omega_{1}$ of elementary operations, the first one for $\left(W_{2}, \Delta_{2}\right)$, and the second one for $\left(W_{1}, \Delta_{1}\right)$, could be modified and combined into a single sequence of elementary operations for $(W, \Delta)$ that converts the empty bracket system into the final bracket system and has size with a desired bound. 
Note that every bracket $b=(b(1), b(2), b(3), b(4)), b \in \cup_{j} B_{2, j}$, for $\left(W_{2}, \Delta_{2}\right)$, by Lemma 6.9, has the property that $b(2) \leq\left|p_{2}\right|$ and $b$ naturally gives rise to the bracket

$$
\widehat{b}:=\left(b(1)+\left|p_{1}\right|, b(2)+\left|p_{1}\right|, b(3), b(4)\right)
$$

for $(W, \Delta)$. Let $\widehat{B}_{2, j}$ denote the bracket system for $(W, \Delta)$ obtained from $B_{2, j}$ by replacing every bracket $b \in B_{2, j}$ with $\widehat{b}$. Then it is easy to verify that $\widehat{B}_{2,0}, \ldots, \widehat{B}_{2, \ell_{2}}$ is an operational sequence of bracket systems for $(W, \Delta)$ that changes the empty bracket system into

$$
\widehat{B}_{2, \ell_{2}}=\left\{\left(\left|p_{1}\right|,\left|p_{1}\right|+\left|p_{2}\right|, k_{r},\left|\Delta_{2}(2)\right|\right)\right\}
$$

and has size bounded by (6.7).

Consider a relation $\succ_{1}$ on the set of all pairs $(b, i)$, where $b \in B_{1, i}, i=0, \ldots, \ell_{1}$, defined so that $(c, i+1) \succ_{1}(b, i)$ if and only if $c \in B_{1, i+1}$ is obtained from brackets $b, b^{\prime} \in B_{1, i}$ by an elementary operation $\sigma$, here $b^{\prime}$ is missing if $\sigma$ is an extension.

Define a relation $\succeq$ on the set of all pairs $(b, i)$, where $b \in B_{1, i}, i=0, \ldots, \ell_{1}$, that is the reflexive and transitive closure of the relation $\succ_{1}$. Clearly, $\succeq$ is a partial order on the set of such pairs $(b, i)$ and if $\left(b_{2}, i_{2}\right) \succeq\left(b_{1}, i_{1}\right)$ then $i_{2} \geq i_{1}$ and

$$
b_{2}(1) \leq b_{1}(1) \leq b_{1}(2) \leq b_{2}(2) \text {. }
$$

Now we observe that every bracket $d=(d(1), d(2), d(3), d(4)), d \in B_{1, i}$, for $\left(W_{1}, \Delta_{1}\right)$ naturally gives rise to a bracket $\widehat{d}=(\widehat{d}(1), \widehat{d}(2), \widehat{d}(3), \widehat{d}(4))$ for $(W, \Delta)$ in the following manner.

If $(d, i)$ is not comparable with $\left(c_{r}, 0\right)$ by the relation $\succeq$ and $d(1) \leq\left|p_{1}\right|$, then

$$
\widehat{d}:=d \text {. }
$$

If $(d, i)$ is not comparable with $\left(c_{r}, 0\right)$ and $\left|p_{1}\right|+|r| \leq d(2)$, then

$$
\widehat{d}:=\left(d(1)+\left|p_{2}\right|-|r|, d(2)+\left|p_{2}\right|-|r|, d(3), d(4)\right) .
$$

If $(d, i) \succeq\left(c_{r}, 0\right)$, then

$$
\widehat{d}:=\left(d(1), d(2)+\left|p_{2}\right|-|r|, d(3), d(4)+\left|\Delta_{2}(2)\right|\right) .
$$

Note that the above three cases cover all possible situations.

As before, let $\widehat{B}_{1, i}:=\left\{\widehat{d} \mid d \in B_{1, i}\right\}$. Then it is easy to verify that $\widehat{B}_{1,0}, \ldots, \widehat{B}_{1, \ell_{1}}$ is an operational sequence of bracket systems for $(W, \Delta)$ that changes the bracket system

$$
\widehat{B}_{1,0}=\widehat{B}_{2, \ell_{2}}=\left\{\left(\left|p_{1}\right|,\left|p_{1}\right|+\left|p_{2}\right|, k_{r},\left|\Delta_{2}(2)\right|\right)\right\}
$$

into the final bracket system $\widehat{B}_{1, \ell_{1}}=\left\{\left(0,\left|p_{1}\right|+\left|p_{2}\right|+\left|p_{3}\right|, 0,\left|\Delta_{1}(2)\right|+\left|\Delta_{2}(2)\right|\right)\right\}$.

Thus, with the indicated changes, we can now combine the foregoing modified sequences of bracket systems for $\left(W_{2}, \Delta_{2}\right)$ and for $\left(W_{1}, \Delta_{1}\right)$ into a single operational sequence

$$
\widehat{B}_{2,0}, \ldots, \widehat{B}_{2, \ell_{2}}=\widehat{B}_{1,0}, \ldots, \widehat{B}_{1, \ell_{1}}
$$

of bracket systems for $(W, \Delta)$ that transforms the empty bracket system into the bracket system $\left\{\left(\left|p_{1}\right|,\left|p_{1}\right|+\left|p_{2}\right|, k_{r},\left|\Delta_{2}(2)\right|\right)\right\}$ and then continues to turn the latter into the final bracket system. It follows from definitions and bounds (6.7)-6.8) that the size of thus constructed sequence is bounded by

$$
\left(7\left|W_{1}\right|+7\left|W_{2}\right|+1, \max \left(C\left(\log \left|W_{1}\right|_{\bar{a}_{1}}+1\right)+1, C\left(\log \left|W_{2}\right|_{\bar{a}_{1}}+1\right)\right)\right)
$$


Therefore, in view of Lemma 6.7 it remains to show that

$$
\max \left(C\left(\log \left|W_{1}\right|_{\bar{a}_{1}}+1\right)+1, C\left(\log \left|W_{2}\right|_{\bar{a}_{1}}+1\right)\right) \leq C\left(\log |W|_{\bar{a}_{1}}+1\right) .
$$

In view of the inequality (6.5),

$$
\max \left(C\left(\log \left|W_{1}\right|_{\bar{a}_{1}}+1\right)+1, C\left(\log \left|W_{2}\right|_{\bar{a}_{1}}+1\right)\right) \leq C\left(\log \left(\frac{5}{6}|W|_{\bar{a}_{1}}\right)+1\right)+1,
$$

and $C\left(\log \left(\frac{5}{6}|W|_{\bar{a}_{1}}\right)+1\right)+1 \leq C\left(\log |W|_{\bar{a}_{1}}+1\right)$ for $C=\left(\log \frac{6}{5}\right)^{-1}$.

Let $W$ be an arbitrary nonempty word over the alphabet $\mathcal{A}^{ \pm 1}$, not necessarily representing the trivial element of the group given by presentation (1.4). As before, let $P_{W}$ be a simple labeled path with $\varphi\left(P_{W}\right) \equiv W$ and let vertices of $P_{W}$ be identified along $P_{W}$ with integers $0,1, \ldots,|W|$ so that $\left(P_{W}\right)_{-}=0, \ldots,\left(P_{W}\right)_{+}=$ $|W|$.

A quadruple

$$
b=(b(1), b(2), b(3), b(4))
$$

of integers $b(1), b(2), b(3), b(4)$ is called a pseudobracket for the word $W$ if $b(1), b(2)$ satisfy the inequalities $0 \leq b(1) \leq b(2) \leq|W|$ and $b(4) \geq 0$.

Let $p$ denote the subpath of $P_{W}$ with $p_{-}=b(1), p_{+}=b(2)$, perhaps, $p_{-}=p_{+}$ and $|p|=0$. The subpath $p$ of $P_{W}$ is denoted $a(b)$ and called the arc of the pseudobracket $b$.

For example, $b_{v}=(v, v, 0,0)$ is a pseudobracket for every vertex $v \in P_{W}$ and such $b_{v}$ is called a starting pseudobracket. Note that $a(b)=v=b(1)$. A final pseudobracket for $W$ is $c=(0,|W|, 0, k)$, where $k \geq 0$ is an integer. Note that $a(c)=P_{W}$.

Observe that if $b$ is a bracket for the pair $(W, \Delta)$, then $b$ is also a pseudobracket for the word $W$.

Let $B$ be a finite set of pseudobrackets for $W$, perhaps, $B$ is empty. We say that $B$ is a pseudobracket system if, for every pair $b, c \in B$ of distinct pseudobrackets, either $b(2) \leq c(1)$ or $c(2) \leq b(1)$. As before, $B$ is called a final pseudobracket system if $B$ contains a single pseudobracket which is final. Clearly, every bracket system for $(W, \Delta)$ is also a pseudobracket system for the word $W$.

Now we describe three kinds of elementary operations over pseudobrackets and over pseudobracket systems: additions, extensions, and mergers, which are analogous to those definitions for brackets and bracket systems, except there are no any diagrams and faces involved.

Let $B$ be a pseudobracket system for a word $W$.

\section{Additions.}

Suppose $b$ is a starting pseudobracket, $b \notin B$, and $B \cup\{b\}$ is a pseudobracket system. Then we can add $b$ to $B$ thus making an addition operation over $B$.

\section{Extensions.}

Suppose $B$ is a pseudobracket system, $b \in B$ is a pseudobracket and $e_{1} a(b) e_{2}$ is a subpath of $P_{W}$, where $a(b)$ is the arc of $b$ and $e_{1}, e_{2}$ are edges one of which could be missing.

Assume that $\varphi\left(e_{1}\right)=a_{1}^{\varepsilon_{1}}$, where $\varepsilon_{1}= \pm 1$. Then we consider a pseudobracket $b^{\prime}$ such that

$$
b^{\prime}(1)=b(1)-1, b^{\prime}(2)=b(2), b^{\prime}(3)=b(3)+\varepsilon_{1}, b^{\prime}(4)=b(4) .
$$


Note that $a\left(b^{\prime}\right)=e_{1} a(b)$. We say that $b^{\prime}$ is obtained from $b$ by an extension of type 1 (on the left). If $(B \backslash\{b\}) \cup\left\{b^{\prime}\right\}$ is a pseudobracket system, then replacement of $b \in B$ with $b^{\prime}$ in $B$ is called an extension operation over $B$ of type 1 .

Similarly, assume that $\varphi\left(e_{2}\right)=a_{1}^{\varepsilon_{2}}$, where $\varepsilon_{2}= \pm 1$. Then we consider a pseudobracket $b^{\prime}$ such that

$$
b^{\prime}(1)=b(1), b^{\prime}(2)=b(2)+1, b^{\prime}(3)=b(3)+\varepsilon_{2}, b^{\prime}(4)=b(4) .
$$

Note that $a\left(b^{\prime}\right)=a(b) e_{2}$. We say that $b^{\prime}$ is obtained from $b$ by an extension of type 1 (on the right). If $(B \backslash\{b\}) \cup\left\{b^{\prime}\right\}$ is a pseudobracket system, then replacement of $b \in B$ with $b^{\prime}$ in $B$ is called an extension operation over $B$ of type 1 .

Now suppose that both edges $e_{1}, e_{2}$ do exist and $\varphi\left(e_{1}\right)=\varphi\left(e_{2}\right)^{-1}=a_{2}^{\varepsilon}, \varepsilon= \pm 1$. We also assume that $b(3) \neq 0$ and that $b(3)$ is a multiple of $n_{1}$ if $\varepsilon=1$ or $b(3)$ is a multiple of $n_{2}$ if $\varepsilon=-1$. Consider a pseudobracket $b^{\prime}$ such that

$b^{\prime}(1)=b(1)-1, b^{\prime}(2)=b(2)+1, b^{\prime}(3)=\left(\left|n_{1}^{-1} n_{2}\right|\right)^{\varepsilon} b(3), b^{\prime}(4)=b(4)+|b(3)| /\left|n_{i(\varepsilon)}\right|$, where $i(\varepsilon)=1$ if $\varepsilon=1$ and $i(\varepsilon)=2$ if $\varepsilon=-1$. Note that $a\left(b^{\prime}\right)=e_{1} a(b) e_{2}$. We say that $b^{\prime}$ is obtained from $b$ by an extension of type 2 . If $(B \backslash\{b\}) \cup\left\{b^{\prime}\right\}$ is a pseudobracket system, then replacement of $b \in B$ with $b^{\prime}$ in $B$ is called an extension operation over $B$ of type 2 .

Assume that both $e_{1}, e_{2}$ do exist, $\varphi\left(e_{1}\right)=\varphi\left(e_{2}\right)^{-1}, \varphi\left(e_{1}\right) \neq a_{1}^{ \pm 1}$, and $b(3)=0$. Consider a pseudobracket $b^{\prime}$ such that

$$
b^{\prime}(1)=b(1)-1, b^{\prime}(2)=b(2)+1, b^{\prime}(3)=b(3), b^{\prime}(4)=b(4) .
$$

Note that $a\left(b^{\prime}\right)=e_{1} a(b) e_{2}$. We say that $b^{\prime}$ is obtained from $b$ by an extension of type 3. If $(B \backslash\{b\}) \cup\left\{b^{\prime}\right\}$ is a pseudobracket system, then replacement of $b \in B$ with $b^{\prime}$ in $B$ is called an extension operation over $B$ of type 3 .

Mergers.

Suppose that $b_{1}, b_{2}$ are distinct pseudobrackets in $B$ such that $b_{1}(2)=b_{2}(1)$. Consider a pseudobracket $b^{\prime}$ such that

$$
b^{\prime}(1)=b_{1}(1), b^{\prime}(2)=b_{2}(2), b^{\prime}(3)=b_{1}(3)+b_{2}(3), b^{\prime}(4)=b_{1}(4)+b_{2}(4) .
$$

Note that $a\left(b^{\prime}\right)=a\left(b_{1}\right) a\left(b_{2}\right)$. We say that $b^{\prime}$ is obtained from $b_{1}, b_{2}$ by a merger operation. Taking both $b_{1}, b_{2}$ out of $B$ and putting $b^{\prime}$ in $B$ is a merger operation over $B$.

We will say that additions, extensions, and mergers, as defined above, are elementary operations over pseudobrackets and pseudobracket systems for $W$.

Assume that one pseudobracket system $B_{\ell}$ is obtained from another pseudobracket system $B_{0}$ for $W$ by a finite sequence $\Omega$ of elementary operations and $B_{0}, B_{1}, \ldots, B_{\ell}$ is the corresponding to $\Omega$ sequence of pseudobracket systems. As above, we say that the sequence $B_{0}, B_{1}, \ldots, B_{\ell}$ is operational and that $B_{0}, B_{1}, \ldots, B_{\ell}$ has size bounded by $\left(k_{1}, k_{2}\right)$ if $\ell \leq k_{1}$ and, for every $i$, the number of pseudobrackets in $B_{i}$ is at most $k_{2}$. Whenever it is not ambiguous, we also say that $\Omega$ has size bounded by $\left(k_{1}, k_{2}\right)$ if so does the corresponding to $\Omega$ sequence $B_{0}, B_{1}, \ldots, B_{\ell}$ of pseudobracket systems.

Lemma 6.12. Suppose that the empty pseudobracket system $B_{0}$ for $W$ can be transformed by a finite sequence $\Omega$ of elementary operations into a final pseudobracket system $B_{\ell}=\{(0,|W|, 0, k)\}$. Then $W \stackrel{\mathcal{G}_{3}}{=} 1$ and there is a reduced disk diagram $\Delta$ over presentation (1.4) such that $\varphi\left(\left.\partial\right|_{0} \Delta\right) \equiv W$ and $|\Delta(2)| \leq k$. 
Proof. Let $B_{0}, B_{1}, \ldots, B_{\ell}$ be the corresponding to $\Omega$ sequence of pseudobracket systems, where $B_{0}$ is empty and $B_{\ell}$ is final. Consider the following claim.

(D1) If $c$ is a pseudobracket of $B_{i}, i=1, \ldots, \ell$, then $\varphi(a(c)) \stackrel{\mathcal{G}_{3}}{=} a_{1}^{c(3)}$, where $a(c)$ is the arc of $c$, and there is a disk diagram $\Delta_{c}$ over presentation (1.4) such that $\left.\partial\right|_{0} \Delta_{c}=s r^{-1}$, where $\varphi(s) \equiv \varphi(a(c)), r$ is simple, $\varphi(r) \equiv a_{1}^{c(3)}$ and $\left|\Delta_{c}(2)\right|=c(4)$.

Proof of Claim (D1). By induction on $i \geq 1$, we will prove that Claim (D1) holds for every pseudobracket $c \in B_{i}, i=1, \ldots, \ell$.

The base step of this induction is obvious since $B_{1}$ consists of a starting pseudobracket $c$ for which we have a disk diagram $\Delta_{c}$ consisting of a single vertex.

To make the induction step from $i$ to $i+1, i \geq 1$, we consider the cases corresponding to the type of the elementary operation that is used to get $B_{i+1}$ from $B_{i}$.

Suppose that $B_{i+1}$ is obtained from $B_{i}$ by an elementary operation $\sigma$ and $c \in$ $B_{i+1}$ is the pseudobracket obtained from $b_{1}, b_{2} \in B_{i}$ by application of $\sigma$, denoted $c=\sigma\left(b_{1}, b_{2}\right)$. Here one of $b_{1}, b_{2}$ or both, depending on type of $\sigma$, could be missing. By the induction hypothesis, Claim (D1) holds for every pseudobracket of $B_{i+1}$ different from $c$ and it suffices to show that Claim (D1) holds for $c$.

If $c \in B_{i+1}$ is obtained by an addition, then Claim (D1) holds for $c$ because it holds for any starting pseudobracket.

Let $c \in B_{i+1}$ be obtained from a pseudobracket $b \in B_{i}$ by an extension of type 1 on the left and $a(c)=e_{1} a(b)$, where $\varphi\left(e_{1}\right)=a_{1}^{\varepsilon_{1}}, \varepsilon_{1}= \pm 1$, and $a(c), a(b)$ are the arcs of $c, b$, resp. By the induction hypothesis applied to $b$, there is a disk diagram $\Delta_{b}$ over presentation (1.4) such that $\left.\partial\right|_{0} \Delta_{b}=s r^{-1}$, where $\varphi(s) \equiv \varphi(a(b)), r$ is simple, $\varphi(r) \equiv a_{1}^{b(3)}$, and $\left|\Delta_{b}(2)\right|=b(4)$. First we assume that the integers $\varepsilon_{1}, b(3)$ satisfy $\varepsilon_{1} b(3) \geq 0$. Then we consider a new "loose" edge $f$ such that $\varphi(f)=a_{1}^{\varepsilon_{1}}$. We attach the vertex $f_{+}$to the vertex $s_{-}$of $\Delta_{b}$ and obtain thereby a disk diagram $\Delta_{b}^{\prime}$ such that $\partial \Delta_{b}^{\prime}=f s r^{-1} f^{-1}$, see Fig. $6.7(\mathrm{a})$. Since $\varphi(a(c)) \equiv \varphi(f s) \equiv a_{1}^{\varepsilon_{1}} \varphi(a(b))$, $f r$ is simple and

$$
\varphi(f r) \equiv a_{1}^{\varepsilon_{1}+b(3)}, \quad\left|\Delta_{b}^{\prime}(2)\right|=\left|\Delta_{b}(2)\right|=b(4),
$$

it follows that we can use $\Delta_{b}^{\prime}$ as a required disk diagram $\Delta_{c}$ for $c$.

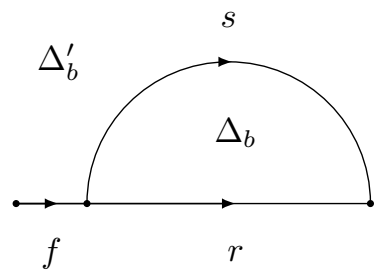

Fig. 6.7(a)

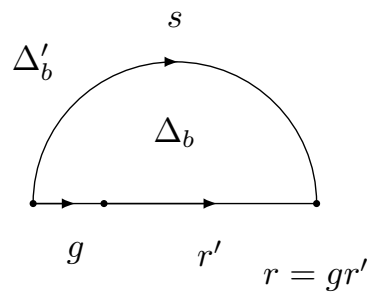

Fig. 6.7(b)

Now suppose that the integers $\varepsilon_{1}, b(3)$ satisfy $\varepsilon_{1} b(3)<0$, i.e., $b(3) \neq 0$ and $\varepsilon_{1}$, $b(3)$ have different signs. Then we can write $r=g r^{\prime}$, where $g$ is an edge of $r$ and $\varphi(g)=a_{1}^{-\varepsilon_{1}}$. Let $\Delta_{b}^{\prime}$ denote the diagram $\Delta_{b}^{\prime}$ whose boundary has factorization $\left.\partial\right|_{0} \Delta_{b}^{\prime}=\left(g^{-1} s\right)\left(r^{\prime}\right)^{-1}$, see Fig. 6.7(b). Note that $\varphi(a(c)) \equiv \varphi\left(g^{-1} s\right), r^{\prime}$ is simple, 
and

$$
\varphi\left(r^{\prime}\right) \equiv a_{1}^{\varepsilon_{1}+b(3)}, \quad\left|\Delta_{b}^{\prime}(2)\right|=\left|\Delta_{b}(2)\right|=b(4) .
$$

Hence, it follows that we can use $\Delta_{b}^{\prime}$ as a desired diagram $\Delta_{c}$ for $c$. The case of an extension of type 1 on the right is similar.

Let $c \in B_{i+1}$ be obtained from a pseudobracket $b \in B_{i}$ by an extension of type 2 and assume that $a(c)=e_{1} a(b) e_{2}$, where $\varphi\left(e_{1}\right)=\varphi\left(e_{2}\right)^{-1}=a_{2}$ (the subcase when $\varphi\left(e_{1}\right)=\varphi\left(e_{2}\right)^{-1}=a_{2}^{-1}$ is similar). Let $a(c), a(b)$ denote the arcs of pseudobrackets $c, b$, resp. According to the definition of an extension of type $2, b(3)$ is a multiple of $n_{1}$, say, $b(3)=n_{1} \ell$ and $\ell \neq 0$. By the induction hypothesis, there is a disk diagram $\Delta_{b}$ over presentation (1.4) such that $\left.\partial\right|_{0} \Delta_{b}=s r^{-1}$, where $\varphi(s) \equiv \varphi(a(b)), r$ is simple, $\varphi(r) \equiv a_{1}^{b(3)}$, and $\left|\Delta_{b}(2)\right|=b(4)$. Since $b(3)=n_{1} \ell$, there is a disk diagram $\Gamma$ over (1.4) such that

$$
\partial \Gamma=f_{1} s_{1} f_{2} s_{2}, \varphi\left(f_{1}\right)=a_{2}, \varphi\left(f_{2}\right)=a_{2}^{-1}, \varphi\left(s_{1}\right) \equiv a_{1}^{b(3)}, \varphi\left(s_{2}^{-1}\right) \equiv a_{1}^{b(3) n_{2} / n_{1}},
$$

and $\Gamma$ consists of $|\ell|>0$ faces. Note that $\Gamma$ itself is an $a_{2}$-band with $|\ell|$ faces. Attaching $\Gamma$ to $\Delta_{b}$ by identification of the paths $s_{1}$ and $r$ that have identical labels, we obtain a disk diagram $\Delta_{b}^{\prime}$ such that $\left.\partial\right|_{0} \Delta_{b}^{\prime}=f_{1} s f_{2} s_{2}$ and $\left|\Delta_{b}^{\prime}(2)\right|=\left|\Delta_{b}(2)\right|+|\ell|$, see Fig. 6.8. Then $\varphi(a(c)) \equiv \varphi\left(f_{1} s f_{2}\right), s_{2}^{-1}$ is simple, $\varphi\left(s_{2}^{-1}\right) \equiv a_{1}^{b(3) n_{2} / n_{1}}$ and we see that $\Delta_{b}^{\prime}$ satisfies all desired conditions for $\Delta_{c}$.

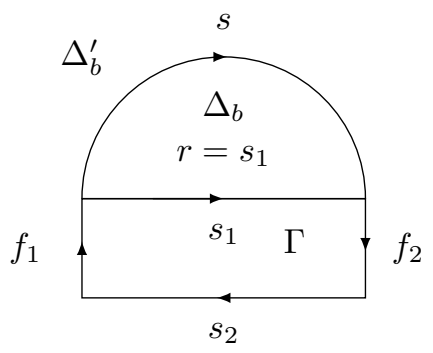

Fig. 6.8

Now let $c \in B_{i+1}$ be obtained from a pseudobracket $b \in B_{i}$ by an extension of type 3 and let $a(c)=e_{1} a(b) e_{2}$, where $\varphi\left(e_{1}\right)=\varphi\left(e_{2}\right)^{-1}, \varphi\left(e_{1}\right)=a_{j}^{\varepsilon}, \varepsilon= \pm 1$ and $j>1$. By the definition of an extension of type 3 , we have $b(3)=0$. Hence, by the induction hypothesis, there is a disk diagram $\Delta_{b}$ over (1.4) such that $\left.\partial\right|_{0} \Delta_{b}=s r^{-1}$, where $\varphi(s) \equiv \varphi(a(b)),|r|=0$ and $\left|\Delta_{b}(2)\right|=b(4)$. Consider a new "loose" edge $f$ such that $\varphi(f)=a_{j}^{\varepsilon}$. We attach the vertex $f_{+}$to the vertex $s_{-}=s_{+}$of $\Delta_{b}$ and obtain thereby a disk diagram $\Delta_{b}^{\prime}$ such that $\partial \Delta_{b}^{\prime}=f s f^{-1} r^{\prime}$, where $\left|r^{\prime}\right|=0$, see Fig. 6.9. Since $\varphi(a(c)) \equiv \varphi\left(f s f^{-1}\right)$ and $\left|\Delta_{b}^{\prime}(2)\right|=\left|\Delta_{b}(2)\right|=b(4)$, it follows that we can use $\Delta_{b}^{\prime}$ as a desired diagram $\Delta_{c}$ for $c$.

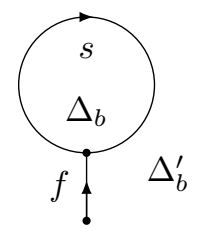

Fig. 6.9 
Let $c \in B_{i+1}$ be obtained from pseudobrackets $b_{1}, b_{2} \in B_{i}$ by a merger and $a(c)=a\left(b_{1}\right) a\left(b_{2}\right)$, where $a(c), a\left(b_{1}\right), a\left(b_{2}\right)$ are the arcs of the pseudobrackets $c, b_{1}, b_{2}$, resp.

By the induction hypothesis, there are disk diagrams $\Delta_{b_{1}}, \Delta_{b_{2}}$ over presentation (1.4) such that

$$
\left.\partial\right|_{0} \Delta_{b_{j}}=s_{j} r_{j}^{-1}, \varphi\left(s_{j}\right) \equiv \varphi\left(a\left(b_{j}\right)\right), \varphi\left(r_{j}\right) \equiv a_{1}^{b_{j}(3)},\left|\Delta_{b_{j}}(2)\right|=b_{j}(4),
$$

and $r_{j}$ is a simple path for $j=1,2$. Assume that the numbers $b_{1}(3), b_{2}(3)$ satisfy the condition $b_{1}(3) b_{2}(3) \geq 0$. We attach $\Delta_{b_{1}}$ to $\Delta_{b_{2}}$ by identification of the vertices $\left(s_{1}\right)_{+}$and $\left(s_{2}\right)_{-}$and obtain thereby a disk diagram $\Delta_{b}^{\prime}$ such that $\partial \Delta_{b}^{\prime}=s_{1} s_{2}\left(r_{1} r_{2}\right)^{-1}$ and $\left|\Delta_{b}^{\prime}(2)\right|=\left|\Delta_{b_{1}}(2)\right|+\left|\Delta_{b_{2}}(2)\right|$, see Fig. 6.10(a). Note that $\varphi(a(c)) \equiv \varphi\left(s_{1} s_{2}\right), r_{1} r_{2}$ is a simple path and $\varphi\left(r_{1} r_{2}\right) \equiv a_{1}^{b_{1}(3)+b_{2}(3)}$. Hence, $\Delta_{b}^{\prime}$ is a desired disk diagram $\Delta_{c}$ for $c$.

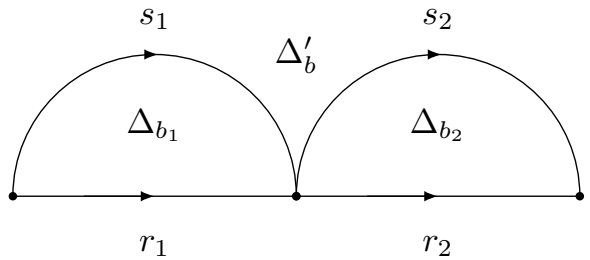

Fig. 6.10(a)

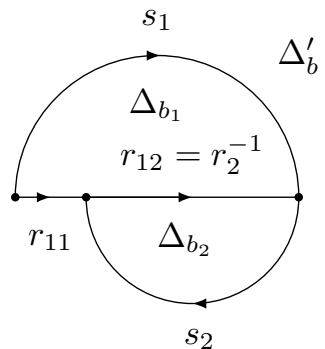

Fig. 6.10(b)

Now suppose that $b_{1}(3), b_{2}(3)$ satisfy the condition $b_{1}(3) b_{2}(3)<0$. For definiteness, assume that $\left|b_{1}(3)\right| \geq\left|b_{2}(3)\right|$ (the case $\left|b_{1}(3)\right| \leq\left|b_{2}(3)\right|$ is analogous). Denote $r_{1}=r_{11} r_{12}$, where $\left|r_{12}\right|=\left|r_{2}\right|=\left|b_{2}(3)\right|$. Then we attach $\Delta_{b_{2}}$ to $\Delta_{b_{1}}$ by identification of the paths $r_{12}$ and $r_{2}^{-1}$ that have identical labels equal to $a_{1}^{-b_{2}(3)}$, see Fig. 6.10(b). Doing this produces a disk diagram $\Delta_{b}^{\prime}$ such that $\partial \Delta_{b}^{\prime}=s_{1} s_{2} r_{11}^{-1}$ and $\left|\Delta_{b}^{\prime}(2)\right|=\left|\Delta_{b_{1}}(2)\right|+\left|\Delta_{b_{2}}(2)\right|$. Note that $\varphi(a(c)) \equiv \varphi\left(s_{1} s_{2}\right), r_{11}$ is a simple path and $\varphi\left(r_{11}\right) \equiv a_{1}^{b_{1}(3)+b_{2}(3)}$. Hence $\Delta_{b}^{\prime}$ is a desired disk diagram $\Delta_{c}$ for $c$. The induction step is complete and Claim (D1) is proven.

To finish the proof of Lemma 6.12, we note that it follows from Claim (D1) applied to the pseudobracket $b_{F}=(0,|W|, 0, k)$ of the final system $B_{\ell}=\left\{b_{F}\right\}$ that there is a disk diagram $\Delta_{b_{F}}$ such that $\left.\partial\right|_{0} \Delta_{b_{F}}=s r^{-1}$, where $\varphi(s) \equiv W,|r|=0$ and $\left|\Delta_{b_{F}}(2)\right| \leq k$. It remains to pick a reduced disk diagram $\Delta$ with these properties of $\Delta_{b_{F}}$.

Lemma 6.13. Suppose $W$ is a nonempty word over $\mathcal{A}^{ \pm 1}$ and $n \geq 0$ is an integer. Then $W$ is a product of at most $n$ conjugates of the words $\left(a_{2} a_{1}^{n_{1}} a_{2}^{-1} a_{1}^{-n_{2}}\right)^{ \pm 1}$ if and only if there is a sequence $\Omega$ of elementary operations such that $\Omega$ transforms the empty pseudobracket system for $W$ into a final pseudobracket system $\left\{\left(0,|W|, 0, n^{\prime}\right)\right\}$, where $n^{\prime} \leq n, \Omega$ has size bounded by

$$
\left(7|W|, C\left(\log |W|_{\bar{a}_{1}}+1\right)\right),
$$


where $C=\left(\log \frac{6}{5}\right)^{-1}$, and, if $b$ is a pseudobracket of an intermediate pseudobracket system of the corresponding to $\Omega$ sequence of pseudobracket systems, then

$$
|b(3)| \leq \frac{1}{2}\left(|W|_{a_{1}}+\left(\left|n_{1}\right|+\left|n_{2}\right|\right) n\right) .
$$

Proof. Assume that $W$ is a product of at most $n$ conjugates of the words

$$
\left(a_{2} a_{1}^{n_{1}} a_{2}^{-1} a_{1}^{-n_{2}}\right)^{ \pm 1} \text {. }
$$

It follows from Lemma 2.1 that there is a reduced disk diagram $\Delta$ over presentation (1.4) such that $\varphi\left(\left.\partial\right|_{0} \Delta\right) \equiv W$ and $|\Delta(2)| \leq n$. Applying Lemma 6.11 to the pair $(W, \Delta)$, we obtain a sequence of elementary operations over bracket systems for $(W, \Delta)$ that converts the empty bracket system for $(W, \Delta)$ into the final bracket system $\{(0,|W|, 0,|\Delta(2)|)\}$ and has size bounded by $\left(7|W|, C\left(\log |W|_{\bar{a}_{1}}+1\right)\right)$. Since every bracket $b$ and every bracket system $B$ for $(W, \Delta)$ could be considered as a pseudobracket and a pseudobracket system for $W$, resp., we automatically have a desired sequence of pseudobracket systems. The inequality (6.9) follows from the definition of a bracket for $(W, \Delta)$ and the inequalities

$$
|b(3)| \leq \frac{1}{2}|\vec{\Delta}(1)|_{a_{1}}=\frac{1}{2}\left(|W|_{a_{1}}+\left(\left|n_{1}\right|+\left|n_{2}\right|\right)|\Delta(2)|\right), \quad|\Delta(2)| \leq n .
$$

Conversely, the existence of a sequence of elementary operations over pseudobracket systems for $W$, as specified in Lemma 6.13, implies, by virtue of Lemma 6.12, that $W \stackrel{\mathcal{G}_{3}}{=} 1$ and that there exists a reduced disk diagram $\Delta$ such that $\varphi\left(\left.\partial\right|_{0} \Delta\right) \equiv W$ and $|\Delta(2)|=n^{\prime} \leq n$. Hence, $W$ is a product of $n^{\prime} \leq n$ conjugates of words $\left(a_{2} a_{1}^{n_{1}} a_{2}^{-1} a_{1}^{-n_{2}}\right)^{ \pm 1}$, as required.

\section{Proof of Theorem 1.4}

Theorem 1.4. Let the group $\mathcal{G}_{3}$ be defined by a presentation of the form

$$
\mathcal{G}_{3}:=\left\langle a_{1}, \ldots, a_{m} \| a_{2} a_{1}^{n_{1}} a_{2}^{-1}=a_{1}^{n_{2}}\right\rangle,
$$

where $n_{1}, n_{2}$ are some nonzero integers. Then both the bounded and precise word problems for (1.4) are in $L^{3}$ and in P. Specifically, the problems can be solved in deterministic space $O\left(\left(\max (\log |W|, \log n)(\log |W|)^{2}\right)\right.$ or in deterministic time $O\left(|W|^{4}\right)$.

Proof. As in the proof of Theorem 1.2, we start with $\mathrm{L}^{3}$ part of Theorem 1.4. First we discuss a nondeterministic algorithm which solves the bounded word problem for presentation (1.4) and which is now based on Lemma 6.13.

Given an input $\left(W, 1^{n}\right)$, where $W$ is a nonempty word (not necessarily reduced) over the alphabet $\mathcal{A}^{ \pm 1}$ and $n \geq 0$ is an integer, written in unary notation as $1^{n}$, we begin with the empty pseudobracket system and nondeterministically apply a sequence of elementary operations of size $\leq\left(7|W|, C\left(\log |W|_{\bar{a}_{1}}+1\right)\right)$, where $C=\left(\log \frac{6}{5}\right)^{-1}$. If such a sequence of elementary operations results in a final pseudobracket system, consisting of a single pseudobracket $\left\{\left(0,|W|, 0, n^{\prime}\right)\right\}$, where $n^{\prime} \leq n$, then our algorithm accepts and, in view of Lemma 6.13, we may conclude that $W$ is a product of $n^{\prime} \leq n$ conjugates of the words $\left(a_{2} a_{1}^{n_{1}} a_{2}^{-1} a_{1}^{-n_{2}}\right)^{ \pm 1}$. It follows from definitions and Lemma 6.13 that the number of elementary operations needed for this algorithm to accept is at most $7|W|$. Hence, it follows from the definition of elementary operations over pseudobracket systems for $W$ that the time needed to run this nondeterministic algorithm is bounded by $O(|W|)$. To estimate the space requirements of this algorithm, we note that if $b$ is a pseudobracket for $W$, then 
$b(1), b(2)$ are integers in the range from 0 to $|W|$, hence, when written in binary notation, will take at most $C^{\prime}(\log |W|+1)$ space, where $C^{\prime}$ is a constant. Since $b(3), b(4)$ are also integers that satisfy inequalities

$$
\begin{aligned}
& |b(3)| \leq \frac{1}{2}\left(|W|_{a_{1}}+\left(\left|n_{1}\right|+\left|n_{2}\right|\right) n\right) \leq|W|\left(\left|n_{1}\right|+\left|n_{2}\right|\right)(n+1), \\
& 0 \leq b(4) \leq n
\end{aligned}
$$

and $\left|n_{1}\right|+\left|n_{2}\right|$ is a constant, it follows that the total space required to run this algorithm is at most

$$
\begin{aligned}
& \left(2 C^{\prime}(\log |W|+1)+C^{\prime} \log \left(|W|\left(\left|n_{1}\right|+\left|n_{2}\right|\right)(n+1)\right)\right) \cdot C\left(\log |W|_{\bar{a}_{1}}+1\right)+ \\
& +\left(C^{\prime} \log (n+1)\right) \cdot C\left(\log |W|_{\bar{a}_{1}}+1\right)=O((\max (\log |W|, \log n) \log |W|) .
\end{aligned}
$$

As in the proof of Theorem 1.2, it now follows from Savitch's theorem 38, see also [3, 32, that there is a deterministic algorithm that solves the bounded word problem for presentation (1.4) in space $O\left(\max (\log |W|, \log n)(\log |W|)^{2}\right)$.

To solve the precise word problem for presentation (1.4), assume that we are given a pair $\left(W, 1^{n}\right)$ and we wish to find out if $W$ is a product of $n$ conjugates of the words $\left(a_{2} a_{1}^{n_{1}} a_{2}^{-1} a_{1}^{-n_{2}}\right)^{ \pm 1}$ and $n$ is minimal with this property. Using the foregoing deterministic algorithm, we check whether the bounded word problem is solvable for the two pairs $\left(W, 1^{n-1}\right)$ and $\left(W, 1^{n}\right)$. It is clear that the precise word problem for the pair $\left(W, 1^{n}\right)$ has a positive solution if and only if the bounded word problem has a negative solution for the pair $\left(W, 1^{n-1}\right)$ and has a positive solution for the pair $\left(W, 1^{n}\right)$. Since these facts can be verified in space $O\left(\max (\log |W|, \log n)(\log |W|)^{2}\right)$, we obtain a solution for the precise word problem in deterministic space $O\left(\max (\log |W|, \log n)(\log |W|)^{2}\right)$, as desired.

Now we describe an algorithm that solves the precise word problem for presentation (1.4) in polynomial time.

Recall that if $a_{i} \in \mathcal{A}$ and $U$ is a word over $\mathcal{A}^{ \pm 1}$, then $|U|_{a_{i}}$ denotes the number of all occurrences of letters $a_{i}, a_{i}^{-1}$ in $U$ and $|U|_{\bar{a}_{i}}:=|U|-|U|_{a_{i}}$.

Lemma 7.1. Let $\Delta$ be a reduced disk diagram over presentation (1.4), $W \equiv$ $\varphi\left(\left.\partial\right|_{0} \Delta\right)$ and $|W|_{\bar{a}_{1}}>0$. Then at least one of the following claims (a)-(b) holds true.

(a) There is a vertex $v$ of the path $P_{W}$ such that if $P_{W}($ fact, $v)=p_{1} p_{2}$ then $\left|\varphi\left(p_{1}\right)\right|_{\bar{a}_{1}}>0,\left|\varphi\left(p_{2}\right)\right|_{\bar{a}_{1}}>0$ and there is a special arc $s$ in $\Delta$ such that $s_{-}=\alpha(0)$ and $s_{+}=\alpha(v)$.

(b) Suppose $P_{W}\left(\right.$ fact, $\left.v_{1}, v_{2}\right)=p_{1} p_{2} p_{3}$ is a factorization of $P_{W}$ such that $\left|\varphi\left(p_{1}\right)\right|_{\bar{a}_{1}}=$ $0,\left|\varphi\left(p_{2}\right)\right|_{\bar{a}_{1}}=0$, and if $q_{i}=\alpha\left(p_{i}\right), i=1,2,3, e_{1}, e_{2}$ are the first, last, resp., edges of $q_{2}$, then neither of $e_{1}, e_{2}$ is an $a_{1}$-edge. Then there exists an $a_{k}$-band $\Gamma$ in $\Delta$ whose standard boundary is $\partial \Gamma=e_{i} r_{i} e_{3-i} r_{3-i}$, where $i=1$ if $\varphi\left(e_{1}\right) \in \mathcal{A}$ and $i=2$ if $\varphi\left(e_{1}\right)^{-1} \in \mathcal{A}$. In particular, the subdiagram $\Delta_{1}$ of $\Delta$ defined by $\partial \Delta_{1}=q_{1} r_{2}^{-1} q_{3}$, see Fig. 7.1, contains no faces and if $\Delta_{2}$ is the subdiagram of $\Delta$ defined by $\partial \Delta_{2}=q_{2} r_{1}^{-1}$, see Fig. 7.1, then $|\Delta(2)|=|\Gamma(2)|+\left|\Delta_{2}(2)\right|$ and $\varphi\left(q_{2}\right) \stackrel{\mathcal{G}_{3}}{=} a_{1}^{\ell}$, where $\ell=0$ if $k>2$, $\ell=n_{1} \ell_{0}$ for some integer $\ell_{0}$ if $\varphi\left(e_{1}\right)=a_{2}$, and $\ell=n_{2} \ell_{0}^{\prime}$ for some integer $\ell_{0}^{\prime}$ if $\varphi\left(e_{1}\right)=a_{2}^{-1}$. 


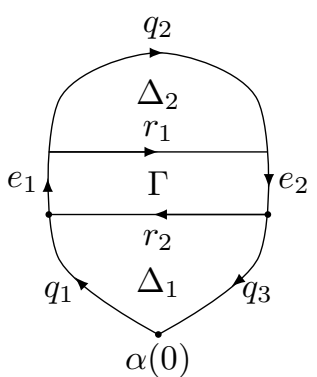

Fig. 7.1

Proof. Since $|W|_{\bar{a}_{1}}>0$, it follows that there is a unique factorization $P_{W}=p_{1} p_{2} p_{3}$ such that $\left|\varphi\left(p_{1}\right)\right|_{\bar{a}_{1}}=0,\left|\varphi\left(p_{2}\right)\right|_{\bar{a}_{1}}=0$, and the first and the last edges of $p_{2}$ are not $a_{1}$-edges. Denote $q_{i}=\alpha\left(p_{i}\right), i=1,2,3$, and let $e_{1}, f$ be the first, last, resp., edges of $q_{2}$.

Let $\varphi\left(e_{1}\right)=a_{k}^{\delta}, \delta= \pm 1$. Since $k>1$, there is an $a_{k}$-band $\Gamma$ whose standard boundary is $\partial \Gamma=e_{i} r_{i} e_{3-i} r_{3-i}$, where $i=1$ if $\varphi\left(e_{1}\right)=a_{k}$ and $i=2$ if $\varphi\left(e_{1}\right)=a_{k}^{-1}$.

First assume that $e_{2} \neq f$. By Lemma 6.1, $r_{2}$ is a special arc in $\Delta$. Since $\left|\varphi\left(q_{1}\right)\right|_{\bar{a}_{1}}=0$, there is a special arc $r$ in $\Delta$ such that $r_{-}=\alpha(0), r_{+}=\left(e_{1}\right)_{-}$. Applying Lemma 6.2 to special $\operatorname{arcs} r, r_{2}^{-1}$, we obtain a special arc $s$ such that $s_{-}=\alpha(0), s_{+}=\left(r_{2}\right)_{-}$. Now we can see from $e_{2} \neq f$ that claim (a) of Lemma 7.1 holds for $v=\left(p_{2}\right)_{+}$.

Suppose $e_{2}=f$. In view of the definition of an $a_{k}$-band and Lemma 6.1, we conclude that claim (b) of Lemma 7.1 holds true.

If $U \stackrel{\underline{G}_{3}}{=} a_{1}^{\ell}$ for some integer $\ell$, we let $\mu_{3}(U)$ denote the integer such that the precise word problem for presentation (1.4) holds for the pair $\left(U a_{1}^{-\ell}, \mu_{3}(U)\right)$. If $U \stackrel{\mathcal{S}_{3}}{\neq} a_{1}^{\ell}$ for any $\ell$, we set $\mu_{3}(U):=\infty$

Lemma 7.2. Let $U$ be a word such that $U \stackrel{\mathcal{G}_{3}}{=} a_{1}^{\ell}$ for some integer $\ell$ and $|U|_{\bar{a}_{1}}>0$. Then at least one of the following claims (a)-(b) holds true.

(a) There is a factorization $U \equiv U_{1} U_{2}$ such that $\left|U_{1}\right|_{\bar{a}_{1}},\left|U_{2}\right|_{\bar{a}_{1}}>0, U_{1} \stackrel{\mathcal{G}_{3}}{=} a_{1}^{\ell_{1}}$, $U_{2} \stackrel{\mathcal{G}_{3}}{=} a_{1}^{\ell_{2}}$ for some integers $\ell_{1}, \ell_{2}, \ell=\ell_{1}+\ell_{2}$, and $\mu_{3}(U)=\mu_{3}\left(U_{1}\right)+\mu_{3}\left(U_{2}\right)$.

(b) There is a factorization $U \equiv U_{1} a_{k}^{\delta} U_{2} a_{k}^{-\delta} U_{3}$, where $\left|U_{1}\right|_{\bar{a}_{1}}=\left|U_{2}\right|_{\bar{a}_{1}}=0$, $k \geq 2, \delta= \pm 1$, such that $U_{2} \stackrel{\mathcal{G}_{3}}{=} a_{1}^{\ell_{2}}$ for some integer $\ell_{2}$ and the following is true. If $k>2$, then $\ell_{2}=0$ and $U_{1} U_{3} \stackrel{0}{=} a_{1}^{\ell}$. If $k=2$ and $\delta=1$, then $\ell_{2} / n_{1}$ is an integer and $U_{1} a_{1}^{\ell_{2} n_{2} / n_{1}} U_{3} \stackrel{0}{=} a_{1}^{\ell}$. If $k=2$ and $\delta=-1$, then $\ell_{2} / n_{2}$ is an integer and $U_{1} a_{1}^{\ell_{2} n_{1} / n_{2}} U_{3} \stackrel{0}{=} a_{1}^{\ell}$.

Then, in case $k>2$, we have $\mu_{3}(U)=\mu_{3}\left(U_{2}\right)$; in case $k=2$ and $\delta=1$, we have $\mu_{3}(U)=\mu_{3}\left(U_{2}\right)+\left|\ell_{2} / n_{1}\right|$; in case $k=2$ and $\delta=-1$, we have $\mu_{3}(U)=$ $\mu_{3}\left(U_{2}\right)+\left|\ell_{2} / n_{2}\right|$.

Proof. Consider a disk diagram over (1.4) such that $\varphi\left(\left.\partial\right|_{0} \Delta\right) \equiv U a_{1}^{-\ell}$ and $|\Delta(2)|=$ $\mu_{3}(U)$. Clearly, $\Delta$ is reduced and Lemma 7.1 can be applied to $\Delta$.

Assume that Lemma 7.1(a) holds for $\Delta$. Then there is a special arc $s$ in $\Delta$ such that $s_{-}=\alpha(0)$ and $s_{+}=\left(q_{1}\right)_{+},\left.\partial\right|_{0} \Delta=q_{1} q_{2}$, and $\left|\varphi\left(q_{1}\right)\right|_{\bar{a}_{1}},\left|\varphi\left(q_{2}\right)\right|_{\bar{a}_{1}}>0$. Cutting 
$\Delta$ along the simple path $s$, we obtain two diagrams $\Delta_{1}, \Delta_{2}$ such that $\partial \Delta_{1}=q_{1} s^{-1}$, $\partial \Delta_{2}=q_{2} s$. Since $|\Delta(2)|=\mu_{3}(U)$ and $\varphi(s) \stackrel{0}{=} a_{1}^{\ell_{0}}$ for some $\ell_{0}$, it follows that if $U_{1}:=\varphi\left(q_{1}\right), U_{2} a_{1}^{-\ell}:=\varphi\left(q_{2}\right), U \equiv U_{1} U_{2}$, then $\left|\Delta_{i}(2)\right|=\mu_{3}\left(U_{i}\right), i=1,2$. Since $|\Delta(2)|=\left|\Delta_{1}(2)\right|+\left|\Delta_{2}(2)\right|$, we obtain

$$
\mu_{3}(U)=|\Delta(2)|=\left|\Delta_{1}(2)\right|+\left|\Delta_{2}(2)\right|=\mu_{3}\left(U_{1}\right)+\mu_{3}\left(U_{2}\right)
$$

as required.

Assuming that Lemma 7.1(b) holds for $\Delta$, we derive claim (b) from the definitions and Lemma 7.1(b).

Let $W$ be a word over $\mathcal{A}^{ \pm 1}$ and let $|W|_{\bar{a}_{1}}>0$. As in the proof of Theorem 1.2 let $W(i, j)$, where $1 \leq i \leq|W|$ and $0 \leq j \leq|W|$, denote the subword of $W$ that starts with the $i$ th letter of $W$ and has length $j$. If $W(i, j) \stackrel{\underline{G}_{3}}{=} a_{1}^{\ell_{i j}}$ for some integer $\ell_{i j}$, we set $\lambda(W(i, j)):=\ell_{i j}$. Otherwise, it is convenient to define $\lambda(W(i, j)):=\infty$.

We now compute the numbers $\lambda(W(i, j)), \mu_{3}(W(i, j))$ for all $i, j$ by the method of dynamic programming in which the parameter is $|W(i, j)|_{\bar{a}_{1}} \geq 0$. In other words, we compute the numbers $\lambda(W(i, j)), \mu_{3}(W(i, j))$ by induction on parameter $|W(i, j)|_{\bar{a}_{1}} \geq 0$.

To initialize, or to make the base step, we note that if $|W(i, j)|_{\bar{a}_{1}}=0$ then

$$
\lambda(W(i, j))=\ell_{i j}, \quad \mu_{3}(W(i, j))=0
$$

where $W(i, j) \stackrel{0}{=} a_{1}^{\ell_{i j}}$.

To make the induction step, assume that the numbers $\lambda\left(W\left(i^{\prime}, j^{\prime}\right)\right), \mu_{3}\left(W\left(i^{\prime}, j^{\prime}\right)\right)$ are already computed for all $W\left(i^{\prime}, j^{\prime}\right)$ such that $\left|W\left(i^{\prime}, j^{\prime}\right)\right|_{\bar{a}_{1}}<|W(i, j)|_{\bar{a}_{1}}$, where $W(i, j)$ is fixed and $|W(i, j)|_{\bar{a}_{1}}>0$.

Applying Lemma 7.2 to the word $U \equiv W(i, j)$, we consider all factorizations of the form

$$
U \equiv U_{1} U_{2}
$$

where $\left|U_{1}\right|_{\bar{a}_{1}},\left|U_{2}\right|_{\bar{a}_{1}}>0$. Since $U_{1}=W\left(i, j_{1}\right)$, where $j_{1}=\left|U_{1}\right|$ and $U_{2}=W(i+$ $\left.j_{1}, j-j_{1}\right)$, it follows from $\left|U_{1}\right|_{\bar{a}_{1}},\left|U_{2}\right|_{\bar{a}_{1}}<|U|_{\bar{a}_{1}}$ that the numbers $\lambda\left(U_{1}\right), \mu_{3}\left(U_{1}\right)$, $\lambda\left(U_{2}\right), \mu_{3}\left(U_{2}\right)$ are available by the induction hypothesis. Hence, we can compute the minimum

$$
M_{a}=\min \left(\mu_{3}\left(U_{1}\right)+\mu_{3}\left(U_{2}\right)\right)
$$

over all such factorizations $U \equiv U_{1} U_{2}$. If $M_{a}<\infty$ and $M_{a}=\mu_{3}\left(U_{1}^{\prime}\right)+\mu_{3}\left(U_{2}^{\prime}\right)$ for some factorization $U \equiv U_{1}^{\prime} U_{2}^{\prime}$, then we set $L_{a}:=\lambda\left(U_{1}^{\prime}\right)+\lambda\left(U_{2}^{\prime}\right)$.

We also consider a factorization for $U=W(i, j)$ of the form

$$
U \equiv U_{1} a_{k}^{\delta} U_{2} a_{k}^{-\delta} U_{3}
$$

where $\left|U_{1}\right|_{\bar{a}_{1}}=\left|U_{2}\right|_{\bar{a}_{1}}=0$, and $k \geq 2, \delta= \pm 1$.

If such a factorization (7.4) is impossible, we set $M_{b}=L_{b}=\infty$.

Assume that a factorization (7.4) exists (its uniqueness is obvious). Since

$$
U_{2}=W\left(i+\left|U_{1}\right|+1,\left|U_{2}\right|\right), \quad\left|U_{2}\right|_{\bar{a}_{1}}<|U|_{\bar{a}_{1}},
$$

it follows that the numbers $\lambda\left(U_{2}\right), \mu_{3}\left(U_{2}\right)$ are available. Denote $U_{1} U_{3} \stackrel{0}{=} a_{1}^{\ell}$.

If $k>2$ and $\lambda\left(U_{2}\right)=0$, we set

$$
L_{b}:=\ell, \quad M_{b}:=\mu_{3}\left(U_{2}\right) .
$$

If $k>2$ and $\lambda\left(U_{2}\right) \neq 0$, we set $L_{b}=M_{b}:=\infty$. 
If $k=2$ and $\delta=1$, we check whether $\lambda\left(U_{2}\right)$ is divisible by $n_{1}$. If so, we set

$$
L_{b}:=\ell+\lambda\left(U_{2}\right) n_{2} / n_{1}, \quad M_{b}:=\mu_{3}\left(U_{2}\right)+\left|\lambda\left(U_{2}\right) / n_{1}\right| .
$$

If $k=2, \delta=1$, and $\lambda\left(U_{2}\right)$ is not divisible by $n_{1}$, we set $L_{b}=M_{b}:=\infty$.

If $k=2$ and $\delta=-1$, we check whether $\lambda\left(U_{2}\right)$ is divisible by $n_{2}$. If so, we set

$$
L_{b}:=\ell+\lambda\left(U_{2}\right) n_{1} / n_{2}, \quad M_{b}:=\mu_{3}\left(U_{2}\right)+\left|\lambda\left(U_{2}\right) / n_{2}\right| .
$$

If $k=2, \delta=-1$, and $\lambda\left(U_{2}\right)$ is not divisible by $n_{2}$, we set $L_{b}=M_{b}:=\infty$.

It follows from Lemma 7.2 applied to the word $U=W(i, j)$ that if $U \stackrel{\mathcal{G}_{3}}{=} a_{1}^{\ell^{\prime}}$ for some integer $\ell^{\prime}$, then $\lambda(U)=\min \left(L_{a}, L_{b}\right)$ and $\mu_{3}(U)=\min \left(M_{a}, M_{b}\right)$. On the other hand, it is immediate that if $\lambda(U)=\infty$, then $L_{a}=L_{b}=\infty$ and $M_{a}=M_{b}=\infty$. Therefore, in all cases, we obtain that

$$
\lambda(U)=\min \left(L_{a}, L_{b}\right), \quad \mu_{3}(U)=\min \left(M_{a}, M_{b}\right) .
$$

This completes our computation of the numbers $\lambda(U), \mu_{3}(U)$ for $U=W(i, j)$.

It follows by the above inductive argument that, for every word $W(i, j)$ with finite $\lambda(W(i, j))$, we have

$$
\max \left(|\lambda(W(i, j))|, \mu_{3}(W(i, j))\right) \leq|W(i, j)| \max \left(\left|n_{1}\right|,\left|n_{2}\right|\right)^{|W(i, j)|_{\bar{a}_{1}} \leq 2^{O(|W|)} .}
$$

Hence, using binary representation for numbers $\lambda\left(W\left(i^{\prime}, j^{\prime}\right)\right), \mu_{3}\left(W\left(i^{\prime}, j^{\prime}\right)\right)$, we can run the foregoing computation of numbers $\lambda(U), \mu_{3}(U)$ for $U=W(i, j)$ in time $O\left(|W(i, j)|^{2}\right)$, including division of $\lambda\left(U_{2}\right)$ by $n_{1}$ and by $n_{2}$ to decide whether $\lambda\left(U_{2}\right) / n_{1}, \lambda\left(U_{2}\right) / n_{2}$ are integers and including additions to compute the minimum (7.3).

Since the number of subwords $W(i, j)$ of $W$ is bounded by $O\left(|W|^{2}\right)$, it follows that running time of the described algorithm that computes the numbers $\lambda(W), \mu_{3}(W)$ is $O\left(|W|^{4}\right)$. This implies that both the bounded word problem and the precise word problem for presentation (1.4) can be solved in deterministic time $O\left(|W|^{4}\right)$.

Theorem 1.4 is proved.

\section{Minimizing Diagrams over (1.2) And Proofs of Theorem 1.5 and Corollary 1.6}

Theorem 1.5. Both the diagram problem and the minimal diagram problem for group presentation (1.2) can be solved in deterministic space $O\left((\log |W|)^{3}\right)$ or in deterministic time $O\left(|W|^{4} \log |W|\right)$.

Furthermore, let $W$ be a word such that $W \stackrel{\mathcal{G}_{2}}{=} 1$ and let

$$
\tau(\Delta)=\left(\tau_{1}(\Delta), \ldots, \tau_{s_{\tau}}(\Delta)\right)
$$

be a tuple of integers, where the absolute value $\left|\tau_{i}(\Delta)\right|$ of each $\tau_{i}(\Delta)$ represents the number of certain vertices or faces in a disk diagram $\Delta$ over (1.2) such that $\varphi(\partial \Delta) \equiv W$. Then, in deterministic space $O\left((\log |W|)^{3}\right)$, one can algorithmically construct such a minimal diagram $\Delta$ which is also smallest relative to the tuple $\tau(\Delta)$ (the tuples are ordered lexicographically).

Proof. We start by proving the space part of Theorem 1.5.

Let $W$ be a nonempty word over $\mathcal{A}^{ \pm 1}$ and let $\Omega$ be a finite sequence of elementary operations that transforms the empty pseudobracket system for $W$ into a final pseudobracket system. By Lemma 4.7. $W \stackrel{\mathcal{G}_{2}}{=} 1$ and there is a diagram $\Delta$ over (1.2) such that $\varphi\left(\left.\partial\right|_{0} \Delta\right) \equiv W$. We now describe an explicit construction of such 
a diagram $\Delta=\Delta(\Omega)$ with property (A) based on the sequence $\Omega$. Note that this construction is based on the proof of Lemma 4.7 and essentially follows that proof. The question on how to construct the sequence $\Omega$ will be addressed later.

Let $B_{0}, B_{1}, \ldots, B_{\ell}$ be the sequence of pseudobracket systems associated with $\Omega$, where $B_{0}$ is empty and $B_{\ell}$ is final. We will construct $\Delta(\Omega)$ by induction on $i$. Let $B_{i}$ be obtained from $B_{i-1}, i \geq 1$, by an elementary operation $\sigma$ and let $b^{\prime} \in B_{i}$ be the pseudobracket obtained from $b, c \in B_{i-1}$ by $\sigma$. Here we assume that one of $b, c$ (or both) could be missing.

If $\sigma$ is an addition, then we do not do anything.

Assume that $\sigma$ is an extension of type 1 or type 2 on the left and $a\left(b^{\prime}\right)=e_{1} a(b)$, where $e_{1}$ is an edge of $P_{W}$ and $\varphi\left(e_{1}\right)=a_{b(3)}^{\varepsilon_{1}}$. Here we use the notation of the definition of an extension of type 1 or type 2 on the left. Then we construct $\Delta(\Omega)$ so that the edge $\alpha\left(e_{1}\right)=e$ belongs to the boundary $\partial \Delta(\Omega)$ of $\Delta(\Omega)$ and $e^{-1}$ belongs

to $\partial \Pi$, where $\Pi$ is a face of $\Delta(\Omega)$ such that $\varphi(\partial \Pi) \equiv a_{b(3)}^{-\varepsilon_{1} b(4)}$. Note that the label $\varphi(\partial \Pi)$ is uniquely determined by either of the pseudobrackets $b, b^{\prime}$.

The case of an extension of type 1 or type 2 on the right is analogous.

Suppose that $\sigma$ is an extension of type 3 and $a\left(b^{\prime}\right)=e_{1} a(b) e_{2}$, where $e_{1}, e_{2}$ are edges of $P_{W}$ with $\varphi\left(e_{1}\right)=\varphi\left(e_{2}\right)^{-1}$. Here we use the notation of the definition of an extension of type 3 . Then we construct $\Delta(\Omega)$ so that $\alpha\left(e_{1}\right)=\alpha\left(e_{2}\right)^{-1}$ and both $\alpha\left(e_{1}\right), \alpha\left(e_{2}\right)$ belong to the boundary $\partial \Delta(\Omega)$ of $\Delta(\Omega)$.

If $\sigma$ is a turn or a merger, then we do not do anything to the diagram $\Delta(\Omega)$ under construction.

Performing these steps during the process of elementary operations $\Omega$, we will construct, i.e., effectively describe, a required diagram $\Delta(\Omega)$ such that $|\Delta(\Omega)(2)|=$ $b_{F}(6)$, where $b_{F}$ is the final pseudobracket of $B_{\ell}$, and $\varphi\left(\left.\partial\right|_{0} \Delta(\Omega)\right) \equiv W$. Note that this particular diagram $\Delta(\Omega)$, depending on the sequence $\Omega$, may not be minimal or even reduced, however, as in the proof of Lemma 4.7 our construction guarantees that $\Delta(\Omega)$ does have the property (A), i.e., the property that if $e$ is an edge of the boundary path $\partial \Pi$ of a face $\Pi$ of $\Delta(\Omega)$, then $e^{-1} \in \partial \Delta(\Omega)$.

We also observe that, in the proof of Lemma 4.6, for a given pair $\left(W, \Delta_{W}\right)$, where $\Delta_{W}$ is a diagram over (1.2) such that $\varphi\left(\left.\partial\right|_{0} \Delta_{W}\right) \equiv W$, we constructed by induction an operational sequence $\Omega=\Omega\left(\Delta_{W}\right)$ of elementary operations that convert the empty bracket system into the final bracket system and has size bounded by

$$
(11|W|, C(\log |W|+1))) \text {. }
$$

Recall that every bracket system is also a pseudobracket system. Looking at details of the construction of the sequence $\Omega\left(\Delta_{W}\right)$, we can see that if we reconstruct a diagram $\Delta\left(\Omega\left(\Delta_{W}\right)\right)$, by utilizing our above algorithm based upon the sequence $\Omega\left(\Delta_{W}\right)$, then the resulting diagram will be identical to the original diagram $\Delta_{W}$, hence, in this notation, we can write

$$
\Delta\left(\Omega\left(\Delta_{W}\right)\right)=\Delta_{W} .
$$

Recall that Savitch's conversion [38, see also [3], 32, of nondeterministic computations in space $S$ and time $T$ into deterministic computations in space $O(S \log T)$ simulates possible nondeterministic computations by using tuples of configurations, also called instantaneous descriptions, of cardinality $O(\log T)$. Since a configuration takes space $S$ and the number of configurations that are kept in memory at every moment is at most $O(\log T)$, the space bound $O(S \log T)$ becomes evident. 
Furthermore, utilizing the same space $O(S \log T)$, one can use Savitch's algorithm to compute an actual sequence of configurations that transform an initial configuration into a final configuration. To do this, we consider every configuration $\psi$ together with a number $k, 0 \leq k \leq T$, thus replacing every configuration $\psi$ by a pair $(\psi, k)$, where $k$ plays the role of counter. We apply Savitch's algorithm to such pairs $(\psi, k)$, so that $\left(\psi_{2}, k+1\right)$ is obtained from $\left(\psi_{1}, k\right)$ by a single operation whenever $\psi_{2}$ is obtained from $\psi_{1}$ by a single command. If $\psi$ is a final configuration, then we also assume that $(\psi, k+1)$ can be obtained from $(\psi, k), 0 \leq k \leq T-1$, by a single operation.

Now we apply Savitch's algorithm to two pairs $\left(\psi_{0}, 0\right),\left(\psi_{F}, T\right)$, where $\psi_{0}$ is an initial configuration and $\psi_{F}$ is a final configuration. If Savitch's algorithm accepts these two pairs $\left(\psi_{0}, 0\right),\left(\psi_{F}, T\right)$, i.e., the algorithm turns the pair $\left(\psi_{0}, 0\right)$ into $\left(\psi_{F}, T\right)$, then there will be a corresponding sequence

$$
\left(\psi_{0}, 0\right),\left(\psi_{1}, 1\right), \ldots,\left(\psi_{i}, i\right), \ldots,\left(\psi_{F}, T\right)
$$

of pairs such that $\left(\psi_{i}, i\right)$ is obtained from $\left(\psi_{i-1}, i-1\right), i \geq 1$, by a single operation. Observe that we can retrieve any term, say, the $k$ th term of the sequence (8.3), by rerunning Savitch's algorithm and memorizing a current pair $(\psi, k)$ in a separate place in memory. Note that the current value of $(\psi, k)$ may change many times during computations but in the end it will be the desired pair $\left(\psi_{k}, k\right)$. Clearly, the space needed to run this modified Savitch's algorithm is still $O(S \log T)$ and, consecutively outputting these pairs $\left(\psi_{0}, 0\right),\left(\psi_{1}, 1\right), \ldots$, we will construct the required sequence (8.3) of configurations.

Coming back to our specific situation, we recall that a pseudobracket system plays the role of a configuration, the empty pseudobracket system is an initial configuration and a final pseudobracket system is a final configuration. Therefore, it follows from Lemmas 4.6 4.7, and the foregoing equality (8.2) that picking the empty pseudobracket system $B_{0}$ and a final pseudobracket system $\left\{b_{F}\right\}$, where $b_{F}=\left(0,|W|, 0,0,0, n^{\prime}\right)$, we can use Savitch's algorithm to verify in deterministic space $O\left((\log |W|)^{3}\right)$ whether $W \stackrel{\mathcal{G}_{2}}{=} 1$ and whether there is a disk diagram $\Delta$ over (1.2) such that $\varphi(\partial \Delta) \equiv W$ and $|\Delta(2)|=n^{\prime}$. In addition, if the algorithm accepts, then the algorithm also constructs an operational sequence $B_{0}, B_{1}, \ldots, B_{\ell}=\left\{b_{F}\right\}$ of pseudobracket systems of size bounded by (8.1) which, as was discussed above, can be used to construct a disk diagram $\Delta_{1}$ with property (A) such that $\varphi\left(\partial \Delta_{1}\right) \equiv W$ and $\left|\Delta_{1}(2)\right|=n^{\prime}$. It is clear that the entire construction of $\Delta_{1}$ can be done in deterministic space $O\left((\log |W|)^{3}\right)$, as desired.

To construct a minimal disk diagram for $W$, we consecutively choose $n^{\prime}=$ $0,1,2, \ldots$ and run Savitch's algorithm to determine whether the algorithm can turn the empty pseudobracket system into a final pseudobracket system $\left\{b_{F}\right\}$, where $b_{F}(6)=n^{\prime}$. If $n_{0}$ is the minimal such $n^{\prime}$, then we run Savitch's algorithm again to construct an operational sequence of pseudobracket systems and a corresponding disk diagram $\Delta_{0}$ such that $\varphi\left(\partial \Delta_{0}\right) \equiv W$ and $\left|\Delta_{0}(2)\right|=n_{0}$. Similarly to the above arguments, it follows from Lemmas 4.6 4.7, and from the equality $\Delta\left(\Omega\left(\Delta_{W}\right)\right)=\Delta_{W}$, see (8.2), that $\Delta_{0}$ is a minimal diagram and the algorithm uses space $O\left((\log |W|)^{3}\right)$. Thus, the minimal diagram problem can be solved for presentation (1.2) in deterministic space $O\left((\log |W|)^{3}\right)$. 
Now we will discuss the additional statement of Theorem 1.5. For a diagram $\Delta$ over presentation (1.2) such that $\varphi\left(\left.\partial\right|_{0} \Delta\right) \equiv W$ and $\Delta$ has property (A), we consider a parameter

$$
\tau(\Delta)=\left(\tau_{1}(\Delta), \ldots, \tau_{s_{\tau}}(\Delta)\right)
$$

where $\tau_{i}(\Delta)$ are integers that satisfy $0 \leq\left|\tau_{i}(\Delta)\right| \leq C_{\tau}|W|, C_{\tau}>0$ is a fixed constant, and that represent numbers of certain vertices, edges, faces of $\Delta$ (we may also consider some functions of the numbers of certain vertices, edges, faces of $\Delta$ that are computable in space $O\left((\log |W|)^{3}\right)$ and whose absolute values do not exceed $\left.C_{\tau}|W|\right)$.

For example, if we set $\tau_{1}(\Delta):=|\Delta(2)|$ then we would be constructing a minimal diagram which is also smallest with respect to $\tau_{2}(\Delta)$, then smallest with respect to $\tau_{3}(\Delta)$ and so on. Let us set $\tau_{2}(\Delta):=\left|\Delta(2)_{a_{1}^{ \pm n_{1}}}\right|$, where $\left|\Delta(2)_{a_{1}^{ \pm n_{1}}}\right|$ is the number of faces $\Pi_{2}$ in $\Delta$ such that $\varphi\left(\partial \Pi_{2}\right) \equiv a_{1}^{ \pm n_{1}}, \tau_{3}(\Delta):=\left|\Delta(2)_{a_{2}^{n_{2}}}\right|$, where $\left|\Delta(2)_{a_{2}}\right|$ is the number of faces $\Pi_{3}$ in $\Delta$ such that $\varphi\left(\partial \Pi_{3}\right) \equiv a_{2}^{n_{2}}$.

We may also consider functions such as $\tau_{4}(\Delta):=\sum_{k_{4,1} \leq|\partial \Pi| \leq k_{4,2}}|\partial \Pi|$, where the summation takes place over all faces $\Pi$ in $\Delta$ such that $k_{4,1} \leq|\partial \Pi| \leq k_{4,2}$, where $k_{4,1}, k_{4,2}$ are fixed integers with $0 \leq k_{4,1} \leq k_{4,2} \leq|W|$. Note that the latter bound follows from Lemma 2.2 (a).

If $v$ is a vertex of $\Delta$, let $\operatorname{deg} v$ denote the degree of $v$, i.e., the number of oriented edges $e$ of $\Delta$ such that $e_{+}=v$. Also, if $v$ is a vertex of $\Delta$, let $\operatorname{deg}^{F} v$ denote the face degree of $v$, i.e., the number of faces $\Pi$ in $\Delta$ such that $v \in \partial \Pi$. Note that it follows from Lemma 2.2 (a) that every face $\Pi$ of a disk diagram $\Delta$ with property (A) can contribute at most 1 to this sum.

We may also consider entries in $\tau(\Delta)$ that count the number of vertices in $\Delta$ of certain degree. Note that doing this would be especially interesting and meaningful when the presentation (1.2) contains no defining relations, hence, diagrams over (1.2) are diagrams over the free group $F(\mathcal{A})=\langle\mathcal{A} \| \varnothing\rangle$ with no relations. For instance, we may set $\tau_{5}(\Delta):=\left|\Delta(0)_{\geq 3}\right|$, where $\left|\Delta(0)_{\geq 3}\right|$ is the number of vertices in $\Delta$ of degree at least 3 , and set $\tau_{6}(\Delta):=\left|\Delta(0)_{\left[k_{6,1}, k_{6,2}\right]}^{F}\right|$, where $\left|\Delta(0)_{\left[k_{6,1}, k_{6,2}\right]}^{F}\right|$ is the number of vertices $v$ in $\Delta$ such that $k_{6,1} \leq \operatorname{deg}^{F} v \leq k_{6,2}$, where $k_{6,1}, k_{6,2}$ are fixed integers with $0 \leq k_{6,1} \leq k_{6,2} \leq|W|$.

Our goal is to make some modification of the foregoing algorithm that would enable us to construct a disk diagram $\Delta$ over (1.2) such that $\varphi\left(\left.\partial\right|_{0} \Delta_{W}\right) \equiv W$, $\Delta$ has property (A), and $\Delta$ is smallest relative to the parameter $\tau(\Delta)$. Recall that the tuples $\tau(\Delta)$ are ordered in the standard lexicographical way. Note that if $\tau_{1}(\Delta)=-|\Delta(2)|$ then $\Delta$ would be a diagram for $W$ with property (A) which has the maximal number of faces.

Our basic strategy remains unchanged, although we will need more complicated bookkeeping (to be introduced below). We start by picking a value for the parameter $\widetilde{\tau}=\left(\widetilde{\tau}_{1}, \ldots, \widetilde{\tau}_{s_{\tau}}\right)$, where $\widetilde{\tau}_{i}$ are fixed integers with $\left|\widetilde{\tau}_{i}\right| \leq C_{\tau}|W|$. As before, using Lemmas 4.6, 4.7 and the identity $\Delta\left(\Omega\left(\Delta_{W}\right)\right)=\Delta_{W}$, see (8.2), we can utilize Savitch's algorithm which verifies, in deterministic space $O\left((\log |W|)^{3}\right)$, whether the empty pseudobracket system $B_{0}$ can be turned by elementary operations into a final pseudobracket system $B=\left\{b_{F}\right\}$ which also changes the $\tau$-parameter from $(0,0, \ldots, 0)$ to $\widetilde{\tau}$. If this is possible, then the algorithm also computes an operational sequence $B_{0}, B_{1}, \ldots, B_{\ell}=B$ of pseudobracket systems and, based on this sequence, constructs a disk diagram $\Delta$ such that $\varphi(\partial \Delta) \equiv W, \Delta$ has property (A) 
and $\tau(\Delta)=\widetilde{\tau}$. To make sure that $\Delta$ is smallest relative to the parameter $\tau(\Delta)$, we can use Savitch's algorithm for every $\widetilde{\tau}^{\prime}<\widetilde{\tau}$ to verify that the empty pseudobracket system $B_{0}$ may not be turned by elementary operations into a final pseudobracket system $B=\left\{b_{F}\right\}$ so that the $\tau$-parameter concurrently changes from $(0,0, \ldots, 0)$ to $\widetilde{\tau}^{\prime}$. This concludes the description of our algorithm for construction of a diagram $\Delta$ over (1.2) such that $\varphi\left(\left.\partial\right|_{0} \Delta_{W}\right) \equiv W, \Delta$ has property (A) and $\Delta$ is smallest relative to the parameter $\tau(\Delta)$.

Let us describe necessary modifications in bookkeeping. First, we add six more integer entries to each pseudobracket $b$. Hence, we now have

$$
b=(b(1), \ldots, b(6), b(7), \ldots, b(12)),
$$

where $b(1), \ldots, b(6)$ are defined exactly as before, $b(7), \ldots, b(12)$ are integers such that

$$
\begin{array}{ll}
b(7)+b(8)=b(5), & 0 \leq b(7), b(8) \leq b(5) \quad \text { if } \quad b(5) \geq 0, \\
b(7)+b(8)=b(5), & b(5) \leq b(7), b(8) \leq 0 \quad \text { if } \quad b(5) \leq 0 .
\end{array}
$$

The numbers $b(9), \ldots, b(12)$ satisfy the inequalities

$$
0 \leq b(9), \ldots, b(12) \leq|W|
$$

and the equalities $b(7)=b(8)=b(11)=b(12)=0$ whenever $b$ has type PB1. The purpose of these new entries, to be specified below, is to keep track of the information on degrees and face degrees of the vertices of the diagram $\Delta(\Omega)$ over (1.2) being constructed by means of an operational sequence $\Omega$ of elementary operations.

We now inductively describe the changes over the entries $b(7), \ldots, b(12)$ and over the parameter $\tau(\Delta)=\left(\tau_{1}(\Delta), \ldots, \tau_{s_{\tau}}(\Delta)\right)$ that are done in the process of performance of an operational sequence $\Omega$ of elementary operations over pseudobracket systems that change the empty pseudobracket system into a final pseudobracket system for $W$, where $W \stackrel{\underline{G}_{2}}{=} 1$ and $|W|>0$.

To make the inductive definitions below easier to understand, we will first make informal remarks about meaning of the new entries $b(7), \ldots, b(12)$.

Suppose that $b$ is a pseudobracket of type PB1. Then $b(7)=b(8)=0$ and $b(11)=b(12)=0$. The entry $b(9)$ represents the current (or intermediate) degree $\operatorname{deg} v$ of the vertex $v=\alpha(b(1))=\alpha(b(2))$ which is subject to change in process of construction of the diagram $\Delta(\Omega)$. The entry $b(10)$ represents the current face degree $\operatorname{deg}^{F} v$ of the vertex $v=\alpha(b(1))=\alpha(b(2))$ which is also subject to change. For example, if a pseudobracket $b^{\prime}$ is obtained from pseudobrackets $b, c$ of type PB1 by a merger, then $b^{\prime}(9):=b(9)+c(9)$ and $b^{\prime}(10):=b(10)+c(10)$. Note that $b^{\prime}(9), b^{\prime}(10)$ are still intermediate degrees of the vertex $\alpha\left(b^{\prime}(1)\right)=\alpha\left(b^{\prime}(2)\right)$, i.e., $b^{\prime}(9), b^{\prime}(10)$ are not actual degrees of $\alpha\left(b^{\prime}(1)\right)$, because there could be more elementary operations such as extension of type 3 , mergers, and turns that could further increase $b^{\prime}(9), b^{\prime}(10)$.

Assume that $b$ is a pseudobracket of type PB2. Then the entry $b(7)$ represents the exponent in the power $a_{b(3)}^{b(7)} \equiv \varphi\left(u_{1}^{-1}\right)$, where $u_{1}$ is the arc of $\partial \Pi$, where $\Pi$ is a face such that $\left(u_{1}\right)_{-}=\alpha(v),\left(u_{1}\right)_{+}=\alpha(b(1))$, see Fig. 8.1, and $v$ is the vertex of $P_{W}$ at which a turn operation was performed to get a pseudobracket $\bar{b}$ of type PB2 
which, after a number of extensions of type 1 and mergers with pseudobrackets of type PB1, becomes $b$.

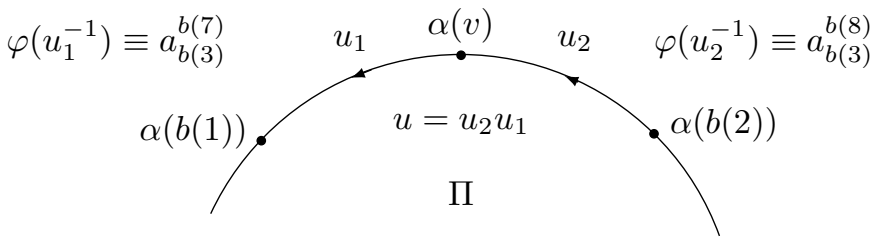

Fig. 8.1

Similarly, the entry $b(8)$ represents the exponent in the power $a_{b(3)}^{b(8)} \equiv \varphi\left(u_{2}^{-1}\right)$, where $u_{2}$ is the arc of $\partial \Pi$ such that $\left(u_{2}\right)_{-}=\alpha(b(2)),\left(u_{1}\right)_{+}=\alpha(v)$, and $v$ is the vertex of $P_{W}$ defined as above, see Fig. 8.1. Since $\varphi\left(u^{-1}\right) \equiv a_{b(3)}^{b(5)}$, where $u=u_{2} u_{1}$ is the arc of $\partial \Pi$ defined by $u_{-}=\alpha(b(2)), u_{+}=\alpha(b(1))$, see Fig. 8.1, it follows from the definitions that the conditions (8.5) - (8.6) hold true.

As in the above case when $b$ has type PB2, the entry $b(9)$ represents the current (or intermediate) degree $\operatorname{deg} v_{1}$ of the vertex $v_{1}=\alpha(b(1))$ which is subject to change in the process of construction of $\Delta(\Omega)$. As above, the entry $b(10)$ represents the current face degree $\operatorname{deg}^{F} v_{1}$ of $v_{1}=\alpha(b(1))$ which is also subject to change. The entry $b(11)$ is the intermediate degree $\operatorname{deg} v_{2}$ of the vertex $v_{2}=\alpha(b(2))$ and $b(12)$ represents the current face degree $\operatorname{deg}^{F} v_{2}$ of the vertex $v_{2}=\alpha(b(2))$.

Our description for meaning of the new entries $b(7), \ldots, b(12)$ in thus augmented pseudobracket $b$ is complete and we now inductively describe the changes over these new entries $b(7), \ldots, b(12)$ and over the parameter $\tau(\Delta)$ in process of performance of a sequence $\Omega$ of elementary operations over pseudobracket systems that change the empty pseudobracket system into a final pseudobracket system for a word $W$ such that $W \stackrel{\mathcal{G}_{2}}{=} 1$ and $|W|>0$.

As above, assume that $\Omega$ is a sequence of elementary operations that change the empty pseudobracket system into a final pseudobracket system for $W$. Let $B_{0}, B_{1}, \ldots, B_{\ell}$ be the corresponding to $\Omega$ sequence of pseudobracket systems, where $B_{0}$ is empty and $B_{\ell}$ is final.

If $b \in B_{i+1}, i \geq 0$, is a starting pseudobracket, then we set

$$
b(7)=b(8)=b(9)=b(10)=b(11)=b(12)=0 .
$$

As was mentioned above, the entries $b(1), \ldots, b(6)$ in every pseudobracket $b \in$ $\cup_{j=0}^{\ell} B_{j}$ are defined exactly as before.

Suppose that a pseudobracket $b^{\prime} \in B_{i+1}, i \geq 1$, is obtained from $b \in B_{i}$ by an extension of type 1 on the left and $a\left(b^{\prime}\right)=e_{1} a(b)$, where $a\left(b^{\prime}\right), a(b)$ are the arcs of $b^{\prime}, b$, resp., and $e_{1}$ is an edge of $P_{W}, \varphi\left(e_{1}\right)=a_{b(3)}^{\varepsilon_{1}}, \varepsilon_{1}= \pm 1$. Recall that both pseudobrackets $b^{\prime}, b$ have type PB2.

First we assume that $b(5) \neq 0$, i.e., one of $b(7), b(8)$ is different from 0 . Then we set

$$
\begin{aligned}
& b^{\prime}(7):=b(7)+\varepsilon_{1}, \quad b^{\prime}(8):=b(8), \quad b^{\prime}(9):=2, \\
& b^{\prime}(10):=1, \quad b^{\prime}(11):=b(11), \quad b^{\prime}(12):=b(12) .
\end{aligned}
$$

Note that it follows from the definitions that $\left|b^{\prime}(7)\right|=|b(7)|+1$. We also update those entries in the sequence $\tau(\Delta)$ that are affected by the fact that $v_{1}=\alpha(b(1))$ is 
now known to be a vertex of the diagram $\Delta(\Omega)$ (which is still under construction) such that $\operatorname{deg} v_{1}=b(9)$ and $\operatorname{deg}^{F} v_{1}=b(10)$.

For example, if $\tau_{5}(\Delta)=\left|\Delta(0)_{\geq 3}\right|$, where $\left|\Delta(0)_{\geq 3}\right|$ is the number of vertices in $\Delta$ of degree at least 3 , and $b(9) \geq 3$, then $\tau_{5}(\Delta)$ is increased by 1 . If $\tau_{6}(\Delta)=$ $\left|\Delta(0)_{\left[k_{6,1}, k_{6,2}\right]}^{F}\right|$, where $\left|\Delta(0)_{\left[k_{6,1}, k_{6,2}\right]}^{F}\right|$ is the number of vertices $u$ in $\Delta$ such that $k_{6,1} \leq \operatorname{deg}^{F} u \leq k_{6,2}$, where $k_{6,1}, k_{6,2}$ are fixed integers with $0 \leq k_{6,1} \leq k_{6,2} \leq|W|$, and if $\operatorname{deg}^{F} \alpha(b(1))=b(10)$ satisfies $k_{6,1} \leq b(10) \leq k_{6,2}$, then we increase $\tau_{6}(\Delta)$ by 1.

Suppose that $b(5)=0$, i.e., $b(7)=b(8)=0$. Then we set

$$
\begin{aligned}
& b^{\prime}(7):=\varepsilon_{1}, \quad b^{\prime}(8):=0, \quad b^{\prime}(9):=2, \\
& b^{\prime}(10):=1, \quad b^{\prime}(11):=b(9), \quad b^{\prime}(12):=b(10) .
\end{aligned}
$$

These formulas, see also (8.7)-(8.8) and other formulas below, reflect the convention that, for a pseudobracket $b$ of type PB2, the information about edge and face degrees of the vertex $\alpha(b(2))$ is stored in components $b(9), b(10)$, resp., whenever $\alpha(b(1))=\alpha(b(2))$, i.e., $b(5)=0$. However, when $\alpha(b(1)) \neq \alpha(b(2))$, i.e., $b(5) \neq 0$, this information for the vertex $\alpha(b(2))$ is separately kept in components $b(11), b(12)$, whereas this information for the vertex $\alpha(b(1))$ is stored in components $b(9), b(10)$. In the current case $b(5)=0$, no change to $\tau(\Delta)$ is done.

The case of an extension of type 1 on the left is complete.

Assume that a pseudobracket $b^{\prime} \in B_{i+1}, i \geq 1$, is obtained from $b \in B_{i}$ by an extension of type 1 on the right. This case is similar to its "left" analogue studied above but not quite symmetric, because of the way we keep information about degrees of vertices and we will write down all necessary formulas. As above, both pseudobrackets $b^{\prime}, b$ have type PB2.

Let $a\left(b^{\prime}\right)=a(b) e_{2}$, where $a\left(b^{\prime}\right), a(b)$ are the arcs of $b^{\prime}, b$, resp., and $e_{2}$ is an edge of $P_{W}, \varphi\left(e_{2}\right)=a_{b(3)}^{\varepsilon_{2}}, \varepsilon_{2}= \pm 1$. If $b(5) \neq 0$, then we define

$$
\begin{aligned}
& b^{\prime}(7):=b(7), \quad b^{\prime}(8):=b(8)+\varepsilon_{2}, \quad b^{\prime}(9):=b(9), \\
& b^{\prime}(10):=b(10), \quad b^{\prime}(11):=2, \quad b^{\prime}(12):=1 .
\end{aligned}
$$

Note that it follows from the definitions that $\left|b^{\prime}(8)\right|=|b(8)|+1$. We also update those entries in the sequence $\tau(\Delta)$ that are affected by the fact that $v_{2}=\alpha(b(2))$ is a vertex of $\Delta(\Omega)$ (which is still under construction) such that $\operatorname{deg} v_{2}=b(11)$ and $\operatorname{deg}^{F} v_{2}=b(12)$.

For example, if $\tau_{5}(\Delta)=\left|\Delta(0)_{\geq 3}\right|$ and $b(11) \geq 3$, then $\tau_{5}(\Delta)$ is increased by 1. If $\tau_{6}(\Delta)=\left|\Delta(0)_{\left[k_{6,1}, k_{6,2}\right]}^{F}\right|$ and if $\operatorname{deg}^{F} \alpha(b(2))=b(12)$ satisfies the inequalities $k_{6,1} \leq b(12) \leq k_{6,2}$, then we increase $\tau_{6}(\Delta)$ by 1 .

If $b(5)=0$, then, to define $b^{\prime}$, we again use formulas (8.7) - (8.8) but we do not make any changes to $\tau(\Delta)$.

The case of an extension of type 1 on the right is complete.

Suppose that a pseudobracket $b^{\prime} \in B_{i+1}, i \geq 1$, is obtained from $b \in B_{i}$ by an extension of type 2 on the left. Denote $a\left(b^{\prime}\right)=e_{1} a(b)$, where $a\left(b^{\prime}\right), a(b)$ are the arcs of $b^{\prime}, b$, resp., and $e_{1}$ is an edge of $P_{W}, \varphi\left(e_{1}\right)=a_{b(3)}^{\varepsilon_{1}}, \varepsilon_{1}= \pm 1$. Recall that $b$ has type PB2 and $b^{\prime}$ has type PB1. 
First we assume that $b(5) \neq 0$, i.e., one of $b(7), b(8)$ is different from 0 . Then we set

$$
\begin{aligned}
& b^{\prime}(7):=0, \quad b^{\prime}(8):=0, \quad b^{\prime}(9):=b(11), \\
& b^{\prime}(10):=b(12), \quad b^{\prime}(11):=0, \quad b^{\prime}(12):=0 .
\end{aligned}
$$

We also update the sequence $\tau(\Delta)$ according to the information that $v_{1}=\alpha(b(1))$ is a vertex of $\Delta(\Omega)$ (which is still under construction) such that $\operatorname{deg} v_{1}=b(9)$, $\operatorname{deg}^{F} v_{1}=b(10)$, and that $\Delta(\Omega)$ contains a face $\Pi_{b}$ such that $\varphi\left(\partial \Pi_{b}\right) \equiv a_{b(3)}^{-\varepsilon_{1} b(4)}$.

For example, if $\tau_{5}(\Delta)=\left|\Delta(0)_{\geq 3}\right|$ and $b(9) \geq 3$, then $\tau_{5}(\Delta)$ is increased by 1 . If $\tau_{6}(\Delta)=\left|\Delta(0)_{\left[k_{6,1}, k_{6,2}\right]}^{F}\right|$ and if $\operatorname{deg}^{F} \alpha(b(1))=b(10)$ satisfies $k_{6,1} \leq b(10) \leq k_{6,2}$, then we increase $\tau_{6}(\Delta)$ by 1 . If $\tau_{2}(\Delta)=\left|\Delta(2)_{a_{1}^{ \pm n_{1}}}\right|$, where $\left|\Delta(2)_{a_{1}^{ \pm n_{1}}}\right|$ is the number of faces $\Pi_{2}$ in $\Delta$ such that $\varphi\left(\partial \Pi_{2}\right) \equiv a_{1}^{ \pm n_{1}}$, and $b(3)=1, b(4)=n_{1}$, then we increase $\tau_{2}(\Delta)$ by 1 . If $\tau_{3}(\Delta)=\left|\Delta(2) a_{2}^{n_{2}}\right|$, where $\left|\Delta(2) a_{2}^{n_{2}}\right|$ is the number of faces $\Pi_{3}$ in $\Delta$ such that $\varphi\left(\partial \Pi_{3}\right) \equiv a_{2}^{n_{2}}$, and $b(3)=2,-\varepsilon_{1} b(4)=n_{2}$, then we increase $\tau_{3}(\Delta)$ by 1. If $\tau_{4}(\Delta)=\sum_{k_{4,1} \leq|\partial \Pi| \leq k_{4,2}}|\partial \Pi|$, where the summation takes place over all faces $\Pi$ in $\Delta$ such that $k_{4,1} \leq|\partial \Pi| \leq k_{4,2}$, where $k_{4,1}, k_{4,2}$ are fixed integers with $0 \leq k_{4,1} \leq k_{4,2} \leq|W|$, and $k_{4,1} \leq b(4) \leq k_{4,2}$, then we increase $\tau_{4}(\Delta)$ by 1 .

Now assume that $b(5)=0$, i.e., $b(7)=b(8)=0$, and so $b(4)=1$. Then we set

$$
\begin{aligned}
& b^{\prime}(7):=0, \quad b^{\prime}(8):=0, \quad b^{\prime}(9):=b(9), \\
& b^{\prime}(10):=b(10), \quad b^{\prime}(11):=0, \quad b^{\prime}(12):=0 .
\end{aligned}
$$

We also update the tuple $\tau(\Delta)$ according to the information that $\Delta(\Omega)$ contains a face $\Pi_{b}$ such that $\varphi\left(\partial \Pi_{b}\right) \equiv a_{b(3)}^{-\varepsilon_{1}}$.

The case of an extension of type 2 on the left is complete.

Suppose that a pseudobracket $b^{\prime} \in B_{i+1}, i \geq 1$, is obtained from $b \in B_{i}$ by an extension of type 2 on the right. This case is similar to its "left" analogue discussed above but is not quite symmetric and we will write down all necessary formulas.

Denote $a\left(b^{\prime}\right)=a(b) e_{2}$, where $a\left(b^{\prime}\right), a(b)$ are the arcs of $b^{\prime}, b$, resp., and $e_{2}$ is an edge of $P_{W}, \varphi\left(e_{2}\right)=a_{b(3)}^{\varepsilon_{2}}, \varepsilon_{2}= \pm 1$. Recall that $b$ has type PB2 and $b^{\prime}$ has type PB1.

First we assume that $b(5) \neq 0$. Then we set

$$
\begin{aligned}
& b^{\prime}(7):=0, \quad b^{\prime}(8):=0, \quad b^{\prime}(9):=b(9), \\
& b^{\prime}(10):=b(10), \quad b^{\prime}(11):=0, \quad b^{\prime}(12):=0 .
\end{aligned}
$$

We also update the sequence $\tau(\Delta)$ according to the information that $v_{2}=\alpha(b(2))$ is a vertex of $\Delta(\Omega)$ (which is still under construction) such that $\operatorname{deg} v_{2}=b(11)$, $\operatorname{deg}^{F} v_{2}=b(12)$, and that $\Delta(\Omega)$ contains a face $\Pi_{b}$ such that $\varphi\left(\partial \Pi_{b}\right) \equiv a_{b(3)}^{-\varepsilon_{2} b(4)}$.

For example, if $\tau_{5}(\Delta)=\left|\Delta(0)_{\geq 3}\right|$ and $b(11) \geq 3$, then $\tau_{5}(\Delta)$ is increased by 1 . If $\tau_{6}(\Delta)=\left|\Delta(0)_{\left[k_{6,1}, k_{6,2}\right]}^{F}\right|$ and if $\operatorname{deg}^{F} \alpha(b(2))=b(12)$ satisfies $k_{6,1} \leq b(12) \leq k_{6,2}$, then we increase $\tau_{6}(\Delta)$ by 1 . If $\tau_{2}(\Delta)=\left|\Delta(2)_{a_{1}^{ \pm n_{1}}}\right|$, as above, and $b(3)=1, b(4)=$ $n_{1}$, then we increase $\tau_{2}(\Delta)$ by 1 . If $\tau_{3}(\Delta)=\left|\Delta(2)_{a_{2}^{n_{2}}}\right|$, as above, and $b(3)=2$, $-\varepsilon_{1} b(4)=n_{2}$, then we increase $\tau_{3}(\Delta)$ by 1 . If $\tau_{4}(\Delta):=\sum_{k_{4,1} \leq|\partial \Pi| \leq k_{4,2}}|\partial \Pi|$, as above, and $k_{4,1} \leq b(4) \leq k_{4,2}$, then we increase $\tau_{4}(\Delta)$ by 1 . 
Now we assume that $b(5)=0$, i.e., $b(7)=b(8)=0$, and so $b(4)=1$. Then, to define $b$, we again use formulas (8.9) - (8.10). We also update the tuple $\tau(\Delta)$ according to the information that $\Delta(\Omega)$ contains a face $\Pi_{b}$ with $\varphi\left(\partial \Pi_{b}\right) \equiv a_{b(3)}^{-\varepsilon_{2}}$.

The case of an extension of type 2 on the right is complete.

Suppose that a pseudobracket $b^{\prime} \in B_{i+1}, i \geq 1$, results from $b \in B_{i}$ by an extension of type 3. Denote $a\left(b^{\prime}\right)=e_{1} a(b) e_{2}$, where $a\left(b^{\prime}\right), a(b)$ are the $\operatorname{arcs}$ of $b^{\prime}, b$, resp., and $e_{1}, e_{2}$ are edges of $P_{W}, \varphi\left(e_{1}\right)=\varphi\left(e_{2}\right)^{-1}=a_{j}^{\varepsilon}, \varepsilon= \pm 1$. Recall that both $b$ and $b^{\prime}$ have type PB1. Then we set

$$
\begin{aligned}
& b^{\prime}(7):=0, \quad b^{\prime}(8):=0, \quad b^{\prime}(9):=1, \\
& b^{\prime}(10):=0, \quad b^{\prime}(11):=0, \quad b^{\prime}(12):=0 .
\end{aligned}
$$

We also update the tuple $\tau(\Delta)$ according to the information that $v=\alpha(b(1))=$ $\alpha(b(2))$ is a vertex of $\Delta(\Omega)$ (which is still under construction) such that $\operatorname{deg} v=$ $b(9)+1$ and $\operatorname{deg}^{F} v=b(10)$.

For example, if $\tau_{5}(\Delta)=\left|\Delta(0)_{\geq 3}\right|$ and $b(9) \geq 3$, then $\tau_{5}(\Delta)$ is increased by 1 . If $\tau_{6}(\Delta)=\left|\Delta(0)_{\left[k_{6,1}, k_{6,2}\right]}^{F}\right|$ and if $\operatorname{deg}^{F} v=b(10)$ satisfies $k_{6,1} \leq b(10) \leq k_{6,2}$, then we increase $\tau_{6}(\Delta)$ by 1 .

The case of an extension of type 3 is complete.

Assume that a pseudobracket $b^{\prime} \in B_{i+1}, i \geq 1$, is obtained from $b \in B_{i}$ by a turn operation. Recall that $b$ has type PB1 and $b^{\prime}$ has type PB2. Then we set

$$
\begin{aligned}
& b^{\prime}(7):=0, \quad b^{\prime}(8):=0, \quad b^{\prime}(9):=b(9)+2, \\
& b^{\prime}(10):=b(10)+1, \quad b^{\prime}(11):=0, \quad b^{\prime}(12):=0 .
\end{aligned}
$$

No change over $\tau(\Delta)$ is necessary under a turn operation. The case of a turn operation is complete.

Suppose that a pseudobracket $b^{\prime} \in B_{i+1}, i \geq 1$, is obtained from $b, c \in B_{i}$ by a merger operation. Without loss of generality, we assume that the pseudobrackets $b, c$ which satisfy the condition $b(2)=c(1)$, i.e., $b$ is on the left of $c$. Recall that one of $b, c$ must have type PB1 and the other one has type PB1 or PB2. Consider three cases corresponding to the types of the pseudobrackets $b, c$.

First assume that both $b, c$ have type PB1. Then we set

$$
\begin{array}{ll}
b^{\prime}(7):=0, \quad b^{\prime}(8):=0, & b^{\prime}(9):=b(9)+c(9), \\
b^{\prime}(10):=b(10)+c(10), & b^{\prime}(11):=0, \quad b^{\prime}(12):=0 .
\end{array}
$$

No change over $\tau(\Delta)$ is made.

Assume that $b$ has type PB1 and $c$ has type PB2. Then, keeping in mind that $b(2)=c(1)$, we set

$$
\begin{aligned}
& b^{\prime}(7):=c(7), \quad b^{\prime}(8):=c(8), \quad b^{\prime}(9):=b(9)+c(9), \\
& b^{\prime}(10):=b(10)+c(10), \quad b^{\prime}(11):=c(11), \quad b^{\prime}(12):=c(12) .
\end{aligned}
$$

As above, no change over $\tau(\Delta)$ is necessary.

Assume that $b$ has type PB2 and $c$ has type PB1. If $b(5) \neq 0$, then we set

$$
\begin{aligned}
& b^{\prime}(7):=b(7), \quad b^{\prime}(8):=b(8), \quad b^{\prime}(9):=b(9), \\
& b^{\prime}(10):=b(10), \quad b^{\prime}(11):=b(11)+c(9), \quad b^{\prime}(12):=b(12)+c(10) .
\end{aligned}
$$


On the other hand, if $b(5)=0$, i.e., $b(7)=b(8)=0$, then we set

$$
\begin{array}{ll}
b^{\prime}(7):=0, \quad b^{\prime}(8):=0, & b^{\prime}(9):=b(9)+c(9), \\
b^{\prime}(10):=b(10)+c(10), & b^{\prime}(11):=0, \quad b^{\prime}(12):=0 .
\end{array}
$$

As before, no change over $\tau(\Delta)$ is made under a merger operation.

The case of a merger operation is complete.

Our inductive definitions of extended pseudobrackets and modifications of $\tau(\Delta)$ are complete. To summarize, we conclude that the described changes to the extended pseudobrackets and to the tuple $\tau(\Delta)$ will guarantee that, if our nondeterministic algorithm, which is based on Lemma 4.8 and which follows a sequence $\Omega$ of elementary operations as above, accepts a pair of configurations $\psi_{0}, \psi_{F}$, where $\psi_{0}$ is the empty pseudobracket system and $\psi_{F}$ is a final pseudobracket system, then the final tuple $\tau(\Delta)$, i.e., the tuple associated with $\psi_{F}$, will be equal to the tuple $\tau(\Delta(\Omega))$ which represents the tuple of actual parameters $\tau_{1}(\Delta(\Omega)), \ldots, \tau_{s}(\Delta(\Omega))$ of the diagram $\Delta(\Omega)$.

Consider augmented configurations $\bar{\psi}=(B, \tau(\Delta))$, corresponding to a sequence $\Omega$ of elementary operations as above, where $B$ is a system of extended pseudobrackets and $\tau(\Delta)$ is the tuple associated with $B$. Recall that, by Lemma 4.8, we may assume that $\Omega$ has size bounded by $(11|W|, C(\log |W|+1))$. As in the proof of Theorem 1.2. we note that the entries $b(1), \ldots,|b(5)|, b(6)$ are nonnegative and bounded by $\max (|W|, m)$. It follows from the definitions and Lemma 2.2 (a) that for the new entries $b(7), \ldots, b(12)$, we have that $|b(7)|,|b(8)| \leq|b(5)| \leq|W|$ and

$$
0 \leq b(9), b(10), b(11), b(12) \leq 2|W| .
$$

By the definition, every entry $\tau_{i}(\Delta)$ in $\tau(\Delta)$ satisfies $\left|\tau_{i}(\Delta)\right| \leq C_{\tau}|W|, i=1, \ldots, s_{\tau}$. Since $s_{\tau}, C_{\tau}$ are constants, we conclude that the space needed to store an augmented configuration $\bar{\psi}=(B, \tau(\Delta))$ is $O\left((\log |W|)^{2}\right)$. Therefore, utilizing Savitch's algorithm as before, which now applies to augmented configurations of the form $\bar{\psi}=(B, \tau(\Delta))$, we will be able to find out, in deterministic space $O\left((\log |W|)^{3}\right)$, whether the algorithm accepts a pair $\left(B_{0}, \tau^{0}(\Delta)\right),\left(B_{F}, \tau^{F}(\Delta)\right)$, where $B_{0}$ is empty, $\tau^{0}(\Delta)$ consists of all zeros, $B_{F}$ is a final pseudobracket system, and $\tau^{F}(\Delta)$ is a final tuple corresponding to $B_{F}$. Since the space needed to store a final configuration $\left(B_{F}, \tau^{F}(\Delta)\right)$ is $O(\log |W|)$, we will be able to compute, in deterministic space $O\left((\log |W|)^{3}\right)$, a lexicographically smallest tuple $\left.\widehat{\tau}^{F}(\Delta)\right)$ relative to the property that the pair of augmented configurations $\left(B_{0}, \tau^{0}(\Delta)\right),\left(\widehat{B}_{F}, \widehat{\tau}^{F}(\Delta)\right)$, where $\widehat{B}_{F}$ is some final pseudobracket system, is accepted by Savitch's algorithm. Now we can do the same counter trick as in the beginning of this proof, see (8.3), to compute, in deterministic space $O\left((\log |W|)^{3}\right)$, a sequence $\Omega^{*}$ of elementary operations and the corresponding to $\Omega^{*}$ sequence of augmented configurations which turns $\left(B_{0}, \tau^{0}(\Delta)\right)$ into $\left(\widehat{B}_{F}, \widehat{\tau}^{F}(\Delta)\right)$ and which is constructed by Savitch's algorithm. Finally, as in the beginning of the proof of Theorem 1.5, using the sequence $\Omega^{*}$, we can construct a desired diagram $\Delta^{*}=\Delta\left(\Omega^{*}\right)$ so that $\tau\left(\Delta^{*}\right)=\widehat{\tau}^{F}(\Delta)$ and this construction can be done in deterministic space $O\left((\log |W|)^{3}\right)$. This completes the proof of the space part of Theorem 1.5 .

To prove the time part of Theorem 1.5, we review the proof of $\mathrm{P}$ part of Theorem 1.2. We observe that our arguments enable us, concurrently with computation of the number $\mu_{2}(W[i, j, k, l])$ for every parameterized word $W[i, j, k, l] \in \mathcal{S}_{2}(W)$ 
such that $W(i, j) a_{k}^{\ell} \stackrel{\underline{G}_{2}}{=} 1$, to inductively construct a minimal diagram $\Delta[i, j, k, l]$ over (1.2) such that $\varphi\left(\left.\partial\right|_{0} \Delta[i, j, k, l]\right) \equiv W(i, j) a_{k}^{\ell}$. Details of this construction are straightforward in each of the subcases considered in the proof of $\mathrm{P}$ part of Theorem 1.2. Note that the time needed to run this extended algorithm is still $O\left(|W|^{4} \log |W|\right)$.

Theorem 1.5 is proven.

Corollary 1.6. There is a deterministic algorithm that, for given word $W$ over the alphabet $\mathcal{A}^{ \pm 1}$ such that $W^{\stackrel{\mathcal{F}(\mathcal{A})}{=}} 1$, where $\mathcal{F}(\mathcal{A})=\langle\mathcal{A} \| \varnothing\rangle$ is the free group over $\mathcal{A}$, constructs a pattern of cancellations of letters in $W$ that result in the empty word and the algorithm operates in space $O\left((\log |W|)^{3}\right)$.

Furthermore, let $\Delta$ be a disk diagram over $\mathcal{F}(\mathcal{A})$ that corresponds to a pattern of cancellations of letters in $W$, i.e., $\varphi(\partial \Delta) \equiv W$, and let

$$
\tau(\Delta)=\left(\tau_{1}(\Delta), \ldots, \tau_{s_{\tau}}(\Delta)\right)
$$

be a tuple of integers, where the absolute value $\left|\tau_{i}(\Delta)\right|$ of each $\tau_{i}(\Delta)$ represents the number of vertices in $\Delta$ of certain degree. Then, also in deterministic space $O\left((\log |W|)^{3}\right)$, one can algorithmically construct such a diagram $\Delta$ which is smallest relative to the tuple $\tau(\Delta)$.

Proof. Corollary 1.6 is immediate from Theorem 1.5 and does not need a separate proof. Nevertheless, it is worth mentioning that, in the case of presentation $\mathcal{F}(\mathcal{A})=\langle\mathcal{A} \| \varnothing\rangle$ of the free group $\mathcal{F}(\mathcal{A})$ over $\mathcal{A}$, our definitions of brackets, pseudobrackets, elementary operations and subsequent arguments become significantly simpler. Since there are no relations, we do not define brackets of type B2, nor we define pseudobrackets of type PB2. In particular, for every bracket or pseudobracket $b$, the entries $b(3), b(4), b(5)$ are always zeroes and could be disregarded. In the extended version of a pseudobracket $b$, defined for minimization of $\Delta$ relative to $\tau(\Delta)$, the entries $b(7), b(8), b(10), b(12)$ are also always zeroes and could be disregarded. Furthermore, there is no need to consider extensions of type 1, 2 and turn operations. Hence, in this case, we only need elementary operations which are additions, extensions of type 3 and mergers over brackets of type B1, pseudobrackets of type PB1, and over their systems.

\section{Construction of Minimal Diagrams over (1.4) and Proof of THEOREM 1.7}

Theorem 1.7. Suppose that $W$ is a word over the alphabet $\mathcal{A}^{ \pm 1}$ such that the bounded word problem for presentation (1.4) holds for the pair $(W, n)$. Then a minimal diagram $\Delta$ over (1.4) such that $\varphi(\partial \Delta) \equiv W$ can be algorithmically constructed in deterministic space $O\left(\max (\log |W|, \log n)(\log |W|)^{2}\right)$ or in deterministic time $O\left(|W|^{4}\right)$.

In addition, if $\left|n_{1}\right|=\left|n_{2}\right|$ in (1.4), then the minimal diagram problem for presentation (1.4) can be solved in deterministic space $O\left((\log |W|)^{3}\right)$ or in deterministic time $O\left(|W|^{3} \log |W|\right)$.

Proof. First we prove the space part of Theorem 1.7

Let $W$ be a nonempty word over $\mathcal{A}^{ \pm 1}$ such that $W \stackrel{\mathcal{G}_{3}}{=} 1$, where $\mathcal{G}_{3}$ is defined by presentation (1.4), and there is a disk diagram $\Delta$ over (1.4) such that $\varphi(\partial \Delta) \equiv W$ and $|\Delta(2)| \leq n$, i.e., the bounded word problem has a positive solution for the pair $(W, n)$. 
It follows from Lemma 6.13 that there is a finite sequence $\Omega$ of elementary operations such that $\Omega$ converts the empty pseudobracket system for $W$ into a final one and $\Omega$ has other properties stated in Lemma 6.13. As in the proof of Theorem 1.4. Lemma 6.13 gives us a nondeterministic algorithm which runs in time $O(|W|)$ and space $O((\max (\log |W|, \log n) \log |W|)$ and which accepts a word $W$ over $\mathcal{A}^{ \pm 1}$ if and only if the bounded word problem has a positive solution for the pair $(W, n)$. Note that here the big- $O$ constants can be written down explicitly (see the proof of Theorem 1.4).

Furthermore, as in the proof of Theorem 1.4 using Savitch's theorem [38, see also [3], 32, we obtain a deterministic algorithm which runs in space

$$
O\left(\left(\max (\log |W|, \log n)(\log |W|)^{2}\right)\right.
$$

and which computes a minimal integer $n(W), 0 \leq n(W) \leq n$, such that there is a disk diagram $\Delta$ over (1.4) so that $\varphi(\partial \Delta) \equiv W$ and $|\Delta(2)|=n(W)$. To do this, we can check by Savitch's algorithm whether the empty pseudobracket system $B_{0}$ can be transformed by elementary operations into a final pseudobracket system $\left\{b_{F}\right\}$, where $b_{F}(4)=n^{\prime}$ for $n^{\prime}=0,1,2, \ldots, n$.

Without loss of generality, we may assume that $n(W)>0$ because if $n(W)=0$ then Corollary 1.6 yields the desired result.

Having found this number $n(W) \geq 1$ in deterministic space (9.1), we will run Savitch's algorithm again for the pair $B_{0}$ and $\left\{b_{F}^{*}\right\}$, where $b_{F}^{*}=(0,|W|, 0, n(W))$, and use the counter trick, as in the proof of Theorem 1.5 to compute an instance of a sequence $\Omega$ of elementary operations and the corresponding to $\Omega$ sequence $B_{0}, B_{1}, \ldots, B_{\ell}=\left\{b_{F}^{*}\right\}$ of pseudobracket systems. After computing these sequences $\Omega$ and $B_{0}, B_{1}, \ldots, B_{\ell}=\left\{b_{F}^{*}\right\}$, our algorithm halts. Denote this modification of Savitch's algorithm by $\mathfrak{A}_{n}$.

Denote

$$
\Omega=\left(\omega_{1}, \ldots, \omega_{\ell}\right)
$$

where $\omega_{1}, \ldots, \omega_{\ell}$ are elementary operations and, as above, let $B_{0}, B_{1}, \ldots, B_{\ell}$ be the corresponding to $\Omega$ sequence of pseudobracket systems so that $B_{j}$ is obtained by application of $\omega_{j}$ to $B_{j-1}$, so $B_{j}=\omega_{j}\left(B_{j-1}\right)$. We also let

$$
\left(\chi_{1}, \ldots, \chi_{\ell_{2}}\right)
$$

denote the subsequence of $\Omega$ that consists of all extensions of type 2 . Also, let $c_{i} \in B_{j_{i}-1}$ denote the pseudobracket to which the elementary operation $\chi_{i}$ applies and let $d_{i} \in B_{j_{i}}$ denote the pseudobracket obtained from $c_{i}$ by application of $\chi_{i}$, so $d_{i}=\chi_{i}\left(c_{i}\right)$.

According to the proof of Lemma 6.12, the sequence $\Omega$ or, equivalently, the sequence $B_{0}, B_{1}, \ldots, B_{\ell}$, defines a disk diagram $\Delta(\Omega)$ which can be inductively constructed as in the proof of Claim (D1). Furthermore, according to the proof of Claim (D1), all faces of $\Delta(\Omega)$ are contained in $\ell_{2} a_{2}$-bands $\Gamma_{1}, \ldots, \Gamma_{\ell_{2}}$ which are in bijective correspondence with elementary operations $\chi_{1}, \ldots, \chi_{\ell_{2}}$ so that $\Gamma_{i}$ corresponds to $\chi_{i}, i=1, \ldots, \ell_{2}$. Denote

$$
\partial \Gamma_{i}=f_{i} t_{i} g_{i} u_{i}
$$


where $f_{i}, g_{i}$ are edges of $\partial \Delta(\Omega), \varphi\left(f_{i}\right)=\varphi\left(g_{i}\right)^{-1} \in\left\{a_{2}^{ \pm 1}\right\}$, and $t_{i}, u_{i}$ are simple paths whose labels are powers of $a_{1}$. Hence,

$$
\sum_{i=1}^{\ell_{2}}\left|\Gamma_{i}(2)\right|=|\Delta(\Omega)(2)|=n(W) .
$$

It follows from the definitions that if $a\left(c_{i}\right), a\left(d_{i}\right)$ are the arcs of the pseudobrackets $c_{i}, d_{i}$, resp., and $e_{1, i} a\left(c_{i}\right) e_{2, i}$ is a subpath of the path $P_{W}$, where $e_{1, i}, e_{2, i}$ are edges of $P_{W}$, then, renaming $f_{i} \leftrightarrows g_{i}, t_{i} \leftrightarrows u_{i}$ if necessary, we have the following equalities

$$
\begin{array}{cl}
\alpha\left(e_{1, i}\right)=f_{i}, & \alpha\left(e_{2, i}\right)=g_{i}, \quad \varphi\left(f_{i}\right)=\varphi\left(g_{i}\right)^{-1}=a_{2}^{\varepsilon_{i}}, \quad \varepsilon_{i}= \pm 1, \\
\varphi\left(t_{i}\right) \equiv a_{1}^{c_{i}(3)}, & \varphi\left(u_{i}\right) \equiv a_{1}^{-d_{i}(3)}, \quad \alpha\left(a\left(c_{i}\right)\right)=t_{i}, \quad \alpha\left(a\left(d_{i}\right)\right)=u_{i}^{-1}
\end{array}
$$

for every $i=1, \ldots, \ell_{2}$, see Fig. 9.1.

$$
f_{i}=\alpha\left(e_{1, i}\right)\left\{\begin{array}{c}
t_{i}=\alpha\left(a\left(c_{i}\right)\right) \\
\Gamma_{i} \\
u_{i}=\alpha\left(a\left(d_{i}\right)\right)^{-1}
\end{array} g_{i}=\alpha\left(e_{2, i}\right)\right.
$$

Fig. 9.1

Note that each of these $a_{2}$-bands $\Gamma_{1}, \ldots, \Gamma_{\ell_{2}}$ can be constructed, as a part of the sequence $\Omega$, in deterministic space (9.1) by running the algorithm $\mathfrak{A}_{n}$. For instance, if we wish to retrieve information about the diagram $\Gamma_{i}$, we would be looking for the $i$ th extension of type 2 in the sequence $\Omega$, denoted above by $\chi_{i}$. We also remark that the parameters $\left(c_{i}, \varepsilon_{i}\right)$, associated with the elementary operation $\chi_{i}$, contain all the information about the diagram $\Gamma_{i}$.

Observe that we are not able to keep all these pairs $\left(c_{i}, \varepsilon_{i}\right), i=1, \ldots, \ell_{2}$, in our intermediate computations aimed to construct $\Delta(\Omega)$ in polylogarithmic space because doing this would take polynomial space. However, we can reuse space, so we keep one pair $\left(c_{i}, \varepsilon_{i}\right)$, or a few pairs, in memory at any given time and, when we need a different pair $\left(c_{j}, \varepsilon_{j}\right)$, we erase $\left(c_{i}, \varepsilon_{i}\right)$ and compute the new pair $\left(c_{j}, \varepsilon_{j}\right)$ by running the algorithm $\mathfrak{A}_{n}$ as discussed above.

We can also output all these pairs $\left(c_{i}, \varepsilon_{i}\right), i=1, \ldots, \ell_{2}$, as a part of our description of the disk diagram $\Delta(\Omega)$ still under construction.

Thus, in deterministic space (9.1), we have obtained the information about $a_{2^{-}}$ bands $\Gamma_{1}, \ldots, \Gamma_{\ell_{2}}$ of $\Delta(\Omega)$ that contain all of the faces of $\Delta(\Omega)$ and it remains to describe, working in space (9.1), how the edges of $(\partial \Delta(\Omega))^{ \pm 1}$ and those of $\left(\partial \Gamma_{1}\right)^{ \pm 1}, \ldots,\left(\partial \Gamma_{\ell_{2}}\right)^{ \pm 1}$ are attached to each other.

Observe that by taking the subdiagrams $\Gamma_{1}, \ldots, \Gamma_{\ell_{2}}$ out of $\Delta(\Omega)$, we will produce $\ell_{2}+1$ connected components $\Delta_{1}, \ldots, \Delta_{\ell_{2}+1}$ which are disk diagrams with no faces, i.e., $\Delta_{1}, \ldots, \Delta_{\ell_{2}+1}$ are disk diagrams over the free group $F(\mathcal{A})=\langle\mathcal{A} \| \varnothing\rangle$. Note that the boundary of each disk diagram $\Delta_{i}$ has a natural factorization

$$
\partial \Delta_{i}=q_{1, i} r_{1, i} \ldots q_{k_{i}, i} r_{k_{i}, i}
$$

where every $q_{j, i}$ is a subpath of the cyclic path $\partial \Delta(\Omega)$, perhaps, $\left|q_{j, i}\right|=0$, and every $r_{j, i}$ is one of the paths $t_{1}^{-1}, u_{1}^{-1}, \ldots, t_{\ell_{2}}^{-1}, u_{\ell_{2}}^{-1},\left|r_{j, i}\right|>0$, see Fig. 9.2. 


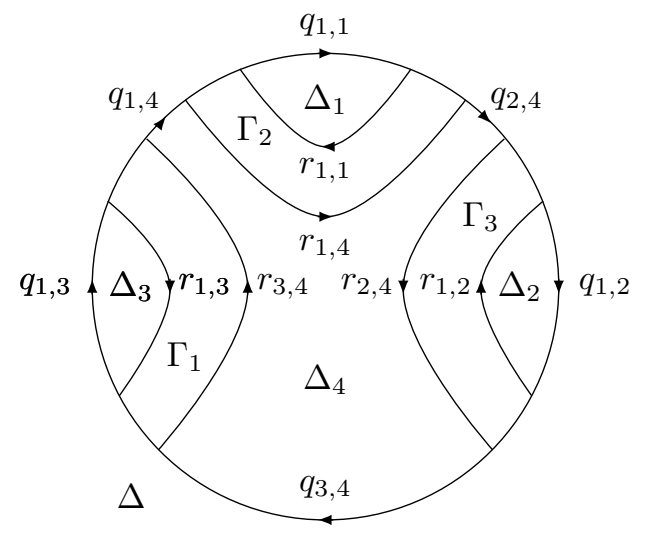

Fig. 9.2

It follows from the definitions and Lemma 6.13 that

$$
\sum_{i=1}^{\ell_{2}}\left|\partial \Delta_{i}\right|<|\partial \Delta(\Omega)|+|\Delta(\Omega)(2)|\left(\left|n_{1}\right|+\left|n_{2}\right|\right)=O(\max (|W|, n(W))),
$$

as $|\Delta(\Omega)(2)|=n(W)$ and $|\partial \Delta(\Omega)|=|W|$.

Suppose that a vertex $v \in P_{W}$ is given, $0 \leq v \leq\left|P_{W}\right|=|W|$. Then there is a unique disk diagram $\Delta_{i_{v}}$ among $\Delta_{1}, \ldots, \Delta_{\ell_{2}+1}$ such that $\alpha(v) \in \partial \Delta_{i_{v}}$. We now describe an algorithm $\mathfrak{B}_{v}$ that, for an arbitrary edge $e$ of the boundary $\partial \Delta_{i_{v}}$, computes in deterministic space (9.1) the label $\varphi(e)$ of $e$ and the unique location of $e$ in one of the paths $\left.\partial\right|_{0} \Delta(\Omega), t_{1}^{-1}, u_{1}^{-1}, \ldots, t_{\ell_{2}}^{-1}, u_{\ell_{2}}^{-1}$. To do this, we will go around the boundary path $\left.\partial\right|_{\alpha(v)} \Delta_{i_{v}}$, starting at the vertex $\alpha(v)$. Initializing the parameters $v^{*}, d^{*}$, we set

$$
v^{*}:=v, \quad d^{*}:=1 \text {. }
$$

If $e$ is the $k$ th edge of $\left.\partial\right|_{\alpha(v)} \Delta_{i_{v}}, 1 \leq k \leq\left|\partial \Delta_{i_{v}}\right|$, then we also say that $e$ is the edge of $\left.\partial\right|_{\alpha(v)} \Delta_{i_{v}}$ with number $k$.

Let $e_{c}$ denote the edge of $P_{W}$ such that $\left(e_{c}\right)_{-}=v^{*}$ if $v^{*}<|W|$ and $\left(e_{c}\right)_{-}=0$ if $v^{*}=|W|$. We now consider three possible Cases 1-3.

Case 1. Assume that $\alpha\left(e_{c}\right)=f_{i}$ for some $i=1, \ldots, \ell_{2}$, see the definition (9.2) of $\partial \Gamma_{i}$. Note that such an equality $\alpha\left(e_{c}\right)=f_{i}$ can be verified in space (9.1) by checking, one by one, all $a_{2}$-bands $\Gamma_{1}, \ldots, \Gamma_{\ell_{2}}$.

If $v^{*}=v$, then the first $\left|t_{i}\right|$ edges of the boundary path

$$
\left.\partial\right|_{\alpha(v)} \Delta_{i_{v}}=t_{i}^{-1} \ldots
$$

are the edges of the path $t_{i}^{-1}$, see Fig. 9.3(a).

In the general case when $v^{*}$ is arbitrary, we obtain that the edges of the boundary path $\left.\partial\right|_{\alpha(v)} \Delta_{i_{v}}$ with numbers $d^{*}, \ldots, d^{*}+\left|t_{i}\right|-1$ are consecutive edges of the path $t_{i}^{-1}$ starting from the first one.

Let $v^{\prime} \in P_{W}$ denote the vertex such that $\alpha\left(v^{\prime}\right)=\left(t_{i}\right)_{-}$, see Fig. 9.3(a). Also, denote $d^{\prime}:=d^{*}+\left|t_{i}\right|$.

Case 2. Assume that $\alpha\left(e_{c}\right)=g_{i}$ for some $i=1, \ldots, \ell_{2}$, see (9.2). As in Case 1, we can verify this equality in space (9.1). 
If $v^{*}=v$, then the first $\left|u_{i}\right|$ edges of the boundary path

$$
\left.\partial\right|_{\alpha(v)} \Delta_{i_{v}}=u_{i}^{-1} \ldots
$$

are the edges of the path $u_{i}^{-1}$, see Fig. 9.3(b).

In the general case when $v^{*}$ is arbitrary, we obtain that the edges of the boundary path $\left.\partial\right|_{\alpha(v)} \Delta_{i_{v}}$ with numbers $d^{*}, \ldots, d^{*}+\left|u_{i}\right|-1$ are consecutive edges of the path $u_{i}^{-1}$ starting from the first one.

Let $v^{\prime} \in P_{W}$ denote the vertex such that $\alpha\left(v^{\prime}\right)=\left(u_{i}\right)_{-}$, see Fig. 9.3(b). Also, denote $d^{\prime}:=d^{*}+\left|u_{i}\right|$.

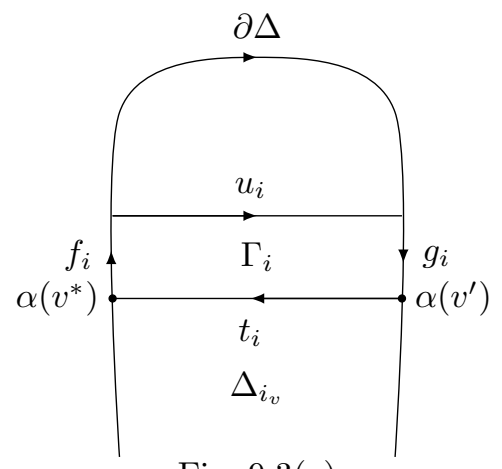

Fig. 9.3(a)

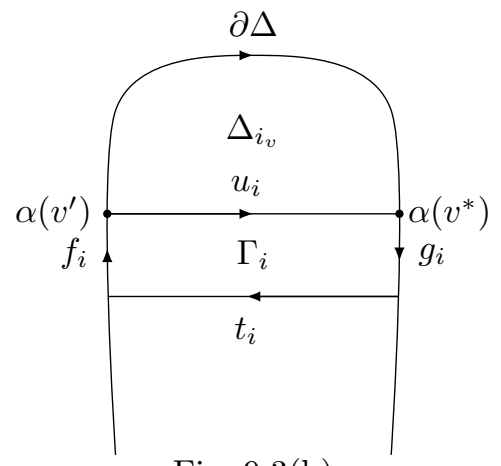

Fig. 9.3(b)

Case 3. Suppose that $\alpha\left(e_{c}\right)$ is not one of the edges $f_{i}, g_{i}$ of $a_{2}$-bands $\Gamma_{1}, \ldots, \Gamma_{\ell_{2}}$. As above, we can verify this claim in space (9.1).

If $v^{*}=v$, then the first edge of the boundary path

$$
\left.\partial\right|_{\alpha(v)} \Delta_{i_{v}}=\alpha\left(e_{c}\right) \ldots
$$

is $\alpha\left(e_{c}\right)$.

In the general case when $v^{*}$ is arbitrary, we have that the edge of the boundary path $\left.\partial\right|_{\alpha(v)} \Delta_{i_{v}}$ with number $d^{*}$ is $\alpha\left(e_{c}\right)$, see Fig. 9.4.

Denote $v^{\prime}:=\left(e_{c}\right)_{-}$and let $d^{\prime}:=d^{*}+1$, see Fig. 9.4.

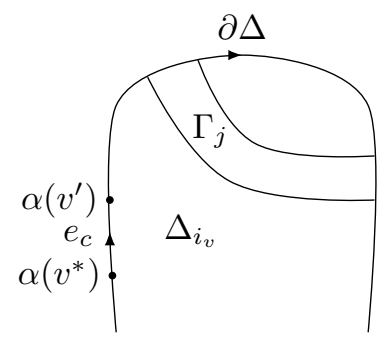

Fig. 9.4

The foregoing mutually exclusive Cases $1-3$ describe a cycle, including the first one when $v^{*}=v$, of the algorithm $\mathfrak{B}_{v}$ which is finished by reassignment $v^{*}:=v^{\prime}$ and $d^{*}:=d^{\prime}$.

We now repeat the above cycle with new values of parameters $v^{*}, d^{*}$, whose storage along with storage of the original vertex $v$ requires additional

$$
O(\max (\log n, \log |W|))
$$


space, as follows from the definitions and inequality (9.4). We keep on repeating this cycle until we obtain at certain point that the vertex $v^{*}$ is again $v$ which means that all the edges of the path $\left.\partial\right|_{\alpha(v)} \Delta_{i_{v}}$ have been considered, we are back at $v$ and we stop. Note that $d^{*}=|\partial|_{\alpha(v)} \Delta_{i_{v}} \mid$ when we stop.

We remark that, when running the algorithm $\mathfrak{B}_{v}$ we can stop at once and abort computations whenever we encounter the value of parameter $v^{*}$ less than $v$, because, in this case, the disk diagram $\Delta_{i_{v}}$ which contains the vertex $v$ is identical to $\Delta_{i_{v^{*}}}$ and the information about the edges of $\Delta_{i_{v^{*}}}$ can be obtained by running the algorithm $\mathfrak{B}_{v^{*}}$ instead. Thus, we now have that either the algorithm $\mathfrak{B}_{v}$ aborts at some point or $\mathfrak{B}_{v}$ performs several cycles and stops when $v=v^{*}$ and $d^{*}>1$, in which case we say that the algorithm $\mathfrak{B}_{v}$ accepts the diagram $\Delta_{i_{v}}$.

By running the algorithms $\mathfrak{B}_{v}$ consecutively for $v=0,1, \ldots$, with possible stops as described above, we will get the information about the edges of all disk diagrams $\Delta_{1}, \ldots, \Delta_{\ell_{2}+1}$ so that for every $j=1, \ldots, \ell_{2}+1$, there will be a unique vertex $v(j) \in P_{W}$ such that $\Delta_{j}=\Delta_{i_{v(j)}}$ and the algorithm $\mathfrak{B}_{v(j)}$ accepts $\Delta_{j}$. Recall that, in the proof of Theorem 1.2, see also Corollary 1.6, we constructed a deterministic algorithm $\mathfrak{C}$ such that, when given a word $U$ so that $U=1$ in the free group $F(\mathcal{A})$, the algorithm $\mathfrak{C}$ outputs a diagram $\Delta_{U}$ over $F(\mathcal{A})=\langle\mathcal{A} \| \varnothing\rangle$ such that $\varphi\left(\left.\partial\right|_{0} \Delta_{U}\right) \equiv U$ and the algorithm $\mathfrak{C}$ operates in space $O\left((\log |U|)^{3}\right)$. Next, we observe that our ability to deterministically compute, in space (9.1), the label $\varphi(e)$ of every edge $e$ of the boundary path $\left.\partial\right|_{\alpha(v)} \Delta_{i_{v}}$, as well as the location of $e$ in one of the paths $\left.\partial\right|_{0} \Delta(\Omega), t_{1}^{-1}, u_{1}^{-1}, \ldots, t_{\ell_{2}}^{-1}, u_{\ell_{2}}^{-1}$, combined together with the algorithm $\mathfrak{C}$, means that we can construct a disk diagram $\widetilde{\Delta}_{i_{v}}$ over $F(\mathcal{A})$ such that $\varphi\left(\widetilde{\Delta}_{i_{v}}\right) \equiv \varphi\left(\Delta_{i_{v}}\right)$ in deterministic space

$$
O\left(\left|\partial \Delta_{i_{v}}\right|^{3}\right)=O\left(\left(\max (\log |W|, \log n(W))^{3}\right)\right),
$$

see (9.4). Replacing the disk diagrams $\Delta_{1}, \ldots, \Delta_{\ell_{2}+1}$ in $\Delta(\Omega)$ with their substitutes $\widetilde{\Delta}_{1}, \ldots, \widetilde{\Delta}_{\ell_{2}+1}$, we will obtain a disk diagram $\widetilde{\Delta}(\Omega)$ over (1.4) such that

$$
\varphi(\partial \widetilde{\Delta}(\Omega)) \equiv \varphi(\partial \Delta(\Omega)), \quad|\widetilde{\Delta}(\Omega)(2)|=|\Delta(\Omega)(2)|=n(W),
$$

i.e., $\widetilde{\Delta}(\Omega)$ is as good as $\Delta(\Omega)$.

Since the disk diagrams $\widetilde{\Delta}_{1}, \ldots, \widetilde{\Delta}_{\ell_{2}+1}$ along with $a_{2}$-bands $\Gamma_{1}, \ldots, \Gamma_{\ell_{2}}$ constitute the entire diagram $\widetilde{\Delta}(\Omega)$, our construction of $\widetilde{\Delta}(\Omega)$, performed in space (9.1), is now complete.

It remains to prove the additional claim of Theorem 1.7. We start with the following.

Lemma 9.1. Suppose $\Delta_{0}$ is a reduced disk diagram over (1.4), where $\left|n_{1}\right|=\left|n_{2}\right|$. Then the number $\left|\Delta_{0}(2)\right|$ of faces in $\Delta_{0}$ satisfies $\left|\Delta_{0}(2)\right| \leq \frac{1}{4\left|n_{1}\right|}\left|\partial \Delta_{0}\right|^{2}$.

Proof. Denote $n_{0}:=\left|n_{1}\right|$ and set $n_{2}=\kappa n_{1}$, where $\kappa= \pm 1$. Consider the presentation

$$
\mathcal{G}_{4}=\left\langle\mathcal{A}, b_{1}, \ldots, b_{n_{0}-1} \| a_{2} a_{1} b_{1}^{-1}=a_{1}^{\kappa}, b_{1} a_{1} b_{2}^{-1}=a_{1}^{\kappa}, \ldots, b_{n_{0}-1} a_{1} a_{2}^{-1}=a_{1}^{\kappa}\right\rangle
$$

that has $n_{0}$ relations which are obtained by splitting the relation $a_{2} a_{1}^{n_{1}} a_{2}^{-1}=a_{1}^{\kappa n_{1}}$ of (1.4) into $n_{0}$ "square" relations, see Fig. 9.5 where the case $n_{0}=3$ is depicted. 


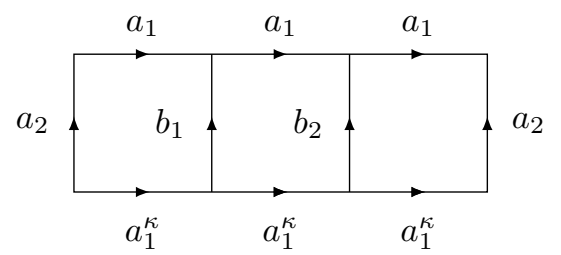

Fig. 9.5

Note that there is a natural isomorphism $\psi: \mathcal{G}_{4} \rightarrow \mathcal{G}_{3}$ defined by the map $\psi(a)=a$ for $a \in \mathcal{A}$, and $\psi\left(b_{j}\right)=a_{1}^{-\kappa j} a_{2} a_{1}^{j}$ for $j=1, \ldots, n_{0}-1$.

If $\Delta_{0}$ is a reduced disk diagram over (1.4), then we can split faces of $\Delta_{0}$ into "square" faces over (9.5), as in Fig. 9.5, and obtain a reduced disk diagram $\Delta_{0, b}$ over (9.5) such that

$$
\varphi\left(\partial \Delta_{0, b}\right) \equiv \varphi\left(\partial \Delta_{0}\right), \quad\left|\Delta_{0, b}(2)\right|=n_{0}\left|\Delta_{0}(2)\right| .
$$

Let $\Delta$ be an arbitrary reduced disk diagram over presentation (9.5).

We modify the definition of an $a_{i}$-band given in Sect. 6 for diagrams over presentation (1.4) so that this definition would be suitable for diagrams over (9.5).

Two faces $\Pi_{1}, \Pi_{2}$ in a disk diagram $\Delta$ over (9.5) are called $j$-related, where $j=1,2$, denoted $\Pi_{1} \leftrightarrow_{j} \Pi_{2}$, if there is an edge $e$ such that $e \in \partial \Pi_{1}, e^{-1} \in \partial \Pi_{2}$, and $\varphi(e)=a_{1}^{ \pm 1}$ if $j=1$ or $\varphi(e) \in\left\{a_{2}^{ \pm 1}, b_{1}^{ \pm 1}, \ldots, b_{n_{0}-1}^{ \pm 1}\right\}$ if $j=2$. As before, we consider a minimal equivalence relation, denoted $\sim_{j}$, on the set of faces of $\Delta$ generated by the relation $\leftrightarrow_{j}$.

An $a_{i}$-band, where $i \geq 1$ is now arbitrary, is a minimal subcomplex $\Gamma$ of $\Delta$ that contains an edge $e$ such that $\varphi(e)=a_{i}^{ \pm 1}$ if $i \neq 2$ or $\varphi(e) \in\left\{a_{2}^{ \pm 1}, b_{1}^{ \pm 1}, \ldots, b_{n_{0}-1}^{ \pm 1}\right\}$ if $i=2$ and $\Gamma$ has the following property. If there is a face $\Pi$ in $\Delta$ such that $e \in(\partial \Pi)^{ \pm 1}$, then $\Gamma$ must contain all faces of the equivalence class $[\Pi]_{\sim_{i}}$ of $\Pi$. As before, this definition implies that an $a_{i}$-band $\Gamma$ is either a subcomplex consisting of a single nonoriented edge $\left\{f, f^{-1}\right\}$, where $\varphi(f)=a_{i}^{ \pm 1}$ if $i \neq 2$ or $\varphi(f) \in\left\{a_{2}^{ \pm 1}, b_{1}^{ \pm 1}, \ldots, b_{n_{0}-1}^{ \pm 1}\right\}$ if $i=2$ and $f, f^{-1} \in \partial \Delta$, or $\Gamma$ consists of all faces of an equivalence class $[\Pi]_{\sim_{i}}$, here $i=1,2$.

If an $a_{i}$-band $\Gamma$ contains faces, then $\Gamma$ is called essential.

If an $a_{i}$-band $\Gamma$ is essential but $\Gamma$ contains no face whose boundary contains an edge $f$ such that $f^{-1} \in \partial \Delta$ and $\varphi(f)=a_{1}^{ \pm 1}$ if $i=1$ or $\varphi(f) \in\left\{a_{2}^{ \pm 1}, b_{1}^{ \pm 1}, \ldots, b_{n_{0}-1}^{ \pm 1}\right\}$ if $i=2$, then $\Gamma$ is called a closed $a_{i}$-band. It follows from the definitions that if $\Gamma$ is an essential $a_{i}$-band, then $i=1,2$.

If $\Gamma$ is an essential $a_{i}$-band and $\Pi_{1}, \ldots, \Pi_{k}$ are all faces of $\Gamma$, we consider a simple arc or a simple curve $c(\Gamma)$ in the interior of $\Gamma$ such that the intersection $c(\Gamma) \cap \Pi_{j}$ for every $j=1, \ldots, k$, is a properly embedded arc in $\Pi_{j}$ whose boundary points belong to the interior of different edges of $\partial \Pi_{j}$ whose $\varphi$-labels are in $\left\{a_{1}^{ \pm 1}\right\}$ if $i=1$ or in $\left\{a_{2}^{ \pm 1}, b_{1}^{ \pm 1}, \ldots, b_{n_{0}-1}^{ \pm 1}\right\}$ if $i=2$. This arc or curve $c(\Gamma)$ will be called a connecting line of $\Gamma$.

Note that if $\Gamma$ contains a face $\Pi$ whose boundary has an edge $f$ such that $f^{-1} \in$ $\partial \Delta$ and $\varphi(f)=a_{1}^{ \pm 1}$ if $i=1$ or $\varphi(f) \in\left\{a_{2}^{ \pm 1}, b_{1}^{ \pm 1}, \ldots, b_{n_{0}-1}^{ \pm 1}\right\}$ if $i=2$, then a connecting line $c(\Gamma)$ of $\Gamma$ connects two points $\partial c(\Gamma)$ on the boundary $\partial \Delta$. On the other hand, if $\Gamma$ contains no face $\Pi$ as above, then $c(\Gamma)$ is a closed simple curve, in which case $\Gamma$ is called a closed $a_{i}$-band. 
If $\Pi$ is a face in $\Delta$ over (9.5), then there are exactly two bands, $a_{1}$-band and $a_{2}$-band, denoted $\Gamma_{\Pi, 1}, \Gamma_{\Pi, 2}$, resp., whose connecting lines $c\left(\Gamma_{\Pi, 1}\right), c\left(\Gamma_{\Pi, 2}\right)$ pass through $\Pi$. Without loss of generality, we may assume that the intersection

$$
c\left(\Gamma_{\Pi, 1}\right) \cap c\left(\Gamma_{\Pi, 2}\right) \cap \Pi
$$

consists of a single (piercing) point. The following lemma has similarities with Lemma 6.1

Lemma 9.2. Suppose that $\Delta$ is a reduced disk diagram over presentation (9.5). Then there are no closed $a_{i}$-bands in $\Delta$ and every $a_{i}$-band $\Gamma$ of $\Delta$ is a disk subdiagram of $\Delta$ such that

$$
\left.\partial\right|_{\left(f_{1}\right)_{-}} \Gamma=f_{1} s_{1} f_{2} s_{2},
$$

where $f_{1}, f_{2}$ are edges of $\partial \Delta$ such that

$$
\begin{aligned}
& \varphi\left(f_{1}\right), \varphi\left(f_{2}\right) \in\left\{a_{i}^{ \pm 1}\right\} \quad \text { if } i \neq 2, \\
& \varphi\left(f_{1}\right), \varphi\left(f_{2}\right) \in\left\{a_{2}^{ \pm 1}, b_{1}^{ \pm 1}, \ldots, b_{n_{0}-1}^{ \pm 1}\right\} \quad \text { if } i=2,
\end{aligned}
$$

$s_{1}, s_{2}$ are simple paths such that $\left|s_{1}\right|=\left|s_{2}\right|=|\Gamma(2)|$, and $\partial \Gamma$ is a reduced simple closed path when $|\Gamma(2)|>0$. In addition, if $\Gamma_{1}$ and $\Gamma_{2}$ are essential $a_{1}$-band and $a_{2}$-band, resp., in $\Delta$, then their connecting lines $c\left(\Gamma_{1}\right), c\left(\Gamma_{2}\right)$ intersect in at most one point.

Proof. Since $\Delta$ is reduced, it follows that if $\Pi_{1} \leftrightarrow_{j} \Pi_{2}, j=1,2$, then $\Pi_{1}$ is not a mirror copy of $\Pi_{2}$, i.e., $\varphi\left(\Pi_{1}\right) \not \equiv \varphi\left(\Pi_{2}\right)^{-1}$. This remark, together with the definition of an $a_{i}$-band and defining relations of presentation (9.5), implies that if $\Pi_{1} \leftrightarrow_{j} \Pi_{2}$ then the faces $\Pi_{1}, \Pi_{2}$ share exactly one nonoriented edge. This, in particular, implies $\left|s_{1}\right|=\left|s_{2}\right|=|\Gamma(2)|$ if $\Gamma$ is an $a_{i}$-band such that $\partial \Gamma=f_{1} s_{1} f_{2} s_{2}$ as in the statement of Lemma 9.2 .

Assume, on the contrary, that there is an essential $a_{i}$-band $\Gamma_{0}$ in $\Delta$ such that either $\Gamma_{0}$ is closed or $\partial \Gamma_{0}=f_{1} s_{1} f_{2} s_{2}$ as above, and one of the paths $s_{1}, s_{2}$ is not simple. Then there is a disk subdiagram $\Delta_{2}$ of $\Delta$ bounded by edges of $\partial \Gamma_{0}$ such that $\varphi\left(\partial \Delta_{2}\right)$ is a nonempty reduced word over the alphabet $\left\{a_{1}^{ \pm 1}\right\}$ if $i=2$ or over the alphabet $\left\{a_{2}^{ \pm 1}, b_{1}^{ \pm 1}, \ldots, b_{n_{0}-1}^{ \pm 1}\right\}$ if $i=1$. Pick such diagrams $\Gamma_{0}$ and $\Delta_{2}$ so that $\left|\Delta_{2}(2)\right|$ is minimal. Since $\Delta_{2}$ contains faces and $\varphi\left(\partial \Delta_{2}\right)$ contains no letters either from $\left\{a_{1}^{ \pm 1}\right\}$ if $i=1$ or from $\left\{a_{2}^{ \pm 1}, b_{1}^{ \pm 1}, \ldots, b_{n_{0}-1}^{ \pm 1}\right\}$ if $i=2$, it follows that every $a_{3-i}$-band in $\Delta_{2}$ is closed and bounds a similar to $\Delta_{2}$ diagram $\Delta_{2}^{\prime}$ such that $\left|\Delta_{2}^{\prime}(2)\right|<\left|\Delta_{2}(2)\right|$. This contradiction proves that an $a_{i}$-band $\Gamma_{0}$ with the above properties does not exist.

To prove the space part of the additional claim, suppose, on the contrary, that there are essential $a_{1}$ - and $a_{2}$-bands $\Gamma_{1}$ and $\Gamma_{2}$, resp., in $\Delta$ such that the intersection $c\left(\Gamma_{1}\right) \cap c\left(\Gamma_{2}\right)$ of their connecting lines $c\left(\Gamma_{1}\right), c\left(\Gamma_{2}\right)$ contains at least two points. We pick two such consecutive along $c\left(\Gamma_{1}\right), c\left(\Gamma_{2}\right)$ points and let $\Pi_{1}, \Pi_{2}$ be the faces that contain these two points. Then there exists a disk subdiagram $\Delta_{1}$ in $\Delta$ such that $\partial \Delta_{1}=u_{1} u_{2}$, where $u_{1}^{-1}$ is a subpath of $\partial \Gamma_{1},\left|u_{1}\right| \geq 0$ and $\varphi\left(u_{1}\right)$ is a reduced or empty word over $\left\{a_{2}^{ \pm 1}, b_{1}^{ \pm 1}, \ldots, b_{n_{0}-1}^{ \pm 1}\right\}$, while $u_{2}^{-1}$ is a subpath of $\partial \Gamma_{2},\left|u_{2}\right| \geq 0$ and $\varphi\left(u_{2}\right)$ is a reduced or empty word over $\left\{a_{1}^{ \pm 1}\right\}$, see Fig. 9.6. The equality $\left|u_{1}\right|+\left|u_{2}\right|=0$ implies that the faces $\Pi_{1}, \Pi_{2}$ form a reducible pair. This contradiction to $\Delta$ being reduced proves that $\left|u_{1}\right|+\left|u_{2}\right|>0$, whence $\left|\Delta_{1}(2)\right|>0$. 


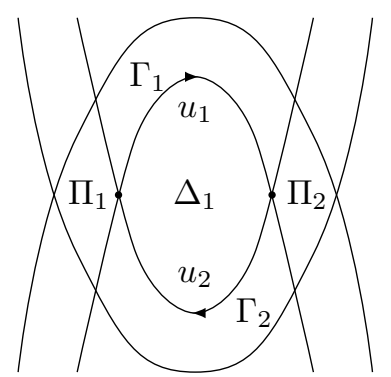

Fig. 9.6

We now pick a disk subdiagram $\Delta_{1}^{\prime}$ in $\Delta$ such that $\left|\Delta_{1}^{\prime}(2)\right|$ is minimal,

$$
\partial \Delta_{1}^{\prime}=u_{1}^{\prime} u_{2}^{\prime}, \quad\left|u_{1}^{\prime}\right|+\left|u_{2}^{\prime}\right|>0
$$

$\varphi\left(u_{1}^{\prime}\right)$ is a reduced or empty word over the alphabet $\left\{a_{2}^{ \pm 1}, b_{1}^{ \pm 1}, \ldots, b_{n_{0}-1}^{ \pm 1}\right\}$, and $\varphi\left(u_{2}\right)$ is a reduced or empty word over $\left\{a_{1}^{ \pm 1}\right\}$. Consider an $a_{1}$-band $\Gamma_{3}$ in $\Delta_{1}^{\prime}$ if $\left|u_{2}^{\prime}\right|>0$ or consider an $a_{2}$-band $\Gamma_{4}$ in $\Delta_{1}^{\prime}$ if $\left|u_{2}^{\prime}\right|=0$ and $\left|u_{1}^{\prime}\right|>0$ such that a connecting line of $\Gamma_{j}, j=3,4$, connects points on $u_{2}^{\prime}$ if $j=3$ or on $u_{1}^{\prime}$ if $j=4$, see Fig. 9.7. It is easy to check that, taking $\Gamma_{3}$, or $\Gamma_{4}$, out of $\Delta_{1}^{\prime}$, we obtain two disk subdiagrams $\Delta_{1,1}^{\prime}, \Delta_{1,2}^{\prime}$ one of which has the above properties of $\Delta_{1}^{\prime}$ and a fewer number of faces, see Fig. 9.7. This contradiction to the minimality of $\Delta_{1}^{\prime}$ completes the proof of Lemma 9.2 .

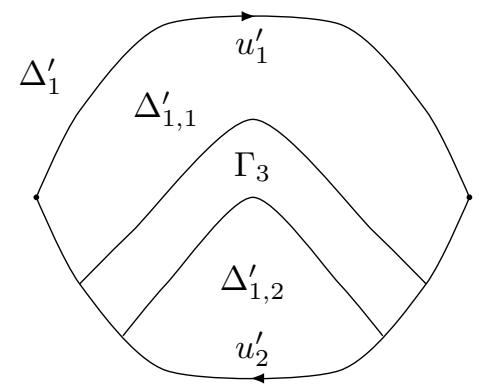

Fig. 9.7

Coming back to the diagrams $\Delta_{0}$ and $\Delta_{0, b}$, see (9.6), we can see from Lemma 9.2 that the number of $a_{1}$-bands in $\Delta_{0, b}$ is at most $\frac{1}{2}\left|\partial \Delta_{0, b}\right|_{a_{1}} \leq \frac{1}{2}\left|\partial \Delta_{0}\right|$. Similarly, the number of $a_{2}$-bands in $\Delta_{0, b}$ is at most $\frac{1}{2}\left|\partial \Delta_{0, b}\right|=\frac{1}{2}\left|\partial \Delta_{0}\right|$. It follows from the definitions and Lemma 9.2 that the number $\left|\Delta_{0, b}(2)\right|$ is equal to the number of intersections of connecting lines of $a_{1}$-bands and $a_{2}$-bands in $\Delta_{0, b}$. Hence, referring to Lemma 9.2 again, we obtain that $\left|\Delta_{0, b}(2)\right| \leq\left(\frac{1}{2}\left|\partial \Delta_{0}\right|\right)^{2}$. In view of (9.6), we finally have

$$
\left|\Delta_{0}(2)\right|=\frac{1}{n_{0}}\left|\Delta_{0, b}(2)\right| \leq \frac{1}{4 n_{0}}\left|\partial \Delta_{0}\right|^{2},
$$

as desired. The proof of Lemma 9.1 is complete.

Suppose $\Delta_{0}$ is a reduced diagram over presentation (1.4), where $\left|n_{1}\right|=\left|n_{2}\right|$. It follows from Lemma 9.2 that $\left|\Delta_{0}(2)\right| \leq \frac{1}{4 n_{0}}\left|\partial \Delta_{0}\right|^{2}$. This inequality means that the space bound (9.1) becomes $O\left((\log |W|)^{3}\right)$ as $n(W) \leq \frac{1}{4 n_{0}}\left|\partial \Delta_{0}\right|^{2}=\frac{1}{4 n_{0}}|W|^{2}$. The space part of Theorem 1.7 is proved. 
To prove the time part of Theorem 1.7, we review the proof of $\mathrm{P}$ part of Theorem 1.4. We observe that our arguments enable us, concurrently with computation of numbers $\lambda(W(i, j)), \mu_{3}(W(i, j))$ for every parameterized subword $W(i, j)$ of $W$ such that $\lambda(W(i, j))=\ell<\infty$, to inductively construct a minimal diagram $\Delta(i, j)$ over (1.4) such that $\varphi\left(\left.\partial\right|_{0} \Delta(i, j)\right) \equiv W(i, j) a_{1}^{-\ell}$. Details of this construction are straightforward in each of the subcases considered in the proof of $\mathrm{P}$ part of Theorem 1.4. Note that this modified algorithm can still be run in time $O\left(|W|^{4}\right)$ for the general case.

If, in addition, $\left|n_{1}\right|=\left|n_{2}\right|$ then the inequality (7.5) can be improved to

$$
|\lambda(W(i, j))| \leq|W(i, j)|, \quad \mu_{3}(W(i, j)) \leq|W(i, j)|^{2} .
$$

Hence, using, as before, binary representation for numbers $\lambda\left(W\left(i^{\prime}, j^{\prime}\right)\right), \mu_{3}\left(W\left(i^{\prime}, j^{\prime}\right)\right)$, we can run inductive computation of numbers $\lambda(U), \mu_{3}(U)$ for $U=W(i, j)$ and construction of a diagram $\Delta(i, j)$ whenever it exists for given $i, j$, in time

$$
O(|W(i, j)| \log |W(i, j)|) .
$$

This improves the bound for running time of our modified algorithm, that computes the numbers $\lambda(W), \mu_{3}(W)$ and constructs a minimal diagram $\Delta(1,|W|)$ for $W$, from $O\left(|W|^{4}\right)$ to $O\left(|W|^{3} \log |W|\right)$, as desired.

Theorem 1.7 is proved.

\section{Polygonal Curves in the Plane and Proofs of Theorems 1.8, 1.9 AND COROLlary 1.10}

Let $\mathcal{T}$ denote a tessellation of the plane $\mathbb{R}^{2}$ into unit squares whose vertices are points with integer coordinates. Let $c$ be a finite closed path in $\mathcal{T}$ so that edges of $c$ are edges of $\mathcal{T}$. Recall that we have the following two types of elementary operations over $c$. If $e$ is an oriented edge of $c, e^{-1}$ is the edge with an opposite to $e$ orientation, and $e e^{-1}$ is a subpath of $c$ so that $c=c_{1} e e^{-1} c_{2}$, where $c_{1}, c_{2}$ are subpaths of $c$, then the operation $c \rightarrow c_{1} c_{2}$ over $c$ is called an elementary homotopy of type 1. Suppose that $c=c_{1} u c_{2}$, where $c_{1}, u, c_{2}$ are subpaths of $c$, and a boundary path $\partial s$ of a unit square $s$ of $\mathcal{T}$ is $\partial s=u v$, where $u, v$ are subpaths of $\partial s$ and either of $u, v$ could be of zero length, i.e., either of $u, v$ could be a single vertex of $\partial s$. Then the operation $c \rightarrow c_{1} v^{-1} c_{2}$ over $c$ is called an elementary homotopy of type 2.

Theorem 1.8. Let $c$ be a finite closed path in a tessellation $\mathcal{T}$ of the plane $\mathbb{R}^{2}$ into unit squares so that edges of $c$ are edges of $\mathcal{T}$. Then a minimal number $m_{2}(c)$ such that there is a finite sequence of elementary homotopies of type 1-2, which turns $c$ into a single point and which contains $m_{2}(c)$ elementary homotopies of type 2, can be computed in deterministic space $O\left((\log |c|)^{3}\right)$ or in deterministic time $O\left(|c|^{3} \log |c|\right)$, where $|c|$ is the length of $c$.

Furthermore, such a sequence of elementary homotopies of type 1-2, which turns $c$ into a single point and which contains $m_{2}(c)$ elementary homotopies of type 2, can also be computed in deterministic space $O\left((\log |c|)^{3}\right)$ or in deterministic time $O\left(|c|^{3} \log |c|\right)$.

Proof. First we assign $\varphi$-labels to edges of the tessellation $\mathcal{T}$. If an edge $e$ goes from a point of $\mathbb{R}^{2}$ with coordinates $(i, j)$ to a point with coordinates $(i+1, j)$, then we set $\varphi(e):=a_{1}$ and $\varphi\left(e^{-1}\right):=a_{1}^{-1}$. If an edge $f$ goes from a point of $\mathbb{R}^{2}$ with coordinates $(i, j)$ to a point with coordinates $(i, j+1)$, then we set $\varphi(f):=a_{2}$ and $\varphi\left(f^{-1}\right):=a_{2}^{-1}$. 
Let $c$ be a finite closed path in $\mathcal{T}$ whose edges are those of $\mathcal{T}$. Without loss of generality, we may assume that $c$ starts at the origin, hence $c_{-}=c_{+}$has coordinates $(0,0)$ (otherwise, we could apply a logspace reduction to achieve this property). Denote $c=\widetilde{f}_{1} \ldots \widetilde{f}_{|c|}$, where $\widetilde{f}_{1}, \ldots, \widetilde{f}_{|c|}$ are edges of $c$, and let

$$
\varphi(c):=\varphi\left(\widetilde{f}_{1}\right) \ldots \varphi\left(\widetilde{f}_{|c|}\right),
$$

where $\varphi(c)$ is a word over the alphabet $\mathcal{A}^{ \pm 1}=\left\{a_{1}^{ \pm 1}, a_{2}^{ \pm 1}\right\}$. Since $c$ is closed, it follows that $\varphi(c) \stackrel{\mathcal{G}_{5}}{=} 1$, where

$$
\mathcal{G}_{5}:=\left\langle a_{1}, a_{2} \| a_{2} a_{1} a_{2}^{-1} a_{1}^{-1}=1\right\rangle .
$$

Lemma 10.1. Suppose that a closed path $c$ in $\mathcal{T}$ can be turned into a point by a finite sequence $\Xi$ of elementary homotopies and $m_{2}(\Xi)$ is the number of elementary homotopies of type 2 in this sequence. Then there is a disk diagram $\Delta$ such that $\varphi(\partial \Delta) \equiv \varphi(c)$ and $|\Delta(2)|=m_{2}(\Xi)$.

Conversely, suppose there is a disk diagram $\Delta$ with $\varphi(\partial \Delta) \equiv \varphi(c)$. Then there is a finite sequence $\Xi$ of elementary homotopies such that $\Xi$ turns $c$ into a point and $m_{2}(\Xi)=|\Delta(2)|$.

Proof. Given a finite sequence $\Xi$ of elementary homotopies $\xi_{1}, \xi_{2}, \ldots$, we can construct a disk diagram $\Delta$ over (10.1) such that $\varphi(\partial \Delta) \equiv \varphi(c)$ and $m_{2}(\Xi)=|\Delta(2)|$. Indeed, starting with a simple closed path $q_{c}$ in the plane $\mathbb{R}^{2}$ (without any tessellation) such that $\varphi\left(q_{c}\right) \equiv \varphi(c)$, we can simulate elementary homotopies in the sequence $\Xi$ so that an elementary homotopy of type 1 is simulated by folding a suitable pair of edges of the path $q_{c}$, see Fig. 10.1(a), and an elementary homotopy of type 2 is simulated by attachment of a face $\Pi$ over (10.1) to the bounded region of $\mathbb{R}^{2}$ whose boundary is $q_{c}$, see Fig. $10.1(\mathrm{~b})$.

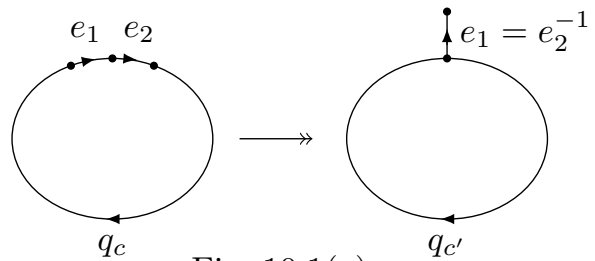

Fig. 10.1(a)

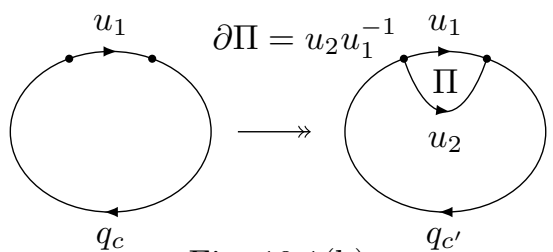

Fig. 10.1(b)

As a result, we will fill out the bounded region of $\mathbb{R}^{2}$ whose boundary path is $q_{c}$ with faces and obtain a required disk diagram $\Delta$ over (10.1).

The converse can be established by a straightforward induction on $|\Delta(2)|$.

It follows from Lemma 10.1 and Theorem 1.7 that a minimal number $m_{2}(c)$ of elementary homotopies of type 2 in a sequence of elementary homotopies that turns the path $c$ into a point can be computed in deterministic space $O\left((\log |\varphi(c)|)^{3}\right)=$ $O\left((\log |c|)^{3}\right)$ or in deterministic time $O\left(|c|^{3} \log |c|\right)$.

It remains to show that a desired sequence of elementary homotopies for $c$ can be computed in deterministic space $O\left((\log |c|)^{3}\right)$ or in deterministic time $O\left(|c|^{3} \log |c|\right)$. We remark that Lemma 10.1 and its proof reveal a close connection between sequences of elementary homotopies that turn $c$ into a point and disk diagrams $\Delta$ over (10.1) such that $\varphi\left(\left.\partial\right|_{0} \Delta\right) \equiv \varphi(c)$. According to Theorem 1.7, we can construct a disk diagram $\Delta_{c}$ such that $\varphi\left(\left.\partial\right|_{0} \Delta_{c}\right) \equiv \varphi(c)$ and $\left|\Delta_{c}(2)\right|=m_{2}(c)$ in space $O\left((\log |c|)^{3}\right)$ 
or in time $O\left(|c|^{3} \log |c|\right)$. Since $\left|\Delta_{c}(2)\right| \leq\left|\partial \Delta_{c}\right|^{2} / 4=|c|^{2} / 4$ by Lemma 9.1 it follows that we can use this diagram $\Delta_{c}$ together with Lemma 10.1 to construct a desired sequence of elementary homotopies in deterministic time $O\left(|c|^{2}\right)$. Therefore, a desired sequence of elementary homotopies can be constructed in deterministic time $O\left(|c|^{3} \log |c|\right)$.

It is also tempting to use this diagram $\Delta_{c}$ together with Lemma 10.1 to construct, in space $O\left((\log |c|)^{3}\right)$, a desired sequence of elementary homotopies which turns $c$ into a point. However, this approach does not quite work for the space part of the proof because inductive arguments of the proof of Lemma 10.1 use intermediate paths whose storage would not be possible in $O\left((\log |c|)^{3}\right)$ space. For this reason, we have to turn back to calculus of pseudobrackets.

Denote $W:=\varphi(c)$. As in Sects. 2, 6, let $P_{W}$ be a path such that $\varphi\left(P_{W}\right) \equiv W$ and vertices of $P_{W}$ are integers $0,1, \ldots,|W|=|c|$. Denote $P_{W}=f_{1} \ldots f_{|c|}$, where $f_{1}, \ldots, f_{|c|}$ are edges of $P_{W}$, and let

$$
\gamma: P_{W} \rightarrow c
$$

be a cellular map such that $\gamma\left(f_{i}\right)=\widetilde{f}_{i}$ for $i=1, \ldots,|W|$. Recall that $c=\widetilde{f}_{1} \ldots \widetilde{f}_{|c|}$. Note that $\gamma(i)=\left(\widetilde{f}_{i}\right)_{+}$, where $i=1, \ldots,|W|$, and $\gamma(0)=\left(\widetilde{f}_{1}\right)_{-}=\left(\widetilde{f}_{|c|}\right)_{+}$.

Suppose $B$ is a pseudobracket system for $W$ and let $B=\left\{b_{1}, \ldots, b_{k}\right\}$, where $b_{i}(2) \leq b_{j}(1)$ if $i<j$. Recall that the $\operatorname{arc} a(b)$ of a pseudobracket $b \in B$ is a subpath of $P_{W}$ such that $a(b)_{-}=b(1)$ and $a(b)_{+}=b(2)$.

We now define a path $\Gamma(B)$ for $B$ in $\mathcal{T}$ so that $\Gamma(B):=\gamma\left(P_{W}\right)=c$ if $k=0$, i.e., $B=\varnothing$, and for $k>0$ we set

$$
\Gamma(B):=c_{0} \delta\left(a\left(b_{1}\right)\right) c_{1} \ldots \delta\left(a\left(b_{k}\right)\right) c_{k},
$$

where $c_{i}=\gamma\left(d_{i}\right)$ and $d_{i}$ is a subpath of $P_{W}$ defined by $\left(d_{i}\right)_{-}=b_{i}(2)$ and $\left(d_{i}\right)_{+}=$ $b_{i+1}(1)$, except for the case when $i=0$, in which case $\left(d_{0}\right)_{-}=0$, and except for the case when $i=k$, in which case $\left(d_{k}\right)_{-}=|W|$. For every $i=1, \ldots, k$, the path $\delta\left(a\left(b_{i}\right)\right)$ in (10.2) is defined by the equalities

$$
\delta\left(a\left(b_{i}\right)\right)_{-}=\gamma\left(b_{i}(1)\right), \quad \delta\left(a\left(b_{i}\right)\right)_{+}=\gamma\left(b_{i}(2)\right)
$$

and by the equality $\varphi\left(\delta\left(a\left(b_{i}\right)\right)\right) \equiv a_{1}^{k_{1}} a_{2}^{k_{2}}$ with some integers $k_{1}, k_{2}$.

Suppose that $B_{0}, B_{1}, \ldots, B_{\ell}$ is an operational sequence of pseudobracket systems for $W$ that corresponds to a sequence $\Omega$ of elementary operations that turns the empty pseudobracket system $B_{0}$ into a final pseudobracket system $B_{\ell}=\left\{b_{\ell, 1}\right\}$. We also assume that $b_{\ell, 1}(4)=\left|\Delta_{c}(2)\right|$ and $\Delta_{c}$ is a minimal disk diagram over (10.1) such that $\varphi\left(\partial \Delta_{c}\right) \equiv W \equiv \varphi(c)$. It follows from Lemmas 9.1, 10.1 that

$$
\left|\Delta_{c}(2)\right|=m_{2}(c) \leq\left|\partial \Delta_{c}\right|^{2} / 4=|W|^{2} / 4 \text {. }
$$

Hence, in view of Lemma 6.13, see also inequalities (7.1)-(7.2), we may assume that, for every pseudobracket $b \in \cup_{i=0}^{\ell} B_{i}$, it is true that

$$
\begin{aligned}
0 & \leq b(1), b(2) \leq|W|, \\
0 & \leq|b(3)| \leq\left(|W|_{a_{1}}+\left(\left|n_{1}\right|+\left|n_{2}\right|\right)\left|\Delta_{c}(2)\right|\right) / 2 \\
& \leq\left(|W|_{a_{1}}+|W|^{2} / 2\right) / 2 \leq|W|^{2} / 2 \\
0 & \leq b(4) \leq\left|\Delta_{c}(2)\right| \leq|W|^{2} / 4
\end{aligned}
$$

Thus the space needed to store a pseudobracket $b \in \cup_{i=0}^{\ell} B_{i}$ is $O(\log |W|)$. 
For every pseudobracket system $B_{i}$, denote $B_{i}=\left\{b_{i, 1}, \ldots, b_{i, k_{i}}\right\}$, where, as above, $b_{i, j}(2) \leq b_{i, j^{\prime}}(1)$ whenever $j<j^{\prime}$.

For every pseudobracket system $B_{i}$, we consider a path $c(i):=\Gamma\left(B_{i}\right)$ in $\mathcal{T}$ defined by the formula (10.2), hence, we have

$$
c(i):=\Gamma\left(B_{i}\right)=c_{0}(i) \delta\left(a\left(b_{i, 1}\right)\right) c_{1}(i) \ldots \delta\left(a\left(b_{i, k_{i}}\right)\right) c_{k_{i}}(i) .
$$

As before, for $B_{0}=\varnothing$, we set $c(0):=\Gamma\left(B_{0}\right)=c$.

Note that the last path $c(\ell)$ in the sequence $c(0), \ldots, c(\ell)$, corresponding to a final pseudobracket system $B_{\ell}=\left\{b_{\ell, 1}\right\}$, where $b_{\ell, 1}=\left(0,|W|, 0, m_{2}(c)\right)$, consists of a single vertex and has the form (10.7) equal to $c(\ell)=c_{0}(\ell) \delta\left(a\left(b_{\ell, 1}\right)\right) c_{1}(\ell)$, where $c_{0}(\ell)=c_{1}(\ell)=c_{-}=c_{+}$and $\delta\left(a\left(b_{\ell, 1}\right)\right)=c$.

Lemma 10.2. Let $b_{i, j} \in B_{i}$ be a pseudobracket. Then $\varphi\left(\delta\left(a\left(b_{i, j}\right)\right)\right) \equiv a_{1}^{b_{i, j}(3)}$, i.e., the points $\gamma\left(b_{i, j}(1)\right), \gamma\left(b_{i, j}(2)\right)$ of $\mathcal{T}$ have the same $y$-coordinate.

Proof. Since the path $\gamma\left(a\left(b_{i, j}\right)\right) \delta\left(a\left(b_{i, j}\right)^{-1}\right.$ in $\mathcal{T}$ is closed, it follows that

$$
\varphi\left(\gamma\left(a\left(b_{i, j}\right)\right)\right) \stackrel{\mathcal{G}_{5}}{=} \varphi\left(\delta\left(a\left(b_{i, j}\right)^{-1}\right),\right.
$$

where $\mathcal{G}_{5}$ is given by presentation (10.1). On the other hand, $\varphi\left(\gamma\left(a\left(b_{i, j}\right)\right) \equiv\right.$ $\varphi\left(a\left(b_{i, j}\right)\right)$. Hence, by Claim (D) of the proof of Lemma 6.12, we get that

$$
\varphi\left(\delta\left(a\left(b_{i, j}\right)\right)\right) \stackrel{\mathcal{G}_{5}}{=} \varphi\left(a\left(b_{i, j}\right) \stackrel{\mathcal{G}_{5}}{=} a_{1}^{b_{i, j}(3)} .\right.
$$

Since $\varphi\left(\delta\left(a\left(b_{i, j}\right)\right)\right) \equiv a_{1}^{k_{1}} a_{2}^{k_{2}}$ with some integers $k_{1}, k_{2}$, it follows that $k_{1}=b_{i, j}(3)$ and $k_{2}=0$, as required.

We now analyze how the path $c(i)$, defined by (10.7), changes in comparison with the path $c(i-1), i \geq 1$.

Suppose that a pseudobracket system $B_{i}, i \geq 1$, is obtained from $B_{i-1}$ by an addition. Then $c(i)=c(i-1)$, hence no change over $c(i-1)$ is done. Note that the form (10.7) does change by an insertion of a subpath consisting of a single vertex which is the $\delta$-image of the arc of the added to $B_{i-1}$ starting pseudobracket.

Assume that a pseudobracket system $B_{i}, i \geq 1$, is obtained from $B_{i-1}$ by an extension of type 1 on the left and $b_{i, j} \in B_{i}$ is obtained from $b_{i-1, j} \in B_{i-1}$ by this elementary operation. Let $a\left(b_{i, j}\right), a\left(b_{i-1, j}\right)$ be the $\operatorname{arcs}$ of $b_{i, j}, b_{i-1, j}$, resp., and let $a\left(b_{i, j}\right)=e_{1} a\left(b_{i-1, j}\right)$, where $e_{1}$ is an edge of $P_{W}$ and $\varphi\left(e_{1}\right)=a_{1}^{\varepsilon_{1}}, \varepsilon_{1}= \pm 1$.

If $\varepsilon_{1} \cdot b_{i-1, j}(3) \geq 0$, then we can see that $c(i)=c(i-1)$ because

$$
c_{j-1}(i-1) \delta\left(a\left(b_{i-1, j}\right)\right)=c_{j-1}(i) \delta\left(a\left(b_{i, j}\right)\right)
$$

and all other syllables of the paths $c(i)$ and $c(i-1)$, as defined in (10.7), are identical.

On the other hand, if $\varepsilon_{1} \cdot b_{i-1, j}(3)<0$, then the subpath $c_{j-1}(i) \delta\left(a\left(b_{i, j}\right)\right)$ of $c(i)$ differs from the subpath $c_{j-1}(i-1) \delta\left(a\left(b_{i-1, j}\right)\right)$ of $c(i-1)$ by cancelation of a subpath $e e^{-1}$, where $e$ is the last edge of $c_{j-1}(i-1), e^{-1}$ is the first edge of $\delta\left(a\left(b_{i-1, j}\right)\right)$, and $\varphi(e)=a_{1}^{-b_{i-1, j}(3) /\left|b_{i-1, j}(3)\right|}$. Since all other syllables of the paths $c(i)$ and $c(i-1)$, as defined in (10.7), are identical, the change of $c(i-1)$, resulting in $c(i)$, can be described as an elementary homotopy of type 1 which deletes a subpath $e e^{-1}$, where $e$ is an edge of $c$ defined by the equalities

$$
e_{+}=\gamma\left(b_{i-1, j}(1)\right), \quad \varphi(e)=a_{1}^{-b_{i-1, j}(3) /\left|b_{i-1, j}(3)\right|} .
$$

The case when a pseudobracket system $B_{i}, i \geq 1$, is obtained from $B_{i-1}$ by an extension of type 1 on the right is similar but we need to make some changes. 
Keeping most of the foregoing notation unchanged, we let $a\left(b_{i, j}\right)=a\left(b_{i-1, j}\right) e_{2}$, where $e_{2}$ is an edge of $P_{W}$ and $\varphi\left(e_{2}\right)=a_{1}^{\varepsilon_{2}}, \varepsilon_{2}= \pm 1$.

If $\varepsilon_{2} \cdot b_{i-1, j}(3) \geq 0$, then as above we conclude that $c(i)=c(i-1)$ because

$$
\delta\left(a\left(b_{i-1, j}\right)\right) c_{j}(i-1)=\delta\left(a\left(b_{i, j}\right)\right) c_{j}(i)
$$

and all other syllables of the paths $c(i)$ and $c(i-1)$, as defined in (10.7), are identical.

If $\varepsilon_{1} \cdot b_{i-1, j}(3)<0$, then we can see from the definitions and from Lemma 10.2 that the path $c(i)$ can be obtained from $c(i-1)$ by an elementary homotopy of type 1 which deletes a subpath $e^{-1} e$, where $e$ is an edge of $c$ defined by the equalities

$$
e_{-}=\gamma\left(b_{i-1, j}(2)\right), \quad \varphi(e)=a_{1}^{-b_{i-1, j}(3) /\left|b_{i-1, j}(3)\right|} .
$$

We conclude that, in the case when $B(i)$ is obtained from $B(i-1)$ by an extension of type 1 , it follows from inequalities (10.4)-(10.6) and from equations (10.8)-(10.9) that the elementary homotopy of type 1 that produces $c(i)$ from $c(i-1)$ can be computed in space $O(\log |W|)$ when the pseudobrackets $b_{i, j} \in B_{i}$ and $b_{i-1, j} \in B_{i-1}$ are known.

Suppose that a pseudobracket system $B_{i}, i \geq 1$, is obtained from $B_{i-1}$ by an extension of type 2 and $b_{i, j} \in B_{i}$ is obtained from $b_{i-1, j} \in B_{i-1}$ by this elementary operation. Denote $a\left(b_{i, j}\right)=e_{1} a\left(b_{i-1, j}\right) e_{2}$, where $e_{1}, e_{2}$ are edges of $P_{W}$ and $\varphi\left(e_{1}\right)=$ $\varphi\left(e_{2}\right)^{-1}=a_{2}^{\varepsilon}, \varepsilon= \pm 1$. According to the definition of an extension of type 2, $b_{i-1, j}(3) \neq 0$ and, in view of the equalities $n_{1}=n_{2}=1$, see (1.4) and (10.1), we can derive from Lemma 10.2 that the path $c(i-1)$ turns into the path $c(i)$ in the following fashion. Denote $c_{j-1}(i-1)=\widetilde{c}_{j-1}(i-1) \widetilde{e}_{1}$ and $c_{j}(i-1)=\widetilde{e}_{2} \widetilde{c}_{j}(i-1)$, where $\widetilde{c}_{j-1}(i-1), \widetilde{c}_{j}(i-1)$ are subpaths of $c_{j-1}(i-1), c_{j}(i-1)$, resp., and $\widetilde{e}_{1}, \widetilde{e}_{2}$ are edges such that $\gamma\left(e_{i^{\prime}}\right)=\widetilde{e}_{i^{\prime}}, i^{\prime}=1,2$. Then $c_{j-1}(i)=\widetilde{c}_{j-1}(i-1), c_{j}(i)=\widetilde{c}_{j}(i-1)$, and the path $\delta\left(a\left(b_{i, j}\right)\right)$ has the properties that

$$
\begin{aligned}
& \delta\left(a\left(b_{i, j}\right)\right)_{-}=\left(\widetilde{e}_{1}\right)_{-}, \quad \delta\left(a\left(b_{i, j}\right)\right)_{+}=\left(\widetilde{e}_{2}\right)_{+}, \\
& \varphi\left(\delta\left(a\left(b_{i-1, j}\right)\right)\right) \equiv a_{1}^{b_{i-1, j}(3)} \equiv \varphi\left(\delta\left(a\left(b_{i, j}\right)\right)\right),
\end{aligned}
$$

see Fig. 10.2, and the other syllables of the paths $c(i)$ and $c(i-1)$, as defined in (10.7), are identical.

$$
\begin{aligned}
& c_{j-1}(i-1)=\widetilde{c}_{j-1}(i-1) \widetilde{e}_{1} \quad \delta\left(a\left(b_{i-1, j}\right)\right) \quad c_{j}(i-1)=\widetilde{e}_{2} \widetilde{c}_{j}(i-1)
\end{aligned}
$$

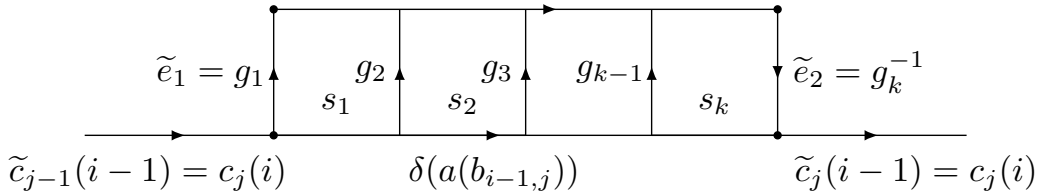

Fig. 10.2

Denote $k:=\left|b_{i-1, j}(3)\right|=\left|b_{i, j}(3)\right|>0$ and let

$$
\delta\left(a\left(b_{i-1, j}\right)\right)=d_{i-1,1} \ldots d_{i-1, k}, \quad \delta\left(a\left(b_{i, j}\right)\right)=d_{i, 1} \ldots d_{i, k},
$$

where $d_{i^{\prime}, j^{\prime}}$ are edges of the paths $\delta\left(a\left(b_{i-1, j}\right)\right), \delta\left(a\left(b_{i, j}\right)\right)$. Also, we let $g_{1}, \ldots, g_{k}$ be the edges of $\mathcal{T}$ such that $\varphi\left(g_{i^{\prime}}\right)=\varphi\left(\widetilde{e}_{1}\right)=a_{2}^{\varepsilon}$ and $\left(g_{i^{\prime}}\right)_{-}=\left(d_{i, i^{\prime}}\right)_{-}$for $i^{\prime}=$ $1, \ldots, k$, in particular, $g_{1}=\widetilde{e}_{1}$ and $g_{k}=\widetilde{e}_{2}^{-1}$, see Fig. 10.2. Then there are $k$ elementary homotopies of type 2 which turn $c(i-1)$ into $c(i)$ and which use $k$ 
squares of the region bounded by the closed path $\widetilde{e}_{1} \delta\left(a\left(b_{i-1, j}\right)\right) \widetilde{e}_{2} \delta\left(a\left(b_{i, j}\right)\right)^{-1}$, see Fig. 10.2. For example, the first elementary homotopy of type 2 replaces the subpath $\widetilde{e}_{1} d_{i-1,1}=g_{1} d_{i-1,1}$ by $d_{i, 1} g_{2}$. Note that $\partial s_{1}=g_{1} d_{i-1,1}\left(d_{i, 1} g_{2}\right)^{-1}$ is a (negatively orientated) boundary path of a square $s_{1}$ of $\mathcal{T}$. The second elementary homotopy of type 2 (when $k \geq 2$ ) replaces the subpath $g_{2} d_{i-1,2}$ by $d_{i, 2} g_{3}$, where $\partial s_{2}=$ $g_{2} d_{i-1,2}\left(d_{i, 2} g_{3}\right)^{-1}$ is a (negatively orientated) boundary path of a square $s_{2}$ of $\mathcal{T}$, and so on.

In view of inequalities (10.4)-(10.6), these $k=\left|b_{i-1, j}(3)\right|$ elementary homotopies of type 2 that produce $c(i)$ from $c(i-1)$ can be computed in space $O(\log |W|)$ when the pseudobrackets $b_{i, j} \in B_{i}$ and $b_{i-1, j} \in B_{i-1}$ are available.

Suppose that a pseudobracket system $B_{i}, i \geq 1$, is obtained from $B_{i-1}$ by an extension of type 3 and $b_{i, j} \in B_{i}$ is obtained from $b_{i-1, j} \in B_{i-1}$ by this elementary operation. By the definition of an extension of type $3, b_{i-1, j}(3)=0$ and $a\left(b_{i, j}\right)=$ $e_{1} a\left(b_{i-1, j}\right) e_{2}$, where $e_{1}, e_{2}$ are edges of $P_{W}$ and $\varphi\left(e_{1}\right)=\varphi\left(e_{2}\right)^{-1} \neq a_{1}^{ \pm 1}$. Hence, $\varphi\left(e_{1}\right)=\varphi\left(e_{2}\right)^{-1}=a_{2}^{\varepsilon}, \varepsilon= \pm 1$. It follows from Lemma 10.2 that

$$
\delta\left(a\left(b_{i-1, j}\right)\right)=c_{j-1}(i-1)_{+}=c_{j}(i-1)_{-}, \quad \delta\left(a\left(b_{i, j}\right)\right)=c_{j-1}(i)_{+}=c_{j}(i)_{-} .
$$

As above, denote $c_{j-1}(i-1)=\widetilde{c}_{j-1}(i-1) \widetilde{e}_{1}$ and $c_{j}(i-1)=\widetilde{e}_{2} \widetilde{c}_{j}(i-1)$, where $\widetilde{c}_{j-1}(i-1), \widetilde{c}_{j}(i-1)$ are subpaths of $c_{j-1}(i-1), c_{j}(i-1)$, resp., and $\widetilde{e}_{1}, \widetilde{e}_{2}$ are edges such that $\gamma\left(e_{i^{\prime}}\right)=\widetilde{e}_{i^{\prime}}, i^{\prime}=1,2$. Then it follows from the definitions that $c_{j-1}(i)=\widetilde{c}_{j-1}(i-1), c_{j}(i)=\widetilde{c}_{j}(i-1)$ and that all other syllables of the paths $c(i)$ and $c(i-1)$, as defined in (10.7), are identical. Hence, the change of the path $c(i-1)$ into $c(i)$ can be described as an elementary homotopy of type 1 that deletes the subpath $\widetilde{e}_{1} \widetilde{e}_{2}$ of $c(i-1)$. Since $\left(e_{1}\right)_{+}=b_{i-1, j}(1)$ and $\widetilde{e}_{1}=\gamma\left(e_{1}\right)$, it is easy to see from inequalities (10.4)-(10.6) that we can compute this elementary homotopy of type 1 in space $O(\log |W|)$ when the pseudobrackets $b_{i, j} \in B_{i}$ and $b_{i-1, j} \in B_{i-1}$ are given.

Suppose that a pseudobracket system $B_{i}, i \geq 1$, is obtained from $B_{i-1}$ by a merger operation and $b_{i, j} \in B_{i}$ is obtained from pseudobrackets $b_{i-1, j}, b_{i-1, j+1} \in$ $B_{i-1}$ by this merger.

First assume that $b_{i-1, j}(3) \cdot b_{i-1, j+1}(3) \geq 0$. It follows from Lemma 10.2 and from the definitions that

$$
c_{j}(i-1)=\delta\left(a\left(b_{i-1, j}\right)\right)_{+}, \quad \delta\left(a\left(b_{i-1, j}\right)\right) c_{j}(i-1) \delta\left(a\left(b_{i-1, j+1}\right)\right)=\delta\left(a\left(b_{i, j}\right)\right)
$$

and all other syllables of the paths $c(i)$ and $c(i-1)$, as defined in (10.7), are identical. Therefore, we have the equality of paths $c(i-1)=c(i)$ in this case. Note that factorizations (10.7) of $c(i)$ and $c(i-1)$ are different.

Now assume that $b_{i-1, j}(3) \cdot b_{i-1, j+1}(3)<0$. It follows from Lemma 10.2 and from the definitions that $c_{j}(i-1)=\delta\left(a\left(b_{i-1, j}\right)\right)_{+}$and that the subpath $\delta\left(a\left(b_{i, j}\right)\right)$ of $c(i)$ can be obtained from the subpath $\delta\left(a\left(b_{i-1, j}\right)\right) c_{j}(i-1) \delta\left(a\left(b_{i-1, j+1}\right)\right)$ of $c(i-1)$ by cancelation of $\min \left(\left|b_{i-1, j}(3)\right|,\left|b_{i-1, j+1}(3)\right|\right)$ pairs of edges in $c(i-1)$ so that the last edge of $\delta\left(a\left(b_{i-1, j}\right)\right)$ is canceled with the first edge of $\delta\left(a\left(b_{i-1, j+1}\right)\right)$ as a subpath $e e^{-1}$ in the definition of an elementary homotopy of type 1 and so on until a shortest path among $\delta\left(a\left(b_{i-1, j}\right)\right), \delta\left(a\left(b_{i-1, j+1}\right)\right)$ completely cancels. Note that all other syllables of the paths $c(i)$ and $c(i-1)$, as defined in (10.7), are identical. Thus the path $c(i)$ can be obtained from $c(i-1)$ by $\min \left(\left|b_{i-1, j}(3)\right|,\left|b_{i-1, j+1}(3)\right|\right)$ elementary homotopies of type 1 which, in view of (10.4)-(10.6), can be computed 
in space $O(\log |W|)$ when the pseudobrackets $b_{i, j} \in B_{i}$ and $b_{i-1, j}, b_{i-1, j+1} \in B_{i-1}$ are known.

Recall that, in the proof of Theorem 1.7 we devised an algorithm $\mathfrak{A}_{n}$ that, in deterministic space (9.1), constructs a sequence of elementary operations $\Omega$ and a corresponding sequence of pseudobracket systems $B_{0}, \ldots, B_{\ell}$ for $W$ such that $B_{0}$ is empty, $B_{\ell}=\left\{b_{\ell, 1}\right\}$ is final and $b_{\ell, 1}(4)=|\Delta(2)| \leq n$, where $\Delta$ is a minimal disk diagram over (10.1) with $\varphi(\partial \Delta) \equiv W$. In view of inequalities (10.3), we may assume that $n=|W|^{2} / 4$, hence, the bound (9.1) becomes $O\left((\log |W|)^{3}\right)$ and, as we saw in (10.4)-(10.6), every pseudobracket $b \in \cup_{i=0}^{\ell} B_{i}$ requires space $O(\log |W|)$ to store. As was discussed above, when given pseudobracket systems $B_{i-1}$ and $B_{i}$, we can construct, in space $O(\log |W|)$, a sequence of elementary homotopies that turns the path $c(i-1)$ into $c(i)$. Since the sequence of pseudobracket systems $B_{0}, \ldots, B_{\ell}$ for $W \equiv \varphi(c)$ is constructible in space $O\left((\log |W|)^{3}\right)$, it follows that a sequence $\Xi$ of elementary homotopies of type 1-2 that turns the path $c=c(0)$ into a vertex $c(\ell)=c_{-}=c_{+}$can also be constructed in deterministic space $O\left((\log |W|)^{3}\right)$. This completes the proof of Theorem 1.8 .

Recall that a polygonal closed curve $c$ in the plane $\mathbb{R}^{2}$, equipped with a tessellation $\mathcal{T}$ into unit squares, consists of finitely many line segments $c_{1}, \ldots, c_{k}, k>0$, whose endpoints are vertices of $\mathcal{T}, c=c_{1} \ldots c_{k}$, and $c$ is closed, i.e., $c_{-}=c_{+}$. If $c_{i} \subset \mathcal{T}$ then the $\mathcal{T}$-length $\left|c_{i}\right|_{\mathcal{T}}$ of $c_{i}$ is the number of edges of $c_{i}$. If $c_{i} \not \subset \mathcal{T}$ then the $\mathcal{T}$-length $\left|c_{i}\right|_{\mathcal{T}}$ of $c_{i}$ is the number of connected components in $c_{i} \backslash \mathcal{T}$. We assume that $\left|c_{i}\right|_{\mathcal{T}}>0$ for every $i$ and set $|c|_{\mathcal{T}}:=\sum_{i=1}^{k}\left|c_{i}\right|_{\mathcal{T}}$.

Theorem 1.9. Suppose that $n \geq 1$ is a fixed integer and $c$ is a polygonal closed curve in the plane $\mathbb{R}^{2}$ with given tessellation $\mathcal{T}$ into unit squares. Then, in deterministic space $O\left(\left(\log |c|_{\mathcal{T}}\right)^{3}\right)$ or in deterministic time $O\left(|c|_{\mathcal{T}}^{n+3} \log |c|_{\mathcal{T}}\right)$, one can compute a rational number $r_{n}$ such that $\left|A(c)-r_{n}\right|<\frac{1}{|c|_{J}^{n}}$.

In particular, if the area $A(c)$ defined by $c$ is known to be an integer multiple of $\frac{1}{L}$, where $L>0$ is a given integer and $L<|c|_{\mathcal{T}}^{n} / 2$, then $A(c)$ can be computed in deterministic space $O\left(\left(\log |c|_{\mathcal{T}}\right)^{3}\right)$ or in deterministic time $O\left(|c|_{\mathcal{T}}^{n+3} \log |c|_{\mathcal{T}}\right)$.

Proof. As above, let $c=c_{1} \ldots c_{k}$, where each $c_{i}$ is a line segment of $c$ of positive length that connects vertices of $\mathcal{T}$. For every $c_{i}$, we define an approximating path $\zeta\left(c_{i}\right)$ such that $\zeta\left(c_{i}\right)_{-}=\left(c_{i}\right)_{-}, \zeta\left(c_{i}\right)_{+}=\left(c_{i}\right)_{+}$and $\zeta\left(c_{i}\right) \subset \mathcal{T}$. If $c_{i} \subset \mathcal{T}$ then we set $\zeta\left(c_{i}\right):=c_{i}$.

Assume that $c_{i} \not \subset \mathcal{T}$. let $R_{i}$ be a rectangle consisting of unit squares of $\mathcal{T}$ so that $c_{i}$ is a diagonal of $R_{i}$. Consider the set $N\left(c_{i}\right) \subseteq R_{i}$ of all squares $s$ of $R_{i}$ such that the intersection $s \cap c_{i}$ is not empty and is not a single point. Assuming that the boundary path $\partial N\left(c_{i}\right)$ is negatively, i.e., clockwise, oriented, we represent $\partial N\left(c_{i}\right)$ in the form

$$
\partial N\left(c_{i}\right)=q_{1}\left(c_{i}\right) q_{2}\left(c_{i}\right)^{-1},
$$

where $q_{1}\left(c_{i}\right), q_{2}\left(c_{i}\right)^{-1}$ are subpath of $\partial N\left(c_{i}\right)$ defined by the equalities

$$
q_{1}\left(c_{i}\right)_{-}=q_{2}\left(c_{i}\right)_{-}=\left(c_{i}\right)_{-}, \quad q_{1}\left(c_{i}\right)_{+}=q_{2}\left(c_{i}\right)_{+}=\left(c_{i}\right)_{+} .
$$

It is easy to see that these equations uniquely determine the paths $q_{1}\left(c_{i}\right), q_{2}\left(c_{i}\right)$, see Fig. 10.3. 


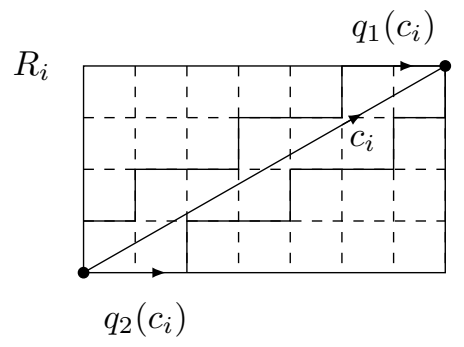

Fig. 10.3

Lemma 10.3. Suppose $c_{i}, R_{i}$ are defined as above, $c_{i} \not \subset \mathcal{T}$, and $\left|R_{i}\right|_{x},\left|R_{i}\right|_{y}$ are the lengths of horizontal, vertical, resp., sides of the rectangle $R_{i}$. Then

$$
\max \left(\left|R_{i}\right|_{x},\left|R_{i}\right|_{y}\right) \leq\left|c_{i}\right|_{\mathcal{T}} \leq 2 \max \left(\left|R_{i}\right|_{x},\left|R_{i}\right|_{y}\right),
$$

and the area $A\left(c_{i} q_{1}\left(c_{i}\right)^{-1}\right)$ bounded by the polygonal closed curve $c_{i} q_{1}\left(c_{i}\right)^{-1}$ satisfies

$$
A\left(c_{i} q_{1}\left(c_{i}\right)^{-1}\right) \leq\left|c_{i}\right|_{\mathcal{T}}
$$

In addition, the paths $q_{1}\left(c_{i}\right), q_{2}\left(c_{i}\right)$ can be constructed in deterministic space $O\left(\log |c|_{\mathcal{T}}\right)$ or in deterministic time $O\left(\left|c_{i}\right|_{\mathcal{T}} \log |c|_{\mathcal{T}}\right)$.

Proof. We say that all unit squares of $R_{i}$ whose points have $x$-coordinates in the same range compose a column of $R_{i}$. Similarly, we say that all unit squares of $R_{i}$ whose points have $y$-coordinates in the same range compose a row of $R_{i}$. Since $c_{i}$ is a diagonal of $R_{i}$, it follows that $c_{i}$ has an intersection, different from a single point, with a unit square from every row and from every column of $R_{i}$. Hence, in view of the definition of the $\mathcal{T}$-length $\left|c_{i}\right|_{\mathcal{T}}$ of $c_{i}$, we have $\max \left(\left|R_{i}\right|_{x},\left|R_{i}\right|_{y}\right) \leq\left|c_{i}\right|_{\mathcal{T}}$.

It follows from the definitions of the region $N\left(R_{i}\right) \subseteq R_{i}$ and the $\mathcal{T}$-length $\left|c_{i}\right|_{\mathcal{T}}$ that the number of unit squares in $N\left(R_{i}\right)$ is $\left|c_{i}\right|_{\mathcal{T}}$, hence, the area $A\left(N\left(R_{i}\right)\right)$ is equal to $\left|c_{i}\right|_{\mathcal{T}}$. Since the closed curve $c_{i} q_{1}\left(c_{i}\right)^{-1}$ is contained in $N\left(R_{i}\right)$ and it can be turned into a simple curve by an arbitrarily small deformation, it follows that $A\left(c_{i} q_{1}\left(c_{i}\right)^{-1}\right) \leq A\left(N\left(R_{i}\right)\right)=\left|c_{i}\right|_{\mathcal{T}}$, as required in (10.11).

Let $\mathrm{sl}\left(c_{i}\right)$ denote the slope of the line that goes through $c_{i}$. If $\left|\mathrm{sl}\left(c_{i}\right)\right| \leq 1$ then we can see that $N\left(c_{i}\right)$ contains at most 2 squares in every column of $R_{i}$. On the other hand, if $\left|\mathrm{sl}\left(c_{i}\right)\right| \geq 1$ then $N\left(c_{i}\right)$ contains at most 2 squares in every row of $R_{i}$. Therefore, $A\left(N\left(c_{i}\right)\right) \leq 2 \max \left(\left|R_{i}\right|_{x},\left|R_{i}\right|_{y}\right)$. Since $A\left(N\left(c_{i}\right)\right)=\left|c_{i}\right|_{\mathcal{T}}$, the inequalities (10.10) are proven.

Note that, moving along columns of $R_{i}$ if sl $\left(c_{i}\right) \leq 1$ or moving along rows of $R_{i}$ if $\mathrm{sl}\left(c_{i}\right)>1$, we can detect all squares of $R_{i}$ that belong to the region $N\left(c_{i}\right)$ in space $O\left(\log |c|_{\mathcal{T}}\right)$ or in time $O\left(\left|c_{i}\right|_{\mathcal{T}} \log |c|_{\mathcal{T}}\right)$ by checking which squares in current column or row are intersected by $c_{i}$ in more than one point. This implies that the paths $q_{1}\left(c_{i}\right), q_{2}\left(c_{i}\right)$ can also be constructed in space $O\left(\log |c|_{\mathcal{T}}\right)$ or in time $O\left(\left|c_{i}\right|_{\mathcal{T}} \log |c|_{\mathcal{T}}\right)$, as desired.

For $c_{i} \not \subset \mathcal{T}$, define $\zeta\left(c_{i}\right):=q_{1}\left(c_{i}\right)$. Recall that $\zeta\left(c_{i}\right)=c_{i}$ if $c_{i} \subset \mathcal{T}$. We can now define an approximating closed path $\zeta(c)$ in $\mathcal{T}$ for $c$ by setting

$$
\zeta(c):=\zeta\left(c_{1}\right) \ldots \zeta\left(c_{k}\right) .
$$


By Lemma 10.3 ,

$$
|A(c)-A(\zeta(c))| \leq \sum_{i=1}^{k} A\left(c_{i} \zeta\left(c_{i}\right)^{-1}\right) \leq \sum_{i=1}^{k}\left|c_{i}\right|_{\mathcal{T}}=|c|_{\mathcal{T}} .
$$

Consider a refined tessellation $\mathcal{T}_{M}$, where $M>1$ is an integer, so that every unit square $s$ of $\mathcal{T}$ is divided into $M^{2}$ congruent squares each of area $M^{-2}$. We repeat the foregoing definitions of the lengths $\left|c_{i}\right|_{\mathcal{T}_{M}},|c|_{\mathcal{T}_{M}}$, the paths $q_{j, M}\left(c_{i}\right), j=1,2$, $i=1, \ldots, k$, rectangles $R_{i, M}$, regions $N_{M}\left(c_{i}\right) \subseteq R_{i, M}$, and approximating paths $\zeta_{M}\left(c_{i}\right), \zeta_{M}(c)$ with respect to the refined tessellation $\mathcal{T}_{M}$ in place of $\mathcal{T}$.

Lemma 10.4. Suppose $c_{i} \not \subset \mathcal{T}$, and $\left|R_{i, M}\right|_{x},\left|R_{i, M}\right|_{y}$ denote the path length of horizontal, vertical, resp., sides of the rectangle $R_{i, M}$. Then $\left|R_{i, M}\right|_{x}=M\left|R_{i}\right|_{x}$, $\left|R_{i, M}\right|_{y}=M\left|R_{i}\right|_{y}$,

$$
\max \left(\left|R_{i, M}\right|_{x},\left|R_{i, M}\right|_{y}\right) \leq\left|c_{i}\right|_{\mathcal{T}_{M}} \leq 2 \max \left(\left|R_{i, M}\right|_{x},\left|R_{i, M}\right|_{y}\right),
$$

and the area bounded by the polygonal closed curve $c_{i} q_{1, M}\left(c_{i}\right)^{-1}$ satisfies

$$
A\left(c_{i} q_{1, M}\left(c_{i}\right)^{-1}\right) \leq M^{-2}\left|c_{i}\right|_{\mathcal{T}_{M}} .
$$

In addition, we have

$$
M\left|c_{i}\right|_{\mathcal{T}} / 2 \leq\left|c_{i}\right|_{\mathcal{T}_{M}} \leq 2 M\left|c_{i}\right|_{\mathcal{T}}
$$

and the paths $q_{1, M}\left(c_{i}\right), q_{2, M}\left(c_{i}\right)$ can be constructed in deterministic space

$$
O\left(\log |c|_{\mathcal{T}_{M}}\right)=O\left(\log \left(M|c|_{\mathcal{T}}\right)\right)
$$

or in deterministic time $O\left(\left|c_{i}\right|_{\mathcal{T}_{M}} \log |c|_{\mathcal{T}_{M}}\right)=O\left(M\left|c_{i}\right|_{\mathcal{T}} \log \left(M|c|_{\mathcal{T}}\right)\right)$.

Proof. The equalities $\left|R_{i, M}\right|_{x}=M\left|R_{i}\right|_{x},\left|R_{i, M}\right|_{y}=M\left|R_{i}\right|_{y}$ are obvious from the definitions. Proofs of inequalities (10.12)-(10.13) are analogous to the proofs of inequalities (10.10) -10.11) of Lemma 10.3 with the correction that the area of a square of the tessellation $\mathcal{T}_{M}$ is now $M^{-2}$.

The inequalities (10.14) follow from inequalities (10.10), (10.12) and imply that the space and time bounds $O\left(\log |c|_{\mathcal{T}_{M}}\right), O\left(\left|c_{i}\right|_{\mathcal{T}_{M}} \log |c|_{\mathcal{T}_{M}}\right)$, that are obtained as corresponding bounds of Lemma 10.3, can be rewritten in the form $O\left(\log \left(M|c|_{\mathcal{T}}\right)\right)$, $O\left(M\left|c_{i}\right|_{\mathcal{T}} \log \left(M|c|_{\mathcal{T}}\right)\right)$, resp.

It follows from the definitions and Lemma 10.4 that

$$
\begin{aligned}
\left|A(c)-A\left(\zeta_{M}(c)\right)\right| & \leq \sum_{i=1}^{k} A\left(c_{i} \zeta_{M}\left(c_{i}\right)^{-1}\right) \leq M^{-2} \sum_{i=1}^{k}\left|c_{i}\right|_{\mathcal{T}_{M}} \\
& \leq M^{-2} \sum_{i=1}^{k} 2 M\left|c_{i}\right|_{\mathcal{T}}=2 M^{-1}|c|_{\mathcal{T}}
\end{aligned}
$$

and that the path $\zeta_{M}\left(c_{i}\right) \subset \mathcal{T}_{M}$ can be computed in space $O\left(\log \left(M|c|_{\mathcal{T}}\right)\right)$ or in time $O\left(M\left|c_{i}\right|_{\mathcal{T}} \log \left(M|c|_{\mathcal{T}}\right)\right)$.

Setting $M:=|c|_{\mathcal{T}}^{n+2}$, where $n \geq 1$ is a fixed integer, we can see from Lemma 10.4 that the closed path $\zeta_{M}(c) \subset \mathcal{T}_{M}$ can be constructed in space $O\left(\log |c|_{\mathcal{T}}\right)$ or in time $O\left(|c|_{\mathcal{T}}^{n+3} \log |c|_{\mathcal{T}}\right)$. Hence, by Theorem 1.8 and Lemma 10.4, the area $A\left(\zeta_{M}(c)\right)$ can be computed in space $O\left(\left(\log |c|_{\mathcal{T}_{M}}\right)^{3}\right)=O\left(\left(\log |c|_{\mathcal{T}}\right)^{3}\right)$ or in time 
$O\left(|c|_{\mathcal{T}_{M}}^{3} \log |c|_{\mathcal{T}_{M}}\right)=O\left(|c|_{\mathcal{T}^{n}}^{n+3} \log |c|_{\mathcal{T}}\right)$. The inequality (10.15) together with $M=$ $|c|_{\mathcal{T}}^{n+2}$ imply that

$$
\left|A(c)-A\left(\zeta_{M}(c)\right)\right| \leq 2 M^{-1}|c|_{\mathcal{T}}<|c|_{\mathcal{T}}^{-n},
$$

here we may assume $|c|_{\mathcal{T}}>2$ for otherwise $A(c)=0$ and $r_{n}=0$.

Finally, suppose that the area $A(c)$ is known to be an integer multiple of $\frac{1}{L}$, where $L>0$ is an integer with $L<|c|_{\mathcal{T}}^{n} / 2$. Applying the foregoing approximation result, in deterministic space $O\left(\left(\log |c|_{\mathcal{T}}\right)^{3}\right)$ or in deterministic time $O\left(|c|_{\mathcal{T}}^{n+3} \log |c|_{\mathcal{T}}\right)$, we can compute a rational number $r_{n}=A\left(\zeta_{M}(c)\right)$, where $M=|c|_{\mathcal{T}}^{n+2}$, such that the inequality (10.16) holds true. It follows from Lemma 9.1, inequalities (10.14) and the definitions that both numerator and denominator of $r_{n}$ are nonnegative integers that do not exceed

$$
\max \left(|c|_{\mathcal{T}_{M}}^{2} / 4, M^{2}\right) \leq \max \left(M^{2}|c|_{\mathcal{T}}^{2}, M^{2}\right) \leq|c|_{\mathcal{T}}^{2(n+3)}
$$

Hence, a binary representation of $r_{n}$ takes space $O\left(\log |c|_{\mathcal{T}}\right)$.

Since it is known that $\frac{1}{L}>\frac{2}{|c|_{\mathcal{T}}^{n}}$, it follows from (10.16) that there is at most one integer multiple of $\frac{1}{L}$ at the distance $<\frac{1}{|c|_{\mathcal{T}}^{n}}$ from $r_{n}$. This means that an integer multiple of $\frac{1}{L}$ closest to $r_{n}$ is the area $A(c)$.

Since $r_{n}$ and $L$ are available and their binary representations take space $O\left(\log |c|_{\mathcal{T}}\right)$, it follows that a closest to $r_{n}$ integer multiple of $\frac{1}{L}$, can be computed in space $O\left(\log |c|_{\mathcal{T}}\right)$ or in time $O\left(\left(\log |c|_{\mathcal{T}}\right)^{2}\right)$ and this will be the desired area $A(c)$.

Thus the area $A(c)$ bounded by $c$ can be computed in space $O\left(\log |c|_{\mathcal{T}}^{3}\right)$ or in time $O\left(|c|_{\mathcal{T}}^{n+3} \log |c|_{\mathcal{T}}\right)$. Theorem 1.9 is proved.

Corollary 1.10. Let $K \geq 1$ be a fixed integer and let $c$ be a polygonal closed curve in the plane $\mathbb{R}^{2}$ with given tessellation $\mathcal{T}$ into unit squares such that $c$ has one of the following two properties (a)-(b).

(a) If $c_{i}, c_{j}$ are two nonparallel line segments of $c$ then their intersection point, if it exists, has coordinates that are integer multiples of $\frac{1}{K}$.

(b) If $c_{i}$ is a line segment of $c$ and $a_{i, x}, a_{i, y}$ are coprime integers such that the line given by an equation $a_{i, x} x+a_{i, y} y=b_{i}$, where $b_{i}$ is an integer, contains $c_{i}$, then $\max \left(\left|a_{i, x}\right|,\left|a_{i, y}\right|\right) \leq K$.

Then the area $A(c)$ defined by c can be computed in deterministic space $O\left(\left(\log |c|_{\mathcal{T}}\right)^{3}\right)$ or in deterministic time $O\left(|c|_{\mathcal{T}}^{n+3} \log |c|_{\mathcal{T}}\right)$, where $n$ depends on $K$.

In particular, if $\mathcal{T}_{*}$ is a tessellation of the plane $\mathbb{R}^{2}$ into equilateral triangles of unit area, or into regular hexagons of unit area, and $q$ is a finite closed path in $\mathcal{T}_{*}$ whose edges are edges of $\mathcal{T}_{*}$, then the area $A(q)$ defined by $q$ can be computed in deterministic space $O\left((\log |q|)^{3}\right)$ or in deterministic time $O\left(|q|^{5} \log |q|\right)$.

Proof. Assume that the property (a) holds for $c$. Let $t$ be a triangle in the plane whose vertices $v_{1}, v_{2}, v_{3}$ are points of the intersection of some nonparallel segments of $c$. Then, using the determinant formula

$$
A(t)=\frac{1}{2}\left|\operatorname{det}\left[\overrightarrow{v_{1} v_{2}}, \overrightarrow{v_{1} v_{3}}\right]\right|
$$

for the area $A(t)$ of $t$, we can see that $A(t)$ is an integer multiple of $\frac{1}{2 K^{2}}$. Since $A(c)$ is the sum of areas of triangles such as $t$ discussed above, it follows that $A(c)$ is also an integer multiple of $\frac{1}{2 K^{2}}$. Taking $n$ so that

$$
|c|_{\mathcal{T}}^{n}>4 K^{2}
$$


we see that Theorem 1.9 applies with $L=2 K^{2}$ and yields the desired conclusion.

Suppose that the property (b) holds for $c$. By the Cramer's rule applied to the system of two linear equations

$$
\begin{aligned}
& a_{i, x} x+a_{i, y} y=b_{i} \\
& a_{j, x} x+a_{j, y} y=b_{j}
\end{aligned}
$$

which, as in property (b), define the lines that contain nonparallel segments $c_{i}, c_{j}$, we have that the intersection point of $c_{i}$ and $c_{j}$ has rational coordinates whose denominators do not exceed

$$
\left|\operatorname{det}\left[\begin{array}{ll}
a_{i, x} & a_{i, y} \\
a_{j, x} & a_{j, y}
\end{array}\right]\right| \leq 2 K^{2} .
$$

This means that coordinates of the intersection point of two nonparallel line segments $c_{i}, c_{j}$ of $c$ are integer multiples of $\frac{1}{\left(2 K^{2}\right) !}$ and the case when the property (b) holds for $c$ is reduced to the case when the property (a) holds for $c$ with $K^{\prime}=\left(2 K^{2}\right)$ !.

It remains to show the last claim of Corollary 1.10, The case when $q$ is a closed path in the tessellation $\mathcal{T}_{6}$ of the plane $\mathbb{R}^{2}$ into regular hexagons of unit area can be obviously reduced to the case when $q$ is a closed path in the tessellation $\mathcal{T}_{3}$ of the plane $\mathbb{R}^{2}$ into equilateral triangles of unit area. For this reason we discuss the latter case only.

Consider the standard tessellation $\mathcal{T}=\mathcal{T}_{4}$ of the plane $\mathbb{R}^{2}$ into unit squares and draw a diagonal in each square $s$ of $\mathcal{T}_{4}$ which connects the lower left vertex and the upper right vertex of $s$. Let $\mathcal{T}_{4,1}$ denote thus obtained tessellation of $\mathbb{R}^{2}$ into triangles of area $1 / 2$. Note that $\mathcal{T}_{3}$ and $\mathcal{T}_{4,1}$ are isomorphic as graphs and the areas of corresponding triangles differ by a coefficient of $2^{ \pm 1}$. This isomorphism enables us to define the image $q^{\prime}$ in $\mathcal{T}_{4,1}$ of a closed path $q$ in $\mathcal{T}_{3}$. It is clear that the area $A\left(q^{\prime}\right)$ defined by $q^{\prime}$ relative to $\mathcal{T}_{4,1}$ is half of the area $A(q)$ defined by $q$ relative to $\mathcal{T}_{3}, A\left(q^{\prime}\right)=A(q) / 2$. We can also consider $q^{\prime}$ as a polygonal closed curve relative to the standard tessellation $\mathcal{T}=\mathcal{T}_{4}$ of $\mathbb{R}^{2}$ into unit squares. Note that $\left|q^{\prime}\right|_{\mathcal{T}_{4}}=\left|q^{\prime}\right|_{\mathcal{T}_{4,1}}=|q|_{\mathcal{T}_{3}}$ and that the property (a) of Corollary 1.10 holds for $q^{\prime}$ with the constant $K=1$ relative to $\mathcal{T}_{4}$. Thus, by proven part (a), the area $A\left(q^{\prime}\right)$ can be computed in space $O\left(\left(\log \left|q^{\prime}\right|_{\mathcal{T}_{4}}\right)^{3}\right)=O\left(\left(\log |q|_{\mathcal{T}_{3}}\right)^{3}\right)=O\left((\log |q|)^{3}\right)$ or in time

$$
O\left(\left|q^{\prime}\right|_{\mathcal{T}_{4}}^{5} \log \left|q^{\prime}\right|_{\mathcal{T}_{4}}\right)=O\left(|q|_{\mathcal{T}_{3}}^{5} \log |q|_{\mathcal{T}_{3}}\right)=O\left(|q|^{5} \log |q|\right)
$$

for the reason that $K=1$ and we can use $n=2$ in (10.17) unless $\left|q^{\prime}\right|_{\mathcal{T}_{4}}=2$ in which case $A\left(q^{\prime}\right)=A(q)=0$. Since $A(q)=2 A\left(q^{\prime}\right)$, our proof is complete.

It is tempting to try to lift the restrictions of Corollary 1.10 to be able to compute, in polylogarithmic space, the area $A(c)$ defined by an arbitrary polygonal closed curve $c$ in the plane equipped with a tessellation $\mathcal{T}$ into unit squares. Approximation approach of Theorem 1.9 together with construction of actual elementary homotopies for the approximating path $\zeta_{M}(c)$ of Theorem 1.8 that seem to indicate the sign of the pieces $A\left(c_{i} \zeta_{M}\left(c_{i}\right)^{-1}\right)$ of the intermediate area between $c$ and $\zeta_{M}(c)$, both done in polylogarithmic space, provide certain credibility to this idea.

However, in the general situation, this idea would not work because the rational number $A(c)$ might have an exponentially large denominator, hence, $A(c)$ could take polynomial space just to store (let alone the computations). An example would be provided by a polygonal closed curve $c=c(n)$, where $n \geq 2$ is an integer, such that 
$|c|_{\mathcal{T}}<(n+1)^{2}$ and the denominator of $A(c)$ is greater than $2^{k_{1} n-1}$, where $k_{1}>0$ is a constant. Below are details of such an example.

Let $n \geq 2$ be an integer and let $p_{1}, \ldots, p_{\ell}$ be all primes not exceeding $n$. Recall that there is a constant $k_{1}>0$ such that $p_{1} \ldots p_{\ell}>2^{k_{1} n}$, see [14. We construct line segments $c_{3 i+1}, c_{3 i+2}, c_{3 i+3}$ for $i=0, \ldots, \ell-1$ and $c_{3 \ell+1}$ of a polygonal closed curve $c=c(n)$ by induction as follows. Let the initial vertex $\left(c_{1}\right)_{-}$of $c_{1}$ be the point (with coordinates) $(0,0),\left(c_{1}\right)_{-}:=(0,0)$. Proceeding by induction on $i \geq 0$, assume that the point $\left(c_{3 i+1}\right)_{-}=\left(x_{0}, y_{0}\right)$ is already defined. Then the line segment $c_{3 i+1}$ goes from the point $\left(c_{3 i+1}\right)_{-}$to the point $\left(x_{0}, y_{0}+1\right)=\left(c_{3 i+1}\right)_{-}+(0,1)$, written $c_{3 i+1}=\left[\left(c_{3 i+1}\right)_{-},\left(c_{3 i+1}\right)_{-}+(0,1)\right]$. Next, we set

$c_{3 i+2}:=\left[\left(c_{3 i+1}\right)_{+},\left(c_{3 i+1}\right)_{+}+(-1,0)\right], \quad c_{3 i+3}:=\left[\left(c_{3 i+2}\right)_{+},\left(c_{3 i+2}\right)_{+}+\left(p_{i+1},-1\right)\right]$

and, completing the induction step, define $\left(c_{3(i+1)+1}\right)_{-}:=\left(c_{3 i+3}\right)_{+}$, see Fig. 10.4, where the case $\ell=3$ with $p_{1}=2, p_{2}=3, p_{3}=5$ is depicted.

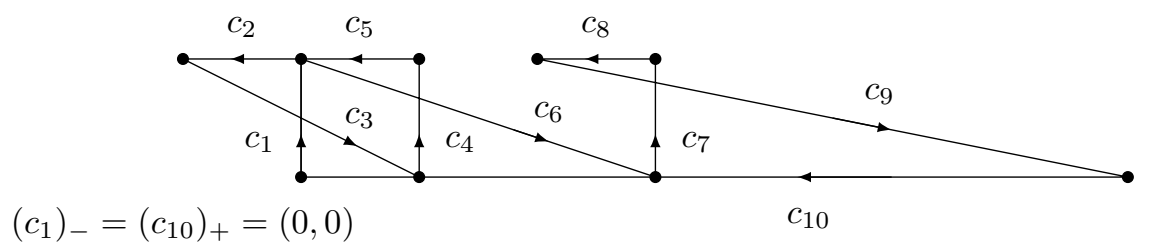

Fig. 10.4

Finally, we define $\left(c_{3 \ell+1}\right)_{-}:=\left[\left(c_{3 \ell}\right)_{+},\left(c_{1}\right)_{-}\right]$and $c=c(n):=c_{1} \ldots c_{3 \ell} c_{3 \ell+1}$, see Fig. 10.4 where $c_{3 \ell+1}=c_{10}$.

It is not difficult to check that

$$
|c|_{\mathcal{T}}=\ell+2 \sum_{i=1}^{\ell} p_{i}<(n+1)^{2}
$$

and that the area $A(c)$ defined by $c$ is the following sum of areas of $2 \ell$ triangles

$$
\begin{aligned}
A(c) & =\sum_{i=1}^{\ell} \frac{1}{2}\left(\frac{1}{p_{i}}+\left(p_{i}-1\right)\left(1-\frac{1}{p_{i}}\right)\right)=\frac{1}{2} \sum_{i=1}^{\ell}\left(\frac{1}{p_{i}}+\left(p_{i}-1\right)-1+\frac{1}{p_{i}}\right)= \\
& =\sum_{i=1}^{\ell} \frac{1}{p_{i}}+\frac{1}{2} \sum_{i=1}^{\ell}\left(p_{i}-2\right)=\frac{\sum_{i=1}^{\ell} \frac{p_{1} \ldots p_{\ell}}{p_{i}}}{p_{1} \ldots p_{\ell}}+\frac{1}{2} \sum_{i=1}^{\ell}\left(p_{i}-2\right) .
\end{aligned}
$$

Since $p_{1} \ldots p_{\ell}>2^{k_{1} n}$, it follows that, after possible cancelation of 2 , the denominator of $A(c)$ is greater than $2^{k_{1} n-1}$.

It would be of interest to study similar problems for tessellation of the hyperbolic plane into regular congruent $2 g$-gons, where $g \geq 2$, which would be technically close to the precise word problem and to the minimal diagram problem for the standard group presentation

$$
\left\langle a_{1}, a_{2} \ldots, a_{2 g-1}, a_{2 g} \| a_{1} a_{2} a_{1}^{-1} a_{2}^{-1} \ldots a_{2 g-1} a_{2 g} a_{2 g-1}^{-1} a_{2 g}^{-1}=1\right\rangle
$$

of the fundamental group of an orientable closed surface of genus $g \geq 2$. Hopefully, there could be developed a version of calculus of brackets for such presentations that would be suitable for these problems. 
It is likely that suitable versions of calculus of brackets could be developed for problems on efficient planar folding of RNA strands which would provide polylogarithmic space algorithms for such problems. Recall that available polynomial time algorithms for such problems, see 27, [28, 29], 40, use polynomial space. This approach would give a chance to do, also in polylogarithmic space, maximization of foldings relative to various parameters similar to those discussed in Theorem 1.5.

Acknowledgements. The author is grateful to Tim Riley for bringing to the author's attention folklore arguments that solve the precise word problem for presentation $\langle a, b \| a=1, b=1\rangle$ in polynomial time, the article [20, and the similarity between the precise word problem for presentation $\langle a, b \| a=1, b=1\rangle$ and the problem on efficient planar folding of RNA strands. The author thanks the referee for a number of useful remarks and suggestions.

\section{REFERENCES}

[1] A. V. Anisimov, The group languages, Kibernetika (Kiev) 4(1971), 18-24.

[2] R. Armoni, A. Ta-Shma, A. Wigderson and S. Zhou, A $(\log n)^{4 / 3}$ space algorithm for $(s, t)$ connectivity in undirected graphs, J. Assoc. Comput. Mach. 47(2000), 294-311.

[3] S. Arora and B. Barak, Computational complexity - a modern approach, Cambridge Univ. Press, 2009.

[4] D. Barrington, P. Kadau, K. Lange and P. McKenzie, On the complexity of some problems on groups input as multiplication tables, J. Comput. System Sci. 63(2001), 186-200.

[5] J.-C. Birget, A. Yu. Ol'shanskii, E. Rips, and M. V. Sapir, Isoperimetric functions of groups and computational complexity of the word problem, Ann. Math. 156(2002), 467-518.

[6] W. W. Boone, On certain simple undecidable problems in group theory, V, VI, Indag. Math. 19(1957), 22-27, 227-232.

[7] W. W. Boone, The word problem, Ann. Math. 70(1959), 207-265.

[8] V. V. Borisov, Simple examples of groups with unsolvable word problems, Mat. Zametki 6(1969), 521-532.

[9] D. Cummins, Dehn functions, the word problem, and the bounded word problem for decidable group presentations, preprint, arXiv:1212.2024 [math.GR].

[10] M. Dehn, Über unendliche diskontinuierliche Gruppen, Math. Ann. 71(1911), 116-144.

[11] R. I. Grigorchuk and P. F. Kurchanov, On the width of elements in free groups, Ukrainian Math. J. 43(1991), 911-918.

[12] R. I. Grigorchuk and S. V. Ivanov, On Dehn functions of infinite presentations of groups, Geom. Funct. Anal. 18(2008), 1841-1874.

[13] M. Gromov, Hyperbolic groups, Essays in Group Theory(S. Gersten, ed.), MSRI Publ. 8, Springer-Verlag, 1987, 75-263.

[14] G. H. Hardy and E. M. Wright, An introductton to the theory of numbers, Oxford Univ. Press, London, 6th ed., 2008.

[15] S. V. Ivanov, The free Burnside groups of sufficiently large exponents, Internat. J. Algebra Comp. 4(1994), 1-308.

[16] R. J. Lipton and Y. Zalcstein, Word problems solvable in logspace, J. Assoc. Comput. Mach. 24(1977), 522-526.

[17] R. J. Lipton, L. Snyder, and Y. Zalcstein, The complexity of word and isomorphism problems for finite groups, Proc. Conf. on Information Sciences and Systems, 1976, pp. 33-35.

[18] R. C. Lyndon and P. E. Schupp, Combinatorial group theory, Springer-Verlag, 1977.

[19] W. Magnus, J. Karras, D. Solitar, Combinatorial group theory, Interscience Publ., 1966.

[20] A. Majumdar, J. M. Robbins, M. Zyskin, Tangent unit-vector fields: nonabelian homotopy invariants and the Dirichlet energy, C. R. Math. Acad. Sci. Paris 347(2009), 1159-1164.

[21] A. Majumdar, J. M. Robbins, M. Zyskin, Tangent unit-vector fields: nonabelian homotopy invariants and the Dirichlet energy, Acta Math. Sci. Ser. B Engl. Ed. 30 30(2010), 13571399.

[22] A. A. Markov, The impossibility of certain algorithms in the theory of associative systems I, Dokl. Akad. Nauk 55(1947), 583-586. 
[23] A. A. Markov, The impossibility of certain algorithms in the theory of associative systems II, Dokl. Akad. Nauk 58(1947), 353-356.

[24] N. Megiddo and U. Vishkin, On finding a minimum dominating set in a tournament, Theoret. Comput. Sci. 61(1988), 307-316.

[25] P. S. Novikov, On algorithmic unsolvability of the problem of identity, Doklady Akad. Nauk SSSR 85(1952), 709-712.

[26] P. S. Novikov, On the algorithmic unsolvability of the word problem in group theory, Trudy Mat. Inst. Steklov 44(1955), 1-143.

[27] R. Nussinov, G. Pieczenik, J. Griggs, and D. Kleitman, Algorithms for loop matchings, SIAM J. Appl. Math. 35(1978), 68-82.

[28] R. Nussinov and A. B. Jacobson, Fast algorithm for predicting the secondary structure of single stranded RNA, Proc. Natl. Acad. Sci. USA 77(1980), 6309-6313.

[29] R. Nussinov, B. Shapiro, and S. Le, J. V. Maizel, Speeding up the dynamic algorithm for planar RNA folding. Math. Biosci. 100(1990), 33-47.

[30] A. Yu. Ol'shanskii, Geometry of defining relations in groups, Nauka, Moscow, 1989; English translation: Math. and Its Applications, Soviet series, vol. 70, Kluwer Acad. Publ., 1991.

[31] A. Yu. Ol'shanskii, On calculation of width in free groups, London Math. Soc. Lecture Note Ser. 204(1995), 255-258.

[32] C. H. Papadimitriou, Computational complexity, Addison-Wesley Publ., 1994.

[33] C. H. Papadimitriou and M. Yannakakis, On limited nondeterminism and the complexity of the $V$-C dimension, J. Comput. System Sci. 53(1996), 161-170.

[34] E. L. Post, Recursive unsolvability of a problem of Thue, J. Symb. Logic 12(1947), 1-11.

[35] T. R. Riley, Private communication.

[36] T. R. Riley, Computing area in presentations of the trivial group, preprint, arXiv:1606.08833 [math.GR].

[37] J. J. Rotman, An introduction to the theory of groups, Springer-Verlag, 1995.

[38] W. J. Savitch, Relationships between nondeterministic and deterministic tape complexities, J. Comput. System Sci. 4(1970), 177-192.

[39] A. M. Turing, The word problem in semigroups with cancellation, Ann. Math. 52(1950), 491-505.

[40] M. Zuker and P. Stiegler, Optimal computer folding of large RNA sequences using thermodynamics and auxiliary information, Nucleic Acids Res. 9(1981), 133-148.

Department of Mathematics, University of Illinois, 1409 West Green Street, UrBANA, IL 61801, USA

E-mail address: ivanov@illinois.edu 